\title{
Human-Robot-Interaction
}

\section{Evaluation, Konzeption und Umsetzung einer Anwendung für den NAO-Roboter der Firma Aldebaran Robotics zur Förderung autistischer Kinder.}

\author{
Bachelorarbeit \\ Zur Erlangung des akademischen Grades \\ Bachelor of Engineering (B. Eng.) \\ An der Technischen Hochschule Wildau
}

\section{Technische Hochschule Wildau}

Fachbereich Ingenieurwesen / Wirtschaftsingenieurwesen

Studiengang Telematik

Eingereicht am:

26.08.2014

Verfasser:

Benjamin Körner

Geb. am:

12.05.1984

Gutachter:

Prof. Dr. Janett Mohnke

Zweitgutachter:

Dipl.-Math. Bernd Weißbach

Themenstellender Betrieb:

Technische Hochschule Wildau 


\section{Bachelorarbeit}

Eingang Antrag

\begin{tabular}{|c|c|c|c|}
\hline & Bache & rarbei & $\begin{array}{c}\text { Eingang Antrag. } \\
\text { EINGEGANGEN } \\
03 . \text { Astil } 2014 \\
\text { FB IWI }\end{array}$ \\
\hline Name, Vorname: & Körner, Benjamin & Matrikelnummer: & 0117410355 \\
\hline Studiengang: & Telematik & Seminargruppe: & T11 \\
\hline Betreuender & Prof Dr lanett Mohnke & Beginn der Arbeit: & 14.04 .2014 \\
\hline Hochschuldozent: & & Abgabetermin: & 01.08 .2014 \\
\hline Zweitgutachter: & Dipl. Math. Bernd Weissbach & Betriebl. Betreuer: & Prof. Dr. Janett Mohnke \\
\hline Themensteller: & Technische Hochschule Wildau & Straße, PLZ, Ort: & $\begin{array}{l}\text { Hochschulring } 1 \\
15745 \text { Wildau }\end{array}$ \\
\hline
\end{tabular}

Kurzthema: Human-Robot-Interaction: Evaluation, Konzeption und Umsetzung einer Anwendung für den NAO-Roboter der Firma Aldebaran Robotics zur Förderung autistischer Kinder.

\section{Kurzthema}

englisch:

\begin{abstract}
Human-Robot-Interaction: evaluation, conception and implementation of an application for the NAO robot developed by Aldebaran Robotics to support autistic children.
\end{abstract}

Zielstellung:

Es soll eine prototypische Anwendung für den NAO Roboter entstehen, die therapeutisch unterstützend bei der Förderung der sozialen Interaktion autistischer Kinder eingesetzt werden kann.

Inhaltliche Anforderungen / Teilaufgaben:

Konsultationen: nach Vereinbarung mit dem betreuenden Hochschullehrer

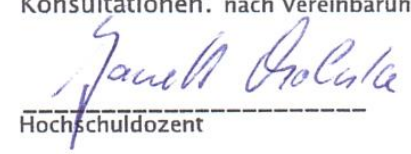

Eingang Abschlussarbeit: (Eingangsstempel)

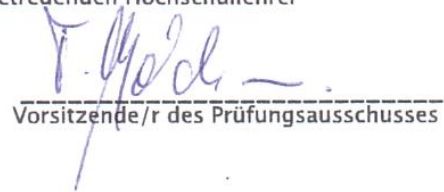

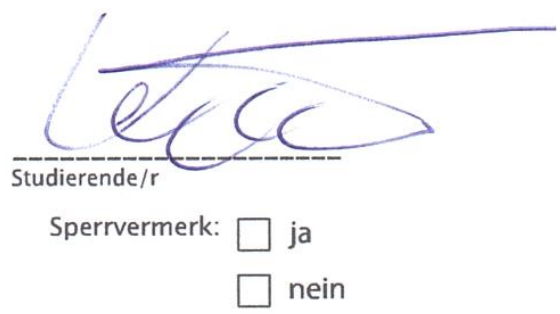




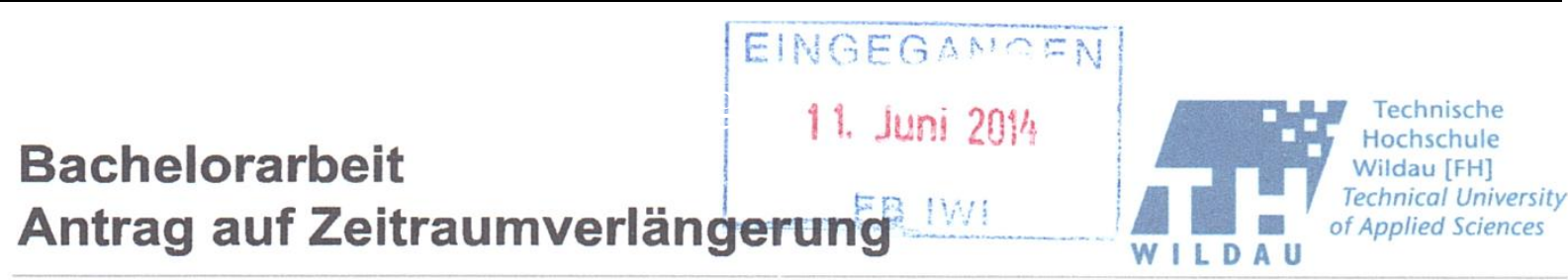

$\begin{array}{llll}\text { Name, Vorname: } & \text { Koerner, Benjamin } & \text { Datum: } & 04.06 .2014 \\ \text { Anschrift: } & \text { Wattstraße 10,12459 Berlin } & \\ \text { Studienrichtung: } & \text { Telematik } & \text { Matr.-Nr.: } & 0117410355 \\ \text { Telefon: } & 0172 / 1037642 & \text { Abgabetermin: } & 01.08 .2014 \\ & & \end{array}$

An den

Vorsitzenden des Prüfungsausschusses

des Fachbereiches Ingenieurwesen/Wirtschaftsingenieurwesen

Bahnhofsstraße 1, 15745 Wildau

Betreff: Antrag auf Verlängerung des Bearbeitungszeitraumes meiner Bachelorarbeit

\section{Begründung:}

Im bisherigen Verlauf der Bachelorarbeit hat sich eine Zusammenarbeit mit einem Therapiezentrum für autistische Kinder ergeben. Diese Zusammenarbeit ermöglicht es mir wichtige Lösungen in meiner BA zu entwickeln. Um erfolgreich mehrere Tests durchzuführen und abzuschließen, bitte ich um eine Zeitraumverlängerung von vier Wochen.

\section{Stellungnahme des/der 1. Betreuers/in:}

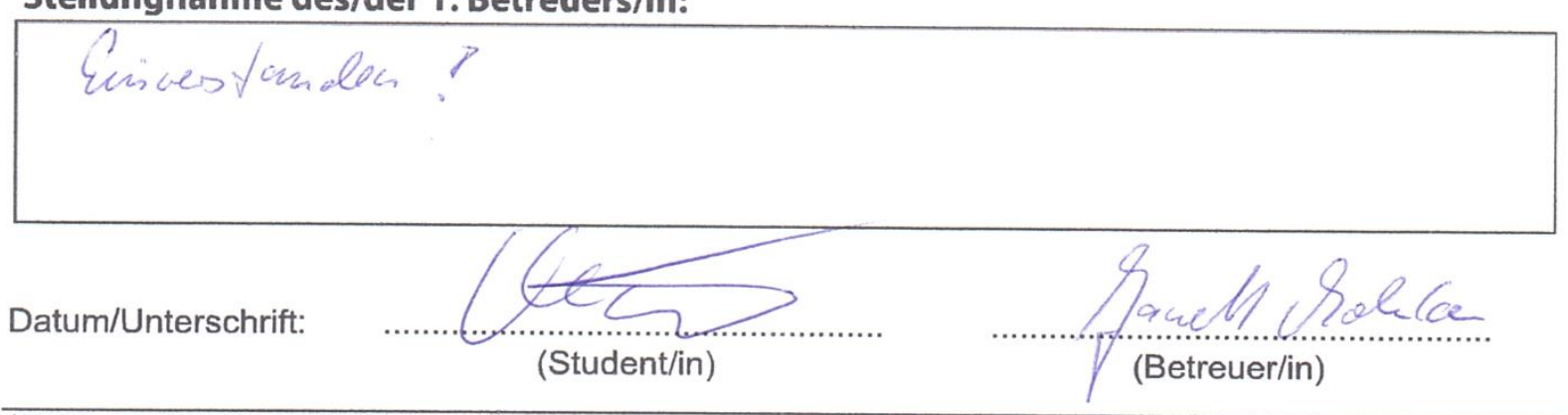

(Nicht vom Studenten auszufüllen)

\section{Entscheidung des Prüfungsausschusses}

ja / mein Verlängerungszeitraum

Datum

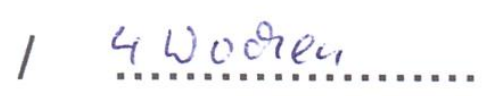

13. Juni 2015...

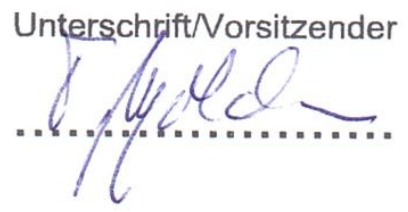

Neuer Abgabetermin:

$29,0 x=2014$ 


\section{Bibliographische Beschreibung}

Körner, Benjamin

Human-Robot-Interaction: Evaluation, Konzeption und Umsetzung einer Anwendung für den NAO-Roboter der Firma Aldebaran Robotics zur Förderung autistischer Kinder.

Bachelorarbeit, Technische Hochschule Wildau [FH] 2014, 91 Seiten, 75 Abbildungen, 17 Tabellen, 55 Quellenangaben, 8 Anlagen, 1 Beilage

\section{Ziel}

Entwicklung einer prototypischen Anwendung für den NAO-Roboter der Firma Aldebaran Robotics, die therapeutisch unterstützend bei der Förderung der sozialen Interaktion mit autistischen Kindern eingesetzt werden kann.

\section{Inhalt}

Aufzeigen der Grundlagen zum Thema Autismus und Human-Robot-Interaktion mit Fokus auf den NAO-Roboter

Evaluation und Auswahl einer geeigneten Entwicklungsumgebung für die Entwicklung einer Anwendung zur Interaktion mit dem NAO

Prüfen der Integrationsfähigkeit des Leap Motion Controllers in die Anwendung

Konzeption einer Anwendung zur HRI mit autistischen Kindern

Umsetzung des Konzepts

Anwender- und Entwicklerdokumentation

\section{Stichworte:}

NAO, Roboter, Human-Robot-Interaction, Autismus, Socially Assistive Robotics 


\section{Selbstständigkeitserklärung}

Ich bestätige durch meine Unterschrift, dass ich die Voraussetzungen zur Anfertigung der Abschlussarbeit gemäß der für mich gültigen Studien- und

Prüfungsordnung erfülle.

Wildau, 26.08.2014

Benjamin Körner 


\section{Hinweise zum Lesen der Arbeit}

Die in dieser Arbeit verwendeten Glossareinträge werden mit einem * am Ende des Wortes gekennzeichnet. Das Glossar befindet sich am Ende des Dokumentes. Des Weiteren steht dem Leser ein Abkürzungsverzeichnis zur Verfügung, welches ebenfalls in der Gliederung nach dem Hauptteil zu finden ist. Erklärungsbedürftige Einträge im Abkürzungsverzeichnis werden im Glossar näher beschrieben. Sofern ein Begriff nicht im Glossar oder im Abkürzungsverzeichnis zu finden ist, wird dieser in einer Fußnote auf derselben Seite, auf welcher der Begriff erwähnt wurde, ausreichend erläutert. Die Quellenangaben im Text werden nach dem ACM Standard [Nummer] gesetzt, um den Textfluss zu erhalten. Programmtechnische Methoden, Funktionen und Bibliotheken werden in der Schriftart Curier New formatiert dargestellt. Unter Abbildungen, Tabellen und Auflistungen ist stets die Quelle mit angegeben. Beschriftungen, die keine Quellenangabe enthalten, sind eigenhändig vom Autor erstellt worden. 


\section{Danksagung}

Zunächst möchte ich mich an dieser Stelle bei denjenigen bedanken, die mich während der Anfertigung dieser Bachelor-Arbeit unterstützt und motiviert haben.

Ein besonderer Dank geht an meine Betreuerin, Frau Prof. Dr. Janet Mohnke, die mir stets unterstützend zur Seite stand. Durch ihre Anregungen, ihr kreatives Hinterfragen und ihren Kontakt zur Autismus-Expertin Kerstin Michlo brachte sie mich besonders in der Startphase auf einen guten Weg.

Frau Michlo möchte ich danken, dass sie mir einen interessanten und tiefgreifenden Einstieg in das Thema Autismus ermöglichte und den Kontakt zur Ganztags-Kleinklasse I der Frühfördereinrichtung für autistische Kinder in Berlin Friedrichshain vermittelte.

Allen Betreuern der Ganztags-Kleinklasse I möchte ich herzlichst danken, dass sie mir geduldig meine Fragen beantworteten und die Räumlichkeiten für die Testreihen mit dem Roboter und dem autistischen Kind Theo ${ }^{1}$ zur Verfügung stellten. Ein besonderer Dank gilt dabei Frau Grohmann, die als Bezugsbetreuerin von Theo umfangreiches therapeutisches Wissen zur Verfügung stellte.

Ein großer Dank geht an Stefan Paffrath, der viel Zeit und Mühe in die Rechtschreibkorrektur dieser Arbeit investierte.

Herzlichst möchte ich meiner Frau Felicitas danken, welche mir uneingeschränkt den Rücken im Familienleben freigehalten und mich immer wieder motiviert hat. Ich danke ihr ebenfalls für ihr geduldiges Korrekturlesen.

Mein ganz besonderer Dank gilt abschließend meiner Familie, die mir mit ihrem geduldigen Verständnis in der Bachelorphase beistand.

\footnotetext{
${ }^{1}$ Der Name wurde aus datenschutztechnischen Gründen vom Autor geändert
} 


\section{Inhaltsverzeichnis}

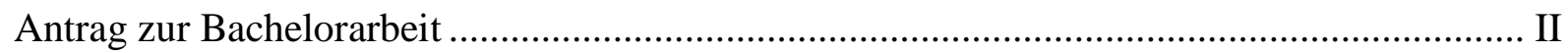

Antrag auf Zeitraumverlängerung ................................................................................... III

Bibliographische Beschreibung ................................................................................. IV

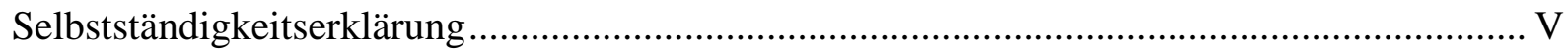

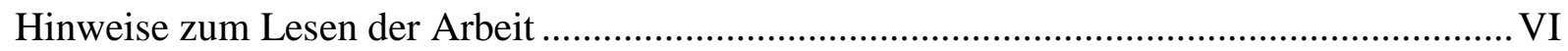

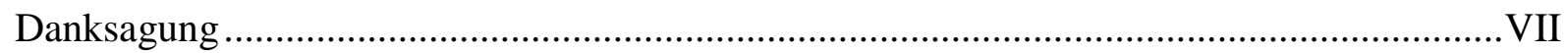

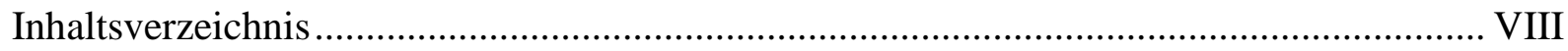

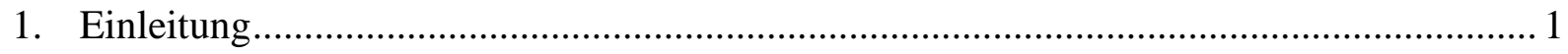

2. Theoretische Annäherung an das Thema ,Autismus“ “................................................. 4

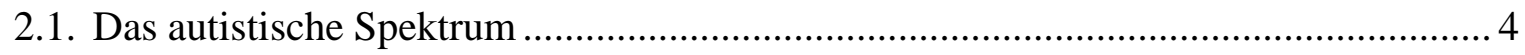

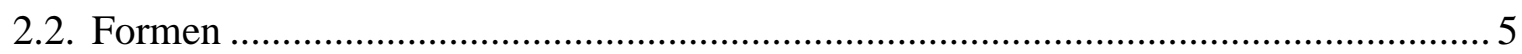

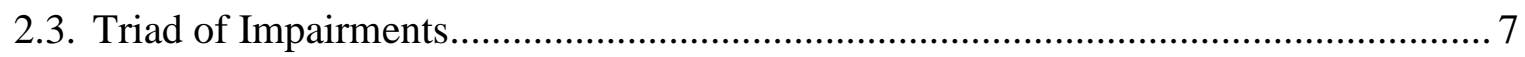

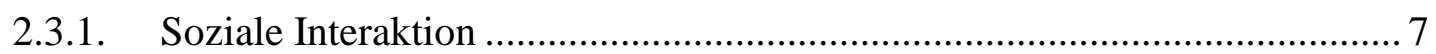

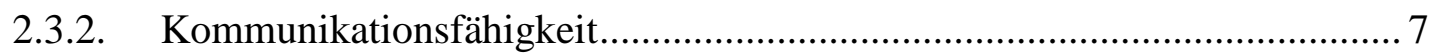

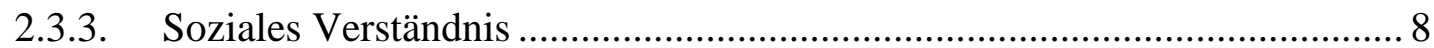

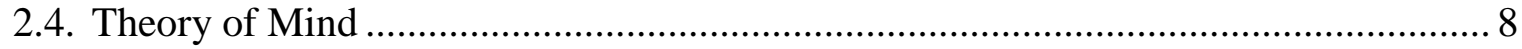

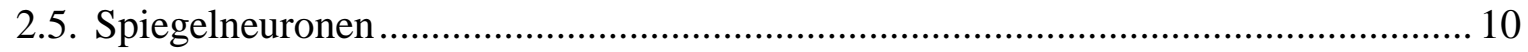

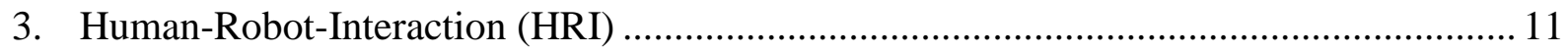

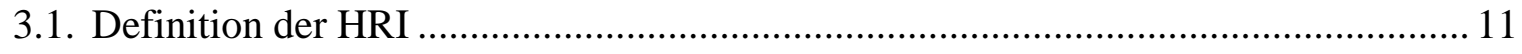

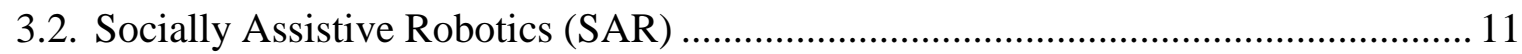

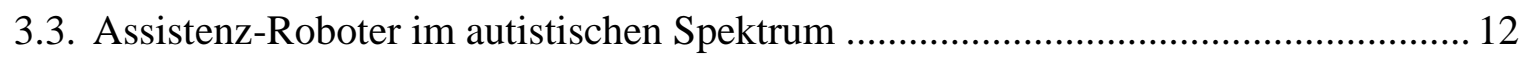

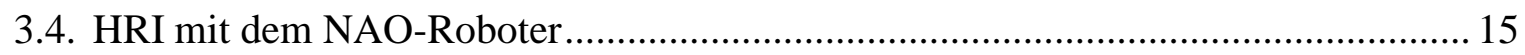

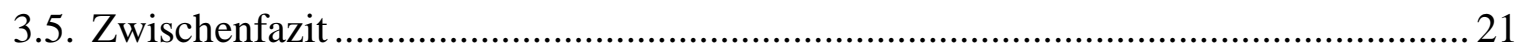

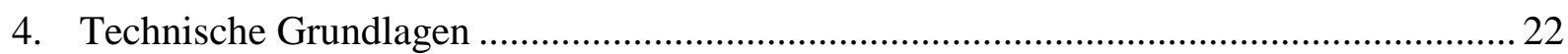

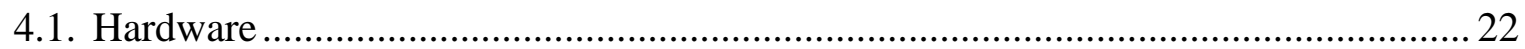

4.1.1. Taktile und mechanische Sensoren ....................................................... 23

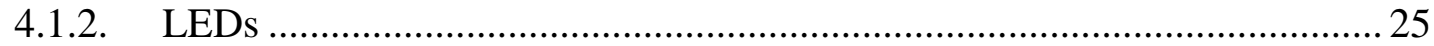

Benjamin Körner $\quad$ VIII 


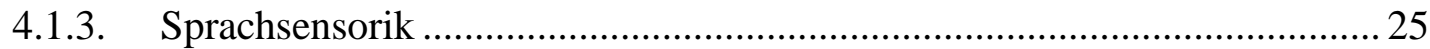

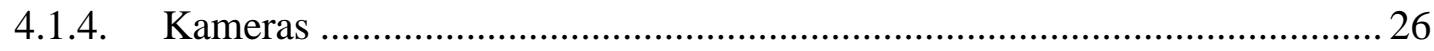

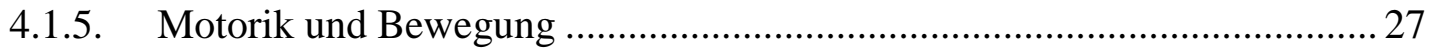

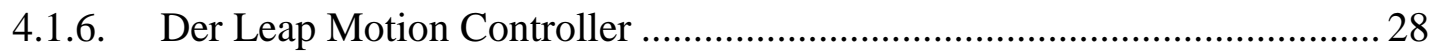

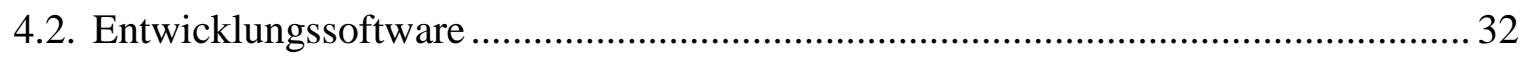

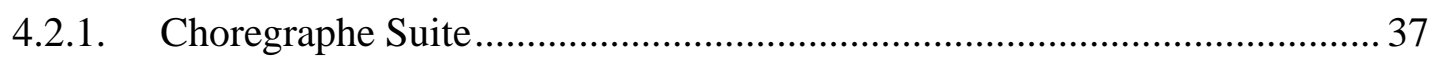

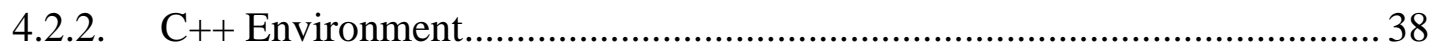

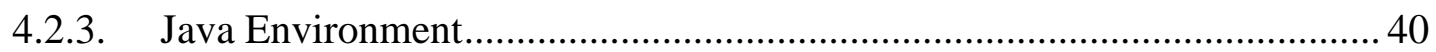

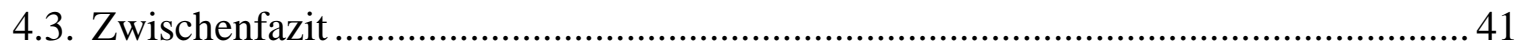

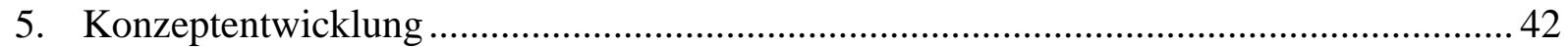

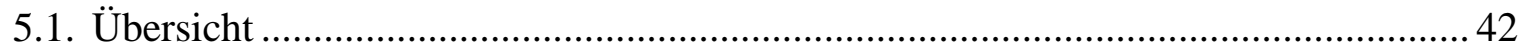

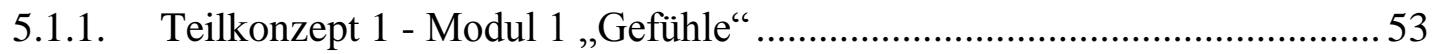

5.1.2. Teilkonzept 2 - Modul 2 „Berühre mich““................................................. 60

5.1.3. Teilkonzept 3 - Modul 3 „Geschichte“ ...................................................... 64

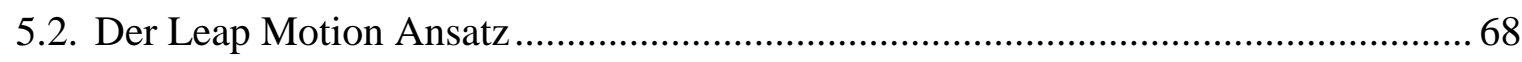

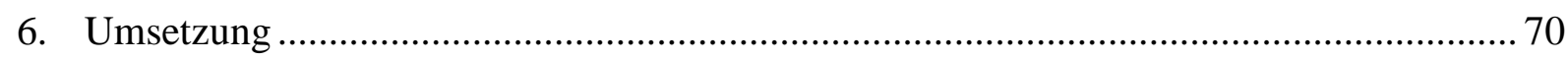

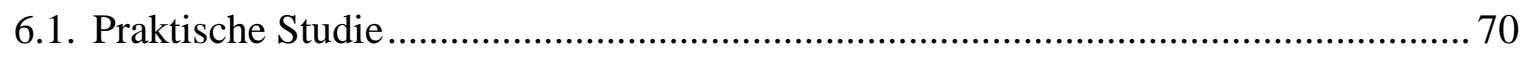

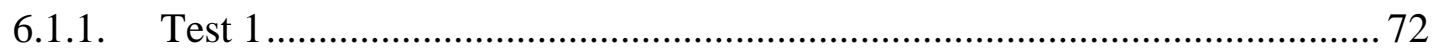

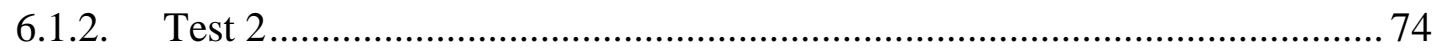

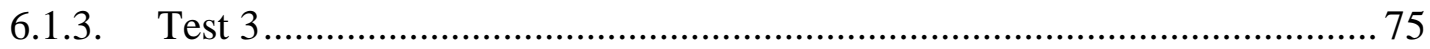

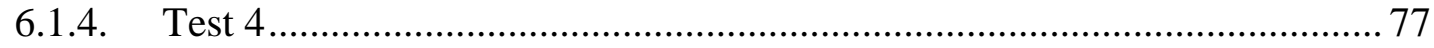

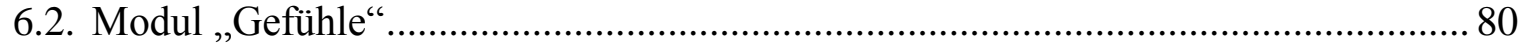

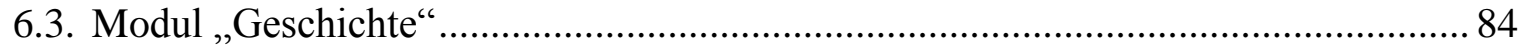

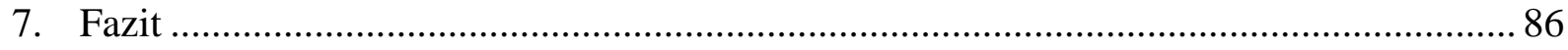

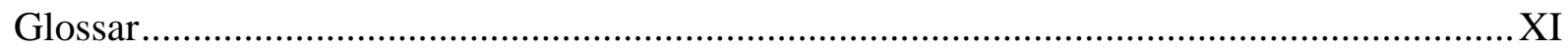

Abkürzungsverzeichnis ............................................................................................ XIII

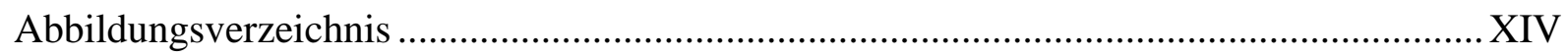

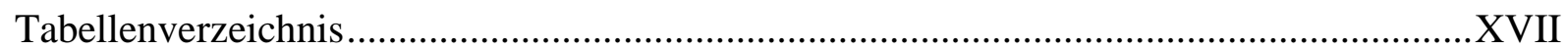

Benjamin Körner $\quad$ IX 


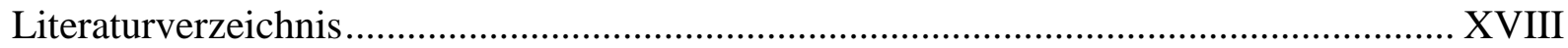

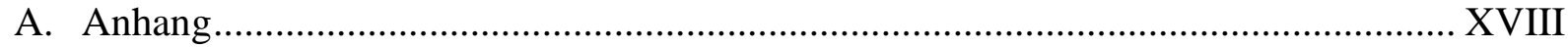

A.1. Erstellung von Motions mittels des Animationsmodus und Export in C++ ........ XVIII

A.2. Programmablaufplan Modul „Moderator“، ...........................................................XXII

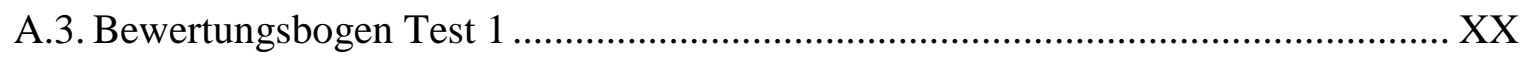

A.4. Bewertungsbogen Test 2 .............................................................................XXI

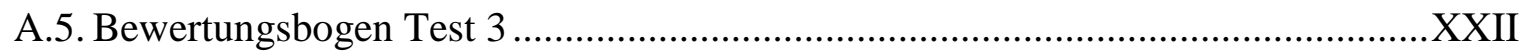

A.6. Bewertungsbogen Test 4 _........................................................................... XXIII

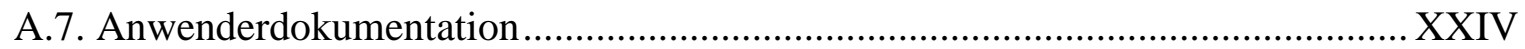

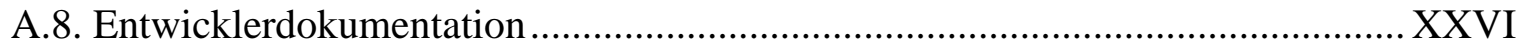

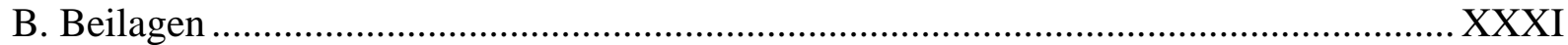

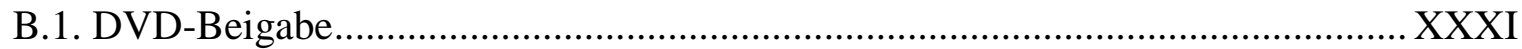




\section{Einleitung}

Das Kapitel „Einleitung“ soll den Leser thematisch auf den Inhalt dieser Arbeit einstimmen. Es wird zum einen die Motivation zur vorliegenden Projektidee erläutert und zum anderen eine Abgrenzung zum Thema gesetzt. Des Weiteren werden die grundlegende Zielstellung und der strukturelle Aufbau dieser Arbeit erörtert.

Menschen - Roboter - Autismus - Soziale Interaktion. Was haben diese Begriffe gemeinsam? Sie finden sich alle in der ingenieurswissenschaftlichen Thematik der Human-RobotInteraction (HRI) wieder. Diese Mensch-Maschine-Interaktion ist längst keine Zukunftsversion mehr, wie man sie aus Science-Fiction-Filmen kennt. Dass Menschen und Roboter miteinander kommunizieren, ist realer denn je. An der Technischen Hochschule in Wildau wird seit diesem Jahr das konsekutive Studienfach „Embedded C++“ angeboten. In diesem Fach haben Studenten die Möglichkeit, mit dem humanoiden Roboter NAO der französischen Firma Aldebaran Robotics Bekanntschaft zu machen. Derzeit forschen und arbeiten über 60 Nationen weltweit in Projekten und an Studien auf verschiedenen Themengebieten mit diesem NAO-Roboter. Unter anderem wird er bei der Fußball- WM der Roboter, dem RoboCup [1], in der medizinischen Betreuung von Kindern [2] und im HomeAssistance-Bereich eingesetzt [3]. Auch im therapeutischen Einsatz für autistische Kinder findet er weltweit großes Interesse [4-7]. Dieses Interesse teilen auch die Professorin der TH Wildau,

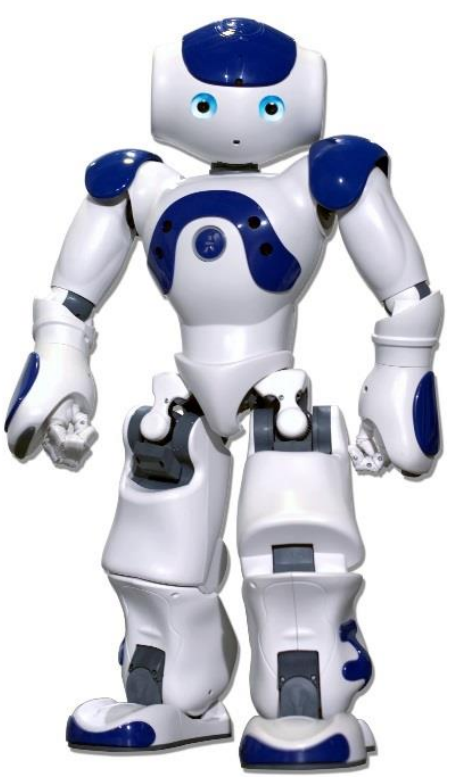

Abbildung 1 - Der humanoide NAORoboter Quelle: Aldebaran Robotics

Frau Prof. Dr. Janett Mohnke, sowie der Autor dieser Arbeit. Bedingt durch den eben genannten Umstand und die Heranführung an den NAO-Roboter im Studienfach „Embedded $\mathrm{C}++{ }^{*}$ entwickelte sich die Idee eines Forschungsthemas. Durch Gespräche mit der Ambulanzlehrerin für Autismus, Kerstin Michlo, welche für den Autismus Deutschland Landesverband Berlin e.V. ${ }^{2}$ tätig ist, ergab sich eine Möglichkeit der Zusammenarbeit im Raum Berlin. Die Ergebnisse dieser Zusammenarbeit werden im Kapitel 6.1 „Praktische Studie“ eingehend erläutert.

Aktuelle Studien belegen, dass derzeit ca. 1\% der Bevölkerung an Autismus erkrankt. Mit anderen Worten heißt das, dass eines von 88 Kindern autistische Symptome aufweist [8].

\footnotetext{
${ }^{2}$ Link zur Website: http://www.autismus-berlin.de/ Zugriff: 31.07.2014
} 
Diese Kinder bedürfen einer besonderen therapeutischen Zuwendung und Aufmerksamkeit, damit sie die grundlegenden gesellschaftlichen Umgangsformen lernen und so mit ihrer Umwelt kommunizieren können.

Wie passen Autismus und der NAO-Roboter zusammen?

Die Kommunikation mit anderen ist für die meisten Menschen eine natürliche Handlung im Alltag. Dabei benutzen sie unbewusst mehr Körperbewegungen und Mimik im Gesicht, als bewusst wahrgenommen wird. Autisten fällt diese Kommunikation meist sehr schwer, da sie die vielen Gesten und mimischen Bewegungen oft nicht verstehen oder als störend empfinden [9]. Sie können diese nicht filtern und ziehen sich zurück. Mit dem humanoiden NAORoboter, der sehr menschlich wirkt, ist es möglich, die störenden Faktoren auszuschalten und ihn nur auf einfachste Handlungen zu beschränken. Ebenfalls motivierend für das Thema ist die Aussage von Anjana Bhat von der University of Connecticut. Sie erklärt, dass ,Autistische Kinder ... sich zu humanoiden Robotern hingezogen [fühlen, da] sie [...] weniger kompliziert [sind] als Menschen." [10] Weitere wissenschaftliche Forschungen ergaben, dass das Konzentrationsvermögen autistischer Kinder stark erhöht ist, sobald sie mit dem NAO interagieren, wie folgendes Zitat von. H. Yussof belegt:

"Interacting with Humanoid Robot NAO generates more concentration level to the Autism Children and they are more focus, when they are exposed to the modules in the Robotbased Intervention Program. “ [9]

Dieser Ansatz und der vielfältige Funktionsumfang des NAO-Roboters sind vielversprechende Grundlagen, eine Anwendung im Bereich der HRI mit autistischen Kindern zu konzeptionieren. Durch die Kommunikations- und Interaktionsfähigkeit des Roboters könnte der Anreiz für Autisten, sich anderen Menschen gegenüber zu öffnen und anzunähern, erhöht werden. Denkbar ist auch, dass sich eine starke Bindung zwischen dem Kind und dem Roboter entwickeln könnte und somit die sozialen Fähigkeiten gefördert, und gefestigt werden. Eine solche Untersuchung wäre somit der wissenschaftlichen sowie der therapeutischen Arbeit mit Autisten und der immer wichtiger werdenden Frage nach der Stellung humanoider Roboter im Gesundheitswesen dienlich.

In dieser Arbeit soll untersucht werden, ob mit dem NAO-Roboter der Firma Aldebaran Robotics eine gezielte Förderung der sozialen Kommunikations- und Interaktionsfähigkeit autistischer Kinder möglich ist. Dazu ist eine ausführliche Betrachtung der Grenzen sozialer Interaktion und Kommunikation von autistischen Kindern notwendig. Weiterhin soll eine 
Anwendung entwickelt werden, die eine Interaktion mit dem NAO-Roboter möglich macht (Sprache, Berührung, Blickkontakt). Dazu müssen die verschiedenen programmtechnischen Entwicklungsmöglichkeiten des NAO-Roboters zuerst evaluiert werden. Die oben genannte Anwendung wird als Teil eines Gesamtkonzeptes implementiert. Auch die Ausarbeitung des Gesamtkonzeptes ist ebenfalls Gegenstand dieser Arbeit.

Der Hauptteil dieser Arbeit lässt sich in drei Bereiche unterteilen. Im ersten Teil werden die thematischen Grundlagen speziell zum autistischen Spektrum und der Human-RobotInteraction erläutert. Im zweiten Teil werden die in Hardware und Software unterteilten technischen Grundlagen des NAO-Roboters beschrieben. Zusätzlich wird im zweiten Teil der Leap Motion Controller näher beleuchtet. Der dritte Teil beinhaltet die Konzeptentwicklung, basierend auf den vorangegangenen Kapiteln, und eine prototypische Umsetzung der Anwendung, welche aus mehreren Modulen bestehen wird. Im Unterabschnitt „Praktische Studie" wird eine Testreihe mit einem autistischen Kind und dem Roboter ausführlich beschrieben und ausgewertet. Das dabei entstandene Modul ist als eine Teilumsetzung des Konzepts zu verstehen.

Um den Rahmen dieser Arbeit nicht zu sprengen und mit dem Wissen, dass der Autor kein ausgebildeter Therapeut für autistische Kinder ist, wird im folgenden Abschnitt das Thema weitestgehend abgegrenzt.

\section{Abgrenzung}

Diese Arbeit ist ausgerichtet auf die Förderung von Kindern mit atypischem Autismus ${ }^{3}$. Die Zielgruppe sind Kinder der 1.-3. Klasse im Alter von sechs bis zehn Jahren. In Zusammenarbeit mit der Grundschule am Friedrichshain in Berlin und der Berliner Autismus-Expertin Kerstin Michlo wird die Anwendung speziell auf ein ausgewähltes Kind hin konzipiert [11]. Dennoch wird das Konzept modular aufgebaut, um auf weiterführende Therapieschwerpunkte eingehen zu können.

Weitere Ausprägungen des autistischen Spektrums, wie das Asperger-Syndrom und der frühkindliche Autismus, werden der Vollständigkeit halber in Kapitel 2.2 kurz ergänzend erläutert. Sie dienen nicht als Grundlage für das in Kapitel 5 entwickelte Konzept.

\footnotetext{
${ }^{3}$ eine ausführliche Beschreibung für den atypischen Autismus ist in Kapitel 2.2 zu finden 


\section{Theoretische Annäherung an das Thema „Autismus“}

Im folgenden Kapitel wird das autistische Spektrum im Allgemeinen beschrieben und auf die Form des atypischen Autismus eingegangen. Dabei werden die nötigen Grundlagen thematisiert, die notwendig sind, um die therapeutischen Ansätze im Konzept zu verstehen. Der Schwerpunkt liegt dabei auf dem atypischen Autismus. Des Weiteren wird gezielt auf die Theory of Mind und die neuesten Erkenntnisse in der Erforschung der Spiegelneuronen eingegangen. Diese Erkenntnisse werden ebenfalls Schwerpunkte für die spätere Konzepterstellung bilden.

\subsection{Das autistische Spektrum}

Autismus ist eine tiefgreifende Entwicklungsstörung, welche hirnorganisch bedingt ist. Die Ursachen sind neurologischer, genetischer oder neurochemischer Natur. Sie führen zu einer Störung des Zentralnervensystems, die mit einer tiefgreifenden Störung der Informations- und Wahrnehmungsverarbeitung einhergehen. Ein Zitat des Mediziners und Erstbeschreibers des frühkindlichen Autismus, Leo Kanner ${ }^{4}$, fasst die Eigenschaften des autistischen Spektrums sehr gut zusammen.

\section{„Die herausragende fundamentale pathognomonische ${ }^{5}$ Störung ist die von Geburt an bestehende Unfähigkeit, sich in normaler Weise mit Personen oder Situationen in Beziehung zu setzen. [...] “6}

Darunter ist zu verstehen, dass Menschen mit einer autistischen Störung unfähig sind, sich in andere Menschen hineinzuversetzen. Sie können Gefühle nicht einfach abrufen oder zuordnen. Autisten fühlen sich wohl, wenn sie sich zurückziehen können und nicht mit Menschen interagieren müssen. Diese Interaktion überfordert sie oft, da sie die Mimik und Gestik nicht deuten können. Diese sind ihnen zu komplex. Sie sind also anders ausgedrückt „unfähig, das normale Maß an sozialem Gespür aufzubringen. ${ }^{\text {"6 }}$ Wie das wissenschaftlich nach heutigem Kenntnisstand zu erklären ist, wird näher in Kapitel 2.4 erläutert. Die oben genannten Eigenschaften gelten für alle drei Formen des Autismus. Diese werden im Folgenden erläutert.

\footnotetext{
${ }^{4}$ Leo Kanner +1981 , Mediziner am John-Hopkins-Hospital in Baltimore, Erstbeschreiber des frühkindlichen Autismus und Autor des Buches „Autistische Störungen des affektiven Kontakts“ von 1943

${ }^{5}$ pathognomonisch: für eine Krankheit, ein Krankheitsbild charakteristisch, kennzeichnend

${ }^{6}$ Leo Kanner, ,Autistische Störungen des affektiven Kontakts“ zit. nach [12], Seite 9
} 


\subsection{Formen}

Die drei relevanten Formen des Autismus sind in der Klassifikation des ICD-10-GM ${ }^{7}$ mit folgenden Einträgen wortwörtlich aufgeführt:

\section{ICD10 F84.0 - Frühkindlicher Autismus}

„Diese Form der tief greifenden Entwicklungsstörung ist durch eine abnorme oder beeinträchtigte Entwicklung definiert, die sich vor dem dritten Lebensjahr manifestiert. Sie ist außerdem gekennzeichnet durch ein charakteristisches Muster abnormer Funktionen in den folgenden psychopathologischen Bereichen: in der sozialen Interaktion, der Kommunikation und im eingeschränkten stereotyp repetitiven Verhalten. Neben diesen spezifischen diagnostischen Merkmalen zeigt sich häufig eine Vielzahl unspezifischer Probleme, wie Phobien, Schlaf- und Essstörungen, Wutausbrüche und (autodestruktive ${ }^{8}$ ) Aggression. “

\section{ICD 10 F84.1 - Atypischer Autismus}

„Diese Form der tief greifenden Entwicklungsstörung unterscheidet sich vom frühkindlichen Autismus entweder durch das Alter bei Krankheitsbeginn oder dadurch, dass die diagnostischen Kriterien nicht in allen genannten Bereichen erfüllt werden. Diese Subkategorie sollte immer dann verwendet werden, wenn die abnorme oder beeinträchtigte Entwicklung erst nach dem dritten Lebensjahr manifest wird und wenn nicht in allen für die Diagnose Autismus geforderten psychopathologischen Bereichen (nämlich wechselseitige soziale Interaktionen, Kommunikation und eingeschränktes, stereotyp repetitives Verhalten) Auffälligkeiten nachweisbar sind, auch wenn charakteristische Abweichungen auf anderen Gebieten vorliegen. Atypischer Autismus tritt sehr häufig bei schwer retardierten bzw. unter einer schweren rezeptiven Störung der Sprachentwicklung leidenden Patienten auf."

ICD 10 F84.5 - Asperger-Syndrom

„Diese Störung von unsicherer nosologischer ${ }^{9}$ Validität ist durch dieselbe Form qualitativer Abweichungen der wechselseitigen sozialen Interaktionen, wie für den Autismus typisch, charakterisiert, zusammen mit einem eingeschränkten, stereotypen, sich

\footnotetext{
${ }^{7}$ ICD 10 GM : International Statistical Classification of Diseases and Related Health Problems GM steht für German Modification

${ }^{8}$ selbstzerstörerisch [auto- $=$ selbst-; destruktiv = zersetzend, zerstörend.]

${ }^{9}$ Nosologie - Lehre von den Krankheiten; systematische Beschreibung und Einordnung der Krankheiten 
wiederholenden Repertoire von Interessen und Aktivitäten. Die Störung unterscheidet sich vom Autismus in erster Linie durch fehlende allgemeine Entwicklungsverzögerung bzw. den fehlenden Entwicklungsrückstand der Sprache und der kognitiven Entwicklung. Die Störung geht häufig mit einer auffallenden Ungeschicklichkeit einher. Die Abweichungen tendieren stark dazu, bis in das Jugend- und das Erwachsenenalter zu persistieren. Gelegentlich treten psychotische Episoden im frühen Erwachsenenleben auf. “

Aufgrund der technischen Ausrichtung der Arbeit wird auf eine weitere medizinische Beschreibung der Grundlagen verzichtet. Die obigen drei Auszüge des ICD 10 dienen als Basis, um in den folgenden Kapiteln den Bezug auf die relevanten sozialen Verhaltensweisen herzustellen.

Im Kapitel 2.3 werden die drei typischen Auffälligkeiten in der „sozialen Interaktion“, der „Kommunikation“ und dem „stereotyp repetitiven Verhalten“ nochmals aufgegriffen und erläutert. Sie werden in therapeutischem Umfeld größtenteils unter dem Begriff „Triad of Impairments $^{\text {“ }}$ zusammengefasst. 


\subsection{Triad of Impairments}

Grundlegend zeigt die „Triade der Beeinträchtigungen“ auf einfache und übersichtliche Art, welche drei Hauptbeeinträchtigungen sich durch das gesamte autistische Spektrum ziehen. Sie zeigt, in welchen Bereichen Menschen aus dem autistischen Spektrum Störungen aufweisen.

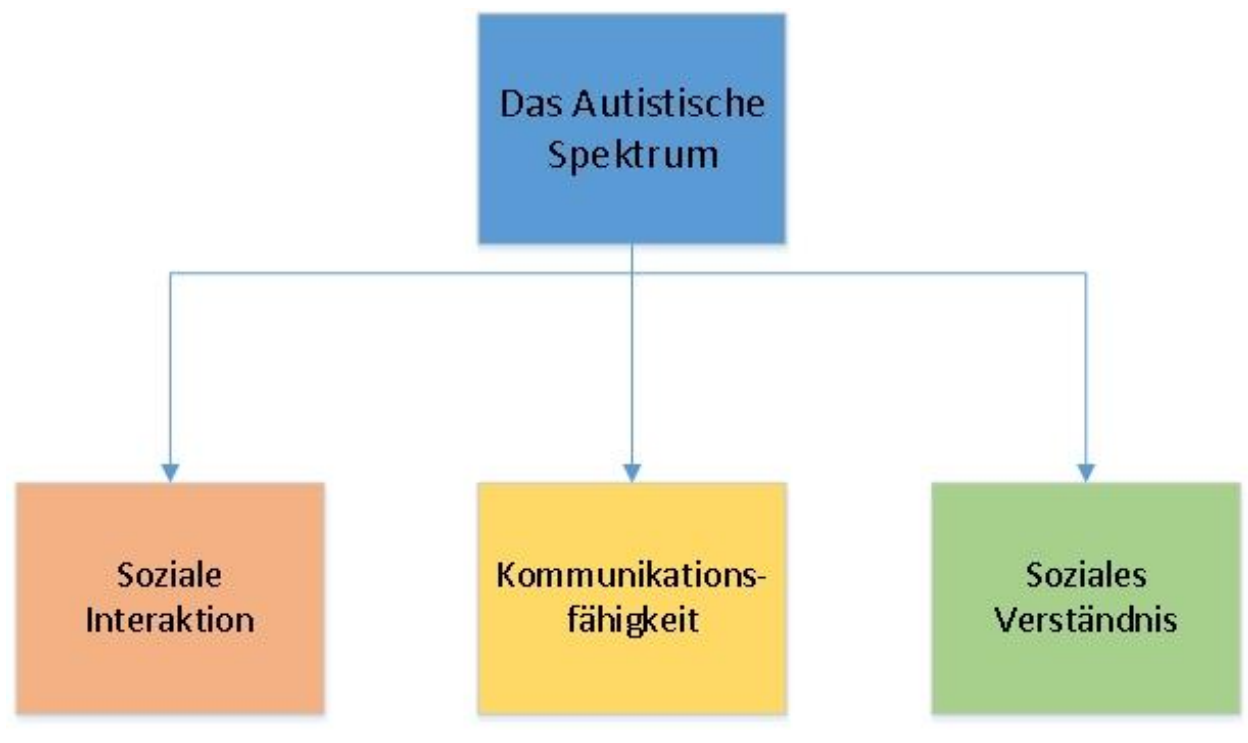

Abbildung 2 - Triad of Impairments

Nachfolgend werden alle drei Beeinträchtigungen erläutert.

\subsubsection{Soziale Interaktion}

Einschränkungen in der sozialen Interaktion werden mit der Unfähigkeit beschrieben, sich situationsbedingt richtig verhalten zu können. Autisten wissen nicht, wie man mit anderen Menschen interagiert. Auffälligkeiten sind beispielweise:

- unangebrachtes Berühren eines anderen Menschen

- Schwierigkeiten den Augenkontakt zu halten

- unangemessene Wahl des Abstandes zu Personen

- fehlendes Verständnis von nonverbalen Äußerungen eines anderen

\subsubsection{Kommunikationsfähigkeit}

Die eingeschränkte Kommunikationsfähigkeit beschreibt die Unfähigkeit, sich vernünftig und effizient mit anderen Menschen zu unterhalten. Beispiele hierfür sind unter anderem:

- Immer wiederholende Fragen

- können nicht „Zwischen den Zeilen“ lesen (wortwörtliches Verstehen)

- reden nur über eigene Interessen 
- wissen nicht, wie sie ihre Hände zur Kommunikation einsetzen sollen

\subsubsection{Soziales Verständnis}

Hierunter versteht man die Unfähigkeit, flexibel zu denken oder anderer Menschen Verhalten bzw. deren Meinung zu verstehen. Weiterhin zählt hierzu das stereotypisch repetitive Verhalten. Hiermit gemeint ist eine monotone, immer wiederkehrende Bewegung, wie z.B. das Wippen mit dem Oberkörper, gemeint. Weitere Aspekte sind:

- fehlendes Verständnis für den Unterschied zwischen Freund, Lehrer oder unbekannter Person

- Unsicherheit, wenn sich eine Situation spontan ändert

- Unfähigkeit, Informationen zu generalisieren

Um auch die mentalen Verhaltensauffälligkeiten zu betrachten, ist es dienlich, die Theory of Mind zu verstehen.

\subsection{Theory of Mind}

Die Theory of Mind (ToM) ist ein wissenschaftlicher Begriff, wenn es um soziale Signale der verbalen und nonverbalen zwischenmenschlichen Interaktion und deren mentales Verständnis geht. Die ToM beschreibt das Verständnis von Begriffen und Gesten mit sozial-emotionalen Gehalt. Das heißt, ein Mensch hat die Fähigkeit, eine Annahme über Bewusstseinsvorgänge in anderen Personen vorzunehmen und diese in der eigenen Person zu erkennen, also Gefühle, Bedürfnisse, Ideen, Absichten, Erwartungen und Meinungen zu vermuten [13]. Ein Beispiel für solch ein soziales Signal ist die Reaktion eines einjährigen Kindes auf das Lächeln seiner Mutter. Bereits in dieser Phase beginnt sich langsam das Empathie-Empfinden auszubilden. Dies ist ein wichtiger Schritt in der Theory of Mind. Für das weitere Verständnis wird speziell das soziale Signal betrachtet.

Das soziale Signal

Das soziale Signal setzt sich aus mehreren Faktoren zusammen. Dieses Zusammenspiel wird anhand von Abbildung 3 deutlich. 


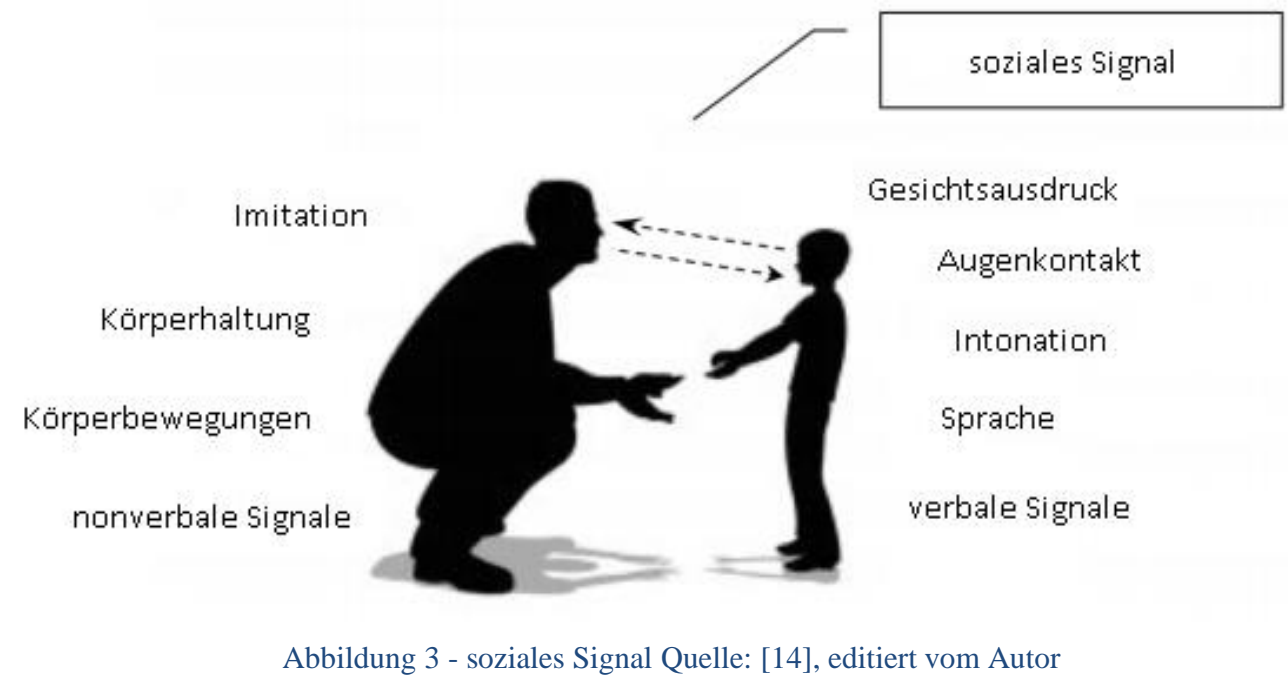

Abbildung 3 beschreibt, welche unterschiedlichen Faktoren bei einer Interaktion ausgetauscht werden. Diese multimodale* sozial-emotionale Interaktion funktioniert bei normal entwickelten Menschen korrekt. Das Wort multimodal steht hier für mehrere Modalitäten, genauer mehrere Sinneswahrnehmungen. Das heißt, ein soziales Signal setzt den Einsatz und die störungsfreie Funktionalität mehrerer Sinne voraus. Ein soziales Signal bzw. ein soziales Verhalten zu interpretieren, auszuwerten und produzieren zu können, ist Grundvoraussetzung für eine funktionierende soziale Kommunikation. Das spontane Erkennen und Auswerten einer sozialen Verhaltensweise nennt man soziale Kognition. Diese Fähigkeit ermöglicht es gesunden Menschen $\mathrm{zu}$ verstehen, dass andere Menschen Intentionen, Gedanken und Emotionen haben. Diese Fähigkeit agiert somit als Auslöser für die Empathie.

Mit sogenannten „Social Stories“ lassen sich solche Fähigkeiten trainieren. Carol Gray, welche den Begriff 1991 definierte, ist eine der bekanntesten und international anerkanntesten Autorinnen von ,Social Stories“ [15]. Diese Geschichten beschreiben Situationen, in denen das Gefühlsmotiv der Person im Mittelpunkt der jeweiligen Situation klar erkennbar und nachvollziehbar zum Ausdruck gebracht wird, sodass man sich in die Person einfühlen kann. Bei Autisten ist diese Fähigkeit beeinträchtigt. Durch ihr mangelhaftes Verständnis von Begriffen mit sozial-emotionalem Gehalt, fällt es ihnen schwer den emotionalen Kontext des Textes zu verstehen. Sie erfassen den Text somit nur wortwörtlich. Kerstin Michlo [11] spricht auch von einer mangelnden Feinabstimmung in der Interaktion.

Fehlende Empathie ist eine weitere Beeinträchtigung im autistischen Spektrum. Diese Störung wird derzeit wissenschaftlich und medizinisch mit Auffälligkeiten der Aktivitätsmuster bzw. einer Fehlfunktion der Spiegelneuronen erklärt. Was Spiegelneuronen sind und wie sie funktionieren, wird in folgendem Kapitel erörtert. 


\subsection{Spiegelneuronen}

Der folgende Abschnitt beschäftigt sich mit Spiegelneuronen und deren Auffälligkeiten bei Autisten. Die diesem Abschnitt zugrundeliegenden Informationen beruhen auf aktuellen Forschungsergebnissen und stützen sich unter anderem auf Ergebnisse der Universität von Kalifornien in San Diego [16].

\section{Was sind Spiegelneuronen?}

Spiegelneuronen sind Nervenzellen im präfrontalen Cortex ${ }^{10}$ (Stirnlappen) des Gehirns. Sie weisen bei gesunden Menschen dasselbe Aktivitätsmuster auf, wenn der Mensch einen Vorgang oder eine Handlung beobachtet, wie wenn er dieselbe Handlung selbst durchführen würde. Wenn man zum Beispiel eine Handlung beobachtet, wie das Gähnen eines anderen Menschen, werden die entsprechenden Spiegelneuronen aktiv. Sie erzeugen Signale, die dem Gehirn suggerieren, dass man die Handlung selbst durchführen

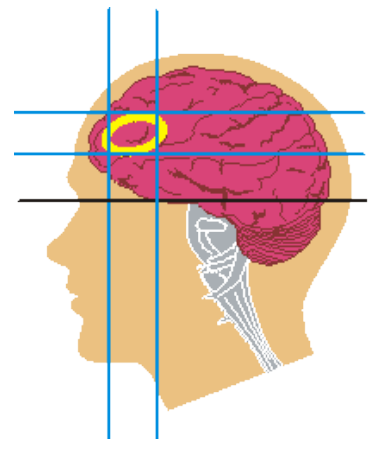

Abbildung 4 - präfrontaler Cortex Quelle: siehe Fußnote ${ }^{10}$ würde. Durch die Suggestion der Handlung entstehen im nächsten Schritt dann die Gefühle, die auch entstehen würden, wenn man die Handlung selbst durchführte. Dieser Ablauf im Gehirn ist die Grundlage für emotionale Empathie*, da automatisch nachempfunden wird, was der Gegenüber fühlt [17].

Die Spiegelneuronen von Autisten dagegen reagieren nur bei eigenen ausgeübten Handlungen, nicht aber, wenn ein anderer Mensch bei einer Handlung beobachtet wird. Das führt dazu, dass Autisten unter Umständen nicht wahrnehmen können, wenn andere Menschen sie ansprechen. Wichtiger noch ist die damit einhergehende Unfähigkeit, Handlungen und Bewegungen eines anderen Menschen zu imitieren und nachzuahmen [18]. Intakte Spiegelneuronen sind somit eine wichtige Grundlage für das Empathie-Empfinden des Menschen. Gestützt auf die Ergebnisse der Universität in San Diago fehlt Autisten demnach das nötige Einfühlungsvermögen, wenn sie andere Menschen beobachten.

Bei der Empathie muss hierbei zwischen der kognitiven und emotionalen Empathie unterschieden werden. Die kognitive Empathie befasst sich mit dem Verständnis davon, welche Handlung ein Gegenüber ausübt. Die emotionale Empathie ermöglicht das Nachempfinden der Gefühle des anderen bei dieser Handlung. Beide Funktionen weisen bei Autisten Defizite auf, die es durch ein intensives Training auszugleichen gilt. Im Kapitel der

\footnotetext{
${ }^{10}$ Quelle: http://www.ipn.at/ipn.asp?BRQ - Zugriff: 31.017.2014
} 
Konzeptentwicklung wird eingehend auf den therapeutischen Ansatz eingegangen, Empathie und das soziale Verständnis durch Imitation zu trainieren.

\section{Human-Robot-Interaction (HRI)}

Das folgende Kapitel greift, mit Fokus auf die aktuelle Entwicklung der Human-RobotInteraction, das Themengebiet der Socially Assistive Robotics (SAR) auf und legt die darin einzuordnende Rolle humanoider Roboter, als therapeutisches Mittel im autistischen Spektrum, dar. In Unterkapitel 3.4 wird der Einsatz des humanoiden NAO-Roboters zur Förderung autistischer Kinder diskutiert. Eine Auswertung der Betrachtung wird im Fazit festgehalten.

\subsection{Definition der HRI}

Eine treffende Definition des Inhalts der Human-Robot-Interaction geht aus einer Fallstudie des International Symposium on Robotics and Intelligent Sensors 2012. (IRIS 2012) von Syamimi Shamsuddin von der Technischen Universität Malaysia hervor. Sie lautet:

\section{„HRI is simply defined as the dynamically changing relationship between humans and intelligent robots. “[4]}

Diese Thematik ist ein weltweites hochaktuelles und multidisziplinäres Forschungsfeld. Es werden jährlich mehrere hochrangige Konferenzen und Symposien zu diesem Thema abgehalten, die dem internationalen Austausch und der Veröffentlichung aktueller Fallstudien und Forschungsergebnisse dienen. Hierzu zählen unter anderem die Konferenzen ICSR ${ }^{11}$, $\mathrm{HRPR}^{12}$ und IEEE (RO-MAN) ${ }^{13}$. In Zusammenarbeit mit dem $\mathrm{ACM}^{*}$ und dem IEEE* wurde 2006 die derzeit größte Konferenz, die ACM/IEEE HRI ${ }^{14}$, ins Leben gerufen.

Ein übergeordnetes Ziel der HRI ist es, die Interaktion und Kommunikation zwischen Mensch und Roboter so intuitiv wie möglich zu gestalten. Ein besonderer Schwerpunkt ist die Entwicklung von Assistenz-Robotern im sozialen Bereich.

\subsection{Socially Assistive Robotics (SAR)}

Das Themengebiet Socially Assistive Robotics (SAR), ist ein zunehmend wachsender Bereich innerhalb der HRI. Der englischsprachige Begriff übersetzt so viel wie

\footnotetext{
${ }^{11}$ ICSR - International Conference on Social Robotics, seit 2009

12 HRPR - International Conference on Human-Robot Personal Relationships, seit 2008

${ }^{13}$ IEEE RO-MAN - IEEE International Symposium in Robot and Human Interactive Communication, seit 1992

${ }^{14}$ ACM/IEEE HRI - International Conference on Human-Robot Interaction, seit 2006
} 
„soziale/gesellschaftliche Assistenz-Robotik“. Im weiteren Verlauf der Arbeit wird die Abkürzung SAR benutzt. Der Schwerpunkt solcher Assistenz-Roboter liegt in der Unterstützung und Interaktion mit kranken, hilfsbedürftigen und älteren Menschen [19, 20]. Sie werden im Bildungswesen eingesetzt und helfen Menschen bei Rehabilitationsmaßnahmen [21]. Der in dieser Arbeit zu beleuchtende Bereich der SARSysteme beschäftigt sich speziell mit der sozialen Interaktion humanoider Roboter und deren therapeutischem Einsatz im autistischen Spektrum.

\subsection{Assistenz-Roboter im autistischen Spektrum}

Wie schon im Ansatz in der Einleitung erwähnt, belegen zahlreiche Studien, dass autistische Kinder eine Affinität gegenüber mechanischen und technischen Objekten aufweisen [22-25]. Sie zeigen größeres Interesse an solchen Objekten als an anderen Menschen. Das liegt daran, dass in diesem Fall Roboter nicht so kompliziert sind wie Menschen [10]. Damit ist unter anderem das Fehlen der komplexen Mimik und Gestik jener Roboter gemeint. Ein Grundgedanke von SAR-Systemen im autistischen Spektrum ist die Förderung der sozialen Interaktion in Begleitung von Eltern, Geschwistern oder Therapeuten [26]. Hierbei macht man sich das höhere Konzentrationslevel der Kinder, wenn sie mit Robotern interagieren, zunutze [9]. Ein weiteres Ziel ist es, eine Beziehung zwischen dem Kind und dem Roboter herzustellen. Diese Beziehung ist notwendig, um angestrebte therapeutische Ziele zu erreichen [26]. Vielversprechend sind Studien, in denen die Herstellung des Augenkontaktes zwischen Kind und Roboter im Mittelpunkt steht [27]. Das Herstellen des Augenkontaktes ist eine erste Initiierung einer sozialen Interaktion und somit eine Grundvorrausetzung für eine funktionierende Kommunikation [28, 27]. 
Es gibt weltweit verschiedene Arten von Robotern, die in Therapien mit autistischen Kindern eingesetzt werden. Das europäische AuRoRA Projekt [29] benutzt seit 1999 z.B. einfache mobile Roboter, welche Schlüsselaspekte der menschlichen Kommunikation

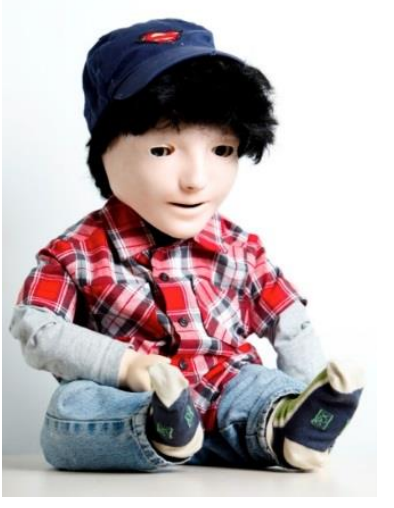

Abbildung 6 - KASPAR Quelle: siehe Fußnote ${ }^{17}$ und Interaktion, wie Augenkontakt und Sprecherwechsel ${ }^{15}$, unterstützen und fördern. Mit diesen mobilen Robotern werden autistische Kinder durch komplexe soziale Interaktionen

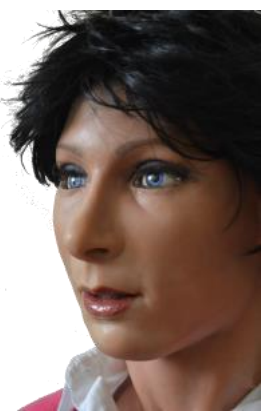

Abbildung 5 - The FACE Android Quelle: siehe Fußnote ${ }^{16}$ geführt und angeleitet. Der Roboter FACE (Abbildung 5) der Universität von Pisa ist entwickelt worden, um das menschliche Gesicht so getreu wie möglich nachzuempfinden. In Studien wurden die Kinder angehalten, auf Fotos von menschlichen Gesichtern zu zeigen, die denselben Gesichtsausdruck haben wie der Roboter FACE. Bei jeder neuen Mimik verbalisierte der Roboter diese zusätzlich. Ein anderes Szenario sieht vor, dass die Kinder FACE ein Foto zeigen und der Roboter den gezeigten Gesichtsausdruck annimmt [30]. Das Ziel dieser Therapie ist es, dass die Kinder die Generalisierung der Informationen, die sie in der Therapie lernen, auf andere Situationen übertragen können. Mit Generalisieren ist hier gemeint, aus einer komplexen Gesichtsmimik mit den dazugehörigen verbalen Äußerungen, wie z.B. das Lachen eines Menschen, die Emotion „Freude“ zu extrahieren und zu verstehen. Auch der von der britischen Universität in Hertfordshire entwickelte Roboter KASPAR (Abbildung 6) kann einfachste Bewegungen mit den Armen und Gesichtsausdrücke, ohne die Komplexität realer menschlicher Gesichtszüge, repräsentieren [29].

Im Laufe der Zeit haben sich drei sinnvolle Einsatzformen entwickelt, die ein Roboter bei einer Therapie mit autistischen Kindern einnehmen kann und die aktuell praktiziert werden. Der Roboter kann erstens die Rolle eines Lehrers mit Autorität übernehmen. Mit dieser Rolle kann soziales Verhalten demonstriert und eine Interaktion durchgeführt werden. Er kann in zweiter Instanz als Mediator zwischen Therapeut und Kind dienen, um Verhaltensweisen und Aufforderungen an das Kind zu vermitteln. Und zum Dritten kann er als Medium dienen, um Emotionen oder Ziele des Kindes mit Hilfe der ,gestützten Kommunikation“** zu vermitteln, wobei letzteres nicht sehr häufig praktiziert wird [31].

\footnotetext{
15 gemeint ist die intuitive Verteilung der Gesprächsbeiträge auf die Gesprächsteilnehmer

${ }^{16}$ Bildquelle: http://www.faceteam.it/wp-content/uploads/2012/07/FACE-Profile.png, Zugriff am 23.07.14

${ }^{17}$ Bildquelle: http://blogs.herts.ac.uk/research/files/2011/06/Kaspar-0021.jpg, Zugriff am 23.07.14
} 
Viele Roboter werden gezielt für spezielle Therapien entwickelt und als Prototyp für diese Therapien konstruiert. Die physische Erscheinung der Roboter ist dabei oft auf den Therapieschwerpunkt angepasst. Nicht-humanoide Roboter, wie oben beschrieben, sind somit nur für bestimmte Interaktionstypen vorgesehen. Obwohl einige generelle Designeigenschaften für Therapieroboter vorteilhaft sind, gibt es dennoch keine festgelegte Richtlinie, wie Roboter auszusehen haben, die autistischen Kindern helfen sollen. Robin et al. haben in ihrer Studie den Einfluss und die Wichtigkeit des Aussehens eines Roboters in der Autismus-Therapie untersucht. Sie kamen zu dem Ergebnis, dass der Roboter humanoide Formen haben sollte, aber der Komplexität und dem Detailreichtum eines echten Menschen zu nicht nahe kommen darf [32]. Bevor nun die Beschreibung des NAO-Roboters erfolgt, ist es sinnvoll darzustellen, was einen humanoiden Roboter ausmacht.

\section{Was sind humanoide Roboter?}

Mit dem Begriff eines humanoiden Roboters ist eine Konstruktion gemeint, die dem menschlichen Körper nachempfunden ist. So besteht ein humanoider Roboter meist aus einem Kopf, einem Torso, zwei Armen und zwei Beinen. Abgewandelte Formen bestehen beispielsweise nur aus dem Oberkörper. Humanoide Roboter werden entwickelt, um dem Menschen ein helfender Begleiter $\mathrm{zu}$ sein und ihm unterstützend zur Seite zu stehen. Humanoide Roboter ermöglichen eine Generalisierung einzelner Verhaltensweisen [33], so dass sie den autistischen Kindern separat beigebracht werden können. Das heißt, es lassen sich komplexe Bewegungen in einzelne Schritte aufteilen. Mit einem humanoiden Roboter können am besten Emotionen oder soziale Verhaltensweisen imitieren und generalisiert werden. Das Thema „Imitation“, welches therapeutisch eine wichtige Rolle spielt, wird weiter unten noch einmal aufgegriffen und erläutert.

Im nun folgenden und letzten Abschnitt des Kapitels der Human-Robot-Interaction werden Ergebnisse aus vier ausgewählten Fallstudien aus Malaysia, Frankreich, China und den USA bezüglich der HRI mit dem NAO-Roboter in der Autismus-Therapie dargelegt. Dazu ist es notwendig, den in dieser Arbeit verwendeten humanoiden NAO-Roboter zuerst so hinreichend zu beschreiben, dass der Mehrwert seines derzeitigen Einsatzes in der AutismusTherapie deutlich wird. Eine detaillierte ingenieurstechnische Betrachtung der physikalischen und technischen Grundlagen des NAO-Roboters erfolgt im Kapitel 4ff. 


\subsection{HRI mit dem NAO-Roboter}

Mit seinen $57,2 \mathrm{~cm}$ ist der humanoide NAO-Roboter der französischen Firma Aldebaran Robotics ein verhältnismäßig kleiner Roboter. Er wiegt 5,2 kg und hat die Gestalt eines Kleinkindes. Mit seinen 25 Freiheitsgraden (siehe Kapitel 4.1.5) ist er in der Lage, eine große Anzahl von menschlichen Gesten abzudecken.
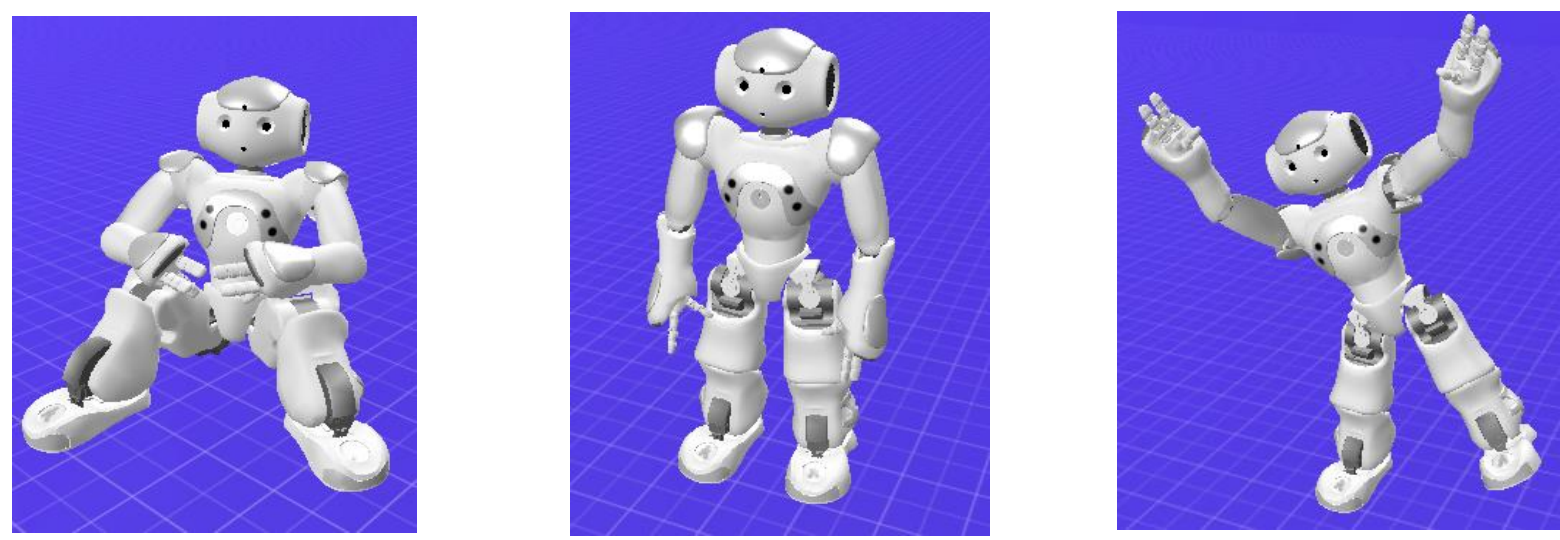

Abbildung 7 - Bewegungsspielraum des NAO-Roboters

Der NAO-Roboter entspricht der Anforderung menschlich auszusehen, aber nicht zu detailreich und komplex zu sein, wie sie Robin et al. in ihrer Studie beschrieben haben (Abschnitt 3.3). Weiterhin hat der NAO-Roboter die Fähigkeit, zu sehen und zu erkennen, zu hören und $\mathrm{zu}$ verstehen sowie $\mathrm{zu}$ sprechen und $\mathrm{zu}$ betonen. Mit diesen Fähigkeiten und Spezifikationen sind ihm die besten Voraussetzungen zur Kommunikation und Interaktion gegeben. Gekoppelt mit einfachen Bewegungen kann er z.B. Verhaltensweisen imitieren oder dazu auffordern. Durch seine serienmäßige, verhältnismäßig kostengünstige Vermarktung durch Aldebaran Robotics ist er nicht mehr aufwendig als Prototyp herzustellen, sondern kann durchaus auch von kleineren Therapiezentren erworben und eingesetzt werden. Nachfolgend werden vier Studien beschrieben, die später als Grundlage für die Konzeptentwicklung herangezogen werden. 


\section{Studie 1:}

„Eye contact is also potentially an ideal model system for studying the neural, cognitive and developmental basis of a typical social interaction and communication in Autism Spectrum Disorders ,, [27]

An der Technischen Universität von Malaysia (UiTM) sind in Kooperation mit dem IEEE im Jahr 2012 einige wissenschaftliche und therapeutische Anwendungsszenarien mit dem NAO evaluiert worden. Die Evaluationen werden innerhalb des eigens von der UiTM gegründeten Robot-based Intervention Program (RBIP*) abgehalten. In der hier beschriebenen Studie wurde das Konzentrationslevel anhand des Aufrechterhaltens des Blickkontaktes zum Roboter im Unterschied zur normalen Aufmerksamkeit im Klassenraum gemessen. Getestet wurden zwölf Kinder mit Autismus. Die Studie zeigt, dass die durchschnittliche Dauer des Blickkontaktes bei Interaktion mit dem NAO-Roboter höher war als im Klassenraum mit anderen Kindern [27], siehe Abbildung 8.

Vergleicht man in Abbildung 8 die mittlere Spalte mit der rechten, ist die Zeitdifferenz gut zu erkennen.

\begin{tabular}{|ccc|}
\hline Child ID & Total Average Eye Contact Time (seconds) In RBIP & $\begin{array}{c}\text { Total Average Eye Contact Time } \\
\text { In Classroom (seconds) }\end{array}$ \\
\hline 1 & 311.29 & 208.83 \\
2 & 381.17 & 227.57 \\
3 & 430.92 & 400.32 \\
4 & 374.15 & 311.99 \\
5 & 454.16 & 410.35 \\
6 & 366.06 & 336.56 \\
7 & 362.89 & 274.66 \\
8 & 500.85 & 334.87 \\
9 & 336.98 & 232.17 \\
10 & 168.31 & 111.22 \\
11 & 360.05 & 260.1 \\
12 & 415.03 & 356.59 \\
\hline
\end{tabular}

Abbildung 8 - Zeit des Blickkontakts im Vergleich RBIP und Klassenraum Quelle: [17]

\section{Studie 2:}

„[...] children with ASD present more stereotype behavior in the classroom interaction and showed less stereotype behavior during the interaction in RBIP. Stereotyped behavior can be reduced [...] “ [34] 
Eine weitere Studie der UiTM untersuchte, ob eine Reduzierung des stereotyp repetitiven Verhaltens autistischer Kinder bei Interaktion mit dem NAO erzielt werden kann. Getestet wurden sechs Kinder, die an Autismus leiden.

Unter stereotyp repetitivem Verhalten versteht man unteranderem folgende Verhaltensweisen:

- vermeidet konsequent Blickkontakt, wenn dieser hergestellt wird

- starrt auf Hände, Objekte oder Gegenstände in der Umgebung für mindestens fünf Sekunden

- schnipst mit den Fingern vor den Augen für mindestens fünf Sekunden

- dreht sich in Kreisen

- dreht Objekte, die nicht fürs Drehen gedacht sind

- macht hochtönige Geräusche

- läuft auf Zehenspitzen

Als Quelle für die oben aufgezählten Eigenschaften ist die Gilliam Autism Rating Scale (GARS-2) zu nennen. Diese Wertungsskala bietet 42 Punkte zur Identifizierung von Auffälligkeiten im autistischen Spektrum [35].

Der Abstand des blauen Graphen zur gestrichelten grünen Linie in Abbildung 9 zeigt, dass es möglich ist, das stereotype Verhalten zu verringern [34].

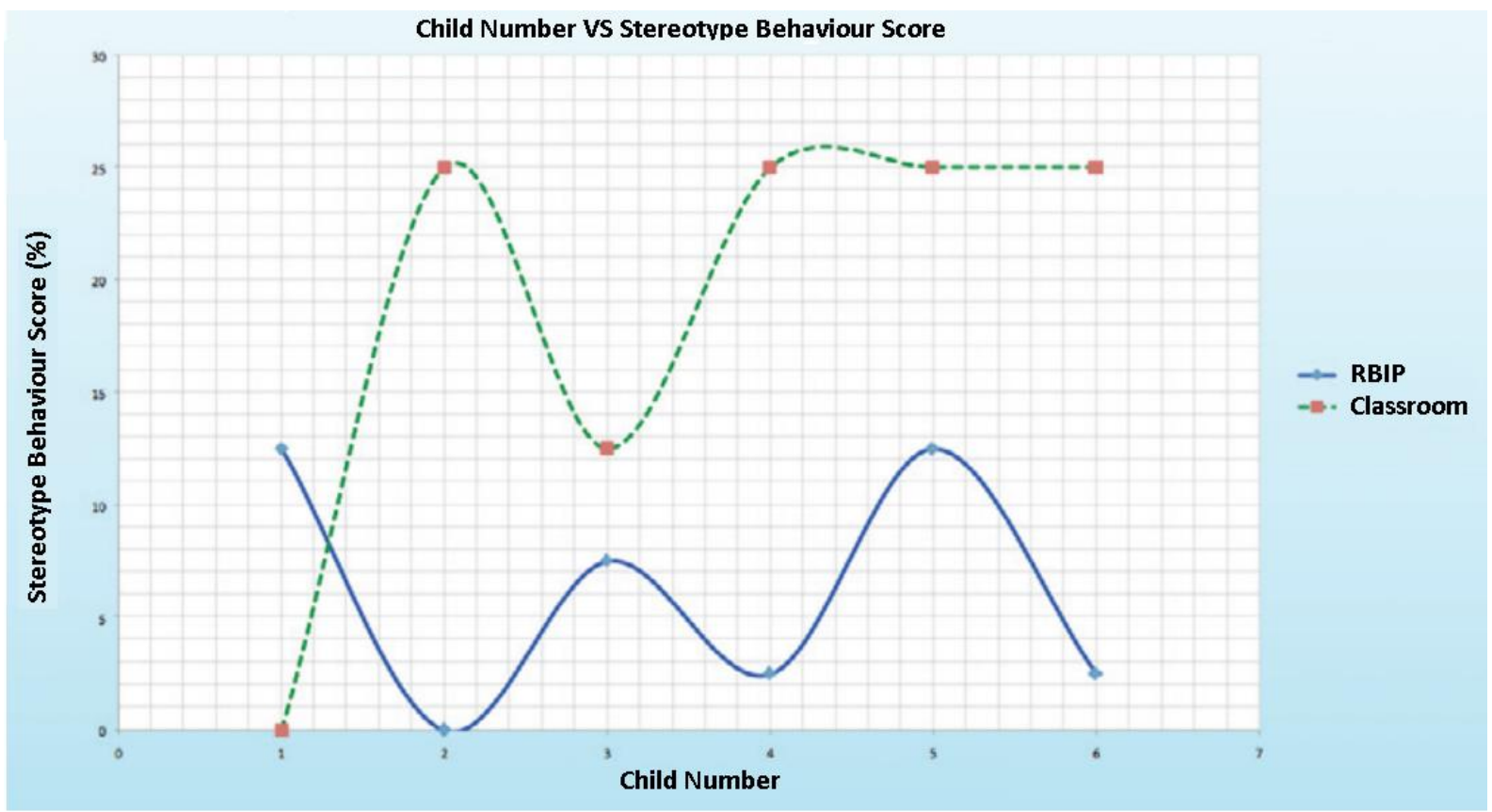

Abbildung 9 - Stereotyp Verhalten RBIP gegenüber Klassenraum Quelle: [18], editiert vom Autor 


\section{Studie 3:}

„The game motivates the children to pay attention to the robot. They are explicitly and/or implicitly asked for the emotions conveyed by the robot" [36]

Mustafa S. Erden von der École Nationale Supérieure de Techniques Avancées (ENSTA) in Paris erarbeitete 2013 Körperhaltungen mit dem NAO-Roboter, die eindeutig einer Emotion zuzuordnen sind. Er kommt zu dem Schluss, dass sich von den sechs Basisemotionen (glücklich, überrascht, wütend, ängstlich, traurig und angeekelt) drei am anschaulichsten mit dem NAO manifestieren lassen. Diese drei sind wütend, traurig und glücklich [37]. Erden bediente sich der Ausarbeitungen von Culson [38]. Culson hat die von Ekman und Friesen [39] qualitativ beschriebenen sechs Basisemotionen quantitativ so aufbereitet, dass die Körperhaltungen nun in Gelenkinformationen und Winkelpositionen vorlagen. Diese hat Erden für seine Umsetzung mit dem humanoiden NAO-Roboter genutzt. Abbildung 10 zeigt das von Culson entwickelte Modell für die drei Emotionen Glück, Traurigkeit und Wut. Dabei sind die Haltungen jeweils von der Seite, von hinten und von vorne zu sehen. Abbildung 11, 12 und 13 auf der nächsten Seite zeigen, wie Erden die Emotionen mit dem NAO realisiert hat.

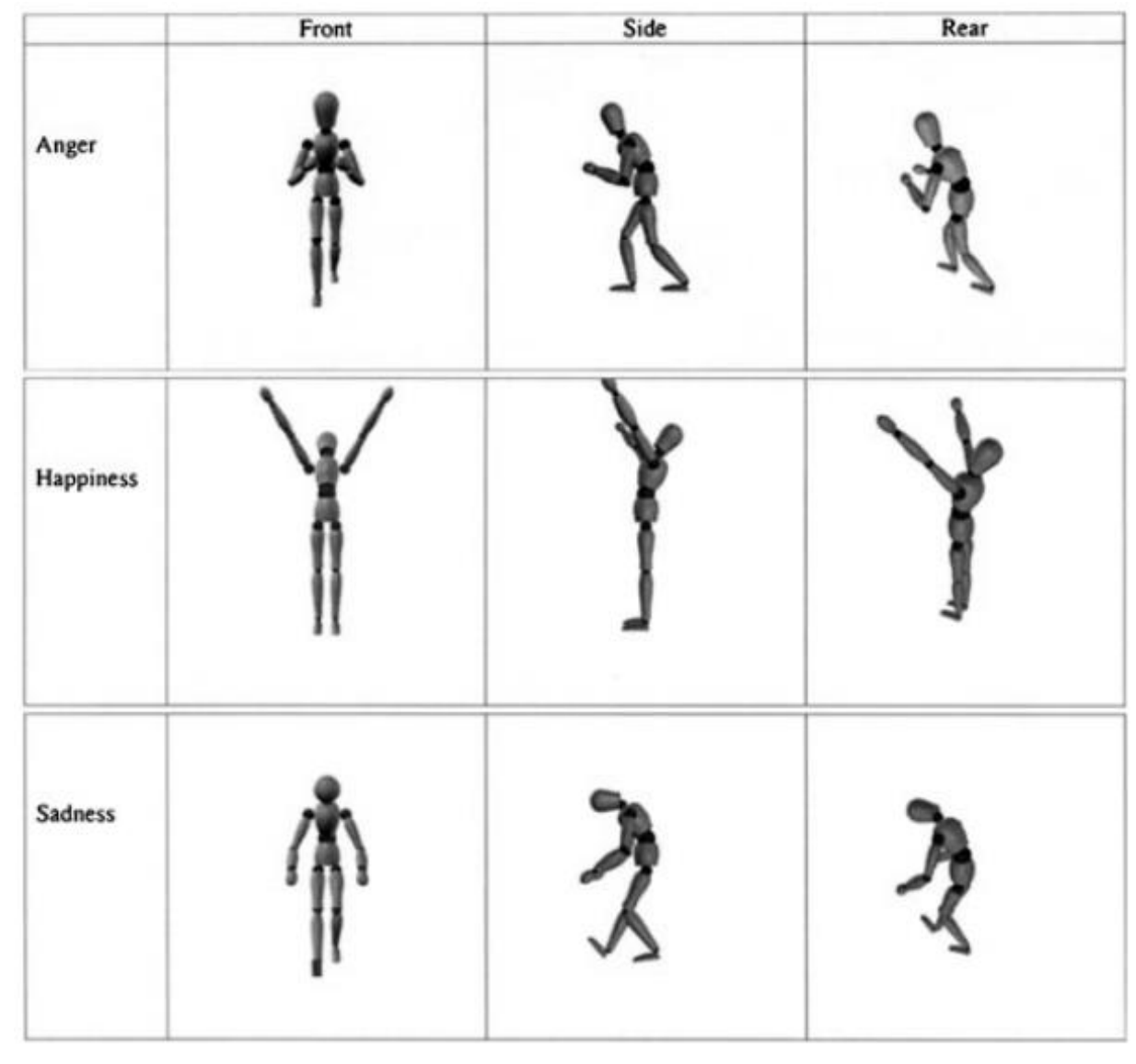

Abbildung 10 - drei Basisemotionen von Culson [14] 


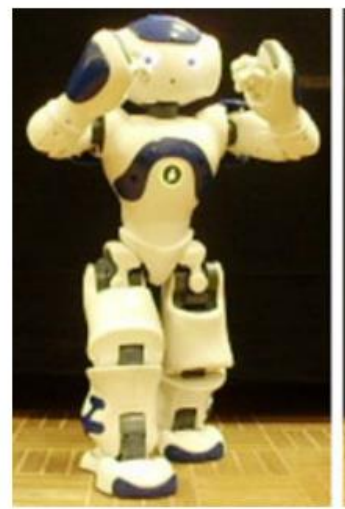

anger_04

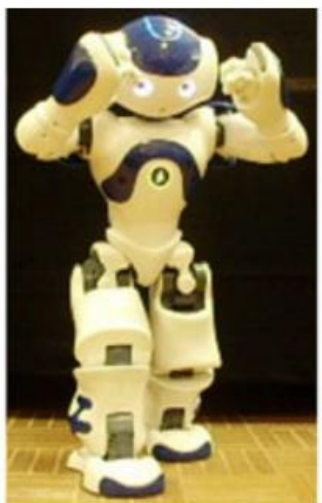

anger_12

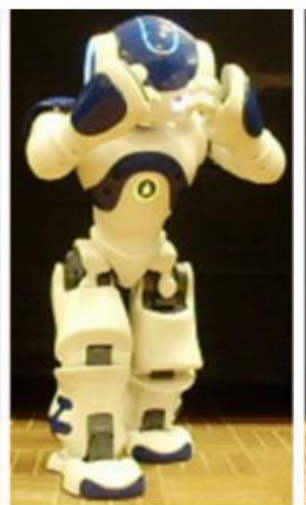

anger_32

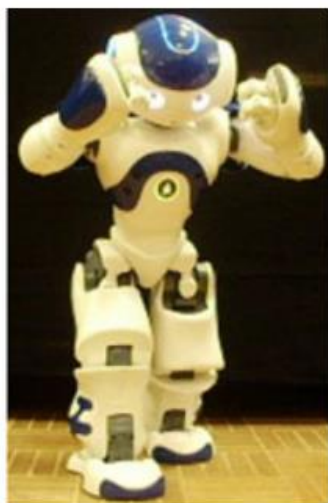

anger_28

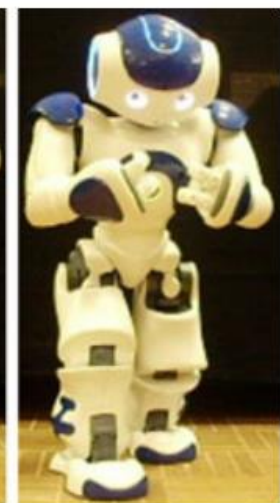

anger_30

Abbildung 11 - fünf ausgewählte Körperhaltungen für „wütend“ Quelle: [14]

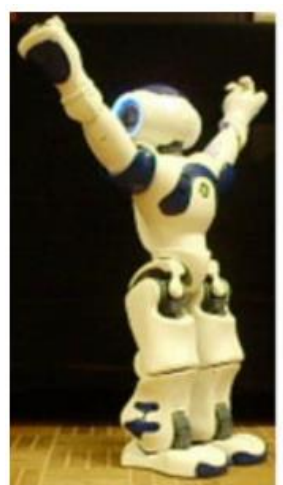

happiness_13

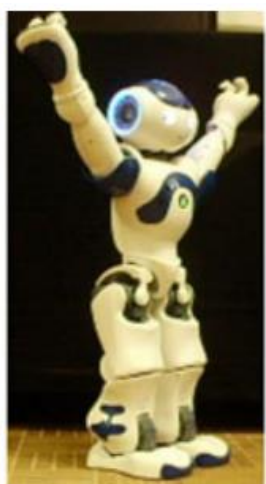

happiness_01
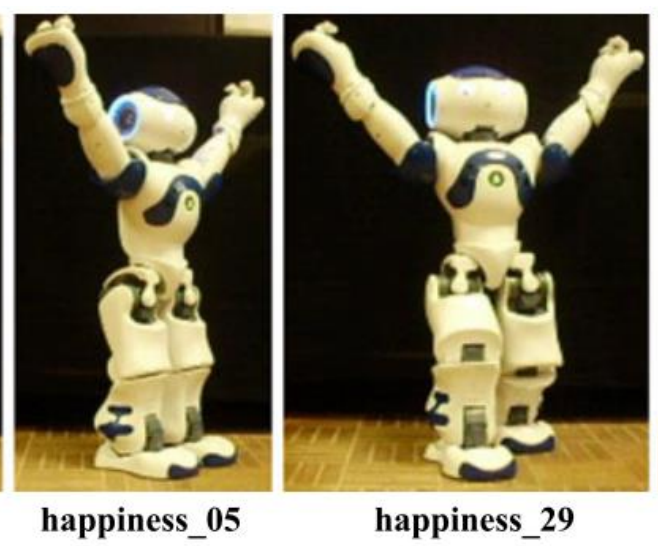

happiness_29

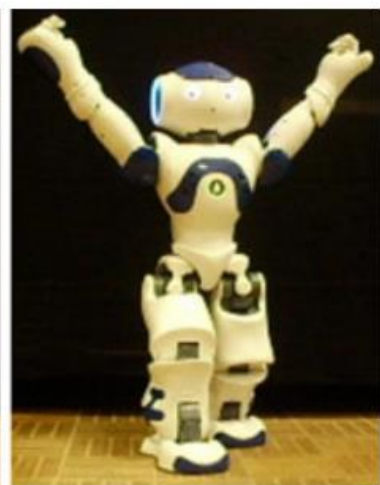

happiness_21

Abbildung 12 - fünf ausgewählte Körperhaltungen für ,glücklich“ Quelle: [14]

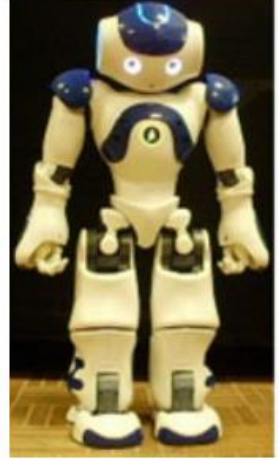

sadness_04

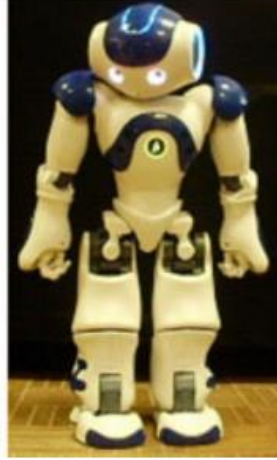

sadness_16

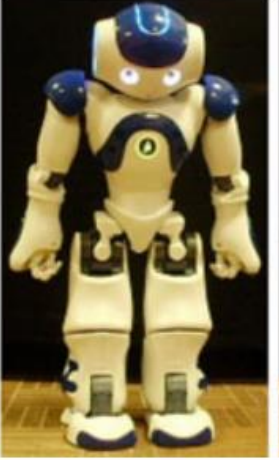

sadness_08

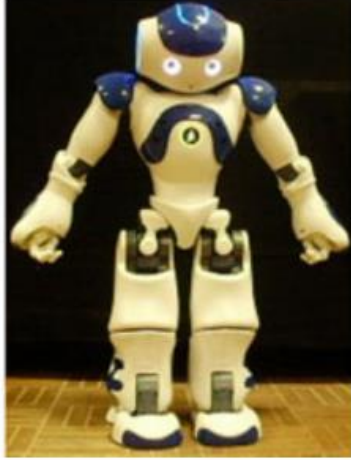

sadness_03

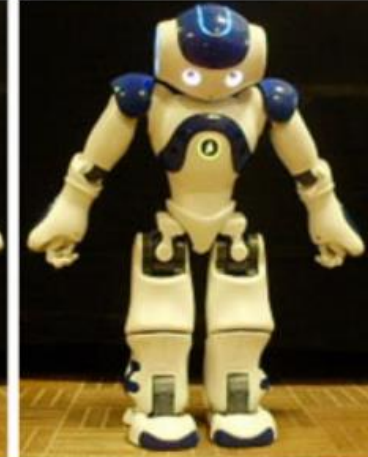

sadness_07

Abbildung 13 - fünf ausgewählte Körperhaltungen für "traurig" Quelle: [14]

Das Zitat zu Beginn dieses Abschnittes zeigt einen Ausblick, den Erden im Fazit seiner

Evaluation gegeben hat. Es deutet darauf hin, dass es denkbar wäre, diese Körperhaltungen in ein Spiel mit dem NAO zu implementieren, um damit autistischen Kindern die grundlegenden Emotionen nahezubringen. 


\section{Studie 4:}

„Imitation training has been known to be one of the important and effective therapeutic interventions for improving social skills of autistic children. " [40]

Obiges Zitat stammt aus einer Studie, die im Rahmen des im August 2013 abgehaltenen IEEE RO-MAN Symposiums veröffentlicht wurde. Es besagt, dass das Imitieren eine der wichtigsten therapeutischen Behandlungen für die Verbesserung der sozialen Fähigkeiten autistischer Kinder darstellt. Jungsik Hwang entwickelt in dieser Studie einen Ansatz für Interaktionsszenarien in der Autismus-Therapie weiter. Diese Szenarien beziehen sich auf einzelnen therapeutischen Sitzungen, in denen nicht nur der Kontakt zwischen Kind und Roboter, sondern die Interaktion zwischen dem NAO, dem autistischen Kind und dem Therapeuten aufgegriffen werden. Adaptiert wurden nur Therapiekonzepte, in denen mit dem Kind Imitationen geübt wurden. Der Schwerpunkt in Hwangs Studie liegt nicht in der Förderung der Beziehung zwischen Kind und Roboter. Er versucht vielmehr die Beziehung zwischen einem Menschen (Therapeut) und dem Kind zu fördern. Dazu hat er ein in mehreren vorherigen Studien benutztes zweiteiliges Interaktionsmodell $\mathrm{zu}$ einem dreiteiligen Interaktionsmodell weiterentwickelt [37]. Siehe dazu Abbildung 14 und 15.

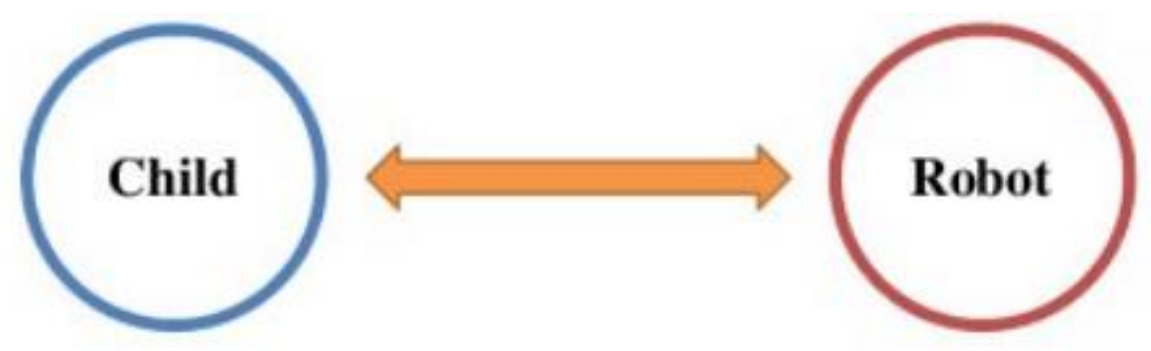

Abbildung 14 - zweiteiliges Interaktionsmodell Quelle: [22]

In Abbildung 14 agiert der Roboter autonom mit dem Kind. Der Therapeut nimmt in diesem Szenario keinen Einfluss auf das Imitationsverhalten des Kindes. 


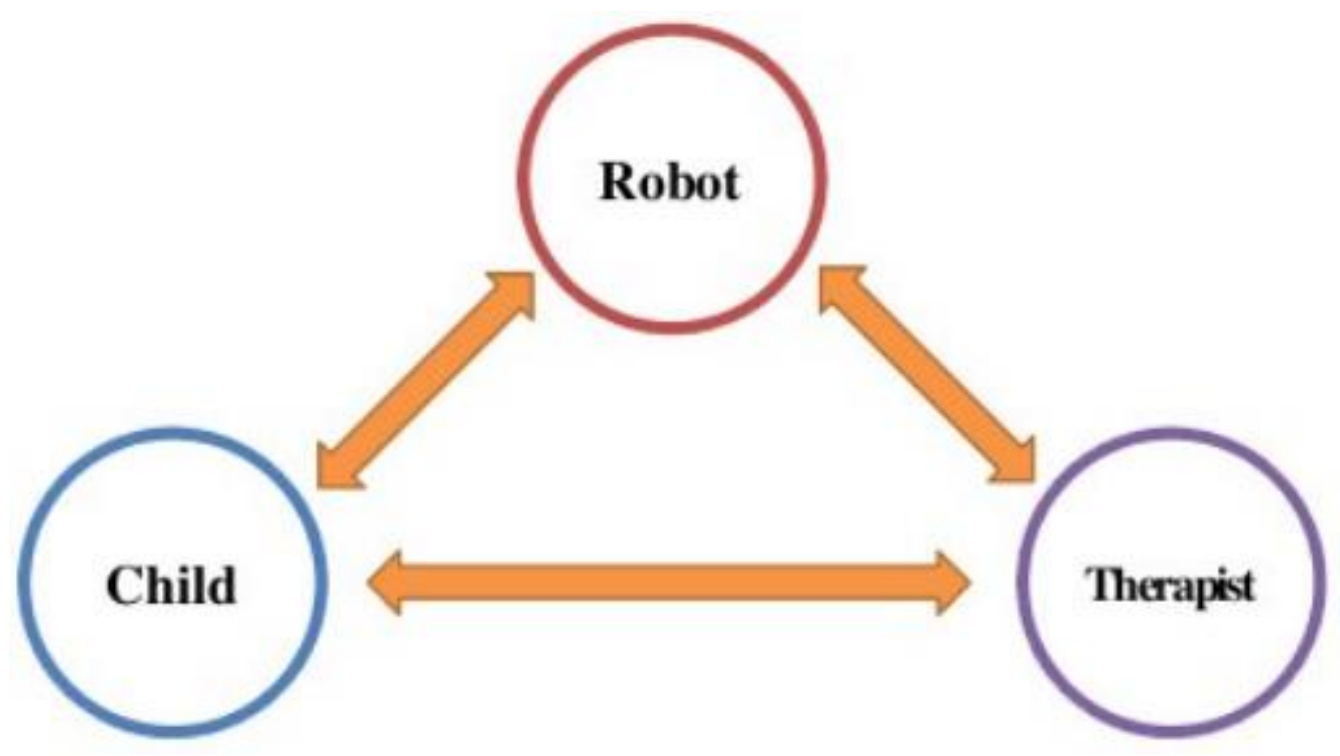

Abbildung 15 - dreiteiliges Interaktionsmodell Quelle: [22]

Abbildung 15 zeigt den Roboter als Mediator zwischen Kind und Therapeut. Dieses Interaktionsmodell unterstützt die Förderung der sozialen Kommunikation durch ein Erreichen der geteilten Aufmerksamkeit.

Geteilte Aufmerksamkeit und Imitation, eingebettet in spielerische Anwendungen, versprechen die besten therapeutischen Ergebnisse [41]. Daraus lässt sich schließen, dass die geteilte Aufmerksamkeit ein weiterer Schlüsselaspekt für die soziale Kommunikation ist. Dieses Interaktionsmodell wird unter anderem der Konzeption der Anwendung in dieser Arbeit als Basis dienen.

\subsection{Zwischenfazit}

Zusammenfassend ist festzuhalten, dass in den letzten Jahren in der Therapie mit nichthumanoiden oder halb-humanoiden Robotern nennenswerte Fortschritte erzielt wurden. Der Einsatz humanoider Roboter in der Autismus-Therapie steht erst am Anfang der Entwicklung. Der NAO-Roboter zeigt sich mit seiner Bauweise, wie durch diverse Studien belegt ist [42, 24, 43, 44, 37], als ideales Medium, um in solchen Therapien unterstützend eingesetzt werden zu können.

Abgeleitet aus dem obigen Kapitel kann ein Einsatz des NAO-Roboters im therapeutischen Bereich in drei Szenarien unterteilt werden. Zum einen ist es möglich, den NAO als Medium, ferngesteuert durch den Therapeuten, mit dem Kind interagieren zu lassen. Die zweite Form sieht den Roboter in der Rolle als sozialen Freund, der autonom mit dem Kind auf sehr einfache Weise interagiert. Die dritte und letzte Möglichkeit sieht den Roboter als 
eigenständigen Mediator zwischen dem Therapeuten und dem Kind [45]. Diesen Ansatz wird der Autor verfolgen.

Ein weiterer Vorteil ist, dass der NAO für jedes Kind speziell programmiert werden kann. Welche Möglichkeiten der Programmierung es gibt und welche technischen Details der NAO noch zu bieten hat, wird im folgenden Kapitel ausführlich beschrieben.

\section{Technische Grundlagen}

Da der humanoide Roboter NAO (Version 4.0 H25) für Lehr- und Forschungszwecke entwickelt wurde, bietet er eine große Zahl an Hardware- und Softwarekomponenten. Nachfolgend werden diese beschrieben. Dabei sind Komponenten, die hauptsächlich der Human-Robot-Interaction dienlich sind und eine wichtige Rolle in der Konzeptumsetzung spielen, mit einer separaten Überschrift versehen. Im Softwareteil dieses Kapitels werden drei Entwicklungsumgebungen vorgestellt, mit denen der NAO programmiert werden kann. Der Autor wird diese drei bewerten sowie die Vor- und Nachteile aufzeigen.

\subsection{Hardware}

Das Herzstück des Roboters ist ein 1,6 GHz Intel Atom Z530 Prozessor*, der im Kopf platziert ist. Unterstützt wird der Prozessor durch ein Gigabyte Arbeitsspeicher und zwei Gigabyte internen Flashspeicher*. Ein microSDHC*-Erweiterungssteckplatz für bis zu acht Gigabyte externen Flashspeicher ist vorhanden. Der eingebaute Ionen-Akkumulator* versorgt den Roboter bei intensiver Nutzung für ca. 60 Minuten mit Strom.

Die Kommunikation mit dem NAO erfolgt wahlweise über einen 1GBit Ethernet Port* am Hinterkopf oder über das interne WLAN-Modul mit der Spezifikation $802.11 \mathrm{~b} / \mathrm{g} / \mathrm{n}$. Der Roboter ist in der Lage sich mit einem WLAN-Hotspot* zu verbinden oder selbst einen Hotspot zu erzeugen. Das sogenannte Tethering* ist von Vorteil, wenn sich kein Hotspot in der Nähe befindet und trotzdem eine kabellose Verbindung zum Roboter nötig ist. Als weitere Kommunikationsmöglichkeit besitzt der NAO in den Augenpartien Infrarotschnittstellen zum Senden und Empfangen von Infrarotsignalen*. Zusätzlich kann die Umgebung mit zwei an der Vorderseite des Torsos verbauten Ultraschallsensoren untersucht und analysiert werden. Zwei intern verbaute Gyro- und ein Beschleunigungssensor ermöglichen es, Rotationen (Drehung um die X-, Y- und Z-Achse) und Translationen (Bewegung auf der X-, Y- und Z-Achse) zu messen. 


\subsubsection{Taktile und mechanische Sensoren}

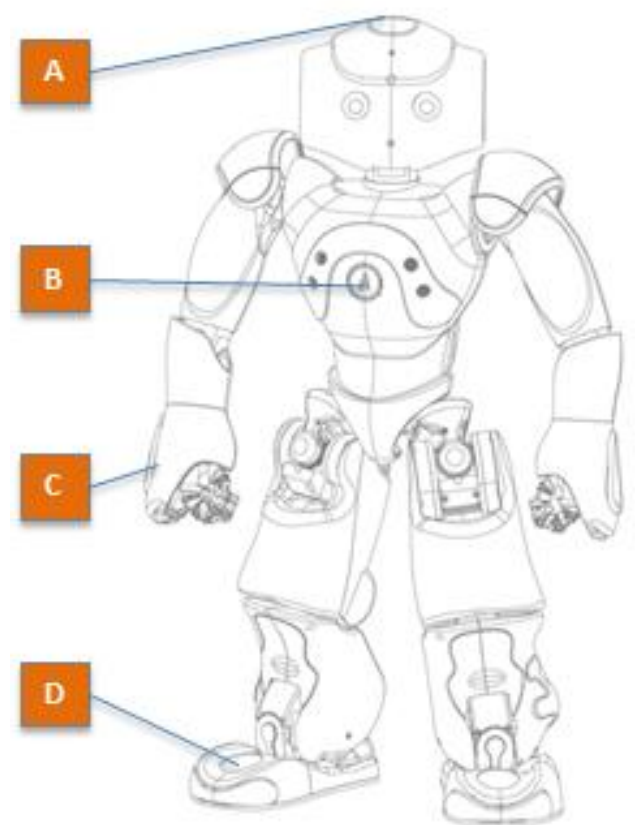

Abbildung 16 - taktile und mechanische Sensoren Quelle: Aldebaran Robotics

Der NAO-Roboter hat insgesamt elf Sensoren, neun taktile (kapazitiv) und drei mechanische. Diese werden in die vier Bereiche Kopf, Brust, Hände und Füße eingeteilt. Zu den drei mechanischen Sensoren gehören der CHEST-Button $\quad$ в und die beiden Fuß-Bumper D . Diese Drucksensoren funktionieren wie einfache ON/OFF Schalter. Die Fuß-Bumper befinden sich an der Spitze des Fußes. (Abbildung 18)

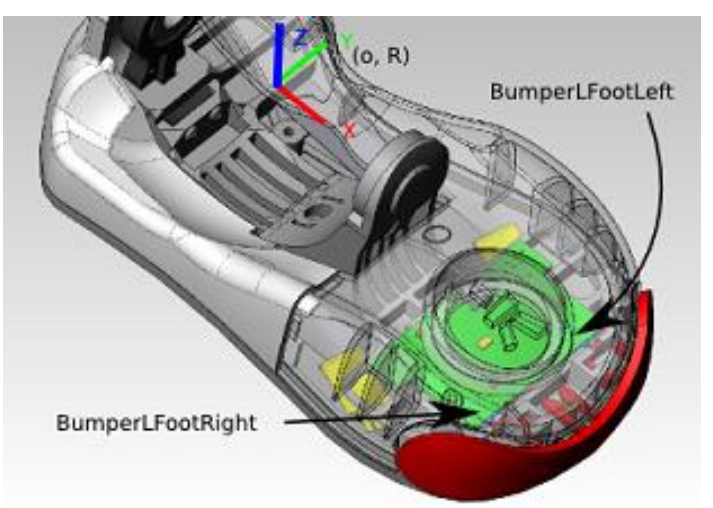

Abbildung 18 - mechanischer Schalter am Fuß Quelle: Aldebaran Robotics

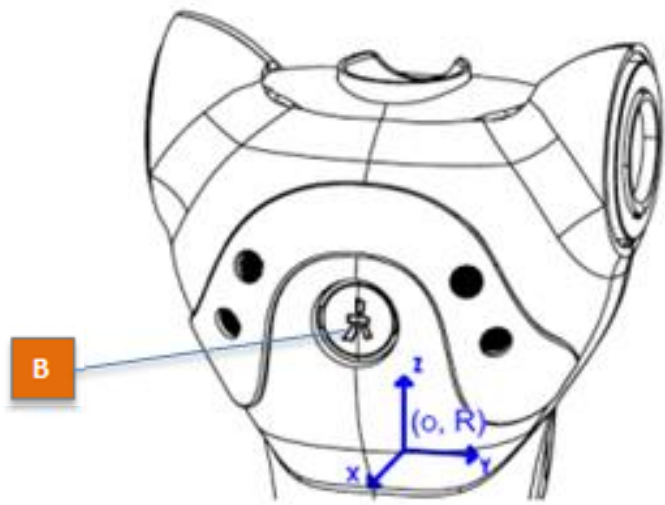

Abbildung 17 - CHEST-Button - Knopf auf der Brust Quelle: Aldebaran Robotics 
Des Weiteren gibt es am Kopf drei taktile Sensoren. Die drei Kopfsensoren A sind in Abbildung 19 mit rear, middle und front gekennzeichnet. Jeder dieser Sensoren kann einzeln abgefragt und ausgewertet werden. Es reicht eine kurze Berührung mit dem Finger, um den Sensor auszulösen.

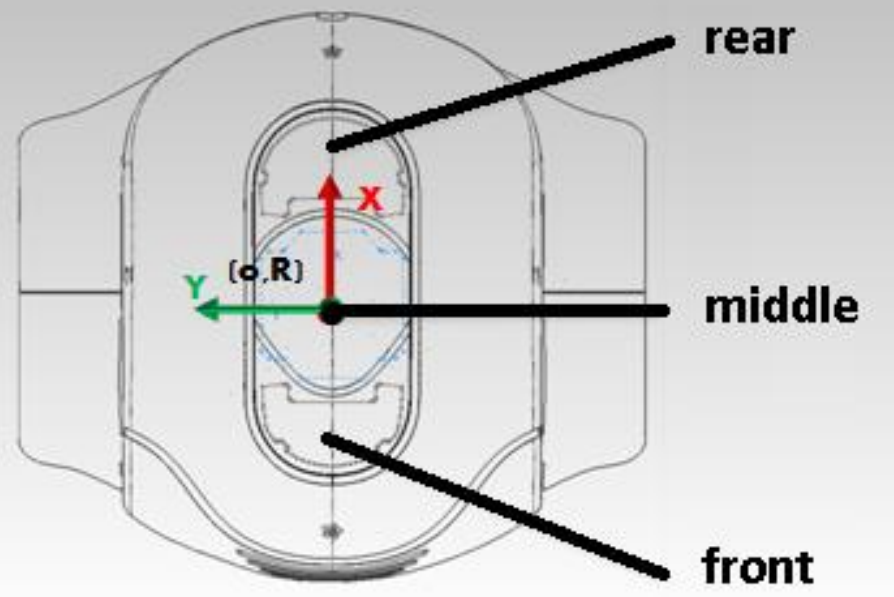

Abbildung 19 - drei taktile Kopfsensoren Quelle: Aldebaran Robotics, editiert vom Autor

Die taktilen Sensoren an der Hand sind wie folgt aufgegliedert. An jeder Hand befinden sich drei kapazitive Sensoren, zwei jeweils an den Seiten und einer auf dem Handrücken (siehe Abbildung 20). Die Bezeichner left, back und right sind so gewählt, dass die Zuordnung zu später verwendeten Methoden nachvollziehbar ist.
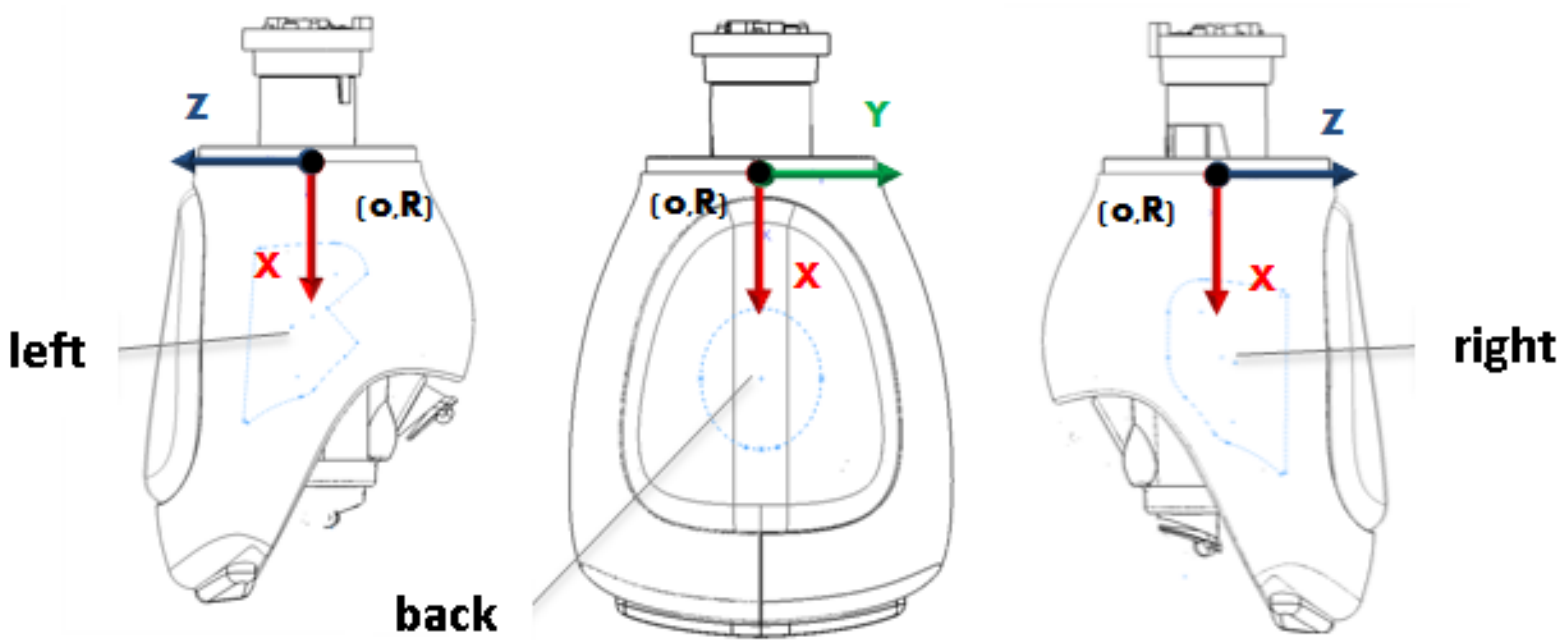

Abbildung 20 - taktile Handsensoren Quelle: Aldebaran Robotics, editiert vom Autor 


\subsubsection{LEDs}

Der NAO hat LEDs an den Ohren, auf dem Kopf (um die taktilen Sensoren herum), auf der Brust und an den Füßen. Um im Kontext dieser Arbeit zu bleiben, werden nachfolgend nur die LEDs um die Augen des NAOs beschrieben.

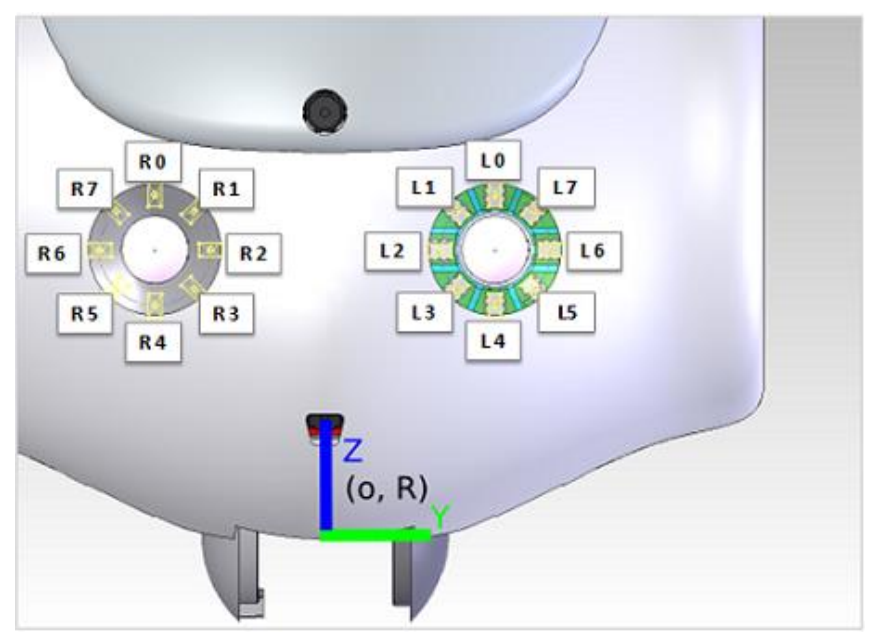

Abbildung 21 - LEDs an den Augen Quelle: Aldebaran Robotics

Die LEDs an den Augen können jeweils einzeln angesprochen werden. Damit ist es möglich, den NAO „zwinkern“ zu lassen oder durch die Augenfarbe eine bestimmte Stimmung zu suggerieren.

\subsubsection{Sprachsensorik}

Wie in Kapitel 3.4 beschrieben kann der NAO verbal kommunizieren. Dazu besitzt er zum einen vier im Kopf eingebaute Mikrofone (Abbildung 22).

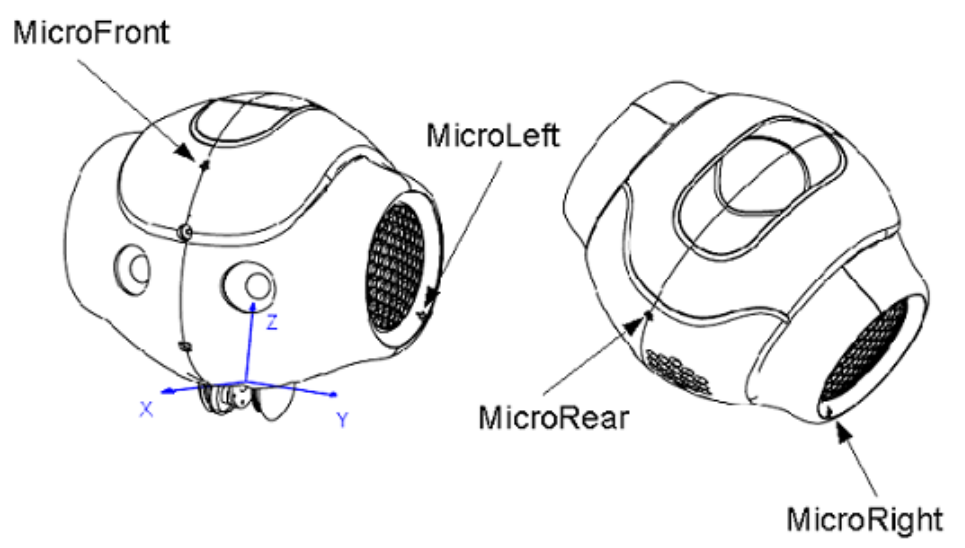

Abbildung 22 - Mikrofone des NAO-Roboters Quelle: Aldebaran Robotics

Diese ermöglichen es ihm, akustische Eingaben, Töne oder Sprache zu verstehen und zu verarbeiten. Durch die Anordnung der Mikrofone ist eine Ortung der Geräuschquelle möglich. 
Zum anderen verfügt er an den Kopfseiten über zwei Lautsprecher (Abbildung 23). Damit können akustische Ausgaben, Melodien und Sprache wiedergegeben werden. Die Lautstärke ist skalierbar.

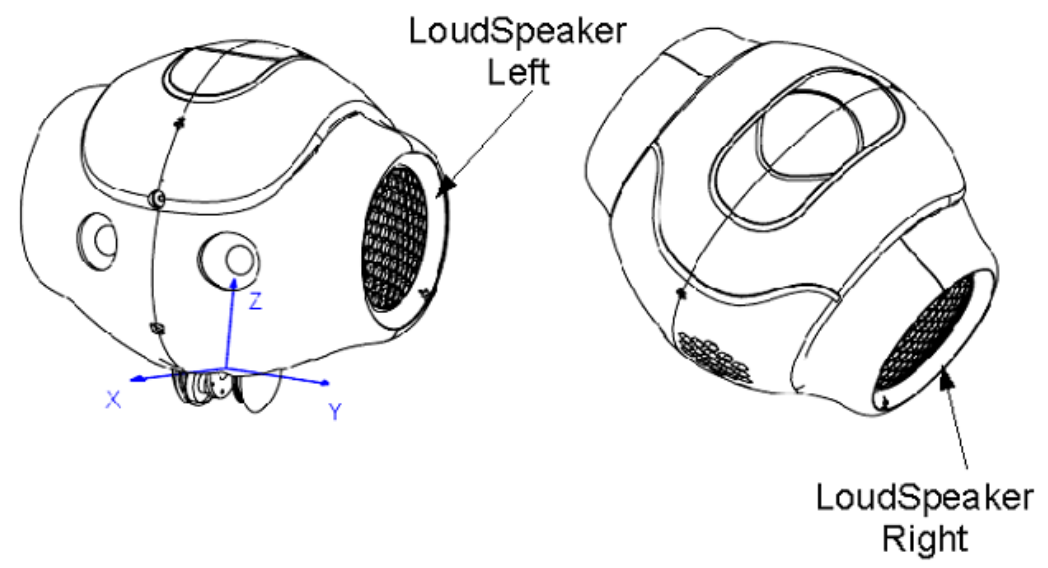

Abbildung 23 - Lautsprecher des NAO-Roboters Quelle: Aldebaran Robotics

\subsubsection{Kameras}

Mit den zwei am Kopf befindlichen Kameras bringt der NAO auch visuelle Fähigkeiten mit. Die Kameras ermöglichen eine Aufnahme von Videos mit einer Auflösung von 1288 x 968 Pixel* bei einer Pixelgröße von 1,9 $\mu$ m und einer Bildrate von 30 Frames pro Sekunde. Sie befinden sich ober- und unterhalb der Augen.

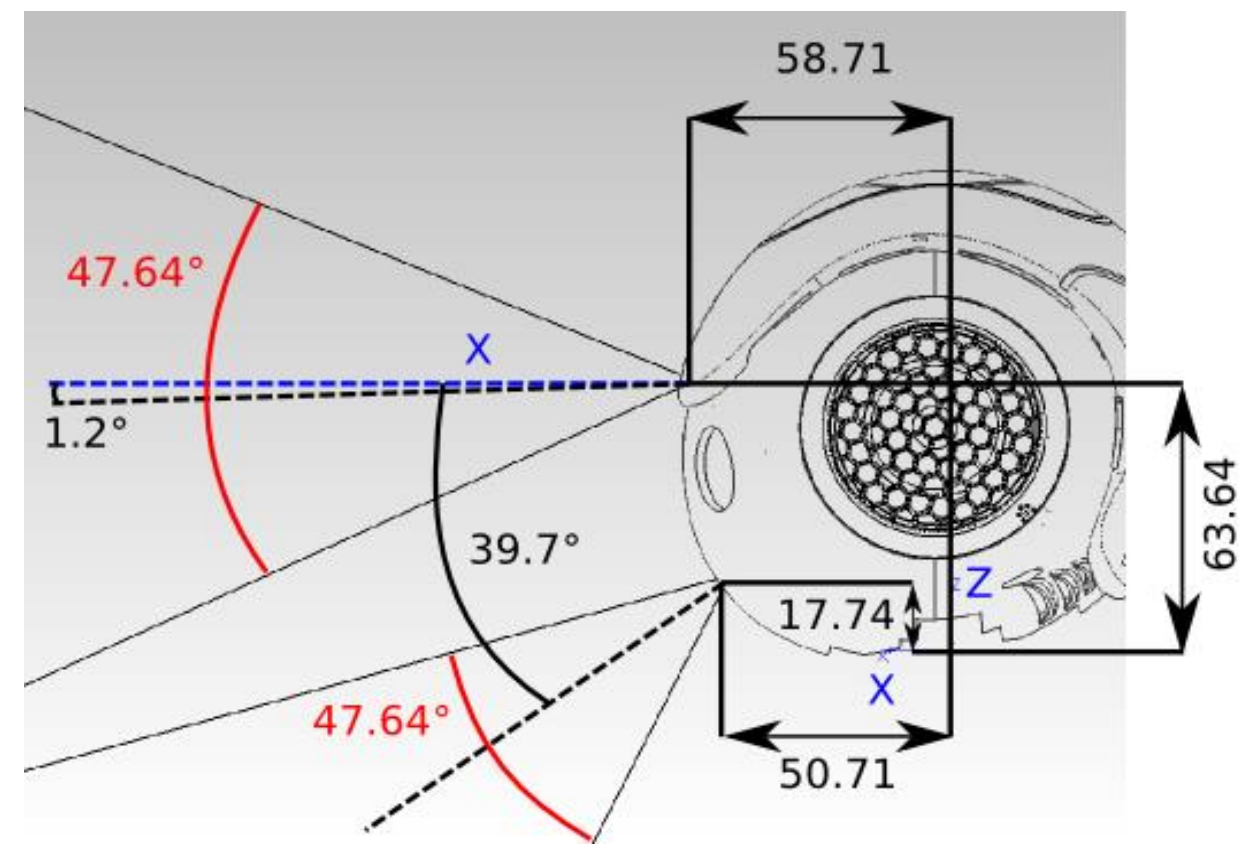

Abbildung 24 - Kameras am NAO Quelle: Aldebaran Robotics 


\subsubsection{Motorik und Bewegung}

Die in Kapitel 3.4 genannten 25 Freiheitsgrade des NAOs sind in Abbildung 25 zu sehen.

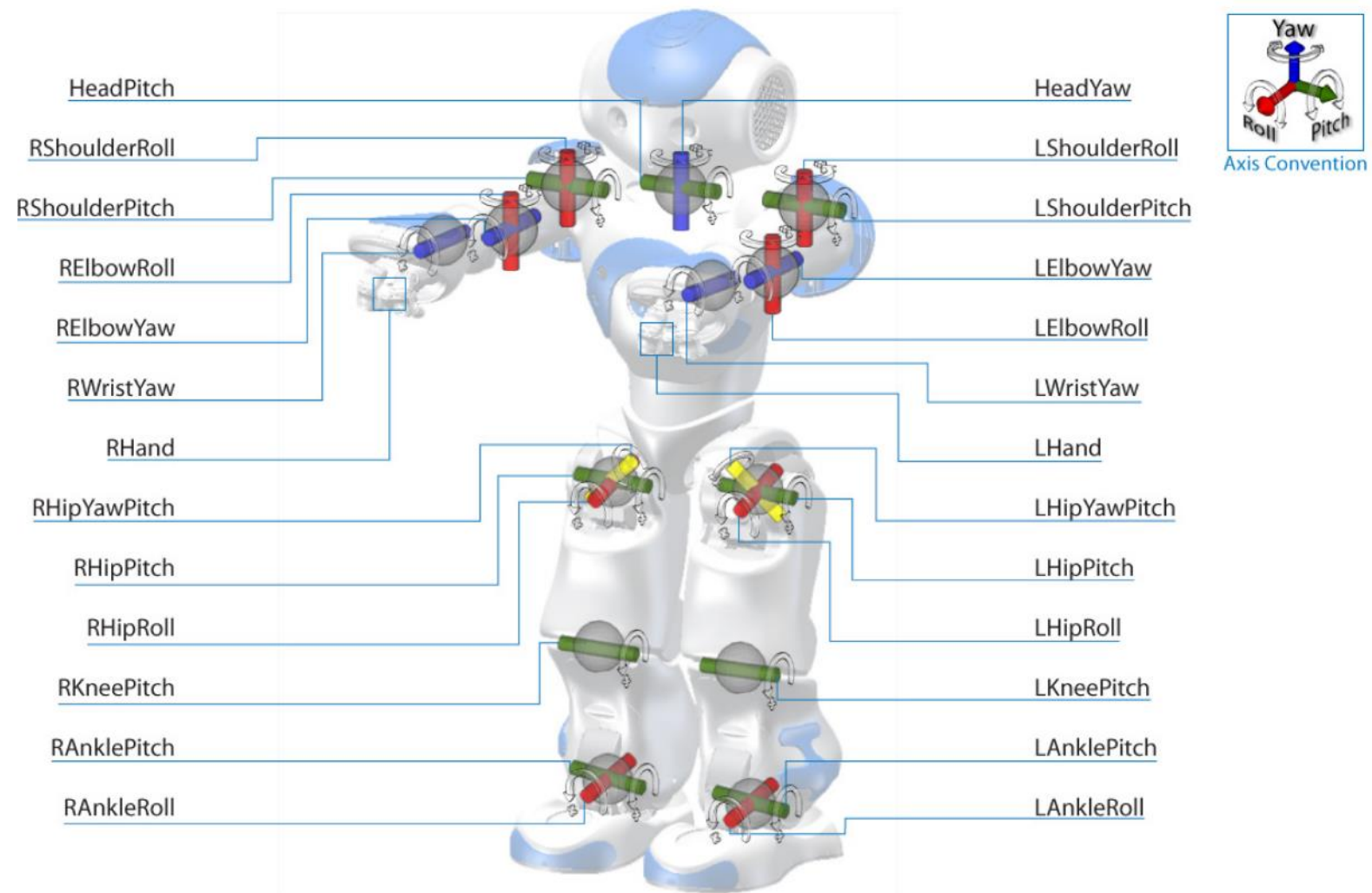

Abbildung 25 - Gelenke und Freiheitsgrade des NAO Quelle: Aldebaran Robotics

Die Freiheitsgrade werden durch insgesamt 25 Motorgelenke (engl. joints) ermöglicht. Aus der obigen Abbildung wird ersichtlich, welches Motorgelenk für welchen Teil des Roboterkörpers zuständig ist. Alle Gelenke einzeln zu beleuchten, brächte inhaltlich für das Konzeptverständnis keinen Mehrwert. Für eine detaillierte und Beschreibung wird an dieser Stelle auf die Dokumentation von Aldebaran Robotics verwiesen. ${ }^{18}$ Wie schon in Studie 4 in Kapitel 3.4 beschrieben, hat der NAO-Roboter einen sehr großen Bewegungsfreiraum, der es ihm ermöglicht, z.B. menschliche Gesten und Emotionen zu realisieren. Um die oben aufgelistete Hardware $\mathrm{zu}$ nutzen, stellt Aldebaran Robotics umfangreiche Entwicklungswerkzeuge zur Verfügung.

Bevor die Entwicklungswerkzeuge im übernächsten Kapitel 4.2 eingehend beleuchtet werden, wird im folgenden Kapitel 4.1.6 der Leap Motion Controller vorgestellt. Es handelt sich um eine zusätzliche Hardware, deren Einsatzmöglichkeiten bezüglich der zu entwickelnden Anwendung eruiert werden sollen.

\footnotetext{
${ }^{18}$ https://community.aldebaran.com/doc/1-14/family/nao_h25/joints_h25.html, Zugriff:24.08.2014
} 


\subsubsection{Der Leap Motion Controller}

Im Zuge der Themenfindung für diese Arbeit ergab sich die Idee, die in diesem Kapitel beschriebene Hardware mit einzubeziehen. Dabei liegt der Fokus nicht auf der programmiertechnischen Einbindung in das Projekt. Vielmehr soll ein Ansatz zur möglichen konzeptionellen Integration in das zu entwickelnde Anwendungsszenario aufgezeigt werden.

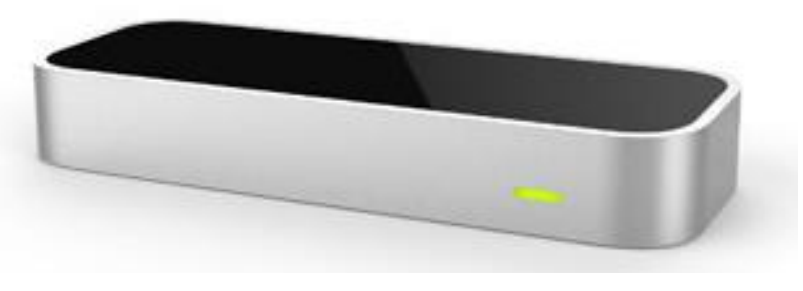

Abbildung 26 - der Leap Motion Controller Quelle: [46]

Der Controller (Abbildung 26) der Firma Leap Motion Ltd. ermöglicht eine berührungsfreie Interaktion mit dem PC. Mittels optischen Sensoren und Infrarotlicht erkennt er in einem Bereich von ca. $20 \mathrm{~cm}$ bis $60 \mathrm{~cm}$ über dem Controller Hände, einzelne Finger oder Eingabegeräte. Er ermöglicht das Erfassen und Nachverfolgen von Positionen, Gesten und Bewegungen der Objekte über dem Gerät [46]. Die Hände sollten sich innerhalb eines Radius` von ca. $50 \mathrm{~cm}$ um den Controller befinden, siehe Abbildung 27.

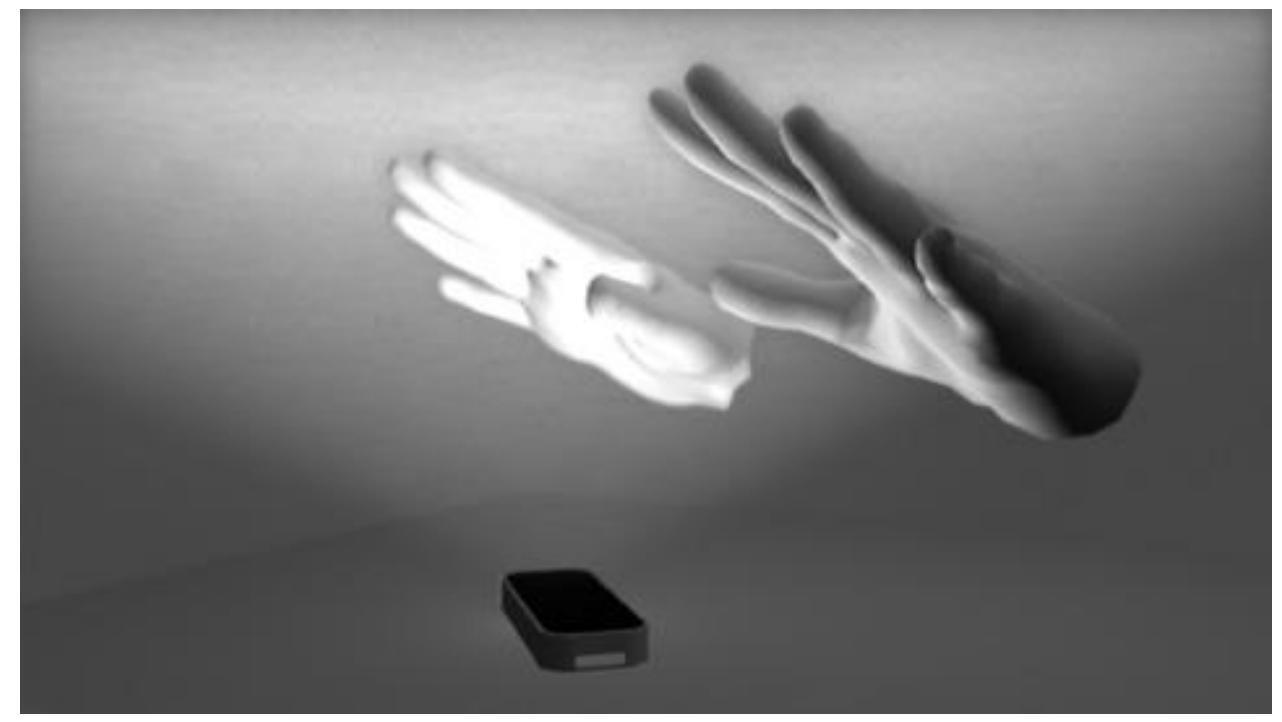

Abbildung 27 - Interaktionsraum über dem Controller, Quelle [46] 


\section{Architektur}

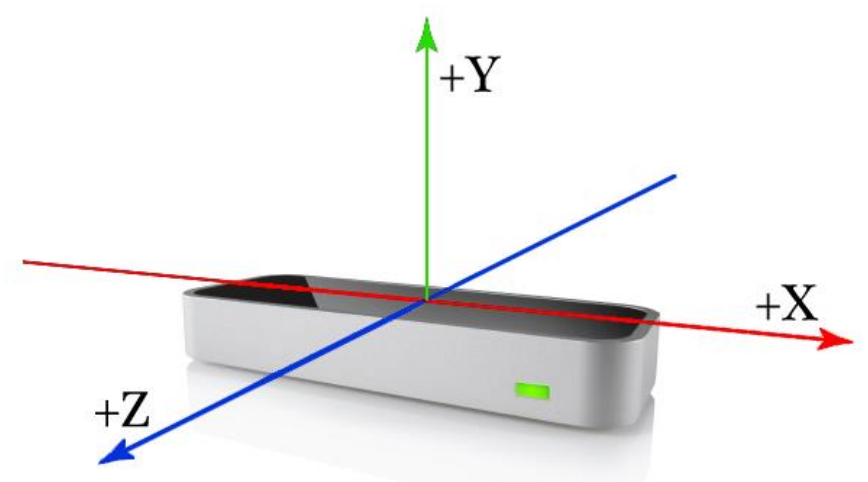

Abbildung 28 - Kartesisches Koordinatensystem des Leap-Motion Controllers Quelle: [23]

Der Interaktionsraum über dem Controller ist gleichzusetzen mit einem rechtshändigen Kartesischen Koordinatensystem*. Der Koordinatenursprung geht von der Mitte des Controllers aus. Die x und z-Achsen liegen in der horizontalen Ebene. Die y-Achse ist vertikal angeordnet und die Werte auf dieser Achse sind nur positiv. Die Werte auf der zAchse erhöhen sich positiv in Richtung des Benutzers (Abbildung 28).

\section{Programmierung}

Leap Motion stellt Bibliotheken für die Programmierung in $\mathrm{C}++, \mathrm{C} \#$, Python, Objective-C, Java und JavaScript bereit. Nach der Installation des Treibers steht dem Programmierer Hintergrundservice zur Verfügung. Der Controller generiert $200 \mathrm{Mal}$ pro Sekunde einen Snapshot vom Interaktionsraum und stellt die erfassten Objekte in einem Frame zur Verfügung. Dieses Frame ist der Ausgangspunkt für alle Zugriffe auf die Objekte. Es ist die Wurzel des gesamten Datenmodells. Abbildung 31 zeigt

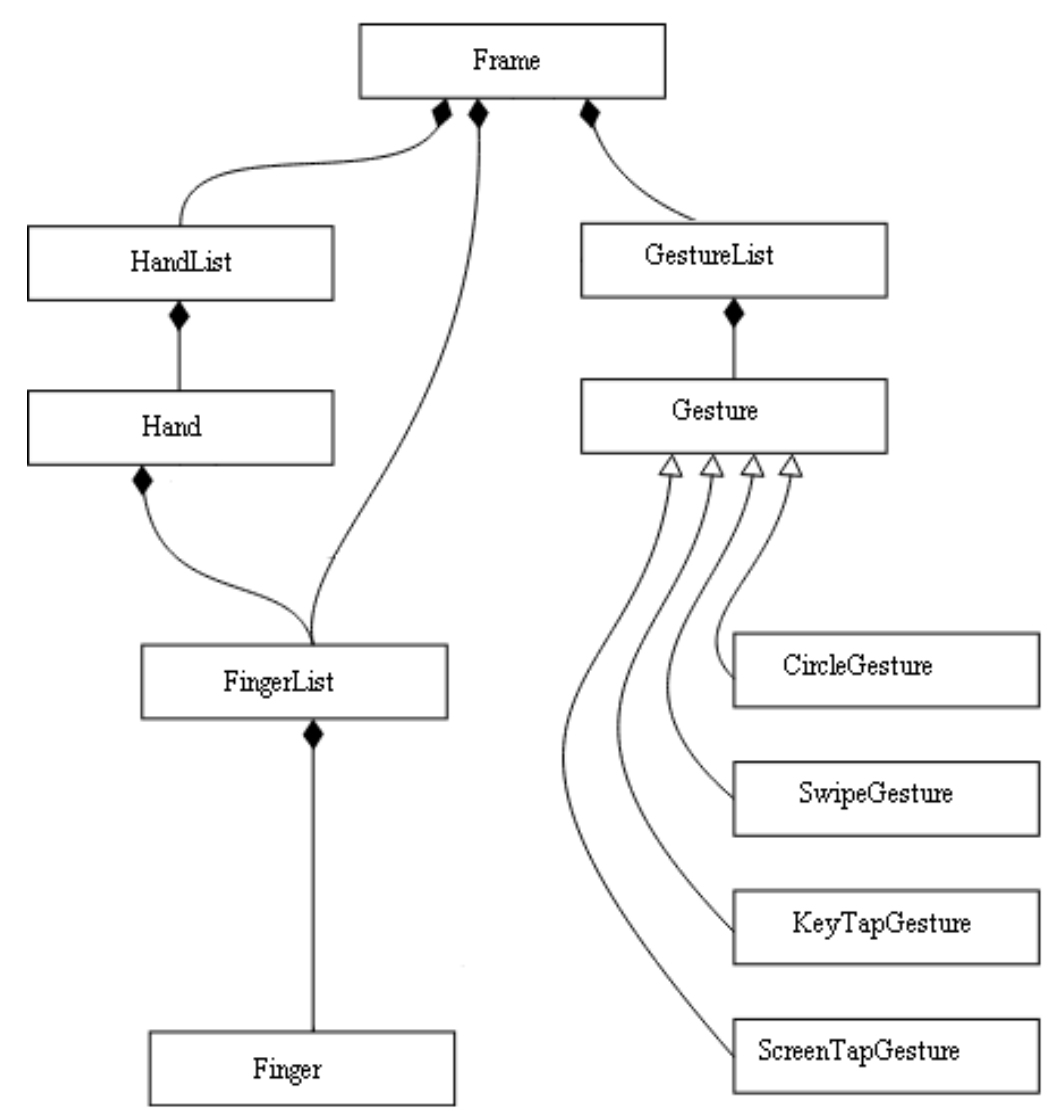
Abbildung 31 - Trackingdaten die vom Controller geliefert werden in vereinfachter Form die bereitgestellten Trackingdaten. 
Wie rechts unten in Abbildung 31zu sehen ist, werden bibliotheksübergreifend unter anderem vier Callback-Methoden bereitgestellt, mit denen gezielt Handgesten über dem Controller erkannt werden. In Tabelle 1 sind diese abgebildet und erläutert.

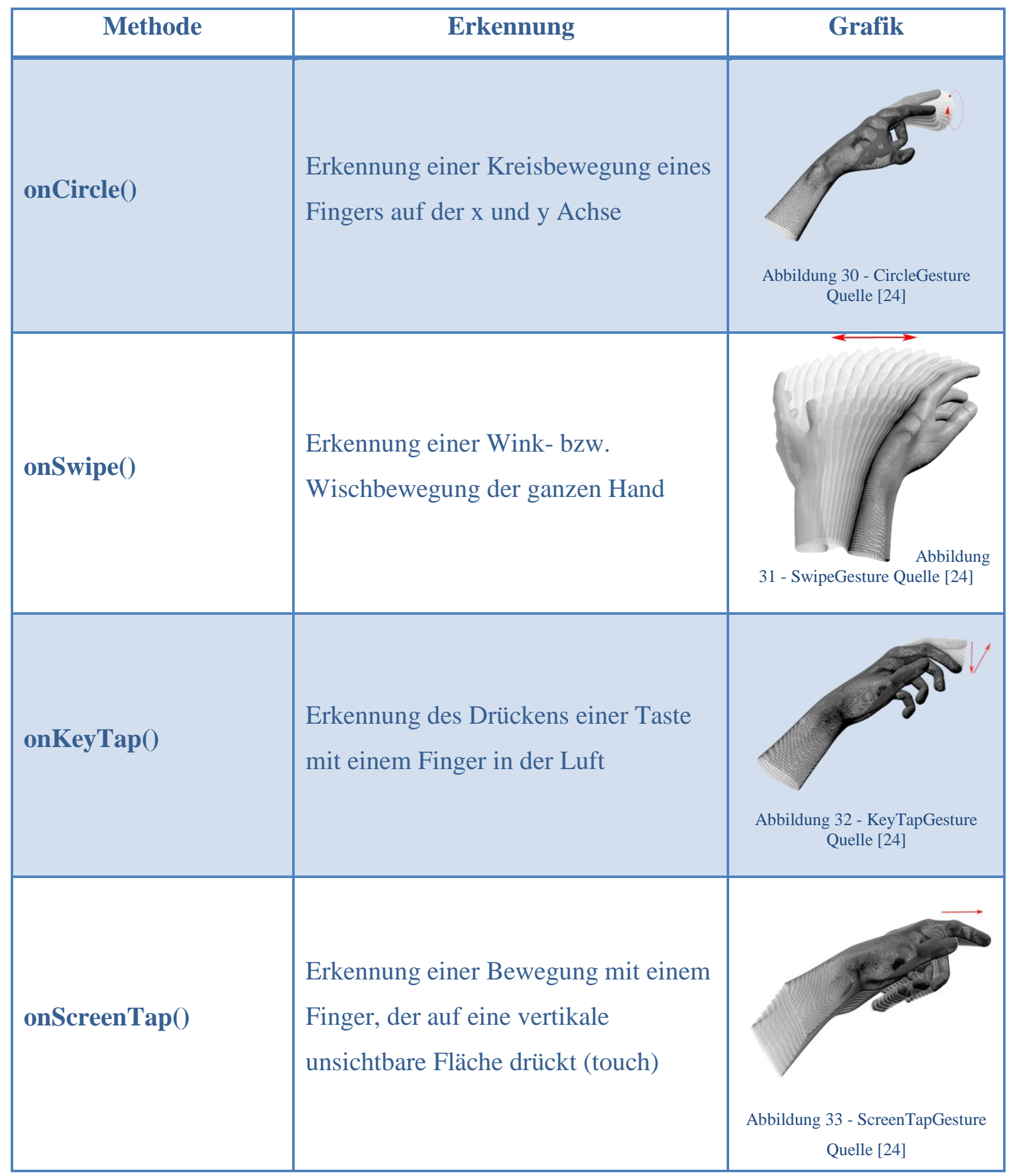

Tabelle 1 - vordefinierte Handgesten

Eine weitere nutzbringende Eigenschaft ist die Möglichkeit, einzelne Hände oder Finger direkt anzusprechen und deren Position zu ermitteln. Weiterhin kann somit auch die Anzahl der Hände und Finger innerhalb des Frames erfasst und bestimmt werden. Die geografische 
Position der Hände und Fingerspitzen lässt sich ebenfalls erfassen. In Abbildung 34 wird beispielhaft der Zugriff auf die Position der Fingerspitze oder die Handposition gezeigt.

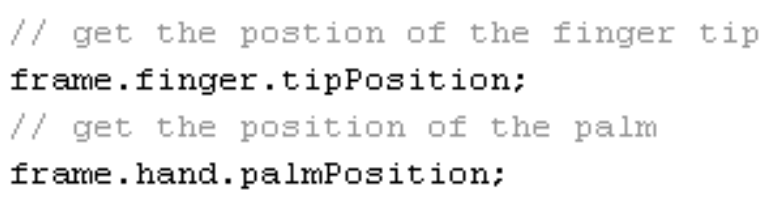

Abbildung 34 - Zugriff und Erfassung der Fingerspitzen Quelle: [23]

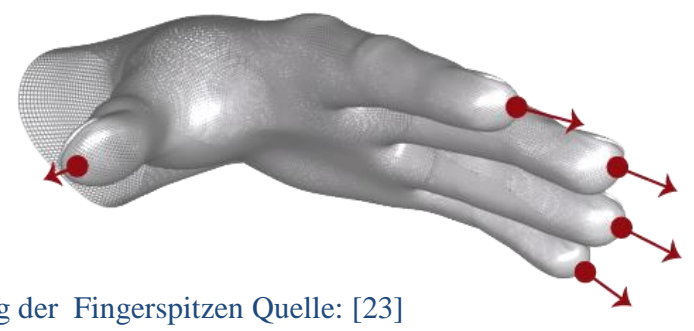

Zusammenfassend ist zu sagen, dass mittels des Leap-Motion Controllers eine Interaktion mit den Händen oder Fingern innerhalb einer Anwendung sinnvoll sein kann. Auf der DeveloperWebsite des Leap Motion Projektes findet sich eine ausführliche Referenz aller weiteren Methoden. ${ }^{19}$ Wie und ob der Controller in die zu entwickelnde Anwendung eingebunden werden kann, wird im Konzept nochmals aufgegriffen. Zunächst werden die verschiedenen Entwicklungsumgebungen vorgestellt.

\footnotetext{
${ }^{19}$ https://developer.leapmotion.com/documentation/skeletal/csharp/devguide/Leap_Tracking.html, Zugriff 31.07.2014
} 


\subsection{Entwicklungssoftware}

Vorrausetzung für die Verwendung der Entwicklungswerkzeuge ist das Verständnis der Softwarearchitektur des Roboters. Auf dem NAO-Roboter läuft ein embedded x86 LinuxBetriebssystem. Das unter der GNU*-Lizenz stehende Gentoo-Derivat nennt sich „OpenNAO“. Auf dem Betriebssystem wird das NAOqi-Framework* als Middleware* eingesetzt. Das NAOqi-Framework wurde von Aldebaran Robotics bereitgestellt, um den NAO-Roboter zu programmieren, kontrollieren und zu steuern. Das Framework bietet ein Event-, Ressourcen-, Synchronisierungs- sowie Parallelisierungsmanagement und wird damit allen Ansprüchen an die Roboterprogrammierung gerecht. Eine ausführliche Betrachtung folgt im nächsten Abschnitt. Es dient ebenfalls der Erstellung und Ausführung von Behaviors (Bewegungsmustern) und Modulen. Ein Behavior ist eine Zusammenstellung von Instruktionen, die an den Roboter gesendet oder auf ihm installiert werden können. Beispiele für solche Bewegungsmuster sind unter anderem die Anweisung zu laufen, zu sprechen, zu tanzen oder eine Sounddatei abzuspielen.

\section{NAOqi}

Der Hersteller stellt eine API (Programmierschnittstelle) mit umfangreichen Modulbibliotheken zur Verfügung, die über die Middleware NAOqi mit diversen Programmiersprachen und auf verschiedene Weise angesprochen, genutzt und erweitert werden kann. Um die Ausführung der Module auf dem NAO zu verstehen, ist es nötig, das NAOqi-Framework näher zu betrachten.

Das NAOqi-Framework verwaltet alle Module. Module sind Sammlungen von Funktionen. Mehrere Module bilden eine Modulbibliothek. Ein Beispiel ist das bereitgestellte ALTextToSpeech-Modul. Es bietet Methoden an, die einen geschriebenen Text in einer bestimmten Sprache über die Lautsprecher des NAO-Roboters akustisch ausgeben. Es existiert eine Vielzahl solcher Module. Diese sind in die Kategorien Core, Motion, Audio, Vision, Sensors Tracker und DCM unterteilt. Eine vollständige Übersicht aller Module zeigt Tabelle 2.

\begin{tabular}{|l|}
\hline Core \\
\hline ALBehaviorManager \\
\hline ALConnectionManager \\
\hline ALExtractor \\
\hline ALMemory \\
\hline ALModule \\
\hline
\end{tabular}

\begin{tabular}{|l|}
\hline Vision \\
\hline ALBacklightingDetection \\
\hline ALDarknessDetection \\
\hline ALFaceDetection \\
\hline ALLandmarkDetection \\
\hline ALMovementDetection \\
\hline
\end{tabular}




\begin{tabular}{|l|} 
ALPreferences \\
\hline ALResourceManager \\
\hline ALVisionExtractors \\
\hline Audio \\
\hline ALAudioDevice \\
\hline ALAudioPlayer \\
\hline ALAudioRecode \\
\hline ALAudioSourceLocalisation \\
\hline ALSoundDetection \\
\hline ALSpeechRecognition \\
\hline ALTextToSpeech \\
\hline
\end{tabular}

\begin{tabular}{|l|}
\hline ALSonar \\
\hline ALLeds \\
\hline
\end{tabular}

\begin{tabular}{|l|}
\hline Tracker \\
\hline ALFaceTracker \\
\hline ALRedBallTracker \\
\hline
\end{tabular}

\begin{tabular}{|l|} 
ALPhotoCapture \\
\hline ALRedBallDetection \\
\hline ALVideoDevice \\
\hline ALVideoRecorder \\
\hline ALVisionRecognition \\
\hline ALVisualCompass \\
\hline
\end{tabular}

\begin{tabular}{|l|}
\hline DCM \\
\hline DCM \\
\hline
\end{tabular}

\begin{tabular}{|l|}
\hline Motion \\
\hline ALMotion \\
\hline ALNavigation \\
\hline ALRobotPosture \\
\hline
\end{tabular}

Tabelle 2 - Modulübersicht im NAOqi-Framework

In der Konzeptentwicklung wird die obige Tabelle nochmals aufgegriffen und auf die Verwendung einzelner Module speziell eingegangen.

Die ausführende und übergeordnete Instanz des Frameworks ist der Broker*. Dem Broker sind alle an ihm angemeldeten Module und Bibliotheken (engl. libraries) bekannt. Er ist für das Laden der Module verantwortlich und stellt den Verzeichnisdienst zum Auffinden selbiger zur Verfügung. Zusätzlich steuert er den externen Netzwerkzugriff auf die Methoden der Module. In Abbildung 35 wird die Architektur deutlich. Der Broker liest eine autoload.ini Datei beim Start des Roboters aus und lädt die zu den Bibliotheken gehörigen Module.

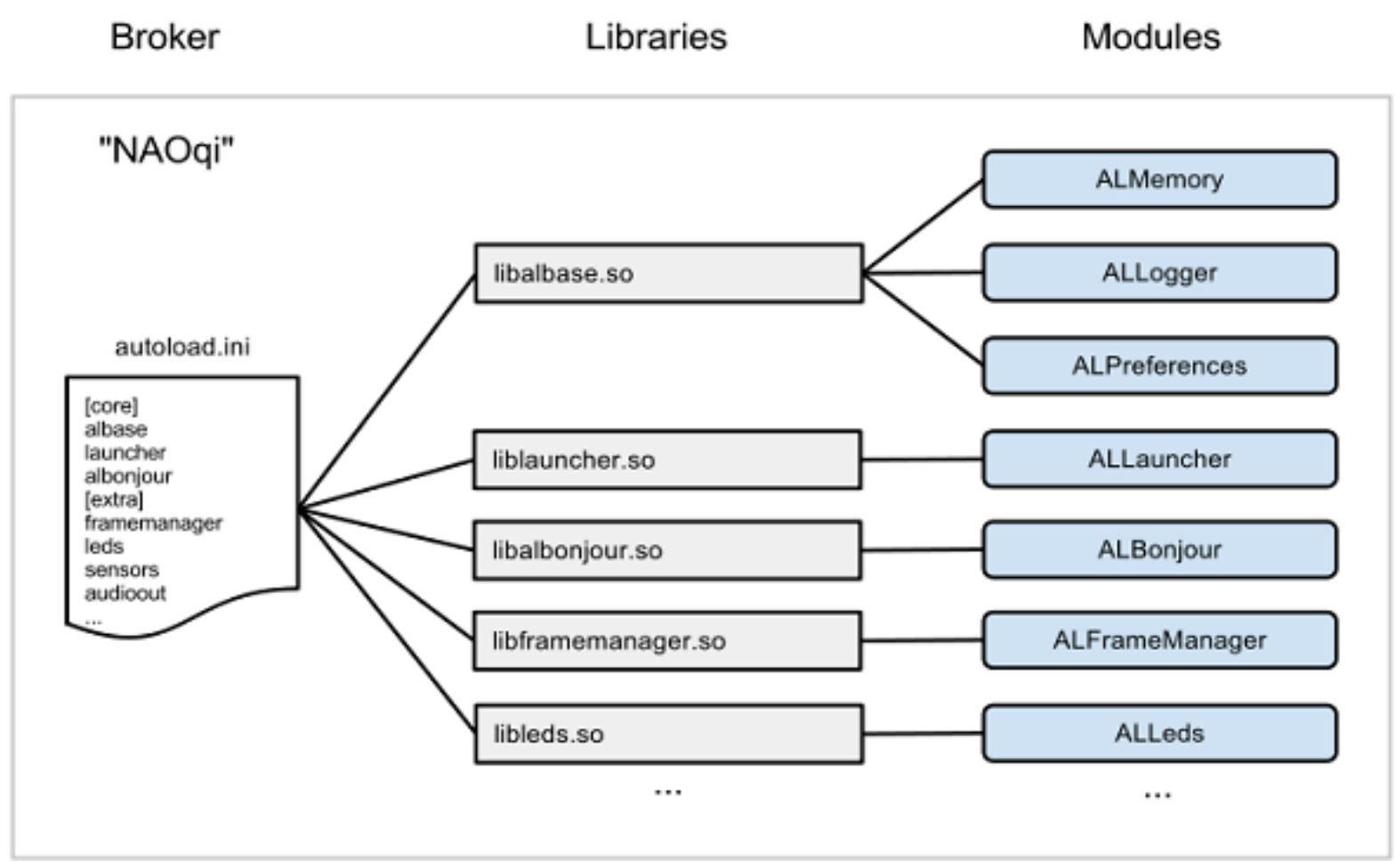


„Durch das Laden von Modulen wird eine Hierarchie aufgespannt, welche den Broker als Wurzelknoten mit den einzelnen Modulen verbindet und diese wiederum mit den eigenen Methoden assoziiert" [47]. Abbildung 36 visualisiert diese Aussage zusätzlich. Der Broker kennt nach dem Start alle Methoden und stellt diese auf Wunsch bereit. Sobald eine Bibliothek vom Broker geladen wird, wird das Modul, welches auch als Klasse ${ }^{20}$ angesehen wird, instanziiert.

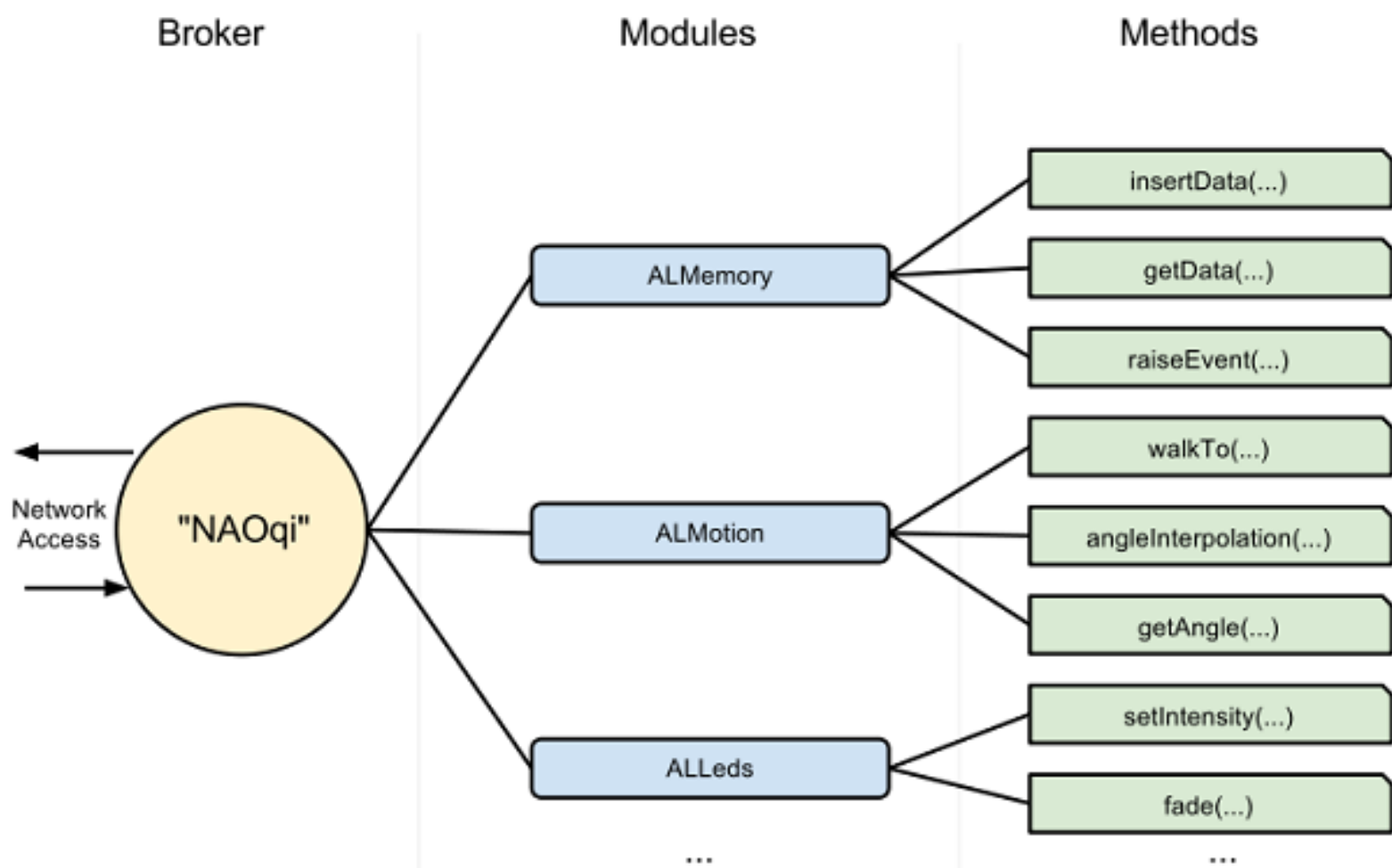

Abbildung 36 - Zusammenhang: Broker, Module und Methoden Quelle: Aldebaran Robotics

\section{Programmierung}

Die Programmierung kann in drei Szenarien eingeteilt werden:

1. Es können in C++ und Python programmierte Module* auf einem Linux-Betriebssystem mit einem Cross-Compiler ${ }^{21}$ kompiliert und diese dann lokal auf dem NAO ausgeführt werden. Diese embedded Programmierung erlaubt eine autonome Ausführung von Modulen auf dem Roboter.

${ }^{20}$ Das Wort „Klasse“ entspricht hier dem Verständnis, wie es in der objektorientierten Programmierung verwendet wird.

21 Unter einem Cross-Compiler versteht man einen Compiler, der auf einem Entwicklersystem läuft, aber kompatible Objektdateien oder ausführbare Programme in Maschinensprache für andere Systeme erzeugt. 
2. Mit den Programmiersprachen $\mathrm{C}++$, Python, Java, Matlab*, Ubi* und NET* kann Quellcode erzeugt werden, mit dem der Roboter per remote gesteuert werden kann. Das heißt, auf einem PC mit dem Betriebssystem Windows /MAC OSX oder Linux erstellter Quellcode wird über Netzwerk (WLAN/Ethernet) auf dem NAO-Roboter ausgeführt.

3. Die dritte Variante, wie der Roboter programmiert werden kann, ist die Verwendung der Desktop-Software Choregraphe Suite. Mit ihr kann man auf die gesamte NAOqi-Api zugreifen und mit, auf der Programmiersprache Python basierenden, sogenannten Boxen arbeiten. Diese Boxen sind gleichzusetzen mit den oben genannten Modulen.

Zur Veranschaulichung hilft es, folgende Abbildung zu betrachten:

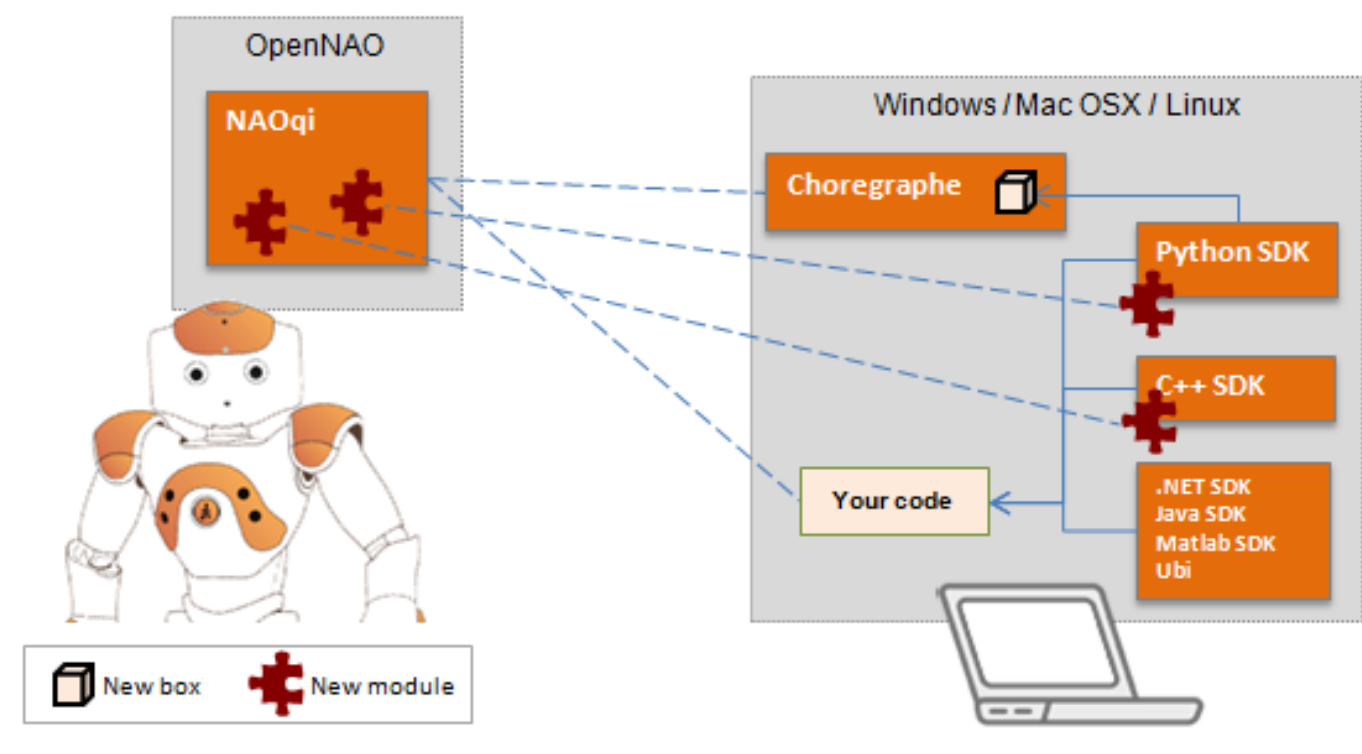

Abbildung 37 - Übersicht der Programmiertools Quelle: Aldebaran Robotics

In Abbildung 37 sieht man sehr gut die Gleichstellung von Boxen und Modulen. Ein Behavior lässt sich aus verschiedenen Modulen/Boxen zusammensetzen. Die Puzzleteile repräsentieren lokal ausführbare Module. Die gestrichelten Linien zeigen die Ausführbarkeit des Quellcodes per remote.

Das NAOqi Framework kann remote oder lokal ausgeführte Module gleichzeitig verwenden. Erreicht wird diese Transparenz durch Proxys, die für den Programmierer den Einsprungspunkt zur Modelnutzung darstellen. Der Proxy meldet sich über das Modul am Broker an und bekommt ein Objekt mit allen Methoden des Moduls zur Verfügung gestellt. 


\section{Auswahl der Entwicklungsumgebung}

Im folgenden Abschnitt werden die drei Entwicklungsumgebungen

- $\quad$ Choregraphe Suite 1.14.5

- $\quad \mathrm{SDK}$ für $\mathrm{C}++$

- SDK für Java

vorgestellt. Anhand der in

Tabelle 3 aufgelisteten Kriterien werden diese bewertet. Nach diesen Kriterien wird vom Autor ein geeignetes Framework für die Umsetzung der Anwendung ausgewählt.

\begin{tabular}{|c|c|}
\hline Kriterium & Beschreibung \\
\hline lokal ausführbar & $\begin{array}{l}\text { Der Quellcode kann auf dem Entwicklersystem cross- } \\
\text { kompiliert und direkt auf dem NAO autonom ausgeführt } \\
\text { werden. }\end{array}$ \\
\hline remote ausführbar & $\begin{array}{l}\text { Der Quellcode oder erstellte Behaviors können remote } \\
\text { über das Netzwerk auf dem NAO ausgeführt werden. }\end{array}$ \\
\hline Bewegungen entwickeln & $\begin{array}{l}\text { Die Implementierung und Entwicklung von } \\
\text { Bewegungsabläufen wird durch das Framework visuell } \\
\text { unterstützt. }\end{array}$ \\
\hline Ausführungsgeschwindigkeit & $\begin{array}{l}\text { Die Ausführungsgeschwindigkeit der Behaviors oder des } \\
\text { Quellcodes ist zumutbar zügig. }\end{array}$ \\
\hline Eventhandling & $\begin{array}{l}\text { Die Entwicklungsumgebung bietet die Möglichkeit, sich } \\
\text { an Events anzumelden, und wird bei Eintreten dieser über } \\
\text { Callback -Methoden informiert. }\end{array}$ \\
\hline $\begin{array}{l}\text { Betriebssystem } \\
\text { (Linux/MAC OSX) }\end{array}$ & $\begin{array}{l}\text { Die Entwicklungsplattform ist unter Linux oder MAC } \\
\text { OSX lauffähig. }\end{array}$ \\
\hline Betriebssystem (Windows) & $\begin{array}{l}\text { Die Entwicklungsplattform ist unter Microsoft Windows } \\
\text { lauffähig. }\end{array}$ \\
\hline $\begin{array}{l}\text { optimiert für eingebettete } \\
\text { Systeme }\end{array}$ & $\begin{array}{l}\text { Die Entwicklungsumgebung ist optimiert für eine } \\
\text { hardwarenahe Programmierung. }\end{array}$ \\
\hline
\end{tabular}




\subsubsection{Choregraphe Suite}

Die multi-Plattform-Desktopanwendung Choregraphe Suite 1.14 .5 ist eine grafische Entwicklungsumgebung. Mit ihr ist es auf einfache Weise möglich, auf die in der NAOqi-API bereitgestellten Module zuzugreifen und diese $\mathrm{zu}$ benutzen. Eigene Animationen und Behaviors können damit erstellt werden, ohne Quellcodekenntnisse zu schreiben. Die Entwicklungsumgebung wird für die Betriebssysteme Windows, Linux und MAC OSX bereitgestellt. Sie bietet Funktionen, um den NAO-Roboter zu kontrollieren und die Hardware $\mathrm{zu}$ überwachen. Außerdem kann die Software einen NAO-Roboter emulieren und erstellte Behaviors auf jenem virtuellen Roboter ausführen. In Choregraphe lassen sich sogenannte vordefinierte „Boxen“ verwenden und zu komplexen Programmen zusammenfügen. Diese Boxen repräsentieren alle wichtigen Funktionen, die die NAOqi-API bereitstellt. Es ist möglich, eigenen in Python geschriebenen Quellcode in Choregraphe zu integrieren.

Abbildung 38 zeigt eine Übersicht der Benutzeroberfläche:

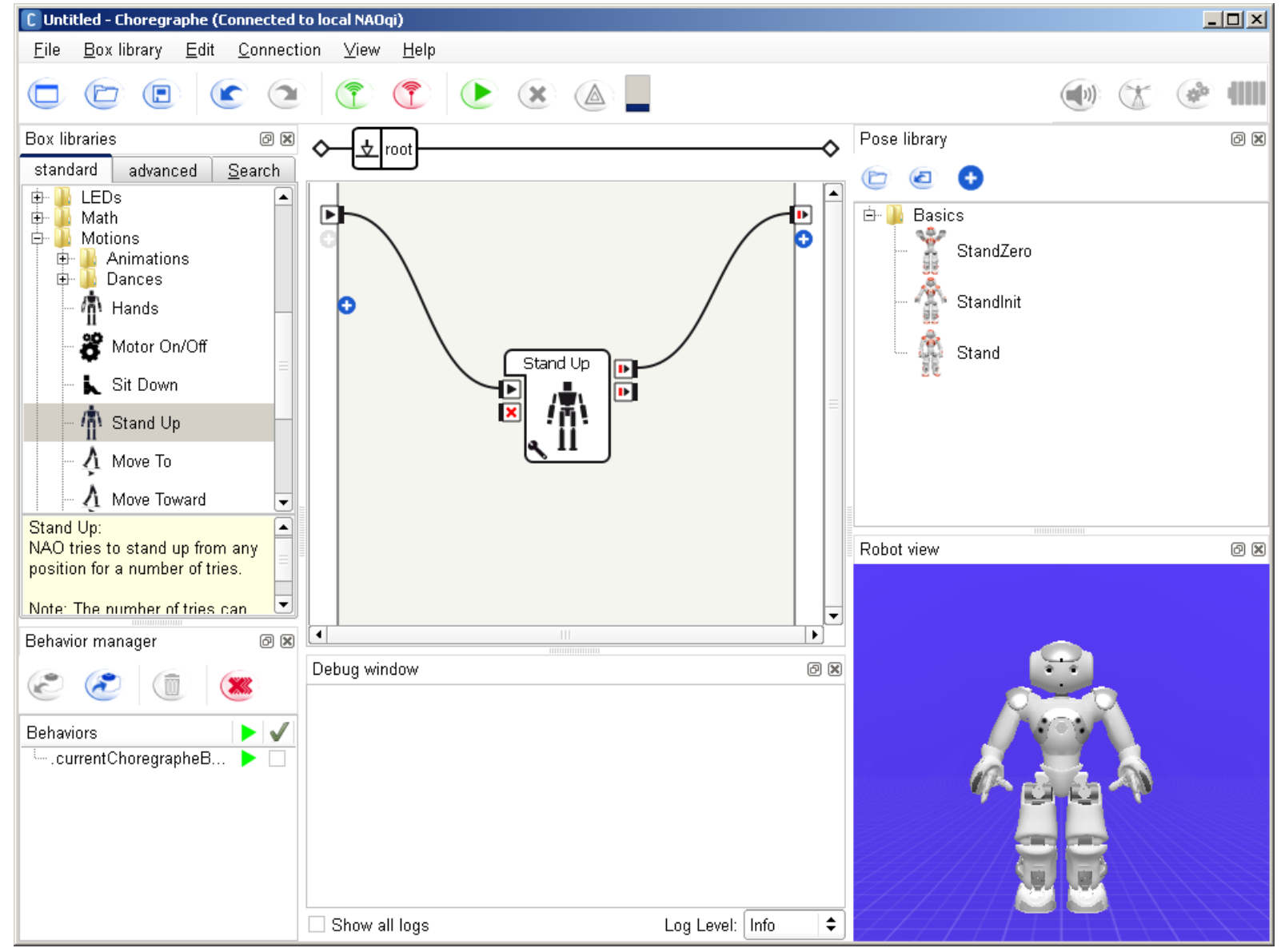

Abbildung 38 - Choregraphe Suite

Zentral ist eine sogenannte Box zu sehen. Diese Stand $U p$-Box lässt, wenn sie ausgeführt wird, den NAO aufstehen. Der Bereich, in dem sie sich befindet, heißt flow diagram. In der 
unteren rechten Ecke befindet sich die robot view. Über sie lassen sich die Bewegungen des Roboters visuell nachverfolgen. Auf der linken Seite befindet sich eine Übersicht aller vordefinierten Behaviors und Funktionen. Links unten werden die auf dem NAO-Roboter installierten Behaviors aufgelistet. Rechts über der robot view ist die pose libray zu sehen. Sie enthält drei Standardposen des Roboters.

Der Programmablauf wird in Flussdiagrammmanier entwickelt. Um den NAO-Roboter eine Aktion ausführen zu lassen, zieht man per Drag and Drop von der linken Seite eine gewünschte Box in das flow diagram und verbindet diese mit dem Startknoten oben links im flow diagram.

Wenn der Benutzer, um eine Behavior auszuführen, auf den grünen Pfeil klickt, wird per remote die NAOqi des verbundenen Roboters angesprochen und die Anweisung über das Netzwerk ausgeführt. Das kann sich unter Umständen nachteilig auf die Ausführungsgeschwindigkeit der Programme zu auswirken. Die Zeit zwischen dem Drücken des grünen Pfeils und der ersten Bewegung des NAO-Roboter ist abhängig von der Menge der zu übertragenen Daten und der Qualität der Netzwerkverbindung.

\section{Animationen}

Eine für die Umsetzung wichtige Funktion der Software ist der Animationsmodus. Mit ihm lassen sich sehr komfortabel neue Bewegungen (Motions) und Posen für den Roboter programmieren. Dabei wird der Roboter wie eine Puppe in die gewünschte Position bewegt. Die Sensoren an den Gelenken geben exakt die Winkelpositionen und Lagerichtung der Motoren an die Software zurück. Der Zustand wird gespeichert und kann als neues Behavior in die Bibliothek aufgenommen und weiterverarbeitet werden. Sinnvoll ist an dieser Stelle zu erwähnen, dass die aufgenommenen Werte und Bewegungen in C++ oder Python Quellcode exportiert werden können. Im Anhang A1 wird die Vorgehensweise, eine Animation zu erstellen, detailliert beschrieben.

\subsubsection{C++ Environment}

Das C++ Framework ist die einzige Entwicklungsumgebung, der der komplette Funktions-, und Modulumfang der NAOqi API zur Verfügung steht. Mit C++ lassen sich EchtzeitAnwendungen programmieren, die mit höchstmöglicher Geschwindigkeit auf dem Roboter ausgeführt werden können. Für eine unkomplizierte Kompilierung des Quellcodes empfiehlt Aldebaran Robotics die Verwendung von CMake* mit dem qiBuild* Framework. 
Für die genaue Erstellung eines lokalen Moduls in C++ wird an dieser Stelle auf das Tutorial von Georg Labbé, welches auf der DVD-Beilage zu finden ist, verwiesen. Im Rahmen der Lehrveranstaltung „Embedded C++“ des 6. Semesters des Studiengangs Telematik an der TH Wildau wurde eine virtuelle Maschine (VM) bereitgestellt, welche als vollständig vorkonfigurierte Entwicklungsumgebung für C++ Programme dient. Basierend auf der LinuxDistribution Ubuntu 12.04 ist der Compiler des qiBuild Frameworks, die Desktopsoftware Choregraphe und die IDE* QtCreator* installiert und vorkonfiguriert.

Mit C++ ist es möglich, sich an Events zu registrieren und entsprechende Callback-Methoden auszuwerten. Folgende Zusammenstellung zeigt die wichtigsten Events, die die NAOqi-API bereitstellt. Sie wurde vom Autor der Übersicht halber gekürzt, ohne essentielle Eventmethoden wegzulassen. Einzelne Events aus Tabelle 4 werden in der Umsetzung nochmals herangezogen und näher betrachtet.

\begin{tabular}{|l|}
\hline Sensors \\
\hline ChestButtonPressed \\
\hline DoubleClickOccured \\
\hline FrontTactilTouched \\
\hline HandLeftBackTouched \\
\hline HandLeftLeftTouched \\
\hline HandLeftRightTouched \\
\hline HandRightBackTouched \\
\hline HandRightLeftTouched \\
\hline HandRightRightTouched \\
\hline LeftBumperPressed \\
\hline MiddleTactilTouched \\
\hline RearTactilTouched \\
\hline RightBumperPressed \\
\hline SimpleClickOccured \\
\hline TripleClickOccured \\
\hline SonarLeftDetected \\
\hline SonarLeftNothingDetected \\
\hline SonarRightDetected \\
\hline SonarRightNothingDetected \\
\hline
\end{tabular}

\begin{tabular}{|l|}
\hline Audio \\
\hline CurrentWord \\
\hline LastWordRecognized \\
\hline SpeechDetected \\
\hline TextDone \\
\hline TextStarted \\
\hline WordRecognized \\
\hline SoundDetected \\
\hline SoundLocated \\
\hline PositionOfCurrentWord \\
\hline
\end{tabular}

\begin{tabular}{|l|}
\hline Core \\
\hline BatteryChargeChanged \\
\hline BehaviorsRun \\
\hline BodyStiffnessChanged \\
\hline HotJointDetected \\
\hline NAOqiReady \\
\hline NetworkStateChanged \\
\hline robotHasFallen \\
\hline robotPoseChanged \\
\hline
\end{tabular}

\begin{tabular}{|l|}
\hline Vision \\
\hline BacklightingDetected \\
\hline DangerousObstacleDetected \\
\hline DarknessDetected \\
\hline FaceDetected \\
\hline MovementDetected \\
\hline PictureDetected \\
\hline redBallDetected \\
\hline LandmarkDetected \\
\hline
\end{tabular}




\subsubsection{Java Environment}

Für die Programmierung von Modulen in Java ist es notwendig, die JNAOqi*-Bibliothek einzubinden. Mit ihr ist es möglich, auf die von der NAOqi bereitgestellten Module auf dem NAO zuzugreifen. Dies funktioniert nur per Fernsteuerung, da auf dem NAO keine JVM* installiert ist. Der Quellcode wird auf dem Rechner ausgeführt und steuert durch entsprechende NAOqi-Aufrufe über den Proxy den NAO fern. Die bereitgestellte Java-API ist wie ein Wrapper um die NAOqi herum zu verstehen. Die Syntax des Modulaufrufs ist dieselbe wie in Python oder .Net.

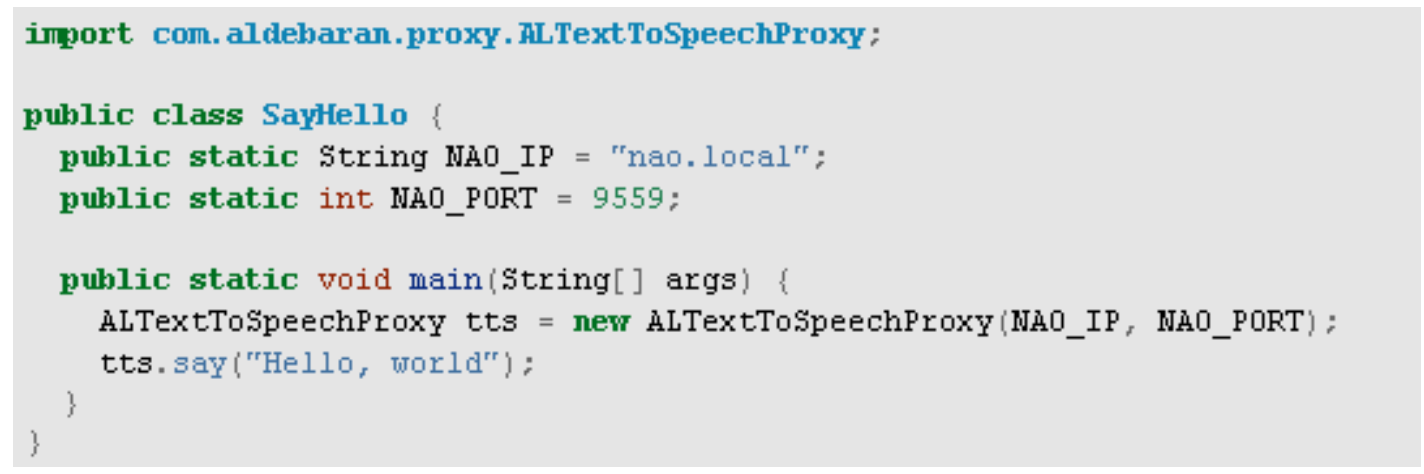

Abbildung 39 - Java Syntax für einen Modulaufruf Quelle: Aldebaran Robotics

Abbildung 40 zeigt die Erstellung der Klasse SayHello. Sie besitzt die Klassenattribute NAO_IP und NAO_PORT. Die erste Anweisung in der main-Methode erstellt einen TextToSpeechProxy und übergibt die beiden Klassenattribute als Parameter. Die Anweisung tts.say ( ) lässt den NAO-Roboter die Worte „Hello, World“ ausgeben. Für ein erfolgreiches Ausführen des oben gezeigten Codeausschnittes muss im Parameter NAO_IP die IP-Adresse des NAOs eingetragen werden, der remote angesteuert werden soll.

Komplexe Datenstrukturen wie zum Beispiel std: : vector<type> oder AL: : ALValue werden nicht mittels zugeschnittener Klassen, sondern durch den generischen Typ Variant repräsentiert. Übergabewerte der Methoden werden nicht als Enumerations, sondern als String übergeben. Eine der auffälligsten Eigenschaften des Java Environments ist das fehlende Eventhandling. So ist es mit Java nicht möglich, sich für Eventbenachrichtigungen $\mathrm{zu}$ registrieren und sich somit über eingetretene Events informieren zu lassen. Um zu testen, ob ein Event eingetreten ist, muss mit der Methode getData () die entsprechende Stelle im Speicher abgefragt und ausgewertet werden. Des Weiteren ist es nicht möglich, eigene NAOqi-Module in Java zu implementieren. 


\subsection{Zwischenfazit}

\begin{tabular}{|l|c|c|c|}
\hline \multirow{2}{*}{ Kriterien } & \multicolumn{2}{c|}{ Entwicklungsumgebung } \\
\cline { 2 - 4 } & Choregraphe & C++ SDK & Java SDK \\
\hline lokal ausführbar & - & ++ & - \\
\hline remote ausführbar & ++ & ++ & ++ \\
\hline Bewegungen entwickeln & ++ & + & + \\
\hline Ausführungsgeschwindigkeit & + & ++ & + \\
\hline $\begin{array}{l}\text { optimiert für eingebettete } \\
\text { Systeme }\end{array}$ & + & ++ & - \\
\hline Eventhandling & + & ++ & - \\
\hline $\begin{array}{l}\text { Betriebssystem } \\
\text { Linux/MAC) }\end{array}$ & ++ & ++ & + \\
\hline Betriebssystem (Windows) & ++ & + & ++ \\
\hline \multicolumn{1}{|c|}{ Ergebnis: } & 11 & 14 & 7 \\
\hline Legende: & ++ trifft zu, + trifft teilweise zu, - trifft nicht zu \\
\hline
\end{tabular}

Tabelle 5 - Bewertung der Softwareeigenschaften

In Tabelle 5 werden die Eigenschaften der drei Entwicklungsumgebungen zusammengefasst und verglichen. Das Ergebnis zeigt die eindeutige Tendenz in Richtung des C++ SDK. Obwohl eine Fernsteuerung des Roboters mit Java per remote möglich ist, ist der Umstand, dass in dieser Programmiersprache keine lokalen Module implementiert und keine Eventbenachrichtigungen durch die NAOqi ausgelöst werden können, ein Ausschlusskriterium. Choregraphe bietet für den Einstieg in die Benutzung ein optimales Werkzeug. Es ermöglicht eine zügige Einarbeitung und schnelle Erfolge bei der Umsetzung. Um eigene Boxen erstellen und komplexe Programmabläufe implementieren zu können, sind Kenntnisse in der Programmiersprache Python von Vorteil. Nichtdestotrotz können auch mit dieser Entwicklungsumgebung keine lokalen Module erstellt werden.

Zusammenfassend ist zu sagen, dass die Entwicklung mit der Programmiersprache $\mathrm{C}++$ die größten Vorteile bietet. Zusätzlich zur Hardwarenähe können mit C++ das Eventhandling und die optimale Ausführungsgeschwindigkeit, welche bei einer Interaktion mit einem Menschen ein wichtiges Kriterium ist, als herausragende Pluspunkte gezählt werden. Der grafische Animationsmodus der Choregraphe Suite ist für die Entwicklung von Bewegungsabläufen das optimale Werkzeug. Die integrierte Funktion der Suite, die erstellten Bewegungen in C++ Quellcode zu exportieren, wird der Autor nutzen, um Emotionen und Gesten mit dem NAORoboter in der Programmiersprache $\mathrm{C}++$ umzusetzen. 


\section{Konzeptentwicklung}

Im folgenden Kapitel wird das Konzept des Autors zur Förderung autistischer Kinder mittels des NAO-Roboters beschrieben. Das Kapitel ist in zwei Unterkapitel gegliedert. Kapitel 5.1 beschreibt allgemeine thematische und technische Voraussetzungen und Anwendungsbedingungen, die inhaltlich für alle drei Teilkonzepte in Kapitel 5.1.1-3 relevant sind. Jedes Teilkonzeptkapitel repräsentiert ein Modul und ist jeweils in einen thematischen und einen technischen Abschnitt unterteilt. Die Abschnitte sind mit separaten Überschriften gekennzeichnet. Basierend auf den vorangegangen Kapiteln 2ff und $3 \mathrm{ff}$ wird im jeweiligen thematischen Teil der Konzeptansatz aus therapeutischer Sicht entwickelt und dargelegt. Im dazugehörigen technischen Abschnitt wird, basierend auf den Grundlagen aus Kapitel 4, unter Einbeziehung der Hardwarekomponenten des NAO und vorhandener NAOqi-Module, die ingenieurtechnische Konzeptentwicklung beleuchtet. Kapitel 5.2 diskutiert, losgelöst von der übrigen Konzeptentwicklung, ob eine Integration des Leap Motion Controllers sinnvoll erscheint.

\section{1. Übersicht}

\section{Thematischer Ansatz}

„Jede Behandlung muß vom individuellen Entwicklungsprofil ausgehen und gezielt von Patient zu Patient jeweils verschieden einzelne Bereiche mit in die Behandlung einbeziehen " [12]

Das heißt, dass eine Anwendungslösung für jedes autistische Kind speziell angepasst werden muss. Wie Helmut Remschmidt ${ }^{22}$ im obigen Zitat belegt, ist bei der Zusammenstellung von Anforderungen für die Konzeption einer Anwendung zur Förderung sozialer Fähigkeiten die jeweilige individuelle Entwicklung des autistischen Kindes zu berücksichtigen.

Das Konzept, welches in dieser Arbeit entwickelt wird, hat den Anspruch, gezielt therapeutisch auf eine einzelne autistischer Kinder anwendbar und doch ganzheitlich zu sein. Das heißt, das Anforderungskonzept für die Anwendung, die auf dem Roboter zum Einsatz kommen soll, ist allgemeingültig als therapeutischer Ansatz zu sehen. Dieser Ansatz soll als Grundlage für weitere Anwendungen auf dem NAO dienen.

Das durch den NAO-Roboter erzeugte, nachgewiesen höhere Konzentrationslevel bei Autisten [27] wird als positive Verstärkung der therapeutischen Maßnahme genutzt. Des

\footnotetext{
${ }^{22}$ Autor des Buches: „Autismus - Erscheinungsformen, Ursachen, Hilfen“, siehe Quelle [29]
} 
Weiteren fließen die drei Hauptbeeinträchtigungen (Kapitel 2.3, Abbildung 2) aus dem autistischen Spektrum mit in die Ausarbeitung des Konzeptes.

\section{Voraussetzungen}

Die Anwendung wird gezielt für Kinder aus dem autistischen Spektrum entwickelt. Dabei sind einige Voraussetzungen nötig, damit eine ergebnisorientierte Förderung sinnvoll im Rahmen der therapeutischen Behandlung ermöglicht werden kann. In der folgenden Übersicht werden die therapeutischen Voraussetzungen an jedes Kind, welches mit dem NAO-Roboter agieren soll, dargestellt und indiziert.

\begin{tabular}{|l|l|}
\hline \multicolumn{1}{|c|}{ Nummer } & \multicolumn{1}{c|}{ Voraussetzung } \\
\hline /V01/ & Autismus ist nach ICD 10 - F84.1 diagnostiziert \\
\hline /V02/ & keine auditiven oder visuellen Defizite \\
\hline /V03/ & keine abnormalen Augenbewegungen \\
\hline /V05/ & ist in der Lage deutsch zu verstehen \\
\hline /V06/ & ist in Lage einfachen Aufforderungen nachzukommen \\
\hline /V07/ & ist im Alter zwischen 6 - 10 Jahren \\
\hline
\end{tabular}

Tabelle 6 - Voraussetzungen für den Erfolg der Anwendung

Nummer /V02/ in Tabelle 6 setzt voraus, dass das Kind keine anatomischen Beeinträchtigungen beim Sehen oder Hören aufweist.

Für den Autor erscheint es sinnvoll für jede der drei Beeinträchtigungen im autistischen Spektrum ein Modul für den NAO-Roboter zu entwickeln. Je nach Fähigkeiten des autistischen Kindes kann vom Therapeut oder Betreuer eines oder mehrere dieser Module zur Verwendung gewählt werden. 


\section{Übersicht}

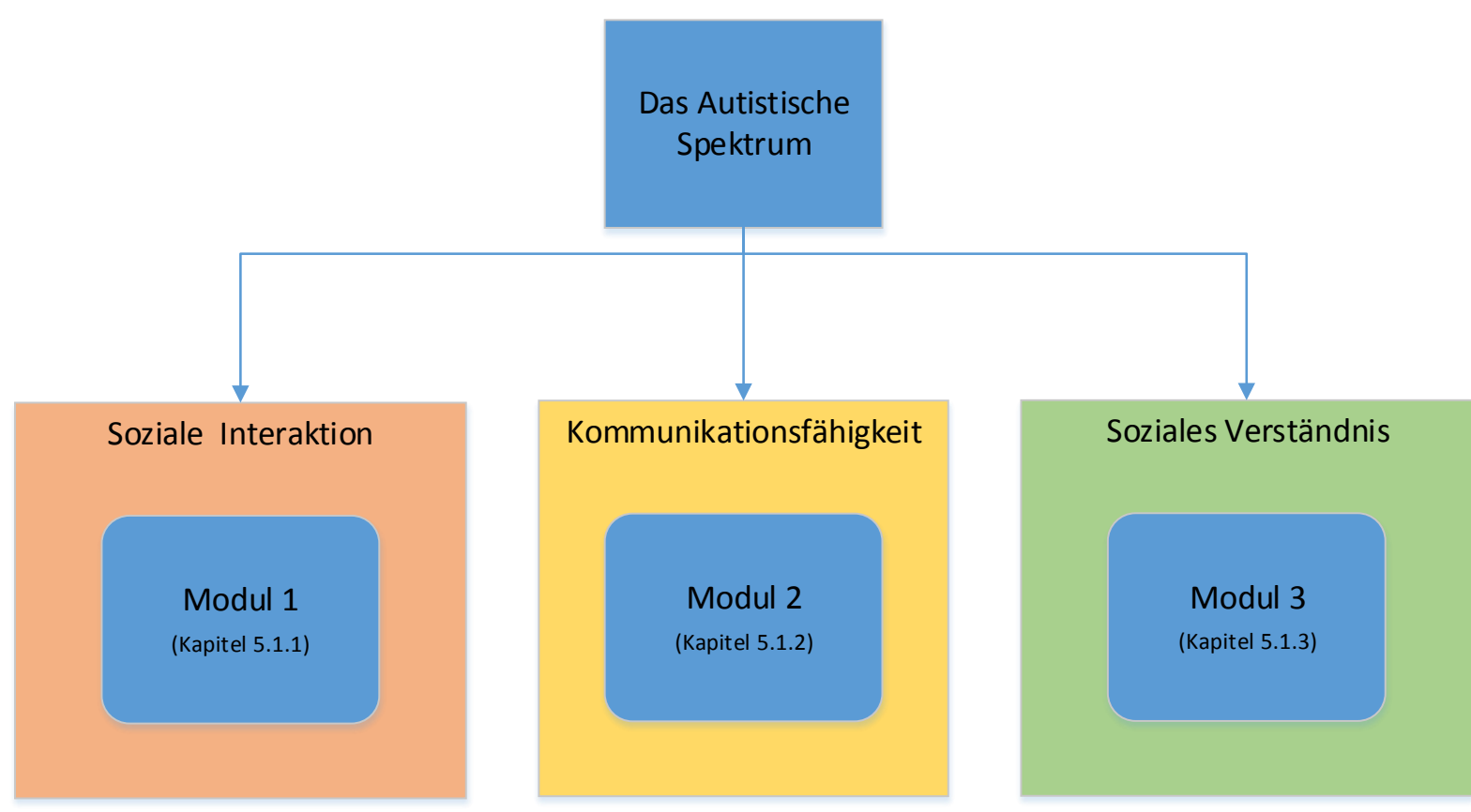

Abbildung 40 - Zuordnung der Module zu Hauptbeeinträchtigungen im autistischen Spektrum

In Abbildung 40 werden vorerst die Module mit 1,2 und 3 durchnummeriert. In den dazugehörigen Kapitelüberschriften wird später der Modulname genannt.

- Modul 1 greift den Schwerpunkt der sozialen Interaktion auf und geht auf eine Förderung der kognitiven Empathie mit Hilfe von Gefühlsimitationen ein.

- Modul 2 ist gesondert zu betrachten, da es in der „Praktischen Studie“ zusammen mit der Bezugsbetreuerin Esther Grohmann in der Ganztagskleinklasse des Therapiezentrums der Schule am Friedrichshain für ein spezielles autistisches Kind angepasst und weiterentwickelt wurde. Anhand dieses Moduls wird der Aufwand für die individuelle Anpassung und Entwicklung einer Anwendung für jedes einzelne Kind aufgezeigt.

- Modul 3 greift den Grundgedanken der Theory of Mind aus Kapitel 2.4 auf und hat die Förderung des sozialen Verständnisses zum Ziel.

Nachfolgend sind Bedingungen und Konzeptbestandteile beschrieben, die für alle drei Module gelten. Sie sind als allgemeine Bedingungen für die Umsetzung im Ganzen zu sehen. 


\section{Raumsetup}

Der Roboter, das Kind und der Therapeut befinden sich zusammen in einem Raum. Der Raum sollte keine ablenkenden Bilder oder auffällige Wandbemalung aufweisen. Der Roboter sitzt oder steht auf einem Tisch an der Wand. Das Kind sitzt vor dem Roboter auf einem Stuhl. Dabei ist ein Sicherheitsabstand von mindestens $60 \mathrm{~cm}$ nötig, um den von Aldebaran Robotics vorgegebenen Aktionsradius (Abbildung 41) des Roboters nicht einzuschränken. Der Betreuer sitzt/steht in unmittelbarer Nähe neben bzw. hinter dem Kind (Abbildung 42).

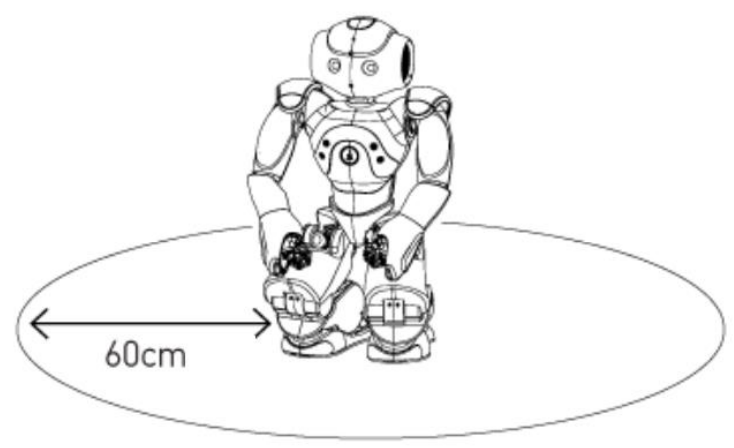

Abbildung 41 - Interaktionsradius des NAO Quelle: Aldebaran Robotics

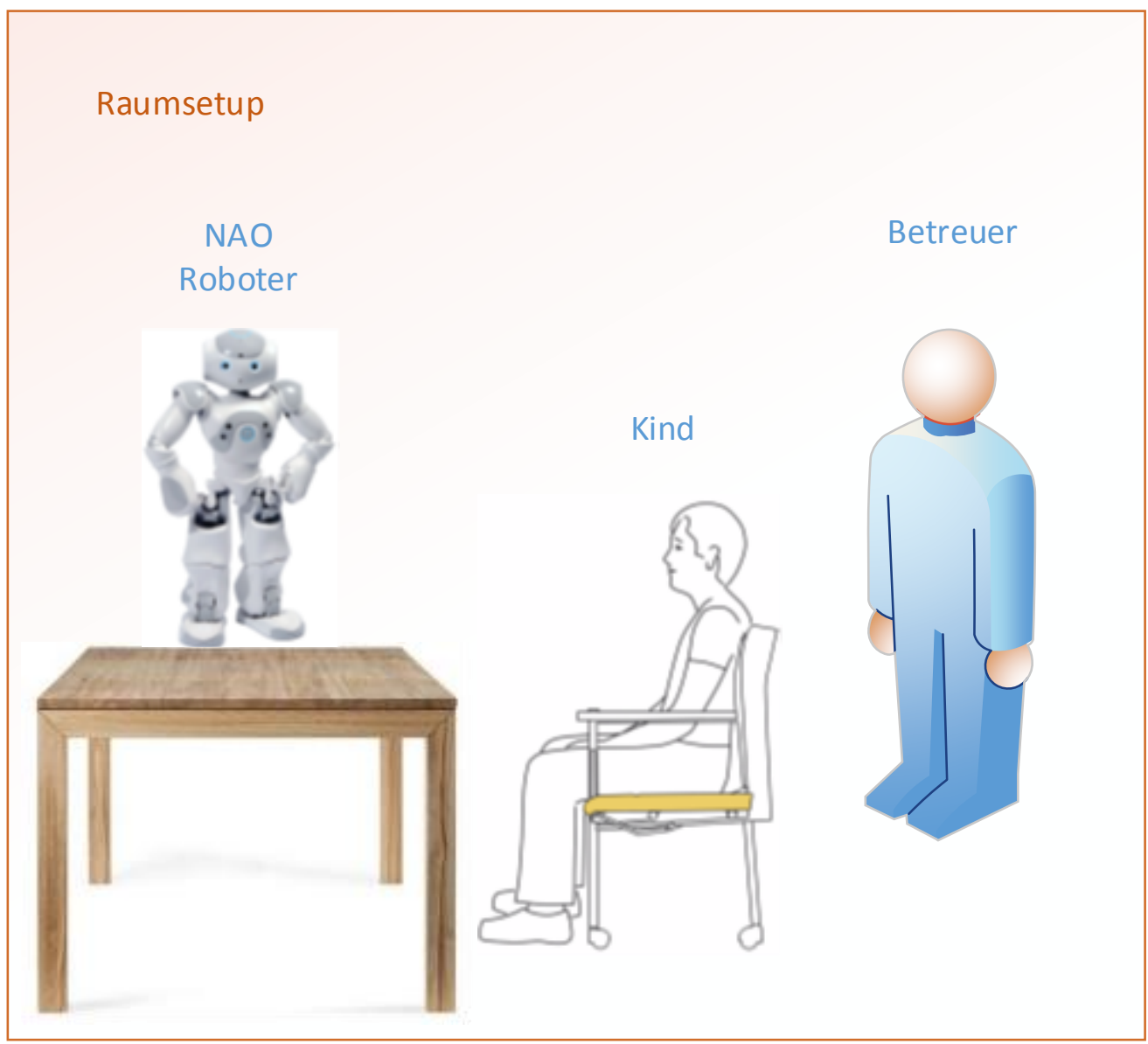

Abbildung 42 - Raumsetup für Sitzungen 


\section{Betreuer/Therapeut}

Der Betreuer wird vor der Sitzung in die Modullogik eingeweiht und wird in die Planung des Ablaufs mit eingebunden. In den ersten Sitzungen ist es sinnvoll, dass sich ein Techniker in der Nähe aufhält, um bei Aussetzern des NAO-Roboters einzugreifen.

\section{Der Roboter}

Um dem Roboter eine Persönlichkeit zu geben, trägt er den Namen „Lino“. Der Name soll für das Kind einfach zu merken sein. Es ist außerdem darauf zu achten, dass Lino vor Beginn einer Therapiesitzung vollständig aufgeladen ist, damit eine kabellose Verwendung möglich ist.

\section{Kommunikation}

Die Sprache des NAO-Roboters muss sehr einfach und klar gehalten werden, damit sie von dem autistischen Kind wahrgenommen wird. Der Roboter sollte langsam sprechen und Wörter benutzen, die mittels des ALTextToSpeech-Moduls von der zuhörenden Person gut verstanden werden können. Der Betreuer darf unterstützend eingreifen, wenn das Kind etwas nicht verstanden hat oder der NAO auf eine Antwort wartet.

\section{Blickkontakt}

Während der Sitzung versucht der NAO-Roboter den Augenkontakt mit dem Kind herzustellen und zu halten. Der NAO soll in der Lage sein, sich das Gesicht des Kindes zu merken und wiederzuerkennen.

\section{Auswertung}

Während der Sitzung zeichnet der NAO-Roboter auf, was seine Kopfkameras erfassen. Diese Aufnahme kann zur späteren Auswertung herangezogen werden, z.B. kann das repetitive Verhalten oder die Dauer des Blickkontakts erfasst und bewertet werden. [12]

\section{Erstkontakt}

Jedes autistische Kind bringt verschieden stark ausgeprägte Verhaltensweisen und Entwicklungsstufen mit in die Therapie. Angepasst an die jeweiligen individuellen Ausprägungen sind entsprechende Förderschwerpunkte zu setzen. Damit eine Therapie mit dem NAO-Roboter Erfolge hervorbringen kann, sollte im Vorhinein eruiert werden, ob und wenn ja bei welchen Interaktionen des Roboters das Kind eine Reaktion zeigt. Damit kann 
herausgefunden werden, an welchen Therapieschwerpunkten angesetzt bzw. welches der drei entwickelten Module als Einstieg in die Therapie verwendet werden sollte.

Für die erste Begegnung zwischen Kind und Roboter werden mit Choregraphe einige Behaviors erstellt, die einen Querschnitt durch das Funktionsspektrum des NAO-Roboters bieten und dabei therapeutische Ansätze der sozialen Kommunikation und Interaktion anreißen. Die Anwendung der Behaviors wird im Kapitel 6.1 - Praktische Studie - näher erläutert. Um beim Erstkontakt zwischen dem Roboter und einem autistischen Kind das Resonanzverhalten des Kindes bei verschiedenen Aktionen und Bewegungen des Roboters auszuwerten, wurde vom Autor eine Wertungsmatrix entwickelt. Mit dieser Wertungsmatrix ist es möglich die Stärke der Reaktionen zu ermitteln, in welchem Bereich der NAO eine besondere Faszination auf das Kind ausübt.

Tabelle 7 zeigt ein Template mit Wertungskriterien und den dazugehörigen Funktionen des Roboters. Das Bewertungstemplate ist für den Erstkontakt zwischen einem autistischem Kind und dem NAO zu verwenden. In Kapitel 6.1 wird die Anwendung des Bewertungsbogens demonstriert. 


\section{Bewertungsbogen für $<$ Name des Kindes $>(<$ Alter $>$ )}

Erklärung der Bewertung

\begin{tabular}{|l|l|}
\hline keine Reaktion & Das Kind steht herum und zeigt keine Reaktion. \\
\hline neutral & Das Kind reagiert erst nach mehrmaligem Auffordern des Therapeuten. \\
\hline wenig interessiert & Das Kind benötigt maximal 2-3 Aufforderungen, um mit dem Roboter zu sprechen/agieren. \\
\hline interessiert & Das Kind spricht/agiert sofort nach Aufforderung des Therapeuten mit dem Roboter. \\
\hline sehr interessiert & Das reagiert sofort auf den Roboter ohne Aufforderung. \\
\hline
\end{tabular}

\begin{tabular}{|c|c|c|c|c|c|c|}
\hline $\begin{array}{c}\text { Wertung } \\
\text { Funktion des NAO }\end{array}$ & $\begin{array}{l}\text { keine } \\
\text { Reaktion }\end{array}$ & neutral & $\begin{array}{l}\text { wenig } \\
\text { interessiert }\end{array}$ & interessiert & $\begin{array}{l}\text { sehr } \\
\text { interessiert }\end{array}$ & Wiederholungen \\
\hline Präsens im Raum (NAO sitzt) & & & & & & \\
\hline Augen blinken / leuchten auf & & & & & & \\
\hline Bewegt Kopf nach rechts und links & & & & & & \\
\hline spricht deutsch: "Ist dort jemand?" & & & & & & \\
\hline $\begin{array}{l}\text { spricht englisch: "Is anybody } \\
\text { there?" }\end{array}$ & & & & & & \\
\hline steht aus der Sitzposition auf & & & & & & \\
\hline spielt eine Musikdatei ab & & & & & & \\
\hline winkt im Stehen & & & & & & \\
\hline $\begin{array}{l}\text { NAO fordert auf den Fuß zu } \\
\text { berühren }\end{array}$ & & & & & & \\
\hline
\end{tabular}

Für die Auswertung reicht es, ein $\mathrm{X}$ in das jeweilige Feld zu setzen.

Die Anzahl der Wiederholungen wird als Zahl eingetragen.
Datum

Eine erste Interaktion mit dem Roboter NAO

C Benjamin Körner 
Nachfolgend wird der allgemeine technische Ansatz beschrieben.

\section{Technischer Ansatz}

\section{Auswahl der Entwicklungsumgebung}

Ausgehend vom Zwischenfazit der Softwareevaluierung in Kapitel 4.3 nutzt der Autor die Programmiersprache $\mathrm{C}++$, um die drei Module als lokale Module zu implementieren. So lassen sich, wie in Kapitel 4.2 .2 beschrieben, die größten Vorteile der hardwarenahen Programmierung umsetzen. Dafür wird die, ebenfalls in Kapitel 4.2.2 beschriebene, virtuelle Maschine von Georg Labbé [47] verwendet. Für die Erstellung von motorischen Bewegungsmustern wird der Animationsmodus der Software Choregraphe verwendet werden.

\section{Ausgangssituation}

Für die technische Konzeptentwicklung ist es wichtig zu erwähnen, dass 2014 im Studiengang Telematik der TH Wildau im 6.Semester im Fachmodul „Embedded C++“ drei Studenten (der Autor eingeschlossen) ein Konzept entwickelt haben, um neue, für den NAO entwickelte Module durch ein Startmodul automatisch erkennen und ausführen zulassen. Dabei wird das sogenannte „Moderator“-Modul über den mittleren Kopfsensor des Roboters gestartet und erkennt dynamisch alle Module, die den weiter unten beschriebenen Konventionen zur Entwicklung neuer Module entsprechen. Das Starten der einzelnen Module erfolgt über Sprachanweisungen der Person, die mit dem Roboter interagiert. Dabei muss dem Roboter der gewünschte Modulname gesagt werden. Für die Erkennung der Sprachanweisung ist die NAOqi-eigene ALSpeechRecognition-Bibliothek zuständig. Die Konventionen für selbst entwickelte Module, die vom „Moderator“-Modul dynamisch erkannt werden sollen, lauten wie folgt:

\section{Konventionen:}

- Der Modulname muss das Präfix „, THWild_Game_"beinhalten

- Das Modul muss eine Methode getGameName() besitzen. Diese Methode muss an das Modul gebunden sein, damit sie öffentlich zugänglich ist. Die Methode soll den Spielnamen als Rückgabewert liefern.

- Das Modul muss eine Methode startGame() besitzen. Diese Methode muss an das Modul gebunden sein, damit sie öffentlich zugänglich ist. Diese Methode dient als Einstiegspunkt für das Spiel. 
Dieser Ansatz bietet eine flexible Methodik, um effizient und unkompliziert neue Module zum Repertoire hinzuzufügen. Auf die visuelle Darstellung der Programmlogik des „Moderator“-Moduls, wurde der Übersicht halber an dieser Stelle verzichtet. Der Programmablaufplan kann im Anhang in Anlage A.2. eingesehen werden. Wichtig zu erwähnen ist, dass das „Moderator“-Modul die Gesichtserkennung, das Facetracking und die Videoaufnahmefunktion bereitstellt. Die Funktionen werden nachfolgend beschrieben.

\section{Allgemeine Hardwareanforderungen}

Tabelle 8 werden die Hardwareanforderungen an den Roboter aufgelistet, die in allen drei Modulen und dem „Moderator“-Modul Anwendung finden. In den Unterkapiteln 5.1.1-3 wird jeweils auf einzelne spezielle NAOqi-Bibliotheken separat eingegangen.

\begin{tabular}{|c|c|c|c|}
\hline Nr. & Aktion des NAO & Fähigkeit & Hardware \\
\hline /HA01/ & $\begin{array}{l}\text { Blickkontakt } \\
\text { herstellen }\end{array}$ & Gesichtserkennung & Kameras \\
\hline /HA02/ & $\begin{array}{l}\text { Blickkontakt } \\
\text { halten }\end{array}$ & Facetracking & Kameras \\
\hline /HA03/ & $\begin{array}{l}\text { Aufforderungen } \\
\text { erteilen, Regeln } \\
\text { erklären, } \\
\text { Begrüßung }\end{array}$ & Sprachausgabe & Lautsprecher \\
\hline /НА04/ & $\begin{array}{l}\text { Modulnamen und } \\
\text { Antworten des } \\
\text { Kindes verstehen }\end{array}$ & Spracherkennung & Mikrofone \\
\hline /HA05/ & Auswertung & Aufzeichnen der Sitzung & Kameras \\
\hline
\end{tabular}

Tabelle 8 - Hardwareanforderungen, die für alle Module gelten

Für die Gesichtserkennung (HA01) werden die beiden Kameras an der Frontseite des Kopfes des NAO-Roboters verwendet. Das Facetracking wird über die obere der beiden Kameras realisiert. Für die Sprachausgabe (HA02) dienen die Lautsprecher an den Kopfseiten des NAO-Roboters. Die Spracherkennung (HA03) erfolgt über die Mikrofone, wie sie in Abbildung 22 in Kapitel 4.1.3 zu sehen sind. Für die Auswertung und Aufzeichnung (HA04) von Videodateien werden ebenfalls die Kameras verwendet.

\section{Notwendige NAOqi-Module}

Um die vorangegangen konzeptionierten Module implementieren zu können, bedarf es der Verwendung sämtlicher in Tabelle 2 in Kapitel 4.2 aufgeführten NAOqi-Bibliotheken. Diese sind in Tabelle 10 auf der nächsten Seite nach gleichem Muster wie Tabelle 2 und unter den gleichen Kategorien zusammengefasst. Um die Struktur der Arbeit übersichtlich zu halten, 
werden je nach Schwerpunktsetzung der drei Module „Gefühle“, Berühre Mich“ und „Geschichte“ die einzelnen NAOqi-Bibliotheken durch einen Farbcode zugeordnet. Dazu werden in jedem Unterkapitel die jeweils zu verwendenden NAOqi-Bibliotheken beschrieben. Folgende Farbzuordnung basiert auf der Konzeptübersicht aus Abbildung 40.

\begin{tabular}{|l|}
\hline Farbcode \\
\hline Modulübergreifend \\
\hline Modul 1 „Gefühle“ \\
\hline Modul 2 „Berühre Mich“ \\
\hline Modul 3 „Geschichte“ \\
\hline
\end{tabular}

Tabelle 9 - Farbcodezuordnung für die einzelnen Module

Die hellblaue Markierung fasst alle Bibliotheken und Events zusammen, die modulübergreifend eingesetzt werden. Sie werden in den einzelnen Modulkapiteln nicht nochmals erläutert. Die anderen drei Farben sind selbsterklärend.

\begin{tabular}{|l|}
\hline Core \\
\hline ALMemory \\
\hline ALModule \\
\hline
\end{tabular}

\begin{tabular}{|l|}
\hline Audio \\
\hline ALSpeechRecognition \\
\hline ALTextToSpeech \\
\hline ALAudioplayer \\
\hline
\end{tabular}

\begin{tabular}{|l|}
\hline Sensors \\
\hline ALLeds \\
\hline ALSensors \\
\hline
\end{tabular}

\begin{tabular}{|l|}
\hline Tracker \\
\hline ALFaceTracker \\
\hline
\end{tabular}

\begin{tabular}{|l|}
\hline Vision \\
\hline ALFacedetection \\
\hline ALMovementDetection \\
\hline ALVideoRecorder \\
\hline
\end{tabular}

\section{Motion}

ALMotion

Tabelle 10 - Notwendige NAOqi-Module

Äquivalent dazu wird mit den von der NAOqi-API bereitgestellten Eventbenachrichtigungen verfahren. Entsprechend Tabelle 4 in Kapitel 4.2.2 wird in nachfolgender Tabelle 11 die farbliche Zuordnung zu den Modulen deutlich.

\begin{tabular}{|l|}
\hline Audio \\
\hline WordRecognized \\
\hline
\end{tabular}

\begin{tabular}{|l|}
\hline Sensors \\
\hline FrontTactilTouched \\
\hline HandLeftBackTouched \\
\hline HandLeftLeftTouched \\
\hline HandLeftRightTouched \\
\hline HandRightBackTouched \\
\hline HandRightLeftTouched \\
\hline HandRightRightTouched \\
\hline LeftBumperPressed \\
\hline MiddleTactilTouched \\
\hline RearTactilTouched \\
\hline RightBumperPressed \\
\hline
\end{tabular}

\begin{tabular}{|l|}
\hline Vision \\
\hline FaceDetected \\
\hline MovementDetected \\
\hline
\end{tabular}


Nachfolgend werden die einzelnen modulübergreifenden NAOqi-Bibliotheken für das spätere Verständnis in der Umsetzung erörtert.

\section{ALMemory}

Die NAOqi-Bibliothek ALMemory steht als zentrale Speicherstelle zur Verfügung. Sie kann benutzt werden, um angelegte Werte abzuspeichern oder auszulesen. Weiterhin fungiert sie als Verteiler und stellt alle Eventbenachrichtigung zur Verfügung. Diese Eigenschaft ist notwendig, um sich über Events wie z.B. der Berührung eines Sensors oder die Erkennung eines Gesichtes informieren zu lassen.

\section{ALModule}

Die ALModule-API dient als Vorlage für alle lokal erstellten Module. Sie stellt eine Basisklasse zur Verfügung, die bei der Bereitstellung und Bekanntmachung von eigenen Methoden behilflich ist. Die Klasse ist nötig, um eigene Module zu erstellen.

\section{ALFaceDetection}

ALFaceDetection erkennt, ein menschliches Gesicht, welches sich vor dem NAO-Roboter befindet. Optional lässt sich das Gesicht speichern und wiedererkennen. Dabei werden die Positionen der Hauptmerkmale wie Augen, Augenbrauen, Nase und Mund in einer Liste gespeichert. Ein Modul registriert sich am Event FaceDetected und lässt sich beim Eintreten desselbigen benachrichtigen.

\section{ALFaceTracker}

Das ALFaceTracker-Modul kann ein erkanntes Gesicht verfolgen. Es schlägt eine Brücke zwischen Gesichtserkennung und Bewegung des Kopfes, um ein erkanntes Objekt möglichst immer in der Mitte des Kamerabildes zu halten. Voraussetzung für das Tracking eines Gesichts ist das Erkennen eines solchen durch das ALFaceDetection-Modul.

\section{ALVideorecorder}

Das ALVideorecorder-Modul stellt Methoden bereit, um über die Kopfkameras des NAOs Filmsequenzen aufzunehmen. Die Videodatei wird während der Aufzeichnung im Linux-Dateisystem auf dem NAO unter /home/nao/recordings/ als AVI*-Datei abgelegt. Zu beachten gilt es, dass immer nur eine Aufzeichnung zur gleichen Zeit möglich ist. 
Nachfolgend werden die Konzepte der Module 1,2 und 3 als Teilkonzepte dargestellt. Jedes dieser Module ist als Vorlage zu betrachten und kann individuell nach Anforderungen variiert werden.

\subsubsection{Teilkonzept 1 - Modul 1 „Gefühle“}

\section{Thematischer Ansatz}

Wie in Kapitel 2 beschrieben, fehlt es Autisten an kognitiver und emotionaler Empathie. Sie weisen große Defizite im Bereich der sozialen Kommunikation und Interaktion auf. Nach neuesten Erkenntnissen ist eine Störung der Spiegelneuronen die neurologische Ursache [48]. Viele Autisten haben dafür oft ein ausgeprägtes mathematisches und analytisches Verständnis. Dieses Teilkonzept der Arbeit untermauert unter anderem die These, dass Emotionen und Empathie analytisch durch Imitation einer anderen Person oder Roboter, erlernt werden können.

Kapitel 3.4 beschreibt drei Szenarien für den Einsatz des Roboters. Das dreiteilige Interaktionsmodell von Mustafa S. Erden wird als Grundlage für dieses Teilkonzept adaptiert, da es einen größeren variablen sozialen Kontext als ein zweiteiliges Modell ermöglicht. Der NAO-Roboter agiert als Mediator zwischen dem Therapeuten und dem autistischen Kind.

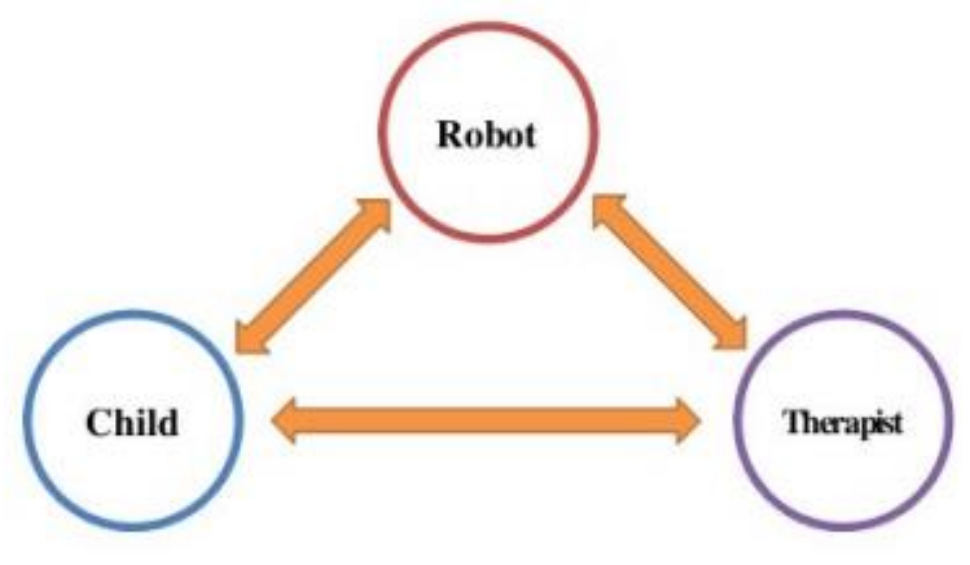

Abbildung 43 - dreiteiliges Interaktionsmodell Quelle: [22]

Das dreiteilige Interaktionsmodell (Abbildung 43) wird in verschiedene Aktoren unterteilt. Hierbei wird den Aktoren (Kind, Therapeut und Roboter) jeweils eine Rolle im Szenario zugeteilt: die des Initiators (startet eine Interaktion oder zeigt eine neue Bewegung), die des Responders (empfängt das soziale Signal und/oder imitiert die Bewegung des Initiators) und die des Zuschauers (beobachtet die Interaktion der beiden anderen Aktoren). Die 
verschiedenen indirekten Rollenzuweisungen im Zuge der Interaktion während der Sitzung bringen den Vorteil, dass das autistische Kind die Situation aus verschiedenen Blickwinkeln erfassen kann (Tabelle 12).

In Studie 3 (siehe Kapitel 3.4) wurden durch Mustafa S. Erden ebenfalls drei Emotionen herausgearbeitet, die mit dem NAO-Roboter am eindeutigsten erkennbar zu realisieren sind. Dafür hat er insgesamt 30 Gesten mit dem Roboter erstellt und die jeweils fünf eindeutigsten für die drei Emotionen „,wütend“, „traurig“ und ,glücklich“ in den Abbildungen 11, 12 und 13 in Kapitel 3.4 dargestellt. Für das Konzept wurden die Figuren aus diesen Abbildungen extrahiert, die dem Autor am ehesten als Vorlage für eine Implementierung dienlich erscheinen (Abbildung 44).
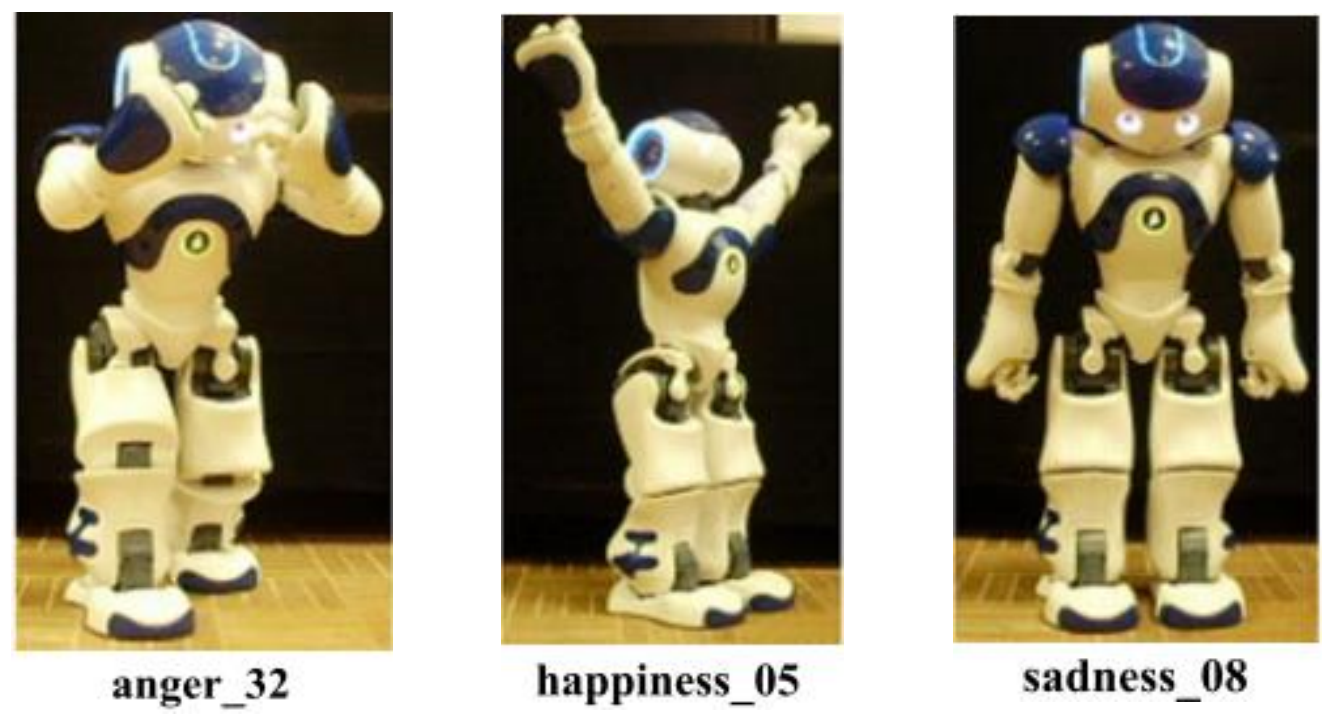

Abbildung 44 - Extraktion der Emotionen "wütend", "glücklich" und "traurig" Quelle: [22]

Der Roboter ist in der Lage, diese drei Basisemotionen als Gesten so darzustellen, dass die entsprechend $\mathrm{zu}$ übertragenen Emotionen eindeutig von Menschen identifiziert werden können [37].

Der Schwerpunkt des Moduls liegt auf der Förderung der kognitiven Empathie. Das autistische Kind soll Schritt für Schritt analytisch durch den Modulaufbau lernen, die Begriffe „wütend“, „traurig“ und „glücklich“ bestimmten Gesten und Bewegungen zuzuordnen und diese zu erkennen. Das Einfühlungsvermögen zu schulen und die sozialen Signale zu deuten sind weitere Förderschwerpunkte des Moduls. Zu jeder Emotion wird der NAO-Roboter das Gefühl zusätzlich mit den Worten „Ich bin so glücklich.“ bzw. „Ich bin traurig.“ oder „Ich bin jetzt wütend.“ verbalisieren. Dabei ist darauf zu achten, dass die Intonation der Stimme an die jeweilige Stimmung angepasst wird. 
In und Abbildung 45 auf den folgenden Seiten werden die Rollenverteilung und die Logik des Konzepts deutlich. Hier sieht man, dass jeder Aktor je nach Situation verschiedene Rollen zugewiesen bekommt. Somit kann innerhalb einer Sitzung der soziale Kontext variieren. Das ist wichtig, damit das Kind z.B. die soziale Interaktion zwischen dem Roboter und dem Therapeuten beobachten kann. Dies könnte die Aufmerksamkeit des Kindes beim Beobachten bestimmter Handlungen anderer Menschen fördern. Gleichzeitig kann es üben zu verstehen, wann ein Sprecher in einem Gespräch an der Reihe ist. Das Kind soll die Aufmerksamkeit auch auf den Therapeuten richten, da es sich in Gesellschaft mit anderen Menschen verständigen soll. Aus Tabelle 12 ist ebenfalls ersichtlich, dass das Modul auch Therapieschwerpunkte aus dem Bereich der Kommunikation und des sozialen Verständnisses abdeckt. Die Grenzen der drei Hauptkategorien verschwimmen und gehen oft nahtlos ineinander über. Dieser Umstand kann für die Förderung der Fähigkeiten autistischer Kinder nur von Vorteil sein, dem er dem ganzheitlichen Förderansatz entspricht. In der letzten Spalte der Tabelle 12 werden die Förderziele beschrieben. 


\begin{tabular}{|c|c|c|c|c|c|}
\hline \multirow{2}{*}{ Phase } & \multicolumn{3}{|c|}{ Rollenverteilung } & \multirow{2}{*}{ Beschreibung } & \multirow{2}{*}{ Ziel } \\
\hline & Kind & Therapeut & Roboter & & \\
\hline Begrüßung & Beobachter & Initiator & Responder & $\begin{array}{l}\text { Der Therapeut sagt "Hallo Lino." und winkt. } \\
\text { Der Roboter winkt zurück und sagt "Hallo, wie } \\
\text { geht es euch." }\end{array}$ & Aufmerksamkeit des Kindes wecken \\
\hline Kommunikation & Responder & Beobachter & Initiator & $\begin{array}{l}\text { Der Roboter fragt, ob die Regeln des Spiels } \\
\text { bekannt sind. Antwortet das Kind mit "nein", } \\
\text { werden die Regeln erklärt. Bei "ja" wird zur } \\
\text { nächsten Phase gesprungen. }\end{array}$ & Zuhören und verstehen \\
\hline $\begin{array}{c}\text { geteilte } \\
\text { Aufmerksamkeit }\end{array}$ & Beobachter & Beobachter & Initiator & $\begin{array}{l}\text { Der Roboter zeigt nacheinander die drei } \\
\text { Emotionen "wütend", "traurig" und "glücklich" } \\
\text { mit Bewegungen und kommentiert diese verbal. }\end{array}$ & $\begin{array}{l}\text { Aufmerksamkeit für Handlungen } \\
\text { anderer erhöhen, Emotionen mit } \\
\text { Gesten verknüpfen, soziale Signale } \\
\text { deuten, Empathie-Empfinden fördern }\end{array}$ \\
\hline $\begin{array}{c}\text { Gestik } \\
\text { und Emotionen }\end{array}$ & $\begin{array}{l}\text { Beobachter } \\
\text { / Responder }\end{array}$ & Beobachter & Initiator & $\begin{array}{l}\text { Der Roboter zeigt eine Bewegung/Gestik und } \\
\text { erwartet vom Kind die Antworten "wütend", } \\
\text { "traurig" oder "glücklich". }\end{array}$ & $\begin{array}{l}\text { Emotionen lernen, Gesten } \\
\text { wiedererkennen }\end{array}$ \\
\hline Imitation & $\begin{array}{l}\text { Beobachter } \\
\text { / Responder }\end{array}$ & $\begin{array}{l}\text { Beobachter } \\
\text { / Responder }\end{array}$ & Initiator & $\begin{array}{l}\text { Der Roboter ermuntert den Therapeut und das } \\
\text { Kind seine Bewegungen/Gesten nachzumachen. }\end{array}$ & $\begin{array}{l}\text { Imitation lernen, Emotionen mit } \\
\text { Bewegungen verknüpfen, Empathie- } \\
\text { Empfinden fördern }\end{array}$ \\
\hline Vertiefung & Responder & Initiator & Beobachter & $\begin{array}{l}\text { Der Therapeut macht dem Kind nun einige } \\
\text { Bewegungen/Gesten vor und bitte es diese } \\
\text { nachzuahmen. Es sollten die drei selben Gesten } \\
\text { sein, die der Roboter vorher initiierte. }\end{array}$ & $\begin{array}{l}\text { Imitation lernen, Emotionen mit } \\
\text { Bewegungen verknüpfen, Gesten } \\
\text { eines Menschen interpretieren, soziale } \\
\text { Signale deuten, Empathie-Empfinden } \\
\text { fördern }\end{array}$ \\
\hline Verabschiedung & $\begin{array}{l}\text { Beobachter } \\
\text { / Responder }\end{array}$ & Initiator & Responder & $\begin{array}{l}\text { Der Therapeut berührt den rechten Fuß des } \\
\text { Roboters und sagt "Auf Wiedersehen, Lino" und } \\
\text { winkt. Der Roboter winkt zurück und sagt "Auf } \\
\text { Wiedersehen, bis zum nächsten Mal". }\end{array}$ & $\begin{array}{l}\text { Aufmerksamkeit für Handlungen } \\
\text { anderer erhöhen }\end{array}$ \\
\hline
\end{tabular}



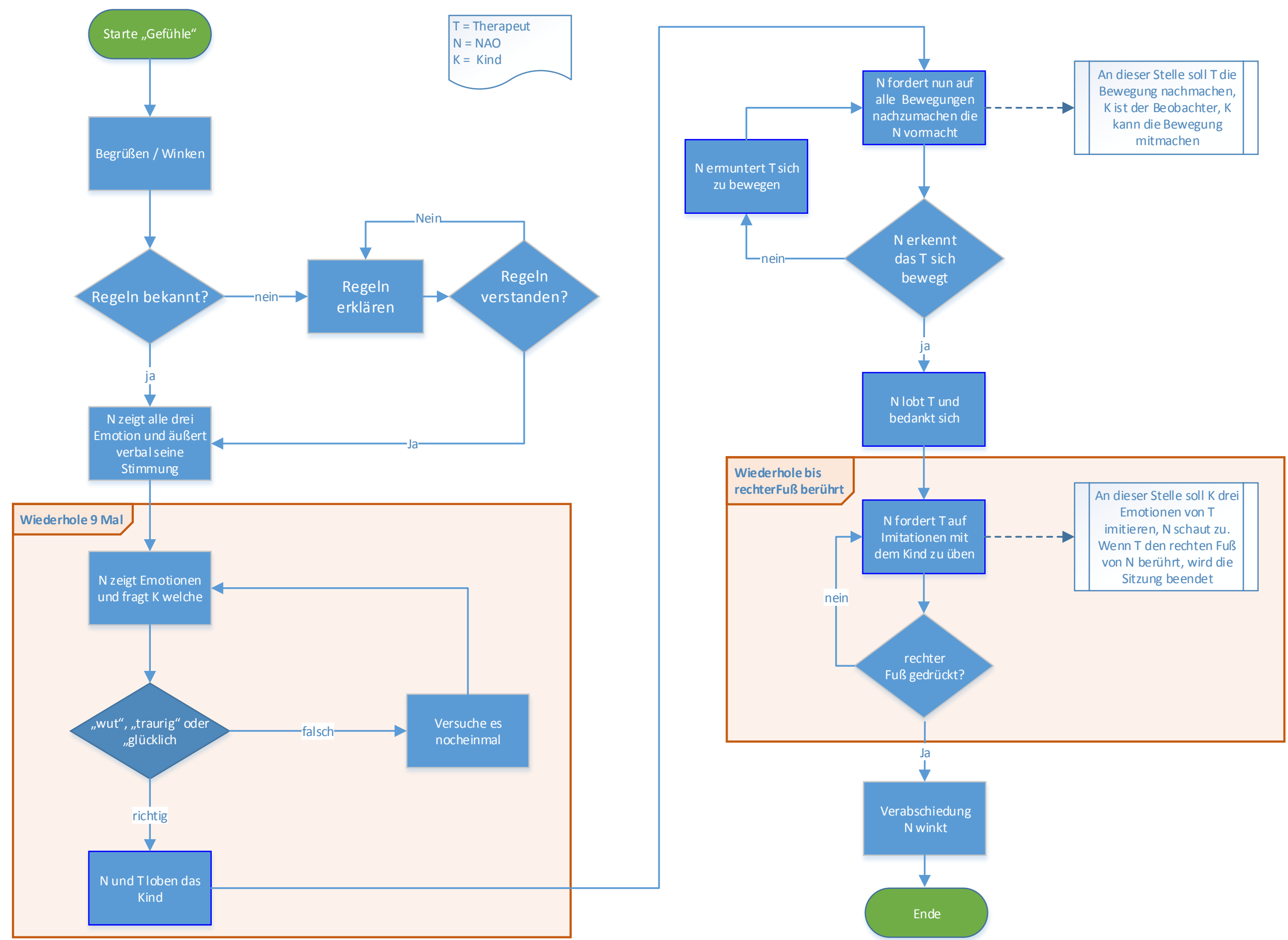

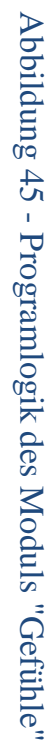




\section{Technischer Ansatz}

In Tabelle 12 sind weitere Hardwarekomponenten aufgelistet, die in Modul 1 vorrangig genutzt werden.

\begin{tabular}{|c|c|c|c|}
\hline Nr. & Aktion des NAO & Fähigkeit & Hardware \\
\hline /M1/HA01/ & Gesten darstellen & Motorik und Bewegung & Motoren und Gelenke \\
\hline /M1/HA02/ & $\begin{array}{l}\text { Zwinkern und } \\
\text { Farbstimmung der } \\
\text { Augen }\end{array}$ & Augenmimik & LEDs \\
\hline /M1/HA03/ & $\begin{array}{l}\text { Antworten des } \\
\text { Kindes erkennen }\end{array}$ & Spracherkennung & Mikrofone \\
\hline /M1/HA04/ & Berührung am Fuß & Sensorauswertung & $\begin{array}{l}\text { Mechanische Sensoren am } \\
\text { Fuß }\end{array}$ \\
\hline
\end{tabular}

Tabelle 13 - Anforderungen an Modul 1

Die Anzahl der in /M1/HA01/ benutzten Motoren und Gelenke hängt von der Komplexität der Umsetzung der Gesten ab. Die LEDs in den Augen des NAOs werden für die Farbstimmung (M1/HA03/) genutzt. Um das Programm zu unterbrechen, wird der rechte Fußsensor (M1/HA04/) verwendet.

Um die Gesten „,wütend“, „traurig“ und „glücklich“ darzustellen, ist eine Vorgehensweise nötig, die den Zeit-Nutzen-Aufwand zur Erstellung dieser Gesten beachtet. Der bereits vorgestellte Animationsmodus bietet sich hier als optimales Tool an. Durch die visuelle Unterstützung bei der Gestenerstellung und die mögliche Exportfunktion in C++ Quellcode wird es möglich, die drei umzusetzenden Gesten in ein lokales, in $\mathrm{C}++$ geschriebenes Modul zu integrieren (Siehe Anlage A.1.) .

Nachfolgend werden die NAOqi-Bibliotheken, die in diesem Modul verwendet werden, erörtert.

\section{ALMovementDetection}

Das Modul ALMovementDetection wird für die Erkennung von Bewegungen in der Nähe des Roboters mittels der Kameras eingesetzt. Dabei wird die CPU laut dem Hersteller stark in Anspruch genommen. Es wird nicht empfohlen, die Bewegungserkennung gleichzeitig mit $\mathrm{zu}$ vielen anderen Modulen $\mathrm{zu}$ benutzen. Außerdem sollte darauf geachtet werden, dass die Eventbenachrichtigung MovementDetected, während der NAO sich selbst bewegt, ignoriert wird. 
ALLeds

Das ALLeds-Modul erlaubt die Kontrolle über die LEDs an den Ohren, auf dem Kopf, um die Augen und an den Füßen. Verwendet werden in diesem Modul die LEDs in den Augen des NAO-Roboters Diese können jede RGB*-Farbkombination annehmen. Die Intensität kann beliebig zwischen 0 und $100 \%$ gewählt und jedes Auge einzeln angesprochen werden.

\section{ALMotion}

Die NAOqi-Bibliothek ALMotion stellt Methoden bereit, welche komplexe Bewegungen des NAO ermöglichen. Bei jeder Anfrage an die ALMotion-API wird eine sogenannter motion task erstellt, der den Bewegungsablauf überwacht und ausführt. Wichtig für die Umsetzung der Gesten in diesem Modul sind die Methoden angleInterpolationBezier (names, times, keys) und angleInterpolation (names, times, keys). Mit diesen Methoden können die durch den Animationsmodus erstellten und exportierten Motions, im C++ Quellcode ausgeführt werden. 


\subsubsection{Teilkonzept 2 - Modul 2 „Berühre mich“}

\section{Thematischer Ansatz}

Geteilte Aufmerksamkeit ist ein Schlüsselaspekt für die soziale Kommunikation. In einer Studie von E. Bekele an der Vanderbilt Universität in Nashville [41] wird davon gesprochen, dass die Förderung der sozialen Kommunikation durch ein Erreichen der geteilten Aufmerksamkeit in spielerischen Anwendungen die besten Ergebnisse verspricht.

Die Berührung eines anderen Menschen ist ein weiterer Schlüsselaspekt der nonverbalen Kommunikation. Mit einfachsten Berührungen können Autisten wie auch andere Menschen Bedürfnisse zeigen und Initiator einer sozialen Kommunikation sein. Das in diesem Teilkonzept vorgestellte Modul ist vom Autor dieser Arbeit entwickelt worden, um eine berührungsorientierte Interaktion mit dem NAO zu realisieren und die Berührungsängste dem Roboter gegenüber zu mindern. Das Teilkonzept wird vom NAO als Spiel initiiert, da eine spielerische Situation für ein Kind mehr Vertrautes birgt und es dadurch Ungewohntes schneller annimmt.

Im Modul „Berühre Mich“ fordert der NAO im Sitzen seinen Gegenüber verbal auf ihn an bestimmten Körperteilen zu berühren. Folgende Berührungspunkte werden per Zufall ausgewählt:

- Linker und rechter Fuß

- Stirn und Hinterkopf

- Linke Hand an den Seiten und auf dem Handrücken

- Rechte Hand an den Seiten und auf dem Handrücken

Während des Spiels versucht der NAO Blickkontakt mit dem Kind herzustellen und zu halten. Die dafür notwendige Technik wird im technischen Konzeptteil erklärt. Die richtige Berührung der Körperstellen wird durch ein Feedback honoriert, welches abhängig vom individuellen Profil des autistischen Kindes angepasst werden kann. Das in diesem Kapitel vorgestellte Modul reagiert mit einem Sound (ein kurzes Klatschen) und einem verbalen Lob des Roboters auf die richtige Berührung. Nach zehn Durchläufen endet das Spiel und das Kind wird gefragt, ob es noch einmal spielen möchte. Die Modullogik ist in Abbildung 46 visualisiert. 

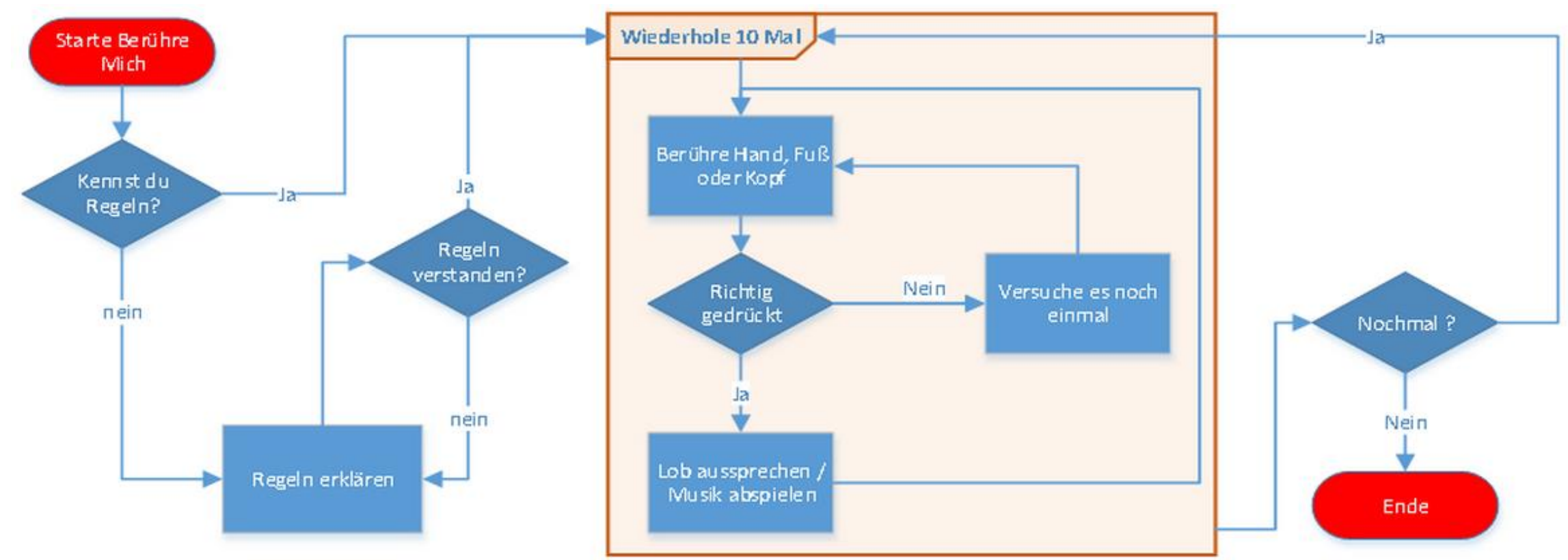

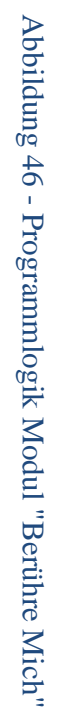


Die Förderziele dieses Moduls sind unter anderem:

- Berührungsängste zu überwinden

- verbale soziale Signale zu verstehen

- Aufforderungen nachzukommen

- stereotypes Verhalten zu mindern

- geteilte Aufmerksamkeit zu fördern

- und Blickkontakt zu halten

Im Kapitel 6.1 „Praktische Studie“ wird anhand des Entwicklungsprozesses während der Testphasen beispielhaft aufgezeigt, wie einzelne Entwicklungsschritte zum Umsetzen einer individuellen Anwendung für ein ausgewähltes Kind aussehen können. Dafür wird die in Abbildung 47 eben gezeigte Programmlogik verwendet und individuell angepasst.

\section{Technischer Ansatz}

Tabelle 13 listet die primären Hardwareanforderungen an das Modul 2 auf.

\begin{tabular}{|l|l|l|l|}
\hline Nr. & Aktion des NAO & Fähigkeit & Hardware \\
\hline /M2/HA01/ & $\begin{array}{l}\text { Körperteil } \\
\text { bewegen }\end{array}$ & Motorik und Bewegung & Motoren und Gelenke \\
\hline /M2/HA02/ & $\begin{array}{l}\text { Abspielen von } \\
\text { Sounddateien }\end{array}$ & Soundwiedergabe & Lautsprecher \\
\hline /M2/HA03/ & $\begin{array}{l}\text { Berührung der } \\
\text { Körperstellen }\end{array}$ & Sensorauswertung & $\begin{array}{l}\text { mechanische und taktile } \\
\text { Sensoren }\end{array}$ \\
\hline
\end{tabular}

Tabelle 14 - Anforderungen an Modul 2

Der Fokus dieses Moduls liegt auf der korrekten Sensorauswertung der zu berührenden Sensoren (/M2/HA03). Die Wiedergabe von Audiodateien (/M2/HA02/) erfolgt über die Lautsprecher des NAO. Die Bewegung von Körperteilen (/M2/HA01/) wird in dieser Tabelle der Vollständigkeit halber mit aufgeführt. Die Implementierung, welche Bewegungen wann ausgeführt werden, wird am Ende der praktischen Studie erläutert.

Nachfolgend werden die NAOqi-Bibliotheken und die zugehörigen Eventbenachrichtigungen, die in diesem Modul verwendet werden, erörtert.

\section{ALAudioPlayer}

Die ALAudioPlayer API bietet Methoden, die das Abspielen, Stoppen und Pausieren von verschiedenen Audioformaten, wie z.B. WAVE* oder MP3*, 
ermöglichen. Dieses Modul ist nur auf dem NAO-Roboter lokal ausführbar. Zu beachten ist, dass eine unkomprimierte WAVE Datei mehr Zeit benötigt, um von der API geladen und abgespielt zu werden, als eine MP3-Datei.

\section{ALSensor}

Die ALSensor-Bibliothek ist für das Eventmanagement der taktilen und mechanischen Sensoren verantwortlich. Sie liest die Eventdaten aus dem Speicher ALMemory aus und löst, je nach geforderter Situation, einzelne Events aus. Die Auflistung auf der nächsten Seite ist auf die in diesem Modul 2 nötigen und auszuwertenden Events gekürzt. Um eine vollständige Eventauflistung der ALSensor API zu erhalten, wird auf den Link in der Fußnote verwiesen. ${ }^{23}$

Fußspitzen:

- RightBumperPressed

- LeftBumperPressed

Kopfsensoren:

- FrontTactiltouched

- RearTactilTouched

Rechte Hand:

- HandRightBackTouched

- HandRightLeftTouched

- HandRightRightTouched

Linke Hand:

- HandLeftBackTouched

- HandLeftLeftTouched

- HandLeftRightTouched

Obige Events werden im Modul 2 ausgewertet. Durch das Auslösen der jeweiligen gleichnamigen Callback-Methoden sollen Audiodateien mit Hilfe des ALAudioPlayerModuls abgespielt werden.

\footnotetext{
${ }^{23} \mathrm{https} / / /$ community.aldebaran.com/doc/1-14/naoqi/sensors/alsensors-api.html\#alsensors-events, Zugriff am 22.07.2014
} 


\subsubsection{Teilkonzept 3 - Modul 3 „Geschichte“}

\section{Thematischer Ansatz}

Das folgende Modul wird entwickelt, um auf eine weitere Beeinträchtigung autistischer Kinder einzugehen. In Kapitel 2.4 wurden die Grundzüge der Theory of Mind erläutert. Sie beschreibt die Fähigkeit, eine Annahme über Bewusstseinsvorgänge in anderen Personen vorzunehmen und diese in der eigenen Person zu erkennen, also Gefühle, Bedürfnisse, Ideen, Absichten, Erwartungen und Meinungen zu vermuten [13]. Anders ausgedrückt: Autisten fehlt das soziale Verständnis, wie es dem anderen geht. Durch das nachgewiesen höhere Aufmerksamkeitspotential autistischer Kinder in Anwesenheit des NAO-Roboters und dessen Fähigkeit eine für den Menschen verständliche Sprachausgabe zu verwenden, wird der NAO in diesem Modul dem Kind kurze Geschichten erzählen.

In diesen sogenannten „Social Stories“ spricht er von anderen Kindern, die eine Situation erleben. Aus den Erzählungen soll klar werden, wie die Person in der Geschichte sich fühlt (siehe Kapitel 2.4). Dieses Einfühlungsvermögen, wie es der fremden Person geht, gilt es durch das hier beschriebene Modul zu schulen. Die sozialen Signale werden hierbei durch die Sprache des NAO-Roboters übermittelt. Damit soll das autistische Kind unter anderem die Fähigkeit der Empathie für andere Personen entwickeln und stärken.

Der NAO-Roboter stellt am Ende einer Geschichte folgende Antwortmöglichkeiten zur Auswahl, wie sich die Person in der Situation gefühlt hat:

- traurig,

- glücklich,

- aufgeregt,

- wütend,

- oder ängstlich.

Insgesamt besteht das Repertoire aus fünf Geschichten (siehe Umsetzung in Kapitel 6.3) zu jeweils einem der oben genannten Gefühlsmotive. Das Kind muss dem NAO eines der Gefühle mitteilen. Über die Spracherkennung wertet der Roboter die Antwort aus und lobt es, wenn die Antwort richtig ist. Wenn die Antwort falsch ist, ermuntert der Roboter das Kind es noch einmal zu versuchen. Nach jeder richtig gegebenen Antwort fragt der NAO, ob er eine weitere Geschichte erzählen soll. Dies muss das Kind mit „Ja“ bestätigen. Bei „Nein“ wird das Modul beendet. Der Programmablauf wird in Abbildung 47 deutlich. 


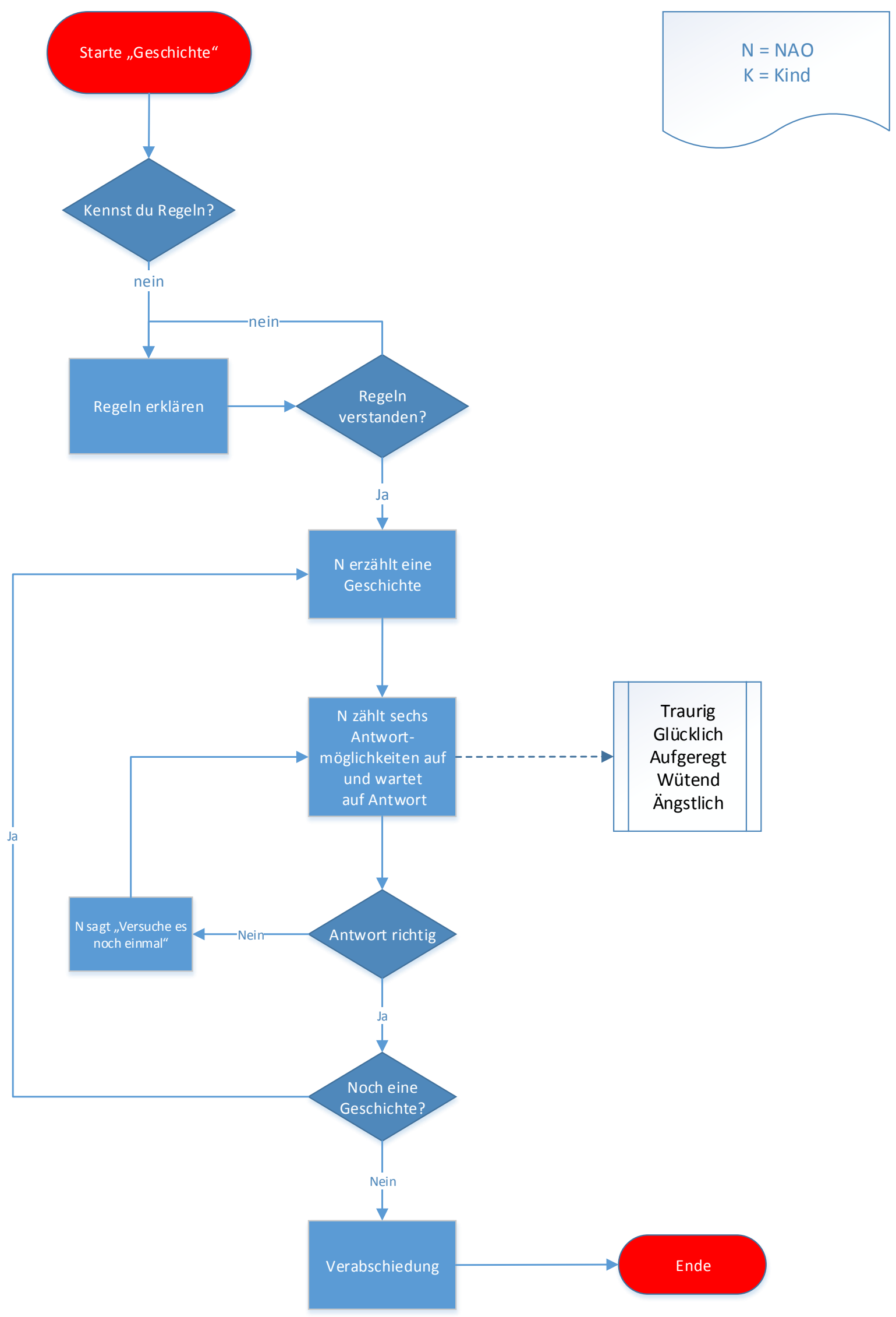

Abbildung 47 - Programmablaufplan Modul 3 "Geschichte" 


\section{Technischer Ansatz}

Tabelle 15 listet zwei weitere Hardwareanforderungen an den NAO auf, die in Modul 3 einen primären Fokus zugeteilt bekommen.

\begin{tabular}{|l|l|l|l|}
\hline Nr. & Aktion des NAO & Fähigkeit & Hardware \\
\hline /M3/HA01/ & Geschichte erzählen & Sprachwiedergabe & Lautsprecher \\
\hline /M3/HA02/ & $\begin{array}{l}\text { Antwort des Kindes } \\
\text { verstehen }\end{array}$ & Spracherkennung & Mikrofone \\
\hline
\end{tabular}

Tabelle 15 - Anforderungen an Modul 3

Für die Sprachwiedergabe (/M3/HA01/) werden wiederum die Lautsprecher des Roboters und für die Spracherkennung (/M3/HA02/) die Mikrofone benötigt. Da in diesem dritten Modul längere Sprachausgaben des NAO-Roboters erfolgen und die Spracherkennung desselbigen mindestens fünf verschiedene Wörter des Kindes verarbeiten muss, werden in diesem Kapitel die ALSpeechRecognition und das NAOqi-eigene ALTextToSpeech-Modul für das weitere Verständnis dargelegt.

\section{ALSpeechRecogniton}

Das Modul ALSpeechRecognition befähigt den NAO, vordefinierte Wörter aus verschiedenen Sprachen zu erkennen. Dafür muss eine Liste mit den zu erkennenden Wörtern als String-Vektor an die Instanz des Moduls übergeben werden. In Falle von Modul 3 werden die Wörter: „traurig“, „glücklich“, „aufgeregt“, „,wütend“ und „ängstlich“ übergeben.

Um eines der Wörter zu erkennen, wird auf den Wert des ausgelösten Events „WordRecognized“ im ALMemory zugegriffen. Dieser Wert kann mit dem erkannten Wort verglichen und im Programmcode ausgewertet werden.

\section{ALTextToSpeech}

Die umfangreiche ALTextToSpeech API ermöglicht dem NAO eine realistische Sprachausgabe. Die ALTextToSpeech-Engine wandelt geschriebenen Text in akustische Signale um, die an die Lautsprecher gesendet werden. Dabei ist es möglich, die Stimme mittels der Parameter „pitchShift“ und „doubleVoice“ individuell anzupassen. Der ,pitchShift"“-Effekt modifiziert die initiale Tonlage der Stimme und der ,doubleVoice“-Effekt produziert ein Echo bzw. eine verzögerte Parallelstimme zur Erststimme. 
Die NAOqi-Dokumentation beschreibt des Weiteren eine Möglichkeit, die Stimme des Roboters zu modifizieren. Die Software ACAPELA (welche als Grundlage für die ALTextToSpeech-Engine dient) synthetisiert ${ }^{24}$ im Hintergrund die Texteingaben als Sprachausgabe. Sie ermöglicht die Verwendung und Auswertung bestimmter tags direkt im Textstring. Tabelle 16 zeigt die wichtigsten tags.

\begin{tabular}{|c|c|c|}
\hline Bezeichnung & tags & Beschreibung \\
\hline Pause & $\mid$ pau=number $\backslash$ & Pause in Millisekunden \\
\hline Geschwindigkeit & $|\mathbf{r s p d}=100|$ & Relative Geschwindigkeit in \%: $50 \% \ldots 200 \%$ \\
\hline Buchstabieren & $\mid r m s=$ value $\backslash$ & $1=$ aktiviert; $0=$ deaktiviert \\
\hline Word für Word & $|r m w=v a l u e|$ & $1=$ aktiviert; $0=$ deaktiviert \\
\hline
\end{tabular}

Tabelle 16 - Auswahl an tags, die die Sprachausgabe manipulieren Quelle: [49]

Des Weiteren können die vom Hersteller angebotenen drei Sprecherstimmen

- Julia22Enhanced

- Sarah22Enhanced

- und Kenny22Enhanced

der Methode setVoice()als Parameter übergeben und an die TextToSpeech-Instanz gebunden werden. Die ausdrucksstärkste und am besten zu verstehende Sprecherstimme wird für die Implementierung der drei Module verwendet.

Im folgenden Kapitel wird nun losgelöst von den obigen drei Teilkonzepten ein Einsatz des Leap Motion Controller diskutiert.

\footnotetext{
${ }^{24}$ Umwandlung von Text in akustische Sprache
} 


\subsection{Der Leap Motion Ansatz}

Der in Kapitel 4.1.6 vorgestellte Leap Motion Controller ist nur bedingt für den Einsatz und die Integration in das Konzept dieser Arbeit nutzbar. Er wird deshalb unabhängig zum restlichen Konzept betrachtet. Um Hand- oder Fingergesten von autistischen Kindern mit dem Controller erkennen zu können, müssten die Kinder unter anderem feinmotorische Voraussetzungen mitbringen. Des Weiteren wäre eine Implementierung eines lokalen Modules in C++ mit dem Controller nicht möglich, da er nicht direkt an den Roboter angeschlossen werden kann. Es käme nur ein Modul per remote in Frage. Zur Programmlaufzeit würde somit ein Notebook als Medium zwischen Controller und dem NAO fungieren. Die Daten zwischen dem Controller und dem NAO würden über WLAN ausgetauscht werden. Der Controller wäre per USB-Kabel am Notebook angeschlossen und müsste genau vor dem Kind liegen. Es müsste die Hände immer über dem Gerät halten, damit die Interaktion der Hände erkannt wird (Abbildung 48).

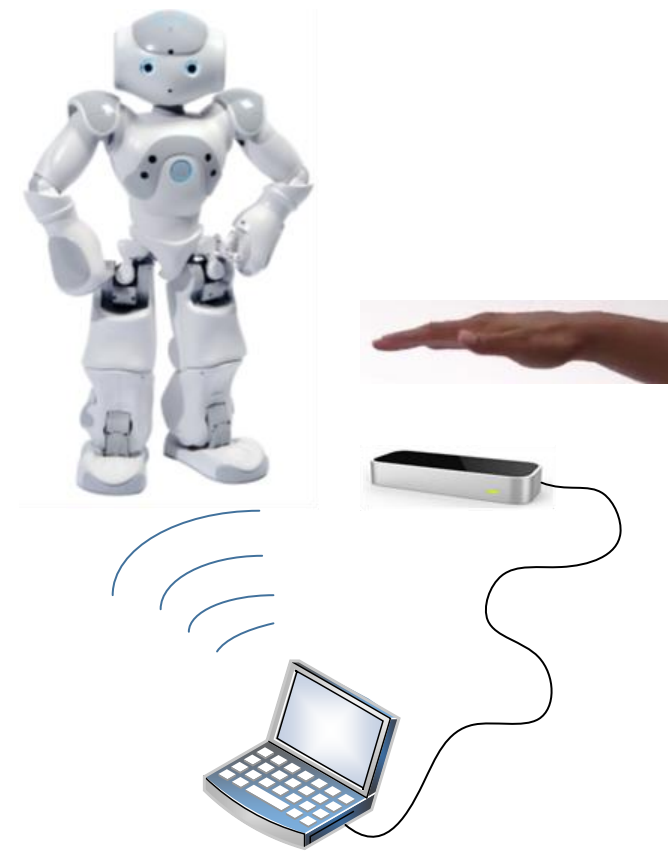

Abbildung 48 - Aufbau einer Leap Motion-Sitzung mittels eines Notebooks

Um im Ansatz eine Einsatzmöglichkeit zu zeigen, wurde eine Idee entwickelt, wie die Zahlen von 0 bis 10 mit den Fingern geübt werden können. Dafür ist das Verständnis der autistischen Kinder über die Anzahl der eigenen Finger nötig. Der NAO könnte in einer Sitzung das Kind bitten, eine bestimmte Anzahl an Fingern zu zeigen. Diese müsste das Kind dann genau über den Controller halten. Wenn die richtige Anzahl an Fingern gezeigt wird, spricht der NAO ein Lob aus. Um beim Abschied oder bei der Begrüßung eine „Wink“-Bewegung zu erkennen, könnte die vordefinierte Handgeste onSwipe() ausgewertet werden. Dafür müsste genau 
über dem Controller gewinkt werden. In folgender Grafik wird der mögliche Konzeptansatz dargestellt.

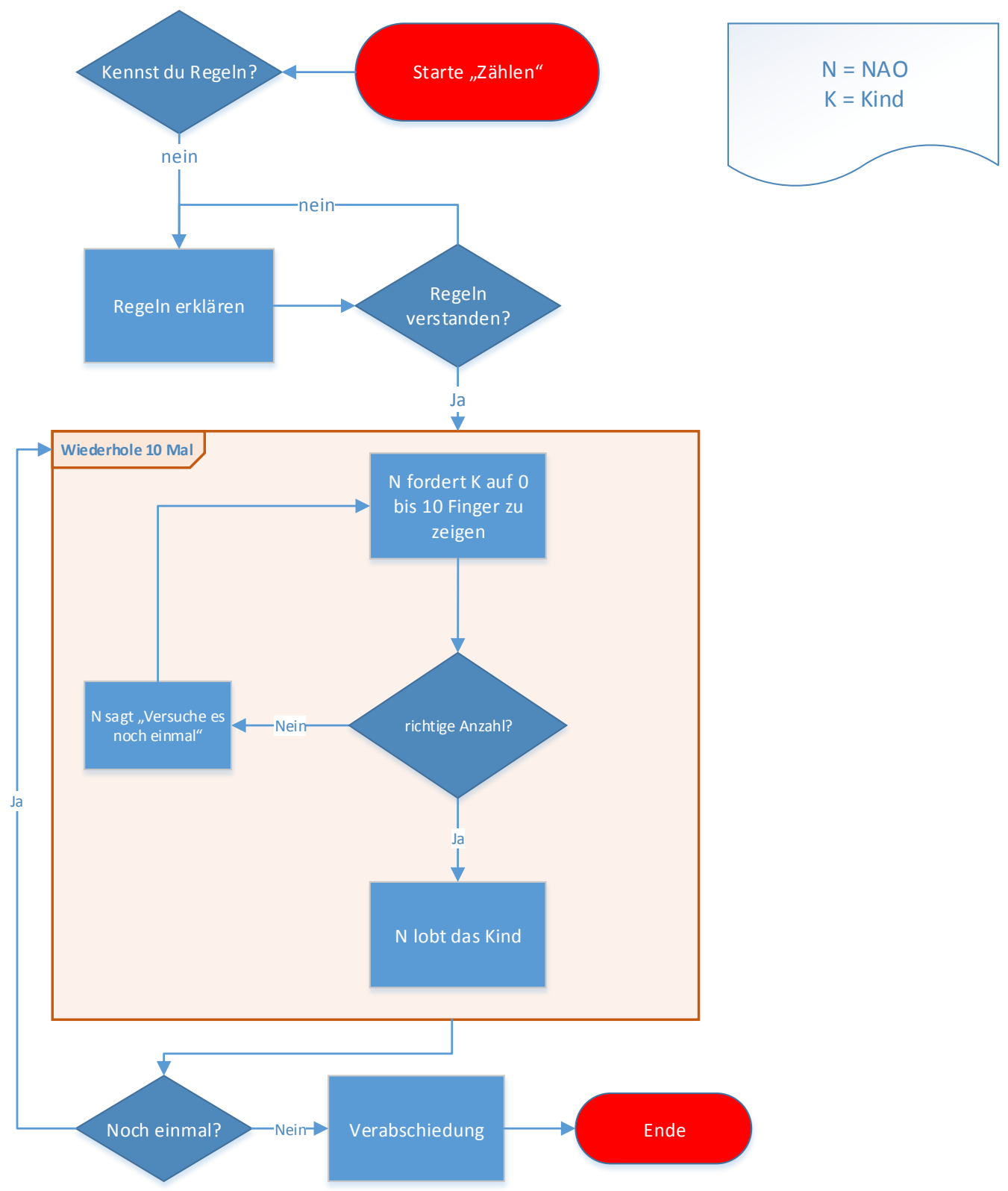

Abbildung 49- Programmablaufplan für Modul mit Leap Motion Controller

Eine Human-Robot-Interaktion mit dem Leap Motion Controller zu realisieren ist denkbar. Unter der Betrachtung der eingeschränkten Fähigkeiten eines autistischen Kindes, wie z.B. das stereotyp repetitive Verhalten seine Hände nicht kontrollieren zu können oder fehlender motorischer Fähigkeiten, wird von einem Einsatz und einer Umsetzung in dieser Arbeit abgesehen. Der Einsatz des Controllers bietet deutlich mehr Möglichkeiten für Personen mit einer altersgerechten Entwicklung und keinen Einschränkungen aus dem autistischen Spektrum. 


\section{Umsetzung}

Im Kapitel der Umsetzung wird auf wichtige Aspekte und Ergebnisse der programmiertechnischen Implementierung der Module eingegangen. Dabei wird ersichtlich, welche Konzeptionsziele der Autor umgesetzt hat. Im ersten Abschnitt 6.1 werden die Ergebnisse der praktischen Testreihe mit einem autistischen Kind beschrieben. Darin wird auf die Entwicklung der dabei entstanden beiden Module „Berühre Mich“ und die englische Version „Touch Me“ eingegangen. Die Umsetzung der Module „Gefühle“ und „Geschichte“ wird im Abschnitt 6.2 und 6.3 beschrieben.

\subsection{Praktische Studie}

Die Professorin Janett Mohnke der TH Wildau stellte während des Bearbeitungszeitraums einen Kontakt zur Ambulanzlehrerin Kerstin Michlo in Berlin her. Frau Michlo, welche für den Autismus Deutschland Landesverband Berlin e.V. arbeitet, vermittelte ein Treffen mit den Betreuern der Ganztags-Kleinklasse I im Frühförderzentrum ${ }^{25}$ für autistische Kinder in Berlin. Nach einem Vorgespräch mit Frau Michlo wählten die Betreuer ein nach ihren Einschätzungen geeignetes Kind aus, welches für eine Testreihe mit dem NAO-Roboter in Frage käme. Insgesamt konnten vier Treffen mit Lino $^{26}$ und Theo ${ }^{27}$ im Frühförderzentrum arrangiert werden. Dazu wurde ein Raum gewählt, der den Bedingungen des Raumsetup in Kapitel 5.1 entsprach.

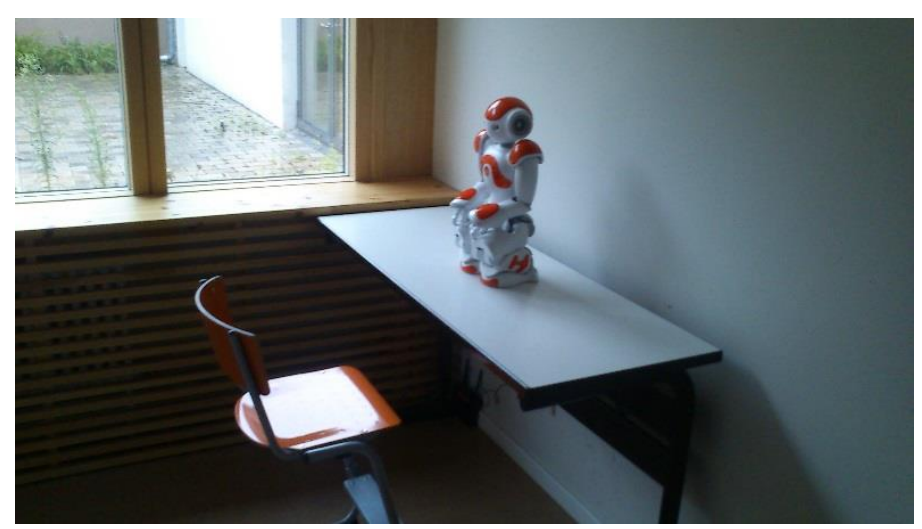

Abbildung 50- Testraum im Frühförderzentrum in Berlin Quelle: eigenes Foto

Vorab wird in Stichpunkten die Persönlichkeit Theos beschrieben. Dabei werden nur die Aspekte aufgezählt, die für das weitere Verständnis nötig sind. Die zusammengestellten Angaben wurden von Theos Bezugsbetreuerin Esther Grohmann zur Verfügung gestellt.

\footnotetext{
${ }^{25}$ Marchlewskistraße 25d in Berlin-Friedrichshain

${ }^{26}$ Der Name wurde dem NAO gegeben, damit das Kind einen Bezug zu ihm aufbauen kann.

${ }^{27}$ Der Name wurde aus Datenschutzgründen vom Autor geändert.
} 
- Alter: 9 Jahre

- türkische Abstammung

- $\quad$ seit drei Jahren in der Einrichtung

- Hält zwanghaft an vertrauten Abläufen fest.

- Spielt gern mit Autos und Computern.

- Ahmt z.Z. gern besonderes Verhalten von Mitschülern nach.

- Hat große Freude daran, Reaktionen bei Mitschülern hervorzurufen.

- Erfüllen von Unterrichtsaufgaben fällt ihm meist schwer, entweder langweilen ihn die Aufgaben oder er versteht die Arbeitsaufgabe nur unzureichend.

- Wenn Theo die Aufgabe verstanden hat und sie seinen Kompetenzen entspricht, kann er schnell arbeiten.

- Ihm fällt es leicht, Muster zu durchschauen und Kategorien zu bilden.

- Theo zu motivieren ist schwierig, da er abgesehen von Computerspielen, Handys etc. wenig Interessen und Vorlieben hat.

Bisherige Entwicklung der Kommunikation:

- $\quad$ sprach nach den ersten 2 Schuljahren keine deutschen Worte, wiederholte stereotyp Worte und Satzstücke aus amerikanischen Trickfilmen und Computerspielen

- hatte nicht näher differenzierbaren passiven Wortschatz (ob er Sätze nicht verstand oder dem nicht nachkommen wollte, war nicht immer ersichtlich)

- spricht zu Hause nicht, weder türkisch noch deutsch, nur Sätze in Englisch (aus Trickfilmen etc.)

- begann im dritten Schuljahr zunächst, wenige deutsche Worte nachzusprechen und später von sich aus zu verwenden, dies beschränkt sich hauptsächlich auf Äußerungen, die ihm sehr vertraut sind und häufig wiederkehren (Begrüßung, Verabschiedung, "Ich möchte..."-Äußerungen, Begrüßung im Morgenkreis)

In den folgenden vier Tests wird die individuelle Anpassung und Umstrukturierung des in Kapitel 5.1.2 entwickelten „Berühre Mich“-Moduls anhand der Testverläufe und der Testergebnisse beschrieben. Für die Auswertung des Tests wurde die eigens für den Erstkontakt erstelle Wertungsmatrix (Kapitel 5.1, Tabelle 7) verwendet. Die vier Bewertungsbögen der Tests werden, um Lesefluss nicht nachteilig zu beeinflussen, im Anhang A.3.-A.6. angefügt. 


\subsection{1. $\quad$ Test 1}

Angelehnt an die Studie von S. Shamsuddin [4], in der die initiale Reaktionsbereitschaft autistischer Kinder gegenüber dem NAO getestet wurde, sind für die erste Begegnung mit Theo vom Autor einige Behaviors mit Choregraphe erstellt worden, um die Reaktion auf verschiedene Aktionen des Roboters zu testen und um herauszufinden, welches der drei Module als Therapieansatz eingesetzt werden soll. Die einzelnen in Abbildung 51 dargestellten Boxen wurden nacheinander ausgeführt und die Reaktion von Theo beobachtet. $\checkmark$ 立root \begin{tabular}{|l|l|l|l|l|l|l}
\hline 1. Test Theo \\
\hline
\end{tabular}

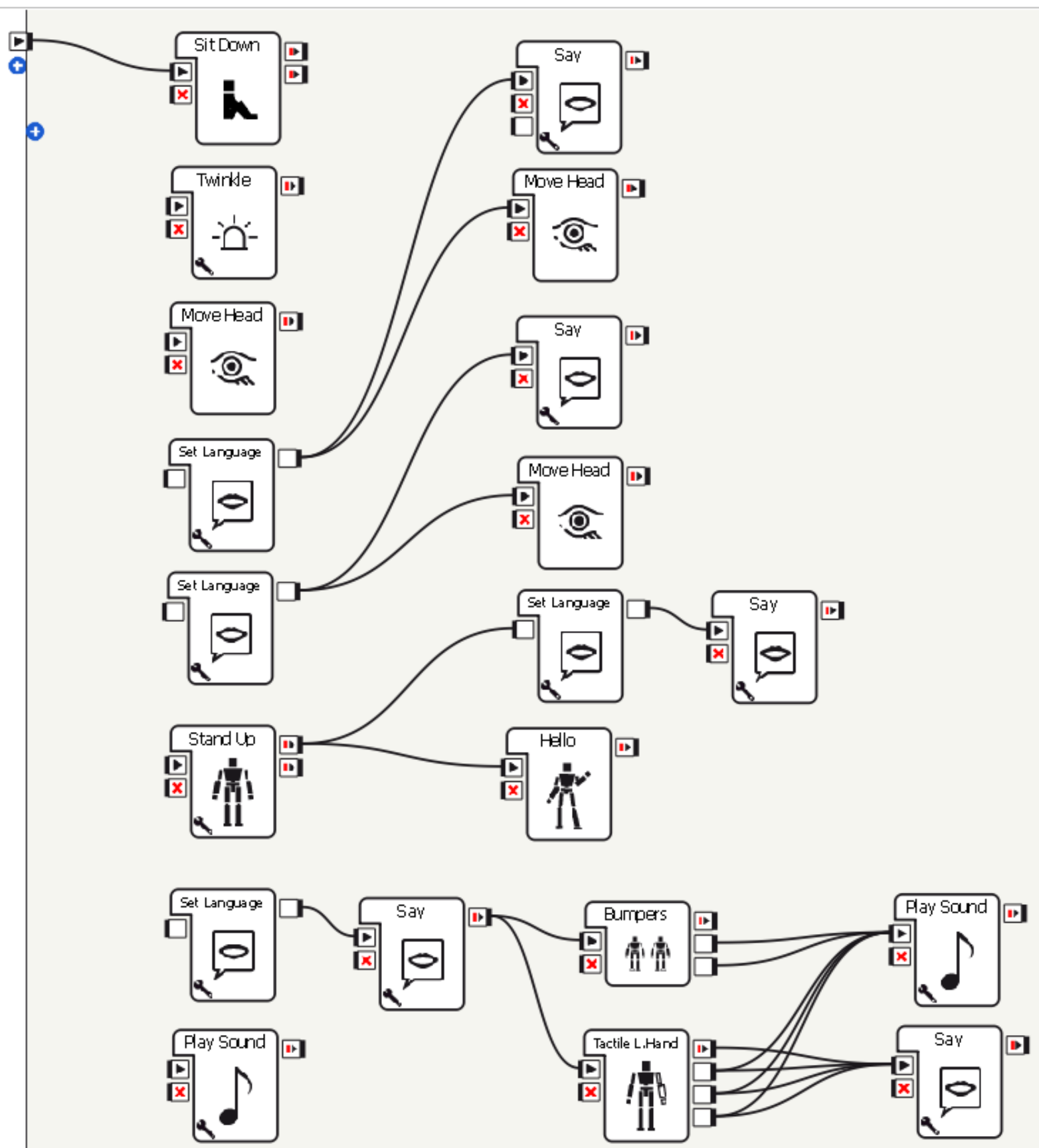

Abbildung 51 - Behaviors für den Erstkontakt, erstellt mit Choregraphe 
Die Erklärungen der Behaviors in Abb. 52 werden wie folgt beschrieben:

$\begin{array}{ll}\text { „Sit Down“ } & \text { Lino sitzt auf dem Tisch. } \\ \text { „Twinkle“ } & \text { Lino blinkt mit den Augen. } \\ \text { „Move Head“ } & \text { Lino bewegt den Kopf mehrmals nach rechts und links. } \\ \text { „Set Language“ } & \text { Lino spricht auf Deutsch: , Ist dort jemand? “. } \\ \text { „Set Language“ } & \text { Lino spricht auf Englisch: „, Is anybody there? “. } \\ \text { „Stand Up“ } & \text { Lino steht aus der Sitzposition auf, winkt im Stehen während er } \\ & \text { auf Deutsch „Hallo“ sagt. } \\ \text { „Play Sound“ } & \text { Lino spielt eine Musikdatei }{ }^{28} \text { über die Lautsprecher ab. } \\ \text { „Bumpers“ } & \text { Lino spielt bei der Berührung eines Fußsensors eine kurze } \\ & \text { Melodie ab. }\end{array}$

Um die Behaviors auf dem NAO-Roboter auszuführen, musste sich dieser im selben Netzwerk wie das Notebook befinden, auf dem Choregraphe ausgeführt wird. Da beim ersten Test kein WLAN-Router zur Verfügung stand, wurde ein Tethering-Hotspot auf dem NAO eingerichtet. Dazu musste das Notebook zuerst per Netzwerkkabel mit der Netzwerkbuchse des Roboters verbunden werden. Danach konnte über das Webinterface der Hotspot auf dem NAO aktiviert werden. Eine Kabelverbindung zwischen dem Roboter und dem Notebook während einer Sitzung, hätte unter Umständen die Bewegungsfreiheit des Roboters eingeschränkt.

\section{Auswertung}

Die Auswertung des Bewertungsbogens zeigt, dass Theo generell interessiert auf die Präsenz des Roboters reagierte, als dieser still auf dem Tisch saßt. Er ging sofort hin und berührte ihn. Die Kopfbewegung und das Blinken der Augen zeigten keine Reaktion. Bis auf die Berührung des Fußes zeigte Theo mäßiges Interesse. Die auffälligste Reaktion zeigte Theo bei der Berührung des Fußsensors. Sobald er realisierte, dass die Berührung das Abspielen einer Melodie auslöst, betätigte er von alleine mehrmals den Sensor. Auf Sprachausgaben des Roboters zeigte er keine Reaktion.

\footnotetext{
${ }^{28}$ Hier wurde das Kinderlied „Hänschen klein, ging allein“ ausgewählt. 
Zusammen mit der Bezugsbetreuerin von Theo wurde auf Grund der eindeutigen Reaktion auf die Fußsensoren das Modul 2 „Berühre Mich“ als Grundlage für weitere Test ausgewählt. Da von den Therapeuten parallel eine Förderung der Kommunikation angestrebt wird und Berührungen ein Schlüsselaspekt der Kommunikationsfähigkeit sind, stellte die Auswahl des Moduls eine optimale Fördergrundlage dar.

\subsection{2. $\quad$ Test 2}

In Absprache mit Frau Grohmann, wurde das Modul 2 dahingehend modifiziert, dass den verschiedenen Sensoren Audiodateien hinterlegt wurden, die Theo aus dem Alltag bekannt sind. In Abbildung 52 werden fünf Sounddateien als feste Konstanten definiert.

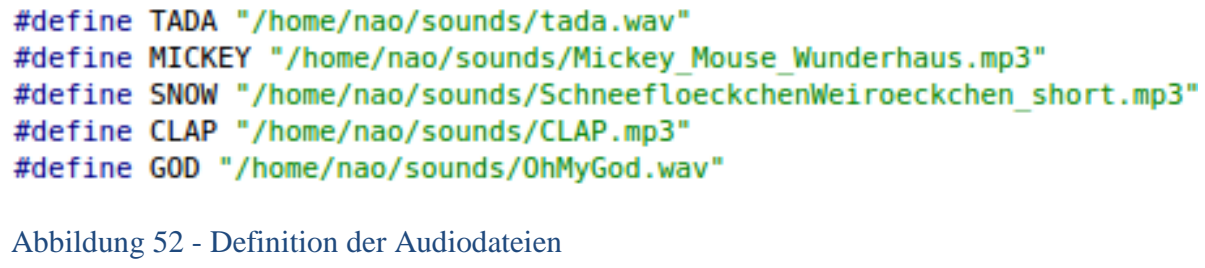

Des Weiteren wurde eine persönliche Begrüßung für Theo an den Beginn des Moduls gesetzt. Während der Begrüßung winkt Lino mit dem Arm.

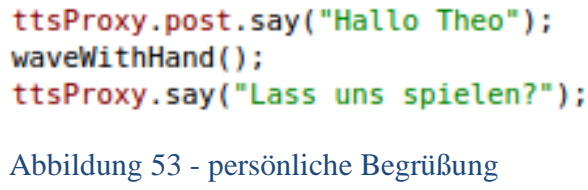

Die im Ausgangsmodul implementierten zehn Sensoren (Auflistung in Kapitel 5.1.2) wurden auf sechs reduziert, da die jeweiligen Handseiten in der Sitzposition schlecht zu erreichen sind und die Auslösegenauigkeit keine brauchbare Handhabung ermöglicht. Somit werden noch folgende Sensoren ausgewertet.

Fußspitzen:

- RightBumperPressed (rechter Fuß)

- LeftBumperPressed (linker Fuß)

Kopfsensoren:

- FrontTactilTouched (Stirn)

- RearTactiltouched (Hinterkopf)

Rechte Hand:

- HandRightBackTouched (Handrücken)

Linke Hand:

- HandLeftBackTouched (Handrücken) 
Des Weiteren wurden die Sätze und Wörter, die Lino von sich gibt, vereinfacht. Das Hauptziel bestand nun darin, dass Theo den Aufforderungen Linos von alleine nachkommen sollte. Durch die bekannten Melodien sollten die Berührungsängste gemindert und eine persönliche Bindung aufgebaut werden. Für die Sprachausgabe wurde die Stimme Julia22Enhanced ausgewählt. Sie verleiht Lino eine weiche und eher kindliche Stimme.

Das Modul „Berühre Mich“ wurde wie in der Konzeptübersicht zusammen mit dem Moderator-Modul auf dem NAO ausgeführt. Um das Modul 2 zu starten, waren Spracheingaben nötig, die die Betreuerin übernommen hat.

\section{Auswertung}

Beim zweiten Test war eine andere Betreuerin mit dabei. Das Modul „Berühre Mich“ wurde zweimal ausgeführt. Wie dem Auswertungsbogen in Anlage A3 zu entnehmen ist, hat Theo nur auf die Aufforderungen von Lino reagiert, wenn der Betreuer die Aufforderung wiederholte und zusätzlich Theos Hand nahm und sie zu dem gefragten Körperteil führte (siehe gestützte Kommunikation*). Beim Abspielen der bekannten Melodien wirkte er interessiert und lächelte. Während der zweiten Spielrunde legte er sich auf den Tisch und wirkte etwas abwesend. Theo hat während der Sitzung mit Lino keinen Blickkontakt hergestellt oder gehalten.

\subsection{3. $\quad$ Test 3}

Da Theo laut den Angaben aus seinem Persönlichkeitsprofil auch Englisch verstehen kann, wurde dasselbe Modul als englische Variante implementiert, in welcher die Programmlogik erhalten blieb und alle Sprachausgaben auf Englisch getätigt wurden. Zudem wurde zusätzlich zu jeder Aufforderung eine Bewegung des entsprechenden Körperteils implementiert. Das heißt, Lino streckt Theo den Fuß oder die Hand entgegen. Wenn er den Kopf berühren soll, neigt er diesen nach vorne.

Um die jeweiligen Bewegungen zu realisieren, wurde der Animationsmodus der Choregraphe Suite verwendet. Mit ihm sind die einzelnen Motions erstellt und dann in C++ Quellcode exportiert worden (Anlage A.1.). Weiterhin wurde zusätzlich eine moves.cpp erstellt, um die Übersicht im Quellcode zu erhalten. Jede erstellte Bewegung wurde in einer eigenen Methode innerhalb der moves. cpp untergebracht. Die Methoden werden direkt hinter der passenden verbalen Aufforderung der entsprechenden Körperstelle im Programmablauf aufgerufen. Siehe Abbildung 54: 


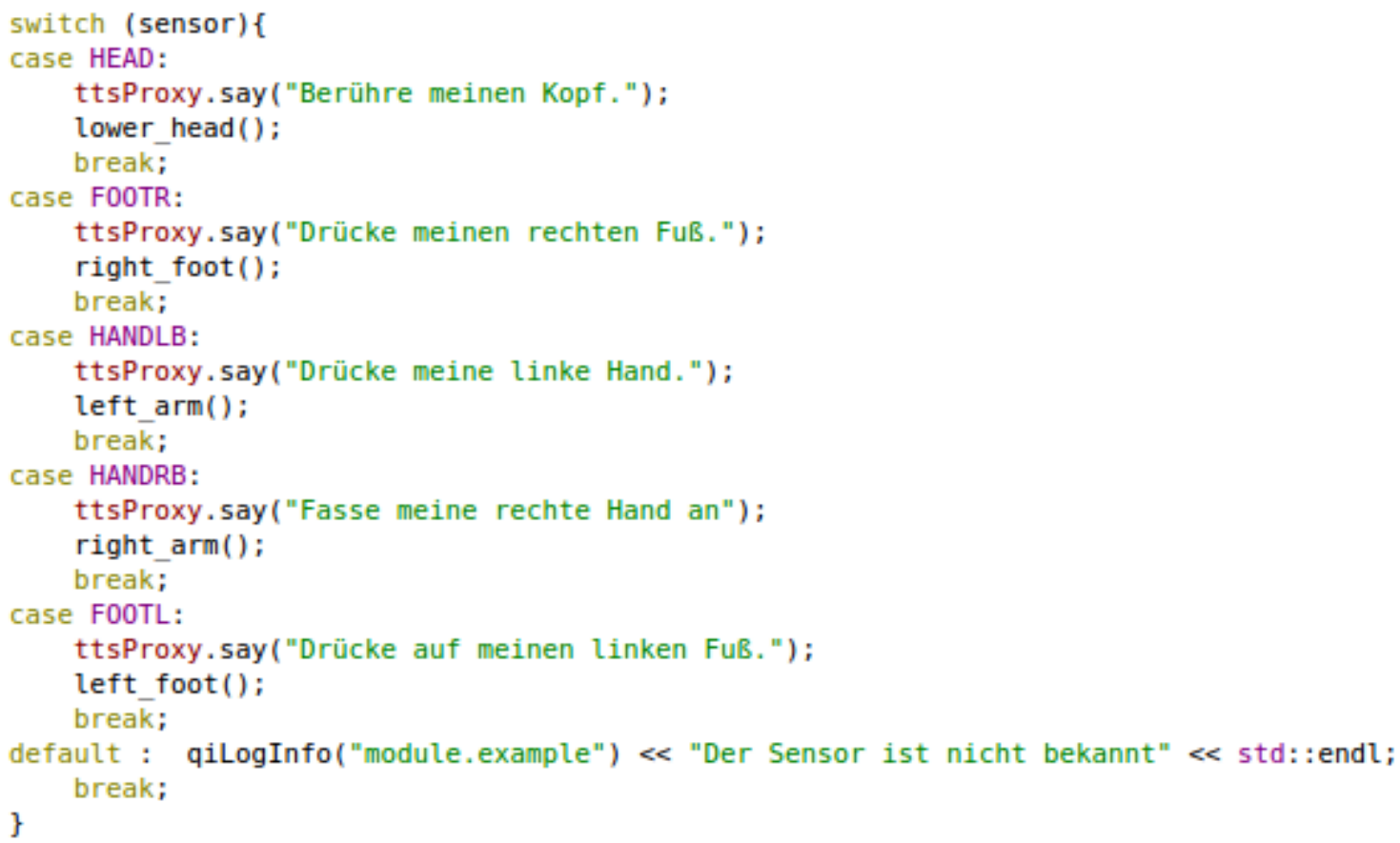

Die zugehörigen Bewegungsänderungen werden in der der folgenden Grafik anhand der Methoden left_foot () und left_Hand () visualisiert dargestellt.
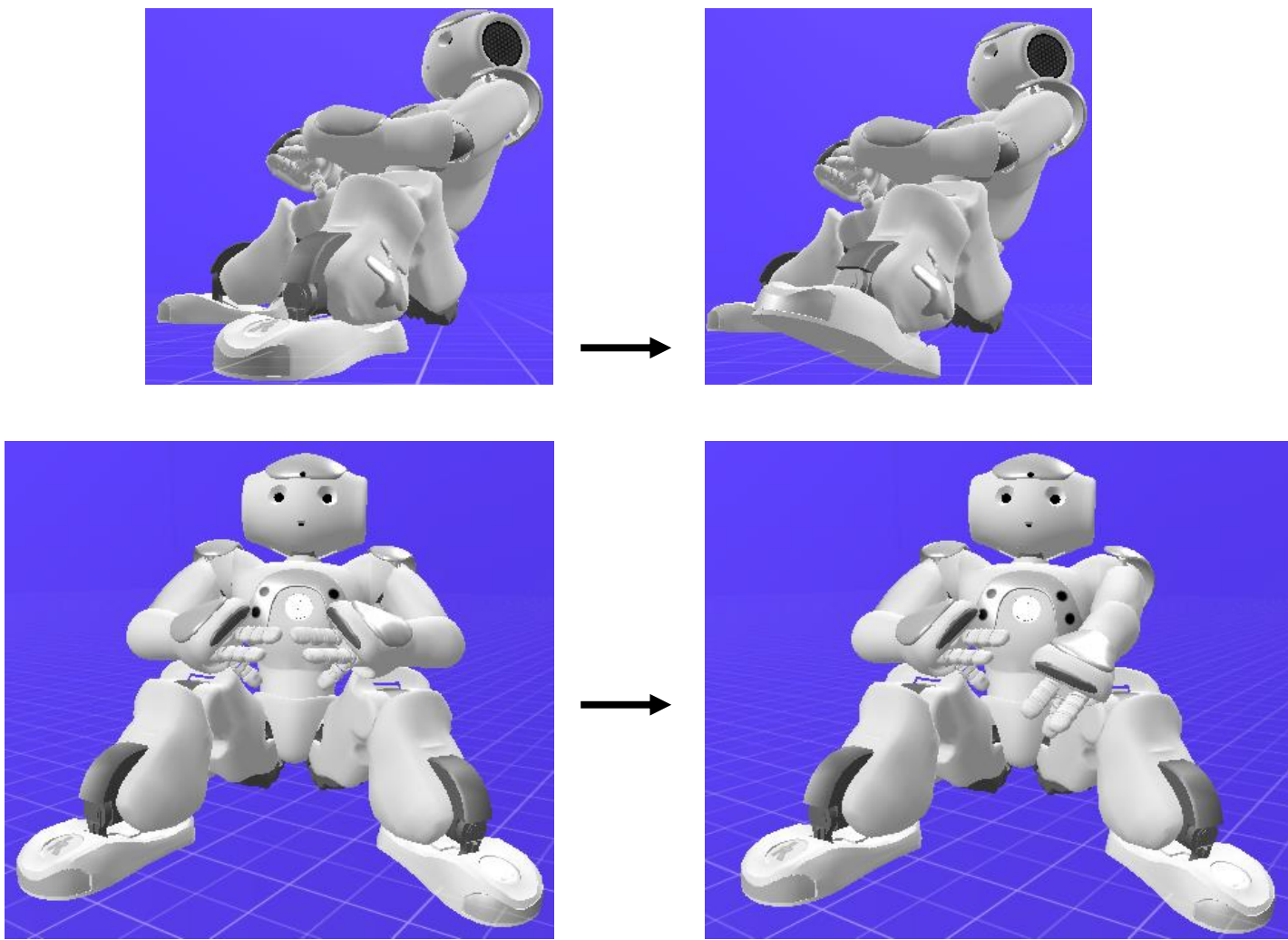

Abbildung 55 - Bewegungsänderung der Fuß- und Armgelenke 


\section{Auswertung}

Bei diesem Test war Theos Bezugsbetreuerin, Frau Grohmann, wieder mit dabei. Das Modul wurde mit den implementierten Änderungen einmal auf Deutsch und einmal auf Englisch abgespielt. Theo wirkte an diesem Tag müde. Er kam wiederum den Aufforderung des Roboters nicht ohne weitere Anweisung der Bezugsbetreuerin nach. Dafür zeigte Theo Interesse, wenn der Roboter seinen Fuß oder seinen Kopf bewegte. Durch die langjährige therapeutische Beziehung zu Frau Grohmann reagierte Theo bei diesem Test fast ausschließlich auf die verbale Ansprache ihrerseits und benötigte keine Führung seines Armes zu der entsprechenden Körperstelle des Roboters. Beim zweiten Durchlauf klatschte Theo beim Abspielen eines bekannten Audiofiles mehrmals in die Hände. Zusätzlich lässt sich sagen, dass eine erhöhte Konzentration zu verzeichnen und ein häufiger Blickkontakt in der Videoaufnahme erkennbar war.

\subsubsection{Test 4}

Da der linke Handrückensensor im vorangegangen Test 2-3 Mal recht schwerfällig auslöste, wurde der kapazitive Sensor unterhalb der Handverschalung ein wenig angehoben, um die Berührungsempfindlichkeit zu erhöhen.

Die Unterteilung in Stirn und Hinterkopf führte bei der Berührung durch Theo oft zu Fehlberührungen. Wenn er den Hinterkopf berühren sollte, kam er oft zuerst an die Stirn. Ausgehend von dieser Tatsache wurde die Anzahl der Körperstellen ein weiteres Mal auf fünf gemindert und die Berührung für den gesamten Kopf vereinfacht. Das heißt, dass die Auslösung der drei Kopfsensoren FrontTactilTouched, MiddleTactilTouched und RearTactilTouched jeweils als korrekte Berührung des Kopfes ausgewertet wurde. Lino forderte Theo nun nur auf, seinen Kopf zu berühren. Programmiertechnisch musste dafür zusätzlich das MiddleTactilTouched-Event ausgewertet werden.

Das Modul wurde wieder einmal auf Deutsch und einmal auf Englisch abgespielt.

\section{Auswertung}

Zur Auswertung des vierten und letzten Tests ist $\mathrm{zu}$ bemerken, dass die Ergebnisse die Erwartungen übertrafen. Die Bezugsbetreuerin war wieder dabei. Es wurde einmal das englische Modul und einmal das deutsche Modul gespielt. Die gesamt Spieldauer betrug ca. acht Minuten (4 min. Deutsch, 4 min Englisch). 
Theo setzte sich von allein auf dem Stuhl vor Lino und saß ganz aufrecht und gespannt da. Aus den Videoaufnahmen und Beobachtungen während der Sitzung war eine auffällig höhere Konzentration zu sehen. Theo hielt innerhalb eines Spielablaufes insgesamt ca. 60 Sekunden Augenkontakt mit Lino ${ }^{29}$. Des Weiteren kam Theo den Aufforderungen Linos fünf von zehn Mal selbständig nach und berührte die entsprechende Körperstelle. Viel mehr reagiert er damit auf die Bewegung des Roboters als auf die verbale Bitte. Die Bewegungen konnte Theo nur sehen, wenn er nicht zur Seite schaute und Blickkontakt hielt. Nichtdestotrotz zeigt sich damit, dass Theo seine Berührungsängste zu Lino gemindert hat und ihm die Situation mit dem Roboter nicht mehr fremd ist. Wie an den markierten Feldern im Bewertungsbogen des vierten Tests in Anlage A.7. zu sehen ist, war nur fünf Mal eine Unterstützung durch die Bezugsbetreuerin nötig. Überraschend sprach Theo das erste Mal seit dem Kontakt mit Lino. Als der Roboter zum Abschied winkte und „Auf Wiedersehen“ sagte, antwortete Theo mit einem eindeutigen ,Tschüss“.

Eindeutig lässt sich nach dem vierten Test anhand der Bewertungsbögen und der Beobachtungen eine Tendenz nachweisen, die positiv für die Entwicklung der Beziehung zwischen dem autistischen Kind Theo und dem Roboter spricht. In folgenden Förderschwerpunkten konnten positive Ergebnisse nachgewiesen werden:

- erhöhte Aufmerksamkeit

- häufiger Blickkontakt

- gemindertes stereotypes Verhalten

- geminderte Berührungsangst

- erhöhtes Interesse an der Interaktion mit dem Roboter

- soziale Signale deuten (entgegengestreckte Hand/Fuß berühren)

Das Hauptziel, dass Theo den Aufforderungen Linos nachkommt, wurde mit Hilfe der gestützten Kommunikation erreicht. Die gebärdenhafte Gestik des Roboters dient als Brücke zur verbalen Kommunikation. Als weiterführendes Ziel wird die sukzessive Zurücknahme der einzelnen Bewegungen empfohlen, um den Fokus auf das Verständnis der verbal getätigten Sprachanweisungen des Roboters zu legen.

Im Abbildung 56 auf der folgenden Seite wird der Programmablaufplan des für Theo abgeänderten und angepassten Moduls 2 „Berühre Mich“ grafisch visualisiert. Der PAP zeigt

\footnotetext{
${ }^{29}$ Die angegebene Zeit wurde anhand der Videoauswertung empirisch vom Autor dieser Arbeit gemessen.
} 
das Ergebnis der Umsetzung nach dem 4. Test. Der Quellcode des Moduls ist auf der beiliegenden DVD zu finden.

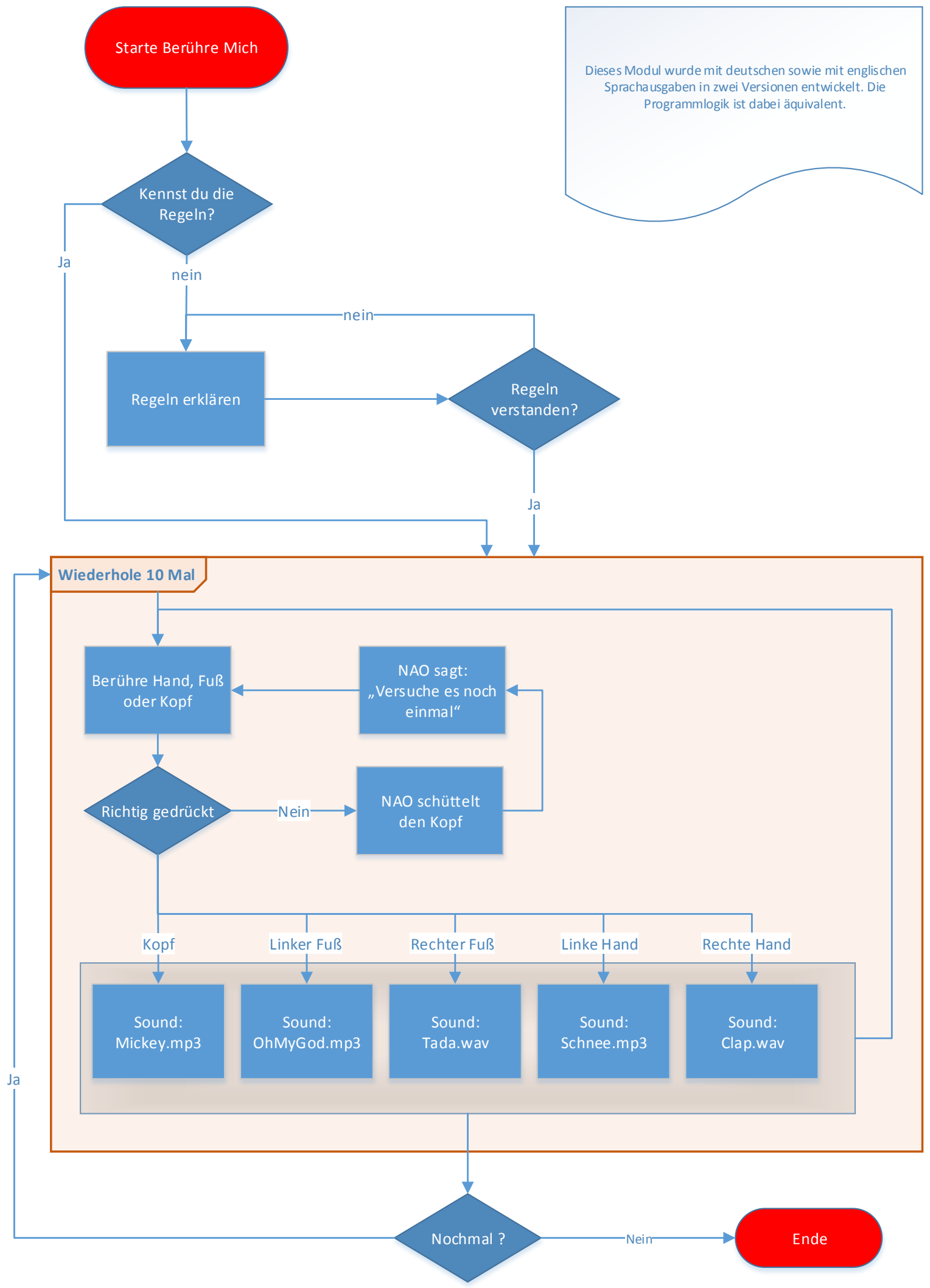

Abbildung 56 - Programmablaufplan Modul 2 „Berühre Mich“, angepasste Version 


\subsection{Modul „Gefühle“}

Das Modul „Gefühle“ wurde wie im Teilkonzept 1 (Kapitel 5.1.1.) beschrieben vollständig umgesetzt. Im Folgenden wird auf einige Besonderheiten bei der Umsetzung eingegangen. Der Quellcode und eine ausführliche Doxygen*-Dokumentation befinden sich auf der DVDBeigabe.

Für die Umsetzung des Moduls wurden zuerst die Emotionen „wütend“, „traurig“ und „glücklich“ mit dem Animationsmodus in Choregraphe erstellt. Dabei wurde wie in Anlage A.1. beschrieben vorgegangen. Mittels qibuild und dem QTCreator wurde innerhalb der virtuellen Maschine ein neues Projekt mit dem Namen THW_Game_Imitate erstellt. Folgende Abbildung 57 zeigt die Ordnerstruktur des Projektes:

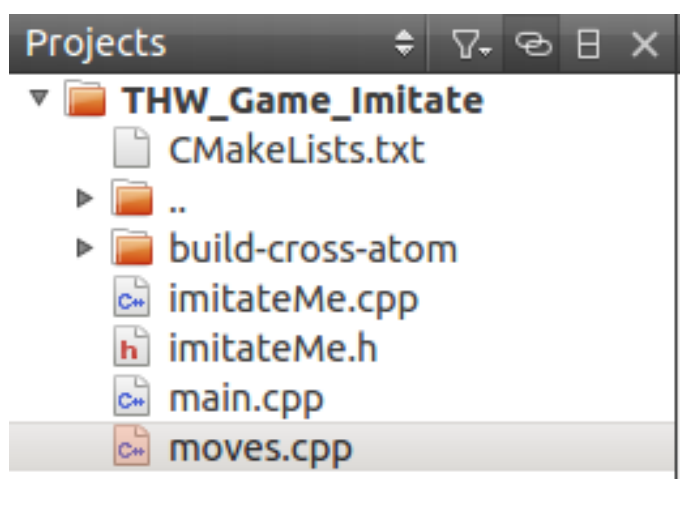

Abbildung 57 - Projektstruktur des Moduls „Gefühle“

Die moves. cpp enthält die exportierten Motions, welche in jeweils einer eigenen Methode gespeichert und in der imitateMe.h bekannt gemacht wurden. Die imitateMe.cpp enthält die Programmlogik. Die main.cpp wird automatisch vom QTCreator erstellt und dient wie auch die CMakeList.txt der Erstellung eines lokalen Moduls. Für eine ausführliche Beschreibung zur Erstellung von lokalen Modulen wird auf das Tutorial von Georg Labbé verwiesen (siehe DVD-Beigabe).

Für die Worterkennung wurden zusätzlich zu ,ja“ und „,nein“ die Wörter „wütend“, „traurig“ und ,glücklich“ der Wortliste hinzugefügt:

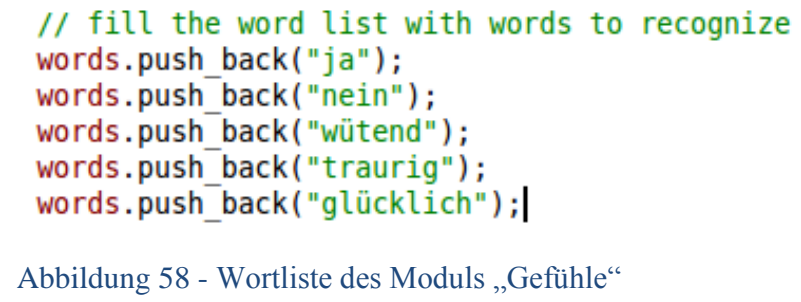


$\mathrm{Zu}$ Beginn des Moduls zeigt Lino alle drei Emotionen nacheinander. Dabei sollte er jede Emotion zeitgleich mit der Bewegung verbalisieren und eine entsprechende Stimmung mit den Augen erzeugen. Dabei trat in der C++-Implementierung folgendes Problem auf: Jede TextTospeech-Anweisung wird als blockierende Methode ausgeführt. Jede angleInterpolationBezier( ) -Methode, die für die Bewegungsausführung zuständig ist, blockiert ebenfalls, solange sie ausgeführt wird. Das Gleiche gilt für die Methode fadeRGB(), die für die Änderung der Augenfarbe zuständig ist. Hierfür musste eine Lösung gefunden werden, wie die Sprache, die Bewegung und die Änderung der Augenfarbe parallelisiert werden können.

Die NAOqi-API der Version 1.14.5 bietet dafür eine Lösung. Jeder erstellte Proxy besitzt das Attribut post, welches genutzt werden kann, um längere Methoden in einem parallelen Task zu starten. Dafür muss vor die jeweilige aufzurufende Methode das Präfix post gesetzt werden.

ttsProxy.post.say("ich bin soo traurig.");

motion_sad ( );

Abbildung 59 - Quellcodeausschnitt, imitateMe.cpp

In obigem Quellcodeausschnitt wird zwischen dem TextToSpeech-Proxy und der Methode say() das Schlüsselwort post eingesetzt, um die Sprachanweisung im Hintergrund auszuführen. Danach folgt die Methode für die Bewegungsausführung, welche in der moves.cpp hinterlegt ist. Im unteren Quellcodeausschnitt wird innerhalb der Methode motion_sad() die Parallelisierung der Farbänderung der Augen und der Bewegungsausführung deutlich.

eyeleds.post. fadeRGB ("Faceleds", 0xө0FFө000, 3);

motion.angleInterpolationBezier(names, times, keys);

Abbildung 60 - Quellcodeausschnitt, moves.cpp Methode: motion:sad()

Der erste Parameter der fadeRGB ( ) -Methode gibt an, welche LED angesteuert werden, der zweite gibt den Farbwert in HEX an. Der dritte Parameter gibt die Zeit in Sekunden an, bis die Farbintensität zu $100 \%$ erreicht ist.

Mit dieser Lösung war es möglich, die Verbalisierung und die Bewegung annähernd zeitgleich auf dem NAO-Roboter auszuführen. Mit der zur Zeit der Ausarbeitung noch nicht zur Verfügung stehenden NAOqi Version 2.1 sollen weitere Audio-Bibliotheken hinzugefügt werden. Mit der dann verfügbaren ALAnimatedSpeech API z.B. soll es möglich werden, 
Sprache und passende Körperbewegungen zeitgleich auszuführen und gezielt aufeinander anzupassen [51].

Um Lino während der Emotion „glücklich“ mit den Augen zwinkern zu lassen, wurde folgende Methode entwickelt:

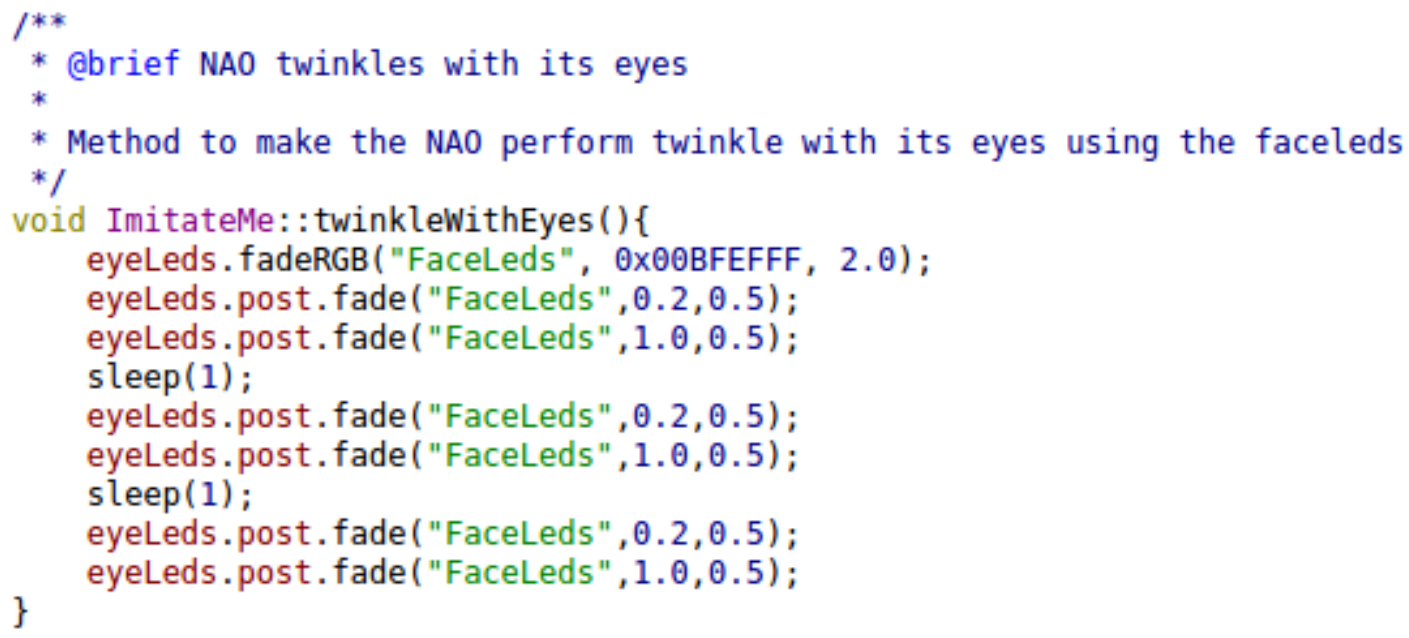

Abbildung 61 - Quellcodeausschnitt, imitate.cpp

Die erste Anweisung der Methode legt die Augenfarbe mit dem Wert 0x00BFEFF fest. Danach wird im Abstand von einer Sekunde die Farbintensität innerhalb von 0.5 Sekunden auf $20 \%$ und gleich im Anschluss wieder auf $100 \%$ gesetzt. Das erzielt den Effekt, als ob der Roboter zwinkern würde.

Im letzten Drittel des Moduls fordert der Roboter dazu auf, dass seine Bewegungen vom Betreuer oder Kind nachgemacht werden. Um zu kontrollieren, ob sich die Personen auch bewegen, sollte das Event MovementDetection/MovementDetected ausgewertet werden. Mittels der Kopfkamera und dieser vom Hersteller zur Verfügung gestellten API ist es möglich Bewegungen vor dem NAO zu erkennen und auszuwerten. Mit folgender Codezeile lässt sich das Event am ALMemoryProxy registrieren. Die Pfeile erklären die Anweisung.

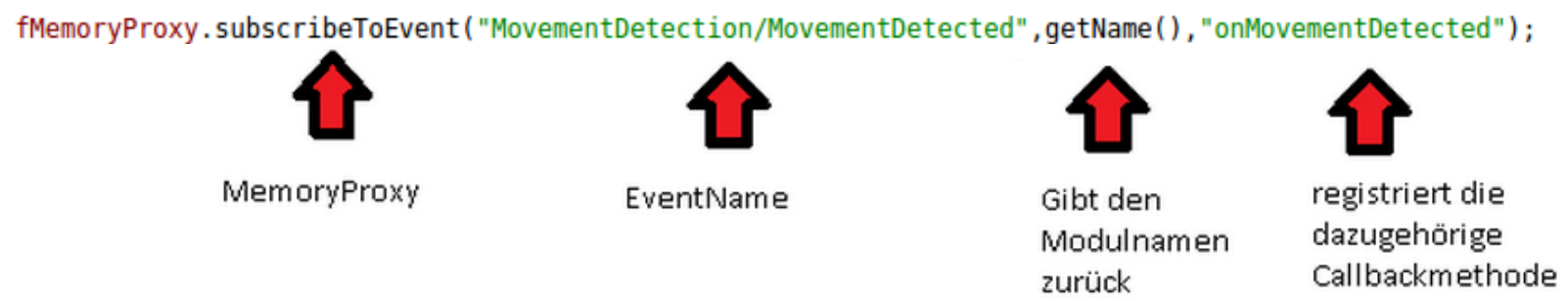

Abbildung 62 - Anmelden des Events MovementDetected am ALMemoryProxy 


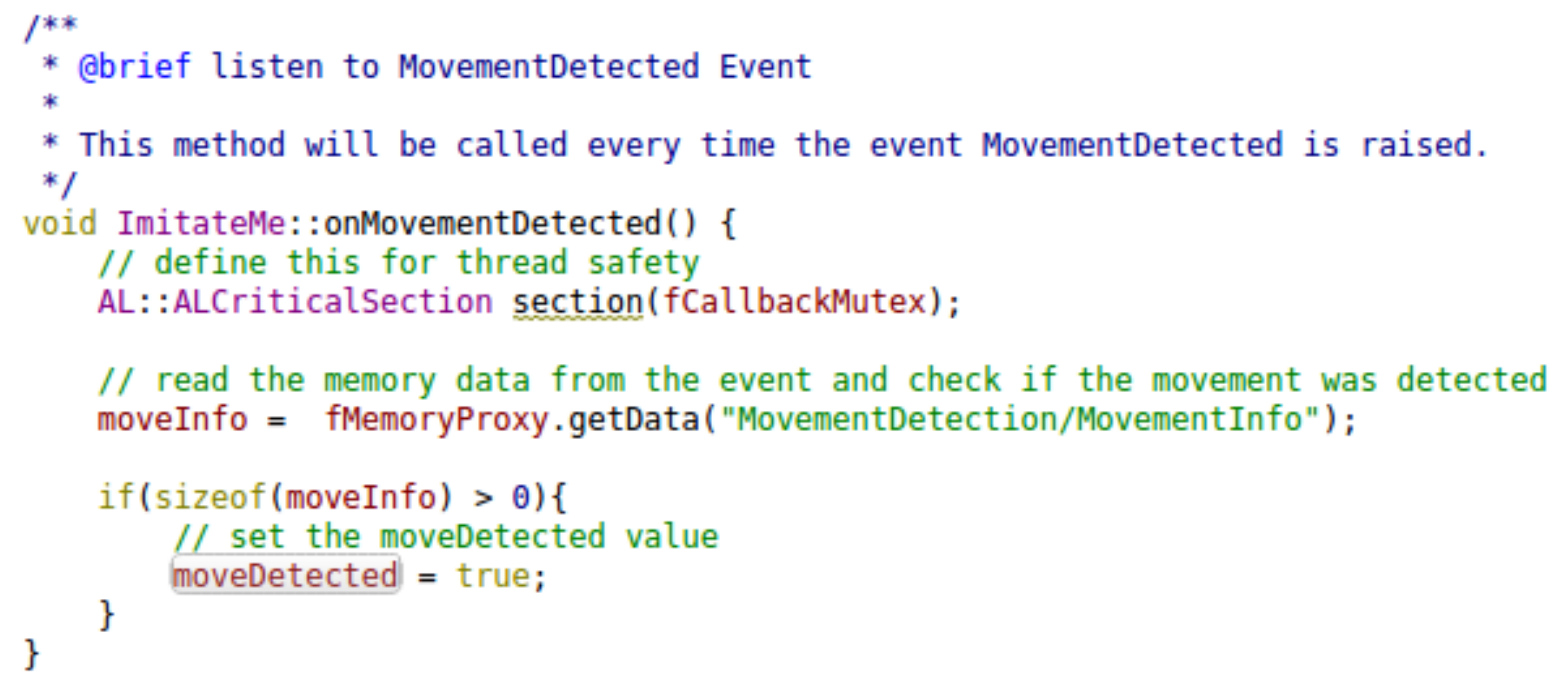

Abbildung 63 - Quellcodeausschnitt, imitate.cpp

In der dazugehörigen Callback-Methode onMovementDetected () im Codeabschnitt 63 wird mittels getData() der Speicherwert MovementDetection/MovementInfo ausgelesen und in die Variable moveInfo gespeichert. In diesem Fall wird geprüft, ob der ausgelesene Wert größter 0 ist und dann die Variable moveDetected auf true gesetzt.

Bei der Umsetzung stellte sich heraus, dass das Event ebenfalls auslöst, wenn der Roboter sich bewegt. Durch die Bewegung der Kamera ändert sich auch das aufgenommene Bild. Somit kann der Roboter, während er eine Bewegung ausführt, nicht feststellen ob eine andere Person zeitgleich sein Bewegungsmuster nachmacht. In der entstanden Version geht der NAO davon aus, dass der Betreuer den Aufforderungen, die Bewegungen nachzumachen, nachgekommen ist. 


\subsection{Modul „Geschichte“}

Das Modul „Geschichte“ wurde, wie im Teilkonzept 1 (Kapitel 5.1.3.) beschrieben, vollständig umgesetzt. Im Folgenden wird auf einige Besonderheiten bei der Umsetzung eingegangen. Der Quellcode und eine ausführliche Doxygen-Dokumentation befinden sich auf der DVD-Beigabe. Abbildung 64 zeigt die Projektstruktur:

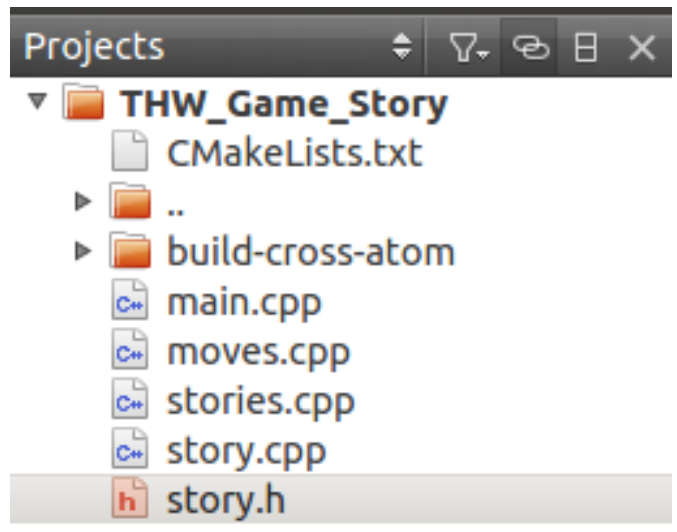

Abbildung 64 - Projektstruktur des Moduls „Geschichte“

Die story.cpp enthält die Hauptprogrammlogik. Die moves.cpp enthält die Bewegungen, die der NAO ausführen soll. In der stories. cpp sind die fünf Geschichten als TextToSpeech-Anweisungen in jeweils einer eigenen Methode eingebettet. Abbildung 65 zeigt beispielhaft die Implementierung anhand der Methode für das Gefühlmotiv „wütend“. Der Quelltextausschnitt zeigt, dass einfache Sätze verwendet wurden und jeder Satz in einer eigenen ttsProxy.say () -Anweisung aufgerufen wird. Das hat den Vorteil, dass zwischen den einzelnen Sprachausgaben eine kleine Pause entsteht, die das Zuhören erleichtert.

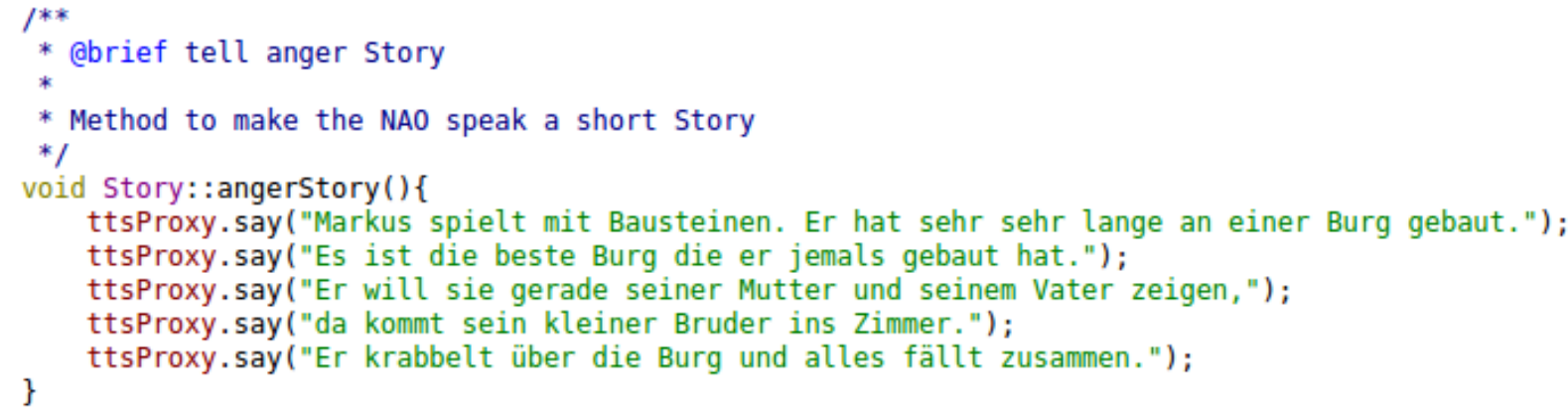

Abbildung 65 - Quellcodeausschnitt, angerStory(), stories.cpp

Für die Umsetzung war es vorgesehen, unter anderem den tag \rspd=75\zu verwenden, um die relative Sprechgeschwindigkeit auf $75 \%$ zu reduzieren (Abbildung 66). Das Einbinden jeglicher tags in den Sprachstring im C++-Quelltext zeigte auf dem Roboter jedoch 
keine Auswirkung. Ein Grund konnte, trotz sorgfältiger Fehleranalyse, bis zum Abschluss der Arbeit nicht gefunden werden. Mit einzelnen Sprachanweisungen pro Satz und zusätzlicher Kommasetzung wurden ersatzweise künstliche Pausen erzeugt, die das Textverständnis beim Zuhören erhöhen.

ttsProxy.say(" \rspd=75\Es ist die beste Burg, die er jemals gebaut hat.");

Abbildung 66 - Quellcodeausschnitt, tag in Sprachstring ohne Wirkung, stories . cpp

Folgende „Social Stories“ wurden vom Autor anhand von Anregungen aus der Literatur [52, 15, 53] entworfen, erarbeitet und im Modul integriert.

\begin{tabular}{|c|c|}
\hline Gefühlsmotiv & Text \\
\hline wütend & $\begin{array}{l}\text { Markus spielt mit Bausteinen. Er hat sehr, sehr lange an einer Burg gebaut. Es ist } \\
\text { die beste Burg, die er jemals gebaut hat. Er will sie gerade seiner Mutter und } \\
\text { seinem Vater zeigen, da kommt sein kleiner Bruder ins Zimmer. Er krabbelt über } \\
\text { die Burg und alles fällt zusammen. }\end{array}$ \\
\hline traurig & $\begin{array}{l}\text { Heute ist Olivias Geburtstag. Sie hat sich das ganze Jahr auf diesen Tag gefreut. } \\
\text { Sie hat sich einen großen Ball gewünscht. Jedem, den sie traf, erzählte sie von } \\
\text { diesem Wunsch. Doch an ihrem Geburtstag bekommt sie keinen Ball geschenkt. } \\
\text { Ihr Wunsch hatte sich nicht erfüllt. }\end{array}$ \\
\hline glücklich & $\begin{array}{l}\text { Andreas, seine Mutter, sein Vater und sein Bruder fahren mit dem Auto an den } \\
\text { Strand. Andreas kann es kaum erwarten aus dem Auto zu steigen. Er rennt zum } \\
\text { Meer und springt in die Wellen. Alle suchen Muscheln im Sand. Am Ende des } \\
\text { Tages bauen alle zusammen eine große Sandburg. Das ist ein wundervoller Tag. }\end{array}$ \\
\hline ängstlich & $\begin{array}{l}\text { Michael geht mit seiner Mutter spazieren. Plötzlich kommt ihnen ein großer } \\
\text { Hund entgegen. Er kommt genau auf Michael zu. Michael bekommt weiche Knie. } \\
\text { Er versteckt sich schnell hinter seiner Mutter. }\end{array}$ \\
\hline aufgeregt & $\begin{array}{l}\text { Am Morgen vor Peters erstem Schultag fühlt Peter beim Aufwachen ein starkes } \\
\text { Kribbeln in seinem Magen. Beim Frühstück hat er nicht einmal Appetit auf seine } \\
\text { Lieblingscornflakes. Je näher der erste Schultag kommt, desto weniger will Peter } \\
\text { hingehen. }\end{array}$ \\
\hline
\end{tabular}

Tabelle 17 - im Modul „Geschichte“ verwendete „Social Stories“

Um nach jeder Erzählung den Roboter nicht fünf Wörter zur Auswahl ausgeben zu lassen, wurde eine kleine Abfrage implementiert, die abhängig von der erzählten Geschichte nur drei oder zwei Antwortmöglichkeiten vorschlägt. Abbildung 67 zeigt den Ausschnitt, in dem zwischen den Geschichten „,wütend“, „,traurig“, ,glücklich“ und ,ängstlich“ oder „,aufgeregt“ entschieden wird.

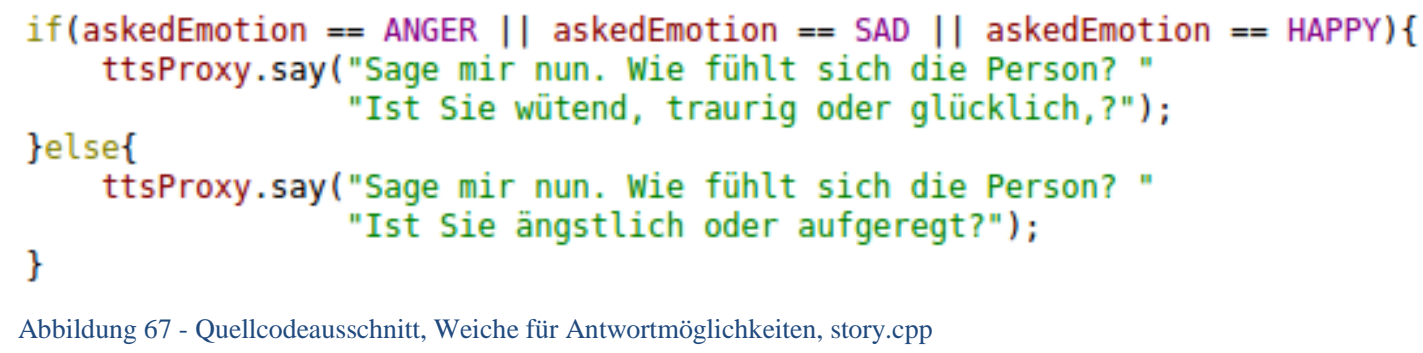

Abbildung 67 - Quellcodeausschnitt, Weiche für Antwortmöglichkeiten, story.cpp 


\section{Fazit}

Das in dieser Arbeit entwickelte Konzept stellt mit seinen vier entwickelten Modulen eine vielversprechende Grundlage für einen therapeutischen Einsatz des NAO-Roboters im Bereich der Autismus-Therapie dar. Abbildung 68 zeigt übersichtlich die entstandenen Module.

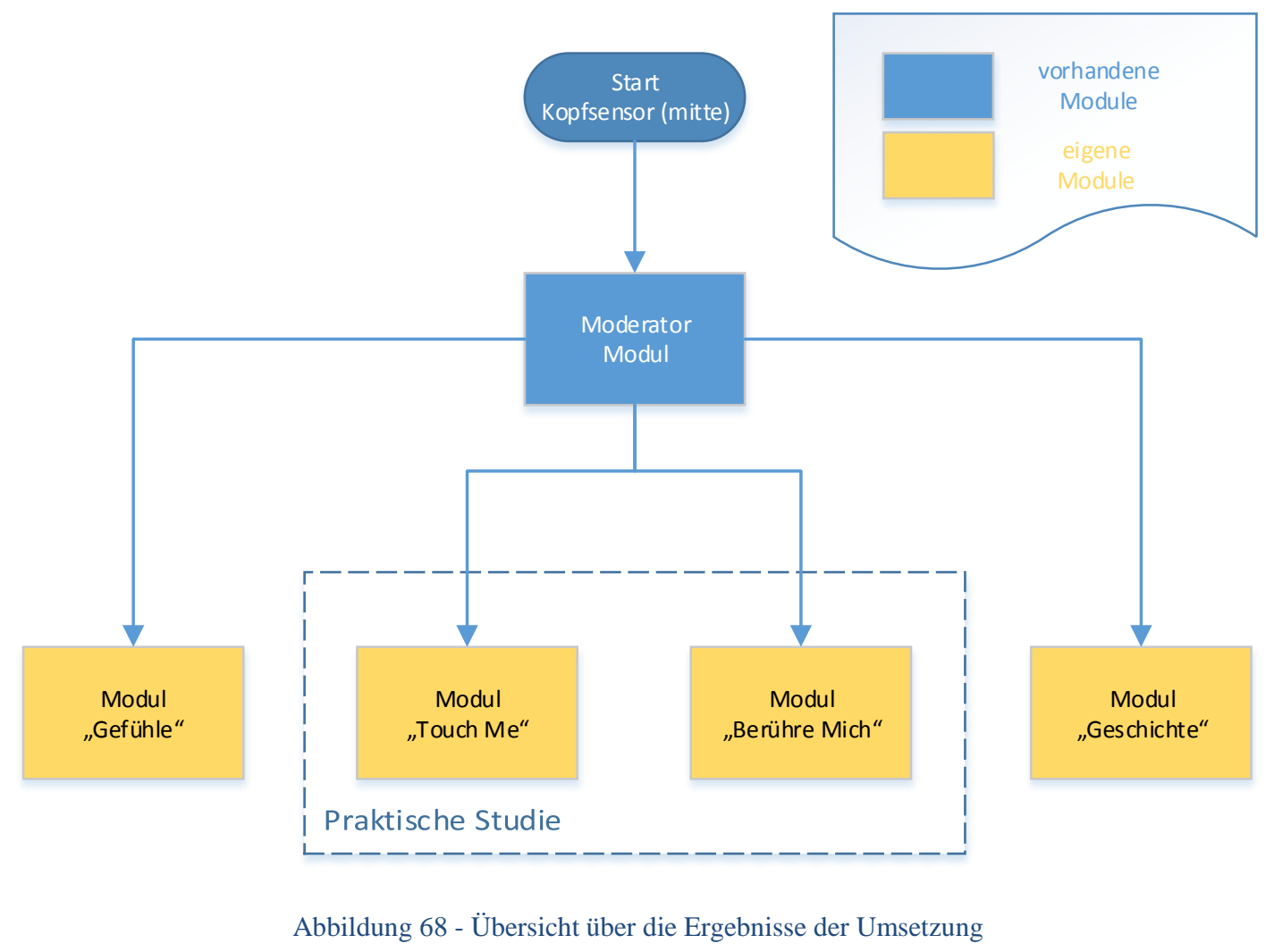

Mit dem Modul „Geschichte“ wurde ein essentieller Ansatz zur Förderung des Sozialen Verständnisses autistischer Kinder realisiert. Durch der Schilderung der Geschichten durch einen Roboter, der das Konzentrationsvermögen autistischer Kinder bewiesen erhöht, wird der Transfer des sozial-emotionalen Gehaltes der ,Social Storys“ noch verstärkt.

Das entwickelte Modul „Gefühle“ bietet dank des implementierten dreiteiligen Interaktionsmodells einen optimalen variablen sozialen Kontext, um analytisch Emotionen mit Gesten zu verknüpfen. Die dabei geförderte kognitive Empathie wird dem einen oder anderen autistischen Kind neue soziale Interaktionsmöglichkeiten mit anderen Menschen eröffnen. Die Beeinträchtigung durch defekte Spiegelneuronen kann somit im Ansatz kompensiert werden, da das Kind auf analytischem Wege die Emotionen lernt.

Die Ergebnisse der praktischen Studie mit Theo zeigen deutlich, wie effizient sich der NAO als „Social Assistive Robot“ einsetzen lässt. Er fungierte optimal als therapeutisches Mittel, 
um den in diesem Modul entwickelten Ansatz der Kommunikationsförderung zu realisieren. Durch die Erfahrung der Betreuer/innen im mehrjährigen Umgang mit Theo war ihre Wahl, Theo für dieses Projekt auszuwählen, ein entscheidender Schritt für die bisher erzielten Erfolge. Die Zusammenarbeit mit den Betreuer/innen des Therapiezentrums bei der Entwicklung der Module „Berühre Mich“ und „Touch Me“ zeigt, dass eine Brücke zwischen technologischem und therapeutischem Verständnis geschlagen werden kann.

Das Ergebnis der Evaluation der Entwicklerdokumentation hat gezeigt, dass es oft notwendig wird, mit mehreren Entwicklungswerkzeugen $\mathrm{zu}$ arbeiten, um optimale Programmierergebnisse zu erhalten.

Die Ausarbeitung zum Leap Motion Controller weist nur unter bestimmten Voraussetzungen auf eine optionale Integration in eine therapeutische Anwendung mit dem NAO hin. Vielmehr sieht der Autor hier ein Potential in der Realisierung von Anwendungen für gestengesteuerte Roboter, die über deutlich mehr Freiheitsgrade und Finger an einer Hand verfügen als der NAO-Roboter.

\section{Ausblick}

Um die Möglichkeiten des Moduls „Gefühle“ mit dem NAO zu steigern, empfiehlt sich die Verwendung einer seit 2001 oft in der HRI angewendeten Technik namens „Wizard of Oz“ [54]. Dabei wird der Roboter aus einem für das Kind nicht sichtbaren Bereich (Raum nebenan) von einer trainierten Person gesteuert. Die Person nebenan hat durch Bildschirme und Lautsprecher während der therapeutischen Sitzung eine ausreichende Übersicht über die Situation. Die Steuerung kann hierbei durch verschiedene Vorgehensweisen realisiert werden, wie sie Laurel Riek von der Universität Notre Dame beschreibt [54]. Zielführend empfiehlt sich für eine zukünftige Weiterentwicklung der Anwendung die Steuerung über eine Skeletterfassung (Skeleton Tracking) der Person, welche den NAO-Roboter im Raum nebenan steuert. Damit erweitert sich die Fähigkeit des Roboters, die Bewegungen des Kindes zu erkennen und direkt zu imitieren. Eine solche Skeletterkennung könnte durch den von Microsoft entwickelte Kinect Kamera Sensor* realisiert werden. Simon Päusch von der Hochschule Ulm untersuchte 2011 in seiner Bachelorarbeit mittels des Kinect Sensors eine Teleoperation mit dem NAO-Roboter [43]. Dieser Ansatz ließe sich nach Meinung des Autors in das derzeitige Modul „Gefühle“ integrieren, um dieses weiterzuentwickeln. 
Eine zusätzliche Weiterentwicklungsmöglichkeit sieht der Autor in der auf dem NAO vorhandenen Bildverarbeitungsbibliothek OpenCV*$V^{*}$. Hiermit ist die Möglichkeit einer intensiven Bilderkennung und deren Auswertung gegeben. Mit dieser Funktionalität wäre eine Gestenerkennung oder eine programmtechnische Auswertung des Blickkontakts denkbar.

Für die Weiterentwicklung des Moduls „Geschichte“ könnte es sinnvoll sein, zum einen, eine funktionierende Möglichkeit zu finden, den NAO langsamer sprechen zu lassen. Zum anderen wäre ab einer Repertoiregröße von mehr als sechs ,Social Stories“ die Implementierung einer lokalen Datenbank auf dem NAO vorteilhaft, um die Organisation der Geschichten zu vereinfachen.

Um die Entwicklungsreife der Module „Gefühle“ und „Geschichte“ zu testen, empfiehlt es sich diese in betreuten Sitzungen zusammen mit ausgewählten autistischen Kindern anzuwenden und gegebenenfalls anzupassen.

Die positive Entwicklung der Testreihe und die Ergebnisse des 4. Tests mit Theo weisen darauf hin, dass das entwickelte Modul in Zukunft weiterhin in therapeutischen Sitzungen Anwendung finden sollte, um die entstandene Bindung zwischen Theo und Lino auszubauen sowie weitere qualitative Lernerfolge zu erzielen

Mit den Ergebnissen dieser Arbeit könnte im Raum Berlin ein weiterer Entwicklungsstandort etabliert werden, um einen internationalen Austausch im Bereich der Human-RobotInteraction zu fördern. Es wurde speziell der Weg für einen Dialog zum Austausch von Therapieansätzen mit dem humanoiden Roboter NAO im autistischen Spektrum geebnet. 


\section{Glossar}

\begin{tabular}{|c|c|}
\hline Begriff & Erklärung \\
\hline Empathie & $\begin{array}{l}\text { Bereitschaft und Fähigkeit, sich in die Situation anderer } \\
\text { Menschen einzufühlen }\end{array}$ \\
\hline $\begin{array}{l}\text { Robot-based } \\
\text { Intervention Program }\end{array}$ & $\begin{array}{l}\text { Ein von der malaysischen Universität MARA entwickeltes } \\
\text { Testverfahren für Evaluationen mit dem Roboter NAO und } \\
\text { autistischen Kindern. }\end{array}$ \\
\hline $\begin{array}{l}\text { gestüitzte } \\
\text { Kommunikation }\end{array}$ & $\begin{array}{l}\text { Kommunikationshilfe, die die Lautsprache (verbale Sprache) } \\
\text { durch andere Kommunikationssysteme ersetzt. Beispiele sind } \\
\text { unteranderem Symbol-, oder Gebärdensysteme. }\end{array}$ \\
\hline Infrarot & $\begin{array}{l}\text { Für das menschliche Auge nicht sichtbare Lichtwellen im } \\
\text { Frequenzspektrum von } 0,78 \mu \mathrm{m} \text { bis } 1 \mathrm{~mm} \text {, nutzbar für } \\
\text { Datenübertragung }\end{array}$ \\
\hline Kinect Kamera Sensor & $\begin{array}{l}\text { Ein von Microsoft entwickelter Tiefensensor, der mittels } \\
\text { Infrarot das Auslesen von 3D-Tiefeninformationen einer von } \\
\text { der Kamera getätigten Aufnahme ermöglicht. }\end{array}$ \\
\hline Ethernet Port & $\begin{array}{l}\text { Netzwerkschnittstelle für einen kabelgebunden LAN-Anschluss } \\
\text { mit der IEEE-Spezifikation } 802.3\end{array}$ \\
\hline WLAN-Hotspot & $\begin{array}{l}\text { Lokaler Bereich, in dem ein Provider kabellos Internetzugang } \\
\text { zur Verfügung stellt. }\end{array}$ \\
\hline Tethering & $\begin{array}{l}\text { Verbindung eines Smartphones mit einem PC via WLAN oder } \\
\text { USB, um dem PC eine Internetverbindung über Mobilfunk zu } \\
\text { ermöglichen. }\end{array}$ \\
\hline Framework & $\begin{array}{l}\text { Rahmenstruktur mit Vorgabe der Anwendungsarchitektur für } \\
\text { die Entwicklung von Software in eine speziellen Sprache }\end{array}$ \\
\hline GNU-Lizenz & $\begin{array}{l}\text { freie Dokumentationslizenz, erlaubt die Vervielfältigung, } \\
\text { Verarbeitung und Veränderung des Werkes }\end{array}$ \\
\hline API & $\begin{array}{l}\text { Programmierschnittstelle, bietet Methoden und Klassen zum } \\
\text { Zugriff auf die darunter liegenden Schichten an. }\end{array}$ \\
\hline JNAOqi & $\begin{array}{l}\text { Programmbibliothek für die Softwareentwicklung für den NAO } \\
\text { mit Java }\end{array}$ \\
\hline $\begin{array}{l}\text { Kartesischen } \\
\text { Koordinatensystem* }\end{array}$ & $\begin{array}{l}\text { Koordinatensystem mit einer vom Ursprung ausgehenden } \mathrm{x}-, \mathrm{y}- \\
\text { und z-Achse, der Ursprung hat den Wert } 0\end{array}$ \\
\hline Audio Video Interleave & $\begin{array}{l}\text { Vom Microsoft definiertes Videoformat, in dem die Audio- und } \\
\text { die Videodatei ineinander verzahnt abgespeichert werden, um } \\
\text { Speicherplatz zu gewinnen und eine höhere Datenrate zu } \\
\text { erreichen }\end{array}$ \\
\hline Keyframe & $\begin{array}{l}\text { Einzelne feste Bewegungsposition, mehrere Keyframes ergeben } \\
\text { einen Bewegungsablauf }\end{array}$ \\
\hline $\begin{array}{l}\text { Waveform Audio File } \\
\text { Format }\end{array}$ & $\begin{array}{l}\text { Containerformat für eine unkomprimierte digitale Speicherung } \\
\text { von Audiodaten }\end{array}$ \\
\hline
\end{tabular}




\begin{tabular}{|l|l|}
\hline Begriff & Erklärung \\
\hline MPEG Audio Layer III & $\begin{array}{l}\text { Verfahren zur verlustbehafteten Kompression von digitalen } \\
\text { Audiodaten, Kompressionsfaktor }>10\end{array}$ \\
\hline $\begin{array}{l}\text { Doxygen- } \\
\text { Dokumentation }\end{array}$ & $\begin{array}{l}\text { Doxygen ist ein Standard-Tool, um eine übersichtliche HTML- } \\
\text { Dokumentation ursprünglich aus C++-Quellcode, mittlerweile } \\
\text { aber auch aus anderen Programmiersprachen zu generieren. }\end{array}$ \\
\hline RGB-Farbraum & $\begin{array}{l}\text { Ein additiver Farbraum der durch das Mischen der Grundfarben } \\
\text { Rot, Grün und Blau die Farbwahrnehmung nachbildet }\end{array}$ \\
\hline
\end{tabular}




\section{Abkürzungsverzeichnis}

\begin{tabular}{|l|l|}
\hline Abkürzung & Bedeutung \\
\hline IEEE & Institute of Electrical and Electronics Engineers \\
\hline ACM & Association for Computing Machinery \\
\hline RBIP & Robot-based Intervention Program \\
\hline API & Application Programming Interface \\
\hline JVM & Java Virtual Machine \\
\hline IDE & Integrated Development Environment \\
\hline LAN & Local Area Network \\
\hline WLAN & Wireless (kabellos) Local Area Network \\
\hline AVI & Audio Video Interleave \\
\hline WAVE & Waveform Audio File Format \\
\hline MP3 & MPEG Audio Layer III \\
\hline RGB & Rot Grün Blau \\
\hline
\end{tabular}




\section{Abbildungsverzeichnis}

Abbildung 1 - Der humanoide NAO-Roboter Quelle: Aldebaran Robotics .......................... 1

Abbildung 2 - Triad of Impairments ................................................................................ 7

Abbildung 3 - soziales Signal Quelle: [14], editiert vom Autor ........................................... 9

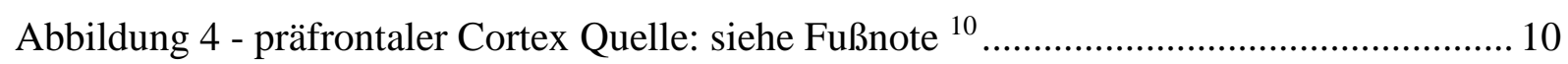

Abbildung 5 - The FACE Android Quelle: siehe Fußnote ${ }^{16}$............................................... 13

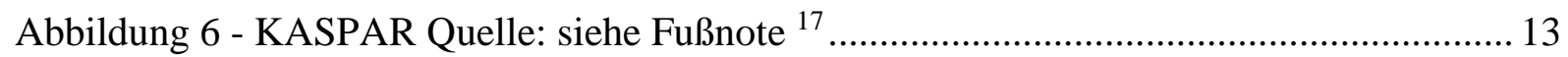

Abbildung 7 - Bewegungsspielraum des NAO-Roboters ................................................ 15

Abbildung 8 - Zeit des Blickkontakts im Vergleich RBIP und Klassenraum Quelle: [17] ..... 16

Abbildung 9 - Stereotyp Verhalten RBIP gegenüber Klassenraum Quelle: [18], editiert vom

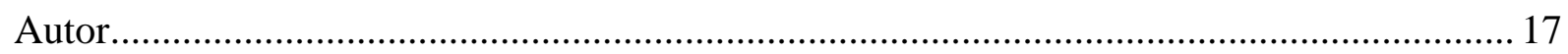

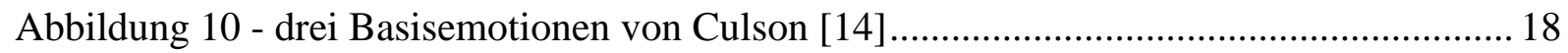

Abbildung 11 - fünf ausgewählte Körperhaltungen für ,wütend“ Quelle: [14]...................... 19

Abbildung 12 - fünf ausgewählte Körperhaltungen für ,glücklich“ Quelle: [14] .................. 19

Abbildung 13 - fünf ausgewählte Körperhaltungen für "traurig" Quelle: [14] ....................... 19

Abbildung 14 - zweiteiliges Interaktionsmodell Quelle: [22] ............................................. 20

Abbildung 15 - dreiteiliges Interaktionsmodell Quelle: [22] .............................................. 21

Abbildung 16 - taktile und mechanische Sensoren Quelle: Aldebaran Robotics..................... 23

Abbildung 17 - CHEST-Button - Knopf auf der Brust Quelle: Aldebaran Robotics ............. 23

Abbildung 18 - mechanischer Schalter am Fuß Quelle: Aldebaran Robotics.......................... 23

Abbildung 19 - drei taktile Kopfsensoren Quelle: Aldebaran Robotics, editiert vom Autor... 24

Abbildung 20 - taktile Handsensoren Quelle: Aldebaran Robotics, editiert vom Autor ......... 24

Abbildung 21 - LEDs an den Augen Quelle: Aldebaran Robotics ....................................... 25

Abbildung 22 - Mikrofone des NAO-Roboters Quelle: Aldebaran Robotics ......................... 25

Abbildung 23 - Lautsprecher des NAO-Roboters Quelle: Aldebaran Robotics ..................... 26

Abbildung 24 - Kameras am NAO Quelle: Aldebaran Robotics .......................................... 26

Abbildung 25 - Gelenke und Freiheitsgrade des NAO Quelle: Aldebaran Robotics............... 27

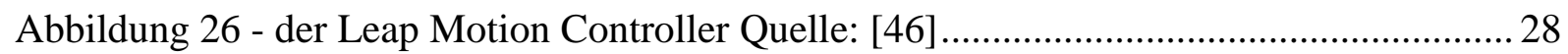

Abbildung 27 - Interaktionsraum über dem Controller, Quelle [46] .................................... 28

Abbildung 28 - Kartesisches Koordinatensystem des Leap-Motion Controllers Quelle: [23] 29

Abbildung 29 - Trackingdaten die vom Controller geliefert werden Quelle: [23] .................. 30

Abbildung 30 - CircleGesture Quelle [24] ..................................................................... 30

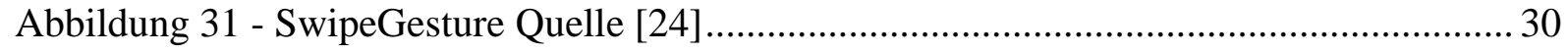

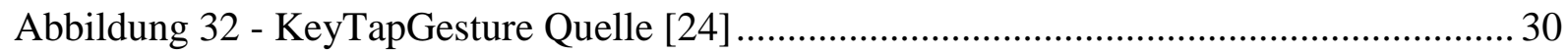

Benjamin Körner $\quad$ XIV 


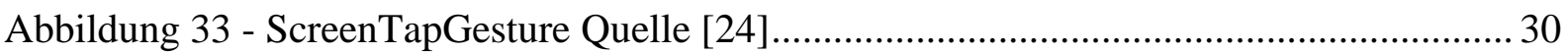

Abbildung 34 - Zugriff und Erfassung der Fingerspitzen Quelle: [23]................................. 31

Abbildung 35 - Architektur des NAOqi-Frameworks Quelle: Aldebaran Robotics ................ 33

Abbildung 36 - Zusammenhang: Broker, Module und Methoden Quelle: Aldebaran Robotics 34

Abbildung 37 - Übersicht der Programmiertools Quelle: Aldebaran Robotics ....................... 35

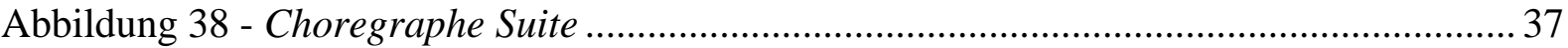

Abbildung 39 - Java Syntax für einen Modulaufruf Quelle: Aldebaran Robotics .................. 40

Abbildung 40 - Zuordnung der Module zu Hauptbeeinträchtigungen im autistischen Spektrum .44

Abbildung 41 - Interaktionsradius des NAO Quelle: Aldebaran Robotics ............................. 45

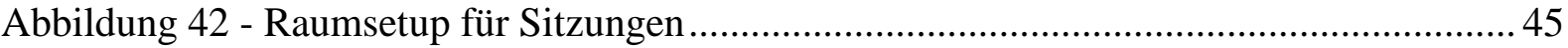

Abbildung 43 - dreiteiliges Interaktionsmodell Quelle: [22] .............................................. 53

Abbildung 44 - Extraktion der Emotionen "wütend", "glücklich" und "traurig" Quelle: [22] 54

Abbildung 45 - Programlogik des Moduls "Gefühle" ............................................................. 57

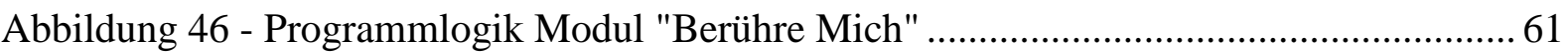

Abbildung 47 - Programmablaufplan Modul 3 "Geschichte" ................................................ 65

Abbildung 48 - Aufbau einer Leap Motion-Sitzung mittels eines Notebooks......................... 68

Abbildung 49- Programmablaufplan für Modul mit Leap Motion Controller......................... 69

Abbildung 50- Testraum im Frühförderzentrum in Berlin Quelle: eigenes Foto ................... 70

Abbildung 51 - Behaviors für den Erstkontakt, erstellt mit Choregraphe ............................. 72

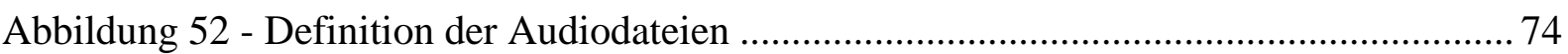

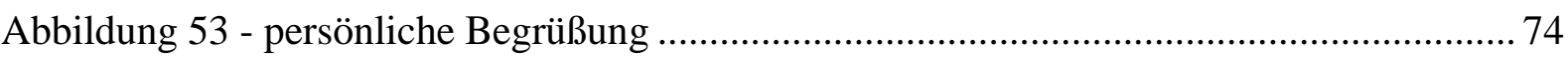

Abbildung 54 - Eingefügte Bewegungen hinter verbalen Aufforderungen, touchme.cpp

Abbildung 55 - Bewegungsänderung der Fuß- und Armgelenke …...................................... 76

Abbildung 56 - Programmablaufplan Modul 2 „Berühre Mich“, angepasste Version ............ 79

Abbildung 57 - Projektstruktur des Moduls „Gefühle“ ............................................................ 80

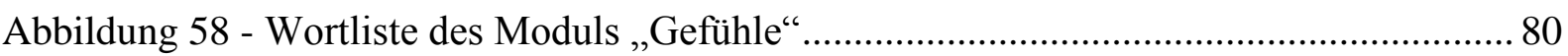

Abbildung 59 - Quellcodeausschnitt, imitateMe.cpp........................................................ 81

Abbildung 60 - Quellcodeausschnitt, moves.cpp Methode: motion:sad() ............................. 81

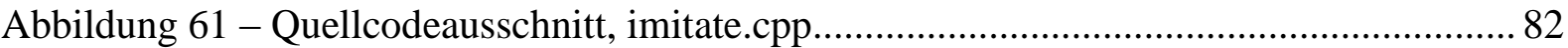

Abbildung 62 - Anmelden des Events MovementDetected am ALMemoryProxy........82

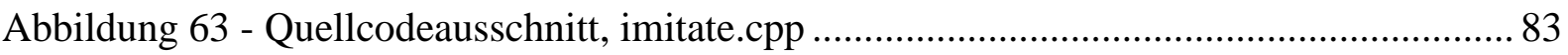

Benjamin Körner $\quad$ XV 
Abbildung 64 - Projektstruktur des Moduls „Geschichte“........................................................ 84

Abbildung 65 - Quellcodeausschnitt, angerStory(), stories. cpp ..................................... 84

Abbildung 66 - Quellcodeausschnitt, tag in Sprachstring ohne Wirkung, stories . cpp ... 85

Abbildung 67 - Quellcodeausschnitt, Weiche für Antwortmöglichkeiten, story.cpp .............. 85

Abbildung 68 - Übersicht über die Ergebnisse der Umsetzung ........................................... 86

Abbildung 69 - CMake Wizard ..................................................................................XVII

Abbildung 70 - Auswahl des build-cross-atom Ordners......................................XXVII

Abbildung 71 - CMake Wizard - Run CMake um das Projekt zu initialisieren ............. XXVIII

Abbildung 72 - Methodenübersicht und Aufrufabhängigkeiten des Moderator Moduls ... XXIX

Abbildung 73 - Methodenübersicht und Aufrufabhängigkeiten des Moduls „Gefühle“ ... XXIX

Abbildung 74 - Methodenübersicht und Aufrufabhängigkeiten der Module „Berühre Mich“ und „Touch $\mathrm{Me}^{\text {“ }}$

XXX

Abbildung 75 - Methodenübersicht und Aufrufabhängigkeiten des Moduls „Geschichte“ XXX 


\section{Tabellenverzeichnis}

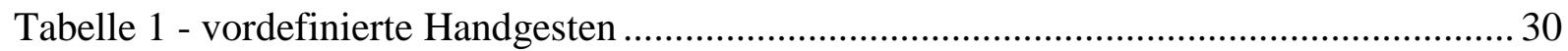

Tabelle 2 - Modulübersicht im NAOqi-Framework ............................................................ 33

Tabelle 3 - Bewertungskriterien der Frameworks Quelle: eigene Tabelle.............................. 36

Tabelle 4 - NAOqi-Events Quelle: Aldebaran Robotics, editiert vom Autor .......................... 39

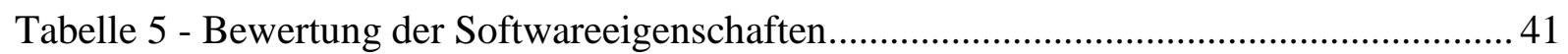

Tabelle 6 - Voraussetzungen für den Erfolg der Anwendung ............................................... 43

Tabelle 7 - Wertungsmatrix für Erstkontakt mit dem NAO Quelle: eigene Tabelle ............... 48

Tabelle 8 - Hardwareanforderungen, die für alle Module gelten ........................................... 50

Tabelle 9 - Farbcodezuordnung für die einzelnen Module ................................................. 51

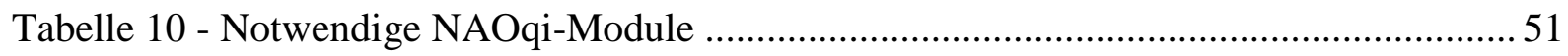

Tabelle 11 - Zuordnung der NAOqi-Events zu den einzelnen Modulen ................................ 51

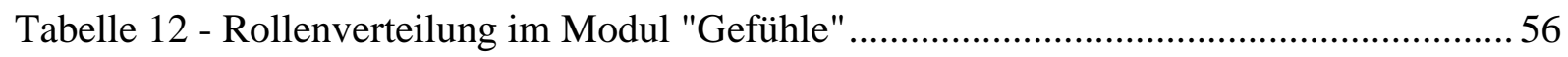

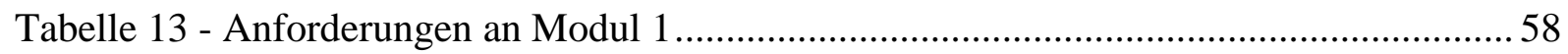

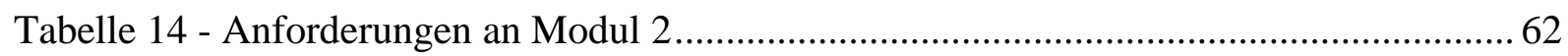

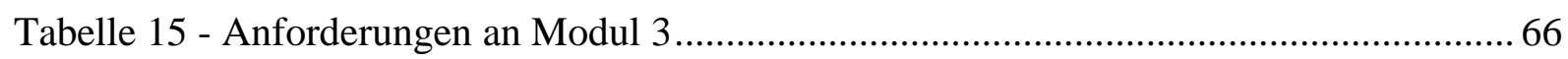

Tabelle 16 - Auswahl an tags, die die Sprachausgabe manipulieren Quelle: [49].................. 67

Tabelle 17 - im Modul „Geschichte“ verwendete „Social Stories“ "........................................ 85 


\section{Literaturverzeichnis}

[1] Bredenfeld, A. Dr. 2014. German Open 2014. http://www.robocupgermanopen.de/de/robocup. Zugriff: 8 Mai 2014.

[2] ALIZ-E Projekt. 2014. ALIZ-E research. http://fostsvn.uopnet.plymouth.ac.uk/index.php?option=com_content\&view=article\&id= 46\&Itemid=53. Zugriff: 2 April 2014.

[3] Gupta, S. K. 2014. When Will We Have Robots To Help With Household Chores? http://spectrum.ieee.org/automaton/robotics/home-robots/when-will-we-have-robots-tohelp-with-household-chores. Zugriff: 19 Juni 2014.

[4] Shamsuddin, S., Yussof, H., Ismail, L. I., Mohamed, S., Hanapiah, F. A., and Zahari, N. I. 2012. Initial Response in HRI- a Case Study on Evaluation of Child with Autism Spectrum Disorders Interacting with a Humanoid Robot NAO. Procedia Engineering 41, $1448-1455$.

[5] Tanaka, F. and Matsuzoe, S. 2014. Children Teach a Care-Receiving Robot to Promote Their Learning: Field Experiments in a Classroom for Vocabulary Learning. http://humanrobotinteraction.org/journal/index.php/HRI/article/view/12/33. Zugriff: 18 Juni 2014.

[6] Tapus, A., Peca, A., Aly, A., Pop, C., Jisa, L., Pintea, S., Rusu, A. S., and David, D. O. 2012. Children with autism social engagement in interaction with Nao, an imitative robot: A series of single case experiments. IS 13, 3, 315-347.

[7] 2010 IEEE International Conference on Robotics and Automation (ICRA 2010).

[8] Baird, G., Simonoff, E., Pickles, A., Chandler, S., Loucas, T., Meldrum, D., and Charman, T. 2006. Prevalence of disorders of the autism spectrum in a population cohort of children in South Thames: the Special Needs and Autism Project (SNAP). The Lancet $368,9531,210-215$.

[9] Yussof, H. and Shamsuddin, S. Human-Robot Interaction Intervention Therapy Procedure for Initial Response of Autism Children with Humanoid Robot. Joint International Symposium on System-Integrated Intelligence 2012.

[10] Bhat, A. 2008. Autismus-Therapie: Roboter nimmt Angst vor Menschen - SPIEGEL ONLINE. http://www.spiegel.de/wissenschaft/mensch/autismus-therapie-roboter-nimmtangst-vor-menschen-a-569595.html. Zugriff: 2 April 2014.

[11] Michlo, K. 2011. Möglichkeiten strukturierter und visualisierter Förderung für SchülerInnen und Schüler mit Autismus, Berlin. 
[12] Remschmidt, H. 2008. Autismus. Erscheinungsformen, Ursachen, Hilfen. Beck'sche Reihe 2147 : C.H. Beck Wissen. Beck, München.

[13] Resch, F. 1999. Entwicklungspsychopathologie des Kindes- und Jugendalters. Ein Lehrbuch. Anwendung Psychologie. Beltz, Weinheim.

[14] Chaby, L., Chetouani, M., Plaza, M., and Cohen, D. 2012. Exploring Multimodal SocialEmotional Behaviors in Autism Spectrum Disorders: An Interface between Social Signal Processing and Psychopathology. In Privacy, Security, Risk and Trust (PASSAT), 2012 International Conference on and 2012 International Confernece on Social Computing (SocialCom), 950-954.

[15] Grey, C. 2014. Social Stories ${ }^{\mathrm{TM}}$. http://www.thegraycenter.org/social-stories. Zugriff: 31 Juli 2014.

[16] Oberman, L. M., Hubbard, E. M., McCleery, J. P., Altschuler, E. L., Ramachandran, V. S., and Pineda, J. A. 2005. EEG evidence for mirror neuron dysfunction in autism spectrum disorders. Cognitive Brain Research 24, 2, 190-198.

[17] DÜLLINGS, C. 2013. Spiegelneuronen - Gehirnzellen, die emotionale Empathie ermöglichen. http://www.empathie-lernen.de/spiegelneuronen-emotionale-empathie. Zugriff: 4 Juni 2014

[18] Spektrum. Bei Autisten ist die Funktion der Spiegelneuronen gestört. http://www.spektrum.de/alias/psychische-krankheiten/bei-autisten-ist-die-funktion-derspiegelneuronen-gestoert/776478. Zugriff: 14 April 2014.

[19] Wada, K., Shibata, T., Saito, T., and Tanie, K. 2002. Robot assisted activity for elderly people and nurses at a day service center. In Robotics and Automation, 2002. Proceedings. ICRA '02. IEEE International Conference on, 1416-1421.

[20] 2007. Socially assistive robotics for post-stroke rehabilitation. BioMed Central.

[21] Mataric, M. J. 2007. Socially assistive robotics for post-stroke rehabilitation. BioMed Central Ltd (Feb. 2007).

[22] Diehl, J. J., Crowell, C. R., Villano, M., Wier, K., Tang, K., and Riek, L. D. 2014. Clinical Applications of Robots in Autism Spectrum Disorder Diagnosis and Treatment. In Comprehensive Guide to Autism. Clinical, V. B. Patel, V. R. Preedy and C. R. Martin, Eds. Springer New York, New York, NY, 411-422.

[23] Fridin, M. 2011. Educational Robot for Children with ADHD/ADD. http://asknao.aldebaran-

robotics.com/images/papers/fridinyaakobi_2011_educationalrobot.pdf. Zugriff: 1 April 2014. 
[24] Shamsuddin, S., Yussof, H., Ismail, L. I., Mohamed, S., Hanapiah, F. A., and Zahari, N. I. 2012. Humanoid Robot NAO Interacting with Autistic Children of Moderately Impaired Intelligence to Augment Communication Skills. Procedia Engineering 41, $1533-1538$.

[25] Thill, S., Pop, C. A., Belpaeme, T., Ziemke, T., and Vanderborght, B. 2012. Robotassisted therapy for autism spectrum disorders with (partially) autonomous control: Challenges and outlook. Paladyn 3, 4, 209-217.

[26] Feil-Seifer, D. and Mataric, M. 2011. Socially Assistive Robotics. IEEE Robot. Automat. Mag. 18, 1, 24-31.

[27] Ismail, L. I., Shamsudin, S., Yussof, H., Hanapiah, F. A., and Zahari, N. I. 2012. Estimation of Concentration by Eye Contact Measurement in Robot-based Intervention Program with Autistic Children. Procedia Engineering 41, 1548-1552.

[28] Bird, G., Leighton, J., Press, C., and Heyes, C. 2007. Intact automatic imitation of human and robot actions in autism spectrum disorders. Proc. Biol. Sci. 274, 1628, 3027-3031.

[29] Dautenhahn, K. Prof. 2013. The AuRuRA Project. http://www.aurora-project.com/. Zugriff: 26 Juni 2014.

[30] Mazzei, D., Billeci, L., Armato, A., Lazzeri, N., Cisternino, A., Pioggia, G., Igliozzi, R., Muratori, F., Ahluwalia, A., and Rossi, D. de. 2010. The FACE of autism. In RO-MAN, 2010 IEEE, 791-796.

[31] Scassellati, B., Henny Admoni, and Matarić, M. 2012. Robots for Use in Autism Research. Аnnu. Rev. Biomed. Eng. 14, 1, 275-294.

[32] Robins, B., Dautenhahn, K., and Dubowski, J. 2006. Does appearance matter in the interaction of children with autism with a humanoid robot? IS 7, 3, 509-542.

[33] Ricks, D. J. and Colton, M. B. Trends and considerations in robot-assisted autism therapy. In 2010 IEEE International Conference on Robotics and Automation (ICRA 2010), 4354-4359.

[34] Ismail, L. I., Shamsudin, S., Yussof, H., Hanapiah, F. A., and Zahari, N. I. 2012. Robotbased Intervention Program for Autistic Children with Humanoid Robot NAO: Initial Response in Stereotyped Behavior. Procedia Engineering 41, 1441-1447.

[35] Lakes Country Service Cooperative. Gilliam Autism Rating Scale (GARS-2). http://www.lcsc.org/cms/lib6/MN01001004/Centricity/Domain/15/EvaluationTemplateG illiamAutismRatingScale2.doc.

[36] Erden, M. S. Postural Expressions of Emotions in a Humanoid Robot for Assistive Applications. 
http://www.researchgate.net/publication/228443478_Postural_expressions_of_emotions_ in_a_humanoid_robot_for_assistive_applications/file/60b7d52550e0b631df.pdf. Zugriff: 30 Juli 2014.

[37] Erden, M. S. 2013. Emotional Postures for the Humanoid-Robot Nao. Int J of Soc Robotics 5, 4, 441-456.

[38] Coulson, M. 2004. Attributing emotion to static body postures: recognition accuracy, confusions, and viewpoint dependence. Springer Netherlands.

[39] Ekman, P. and Friesen, W. V. 1978. Facial action coding system. Consulting Psychologists Press, Palo Alto, Calif.

[40] Jungsik, H., Min, G. K., and Kwangsu, C. 2013. Developing interaction scenarios of robot-mediated imitation training for children with Autism Spectrum Disorders. In $R O$ MAN, 2013 IEEE, 616-620.

[41] Esubalew T. Bekele, UTTAMA LAHIRI, Julie Davidson, Zachary Warren, Nilanjan Sarkar. Development of a Novel Robot-Mediated Adaptive Response System for Joint Attention Task for Children with Autism.

[42] Shamsuddin, S. 2011. Humanoid Robot NAO: Review of Control and Motion Exploration.

[43] Simon Päusch. Teleoperation des humanoiden Roboters NAO per Kinect-Kamera. http://www.zafh-servicerobotik.de/ULM/dokumente/bachelor-simon-paeusch.pdf.

[44] Solid Works. 2014. NAO Roboter hilft Menschen mit Autismus. http://www.solidworks.de/sw/news/167_16563_DEU_HTML.htm. Zugriff: 24 März 2014.

[45] Bosse, T., Gerritsen, C., Man, J., and Treur, J. 2013. Effects of Virtual Training on Emotional Response. In Brain and Health Informatics, D. Hutchison, T. Kanade, J. Kittler, J. M. Kleinberg, F. Mattern, J. C. Mitchell, M. Naor, O. Nierstrasz, C. Pandu Rangan, B. Steffen, M. Sudan, D. Terzopoulos, D. Tygar, M. Y. Vardi, G. Weikum, K. Imamura, S. Usui, T. Shirao, T. Kasamatsu, L. Schwabe and N. Zhong, Eds. Lecture notes in computer science. Springer International Publishing, Cham, 21-32.

[46] Leap Motion Ltd. 2014. API Overview - Leap Motion C\# and Unity SDK v2.0 Beta documentation.

https://developer.leapmotion.com/documentation/skeletal/csharp/devguide/Leap_Overvie w.html. Zugriff: 8 Juli 2014. 
[47] Labbé, G. and Mohnke, J. 2013. Entwicklung von Beispielanwendungen für das Funktionsspektrum des NAO-Roboters und Vertiefung anhand einer komplexen Applikation. TH, Wildau.

[48] Oberman, L. M., Hubbard, E. M., McCleery, J. P., Altschuler, E. L., Ramachandran, V. S., and Pineda, J. A. 2005. EEG evidence for mirror neuron dysfunction in autism spectrum disorders. Brain Res Cogn Brain Res 24, 2, 190-198.

[49] Acapela Group. 2013. Text Tag Documentaion. https://community.aldebaran.com/doc/114/_downloads/audio_system_acapelamobilitytexttags.pdf. Zugriff: 30 Juli 2014.

[50] Autismus Deutschland, Landesverband Berlin e.V. 2014. Ganztags-Kleinklassen | Autismus Berlin. http://www.autismus-berlin.de/kleinklassen.php. Zugriff: 13 Juni 2014.

[51] Aldebaran Robotics. 2014. Overview - Aldebaran 2.1.1.11 documentation. https://community.aldebaran.com/doc/2-1/. Zugriff: 30 Juli 2014.

[52] Skinner, R. 2014. Free Social Stories - Printable Examples. http://www.child-behaviorguide.com/free-social-stories.html. Zugriff: 31 Juli 2014.

[53] Vihar, J. 1999. Autism Network, December 1999. http://www.autismindia.org/dec99.html. Zugriff: 1 August 2014.

[54] Riek, L. 2012. Wizard of Oz Studies in HRI: A Systematic Review and New Reporting Guidelines. JHRI, 119-136.

[55] Lipinski, K. 2014. IT-Lexikon: Fachwissen für IT-Professionals - ITWissen.info -. http://www.itwissen.info/. Zugriff: 30 Juli 2014. 


\section{A. Anhang}

\section{A.1. Erstellung von Motions mittels des Animationsmodus und Export in C++}

Wie Motions und Bewegungsmuster mit dem Animationsmodus erstellt und in $\mathrm{C}++$ Quellcode verwendet werden können, wird in folgendem Tutorial beschrieben. Alle Abbildungen in diesem Tutorial sind eigens vom Autor erstellt. Es wurde deshalb auf eine Quellenangabe verzichtet.

Öffnen Sie Choregraphe

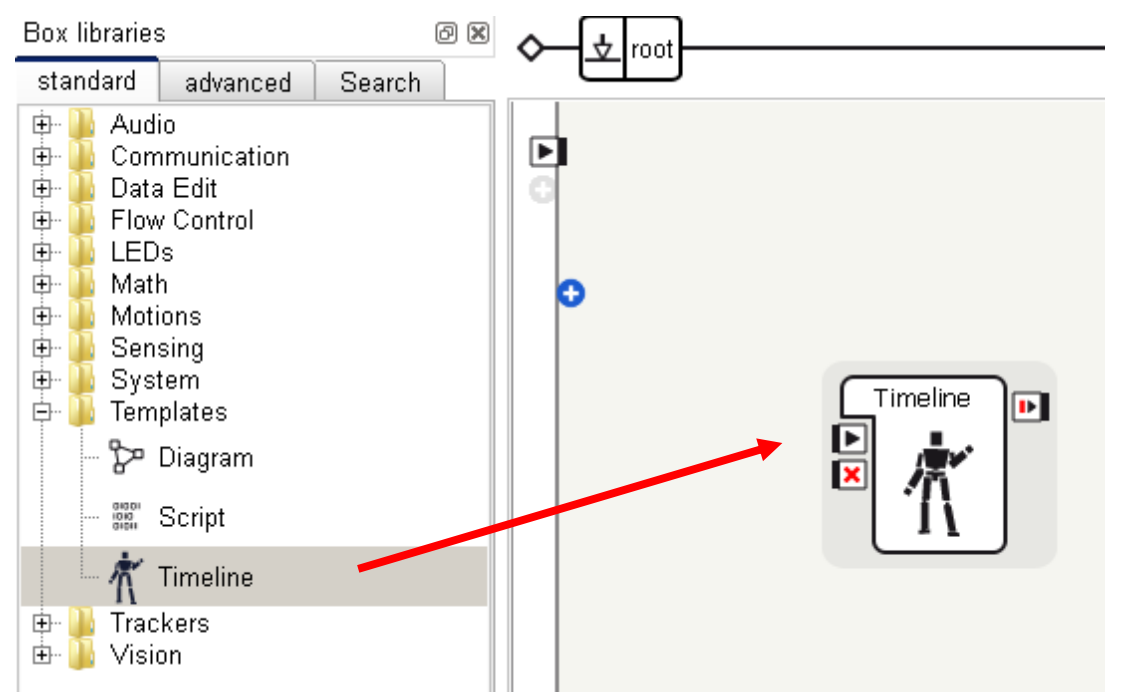

Ziehen Sie eine Timeline Box in das flow diagram. Doppelklicken Sie auf die Box. Klicken Sie auf das Stiftsymbol, um den erweiterten Timeline-Editor zu öffnen. Mit ihm lassen sich Bewegungen bequemer und effizienter entwickeln.

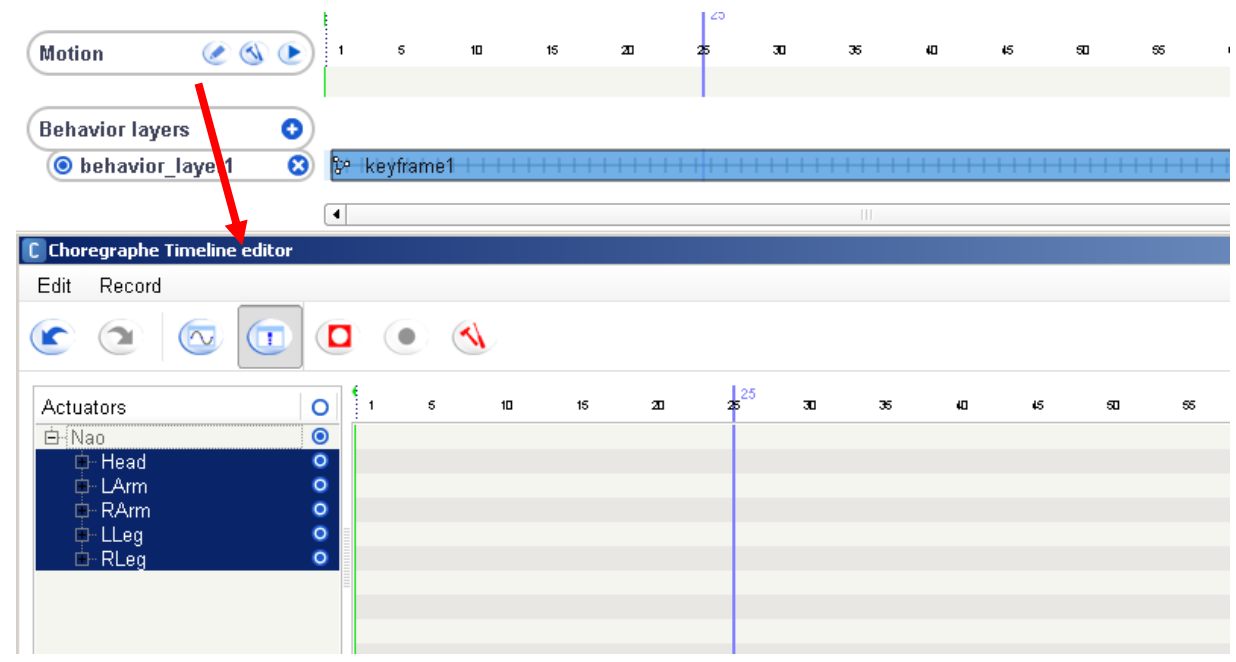

Im Timeline-Editor ist es möglich, jede Position einzelner Gelenke und Körperteile zu speichern und zu konfigurieren. Der Animationsmodus ist die einfachste Art und Weise 
Bewegungsabläufe aufzuzeichnen. In diesem Modus ist es möglich, den NAO wie eine Puppe in eine beliebige Position zu bewegen und die aktuellen Winkelwerte in einem Keyframe zu speichern. Verbinden Sie dazu ihrem Roboter mit Choregraphe. Klicken Sie auf das Symbol, um alle Motoren zu aktivieren und die stiffness einzuschalten. Klicken Sie danach auf das Symbol $\times$, um den Animationsmodus zu starten. Nun befindet sich der Roboter im Wartezustand. Um nun einen Arm oder ein Bein bewegen zu können, müssen Sie für jedes Körperteil, welches Sie bewegen wollen, die stiffness kurz abschalten. Folgende Übersicht zeigt die Sensoren, die dafür jeweils berührt werden müssen.

\begin{tabular}{|l|l|}
\hline Teil & Taktiler Sensor \\
\hline Kopf & Berühre den mittleren Kopfsensor \\
\hline Arme & $\begin{array}{l}\text { Halte die die Hand des NAO-Roboters umschlossen, } \\
\text { um durch die Berührung der Handsensoren den Arm } \\
\text { freizugeben }\end{array}$ \\
\hline Beine & Drücke den jeweiligen Fußsensor \\
\hline
\end{tabular}

Nachdem Sie z.B. den Arm gehoben haben, drücken Sie F8, um die Position in einem Keyframe zu speichern. Im Timeline-Editor sind die Keyframes aufgelistet.

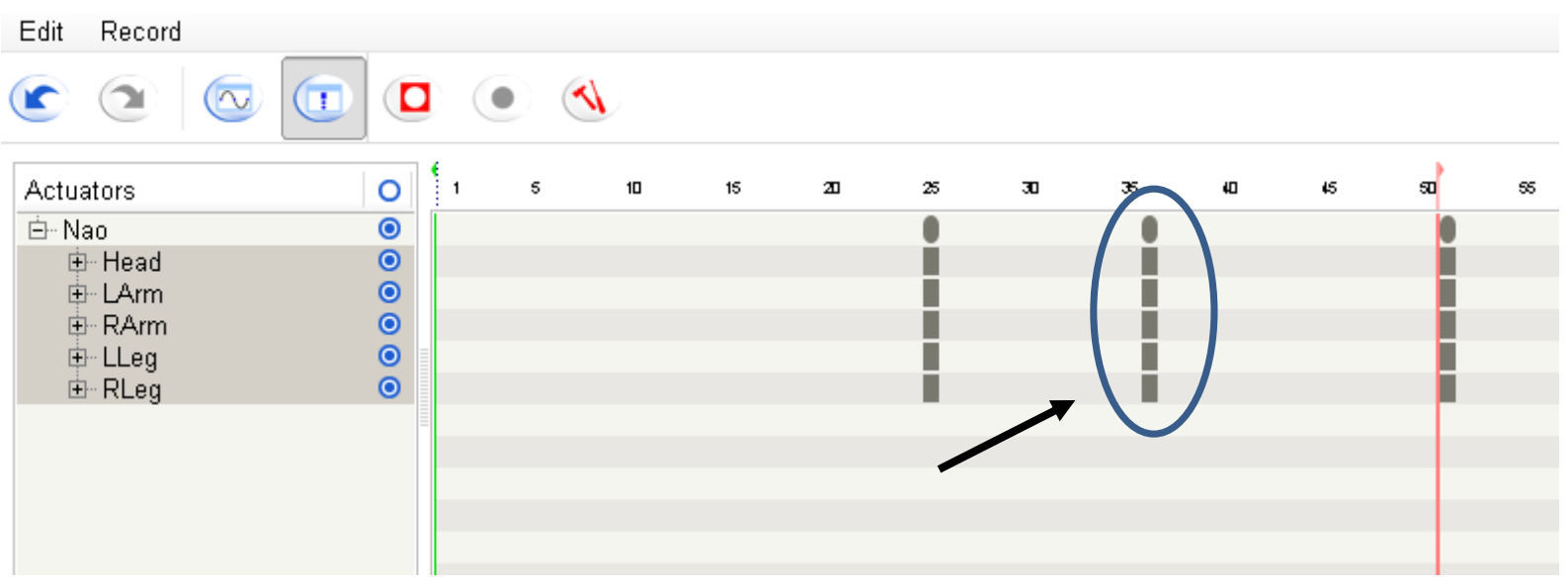

Für die Vorgehensweise, die Bewegungen flüssig aussehen $\mathrm{zu}$ lassen und stimmig aufeinander abzustimmen, wird an dieser Stelle auf die Dokumentation ${ }^{30}$ von Aldebaran Robotics verwiesen. Auf der beiliegenden DVD befindet sich des Weiteren eine ausführliche Ausarbeitung von Valentin Bertrand ${ }^{31}$ zur Benutzung des Animationsmodus. Der Fokus dieses Tutorials liegt in der Exportfunktion in C++.

\footnotetext{
${ }^{30} \mathrm{https}: / /$ community.aldebaran.com/doc/1-14/software/choregraphe/animation_mode.html, Zugriff 28.07.14

${ }^{31}$ Animateur bei Aldebaran Robotics
} 
A.1. Erstellung von Motions mittels des Animationsmodus und Export in $\mathrm{C}++$

Nachdem Sie das komplette Bewegungsmuster erstellt haben, wählen Sie im Timeline-Editor Edit $\rightarrow$ Export motion to clipboard $\rightarrow \mathrm{C}++$ und wählen Sie beizer oder simplified aus.

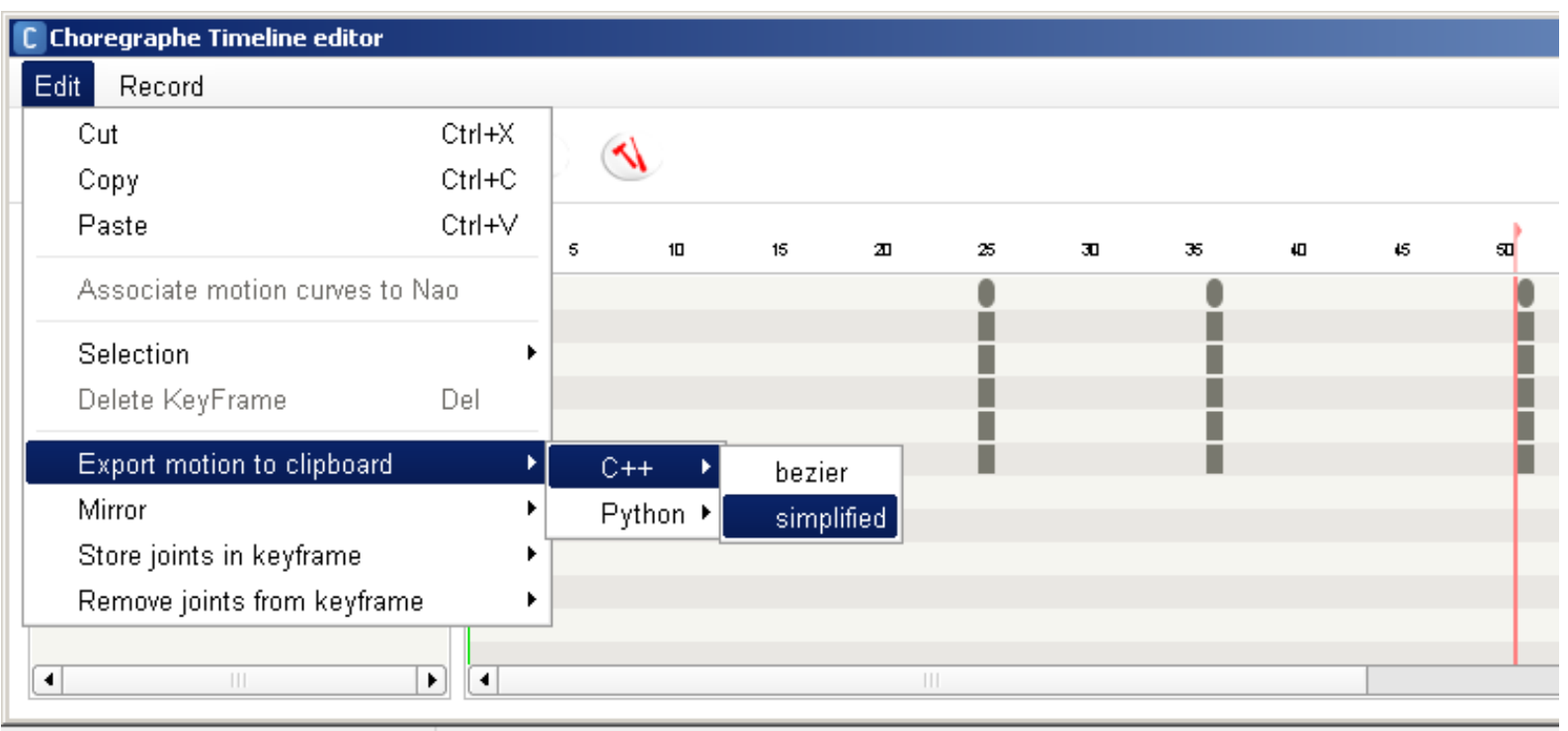

In der Zwischenablage befindet sich nun die Motion im C++-Code. Wechseln Sie in ihre Entwicklungsumgebung und fügen Sie den Inhalt an die entsprechende Stelle im Quellcode ein.

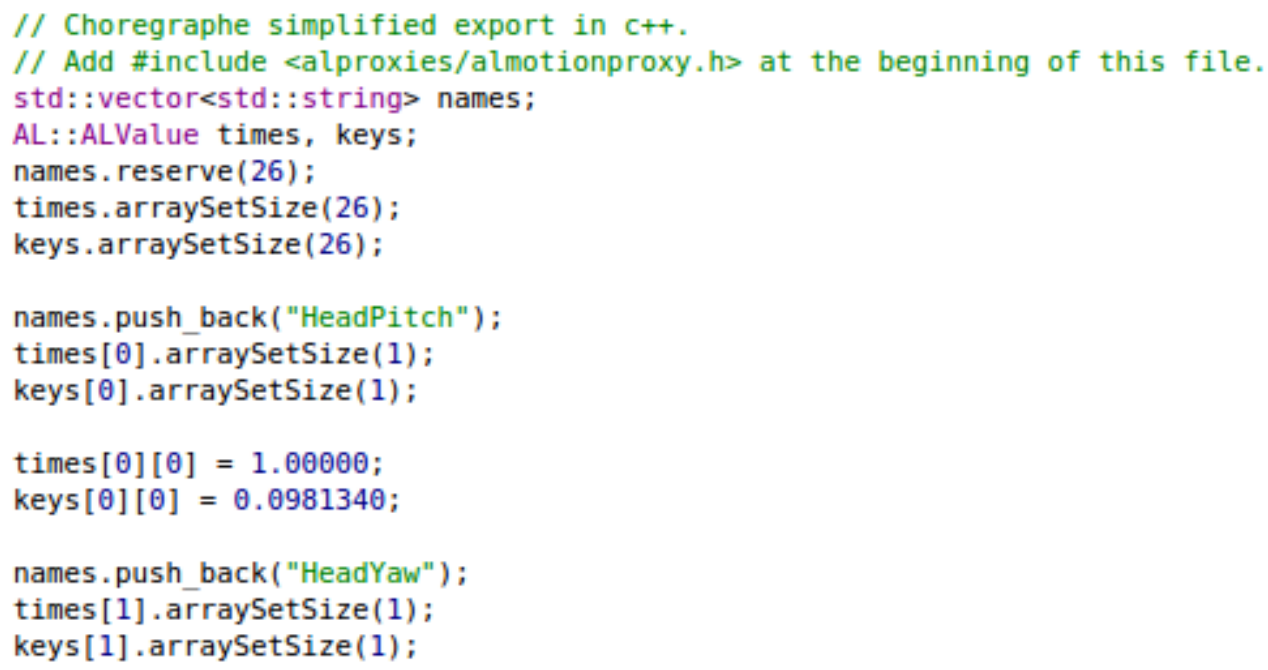

(Quellcode gekürzt) 


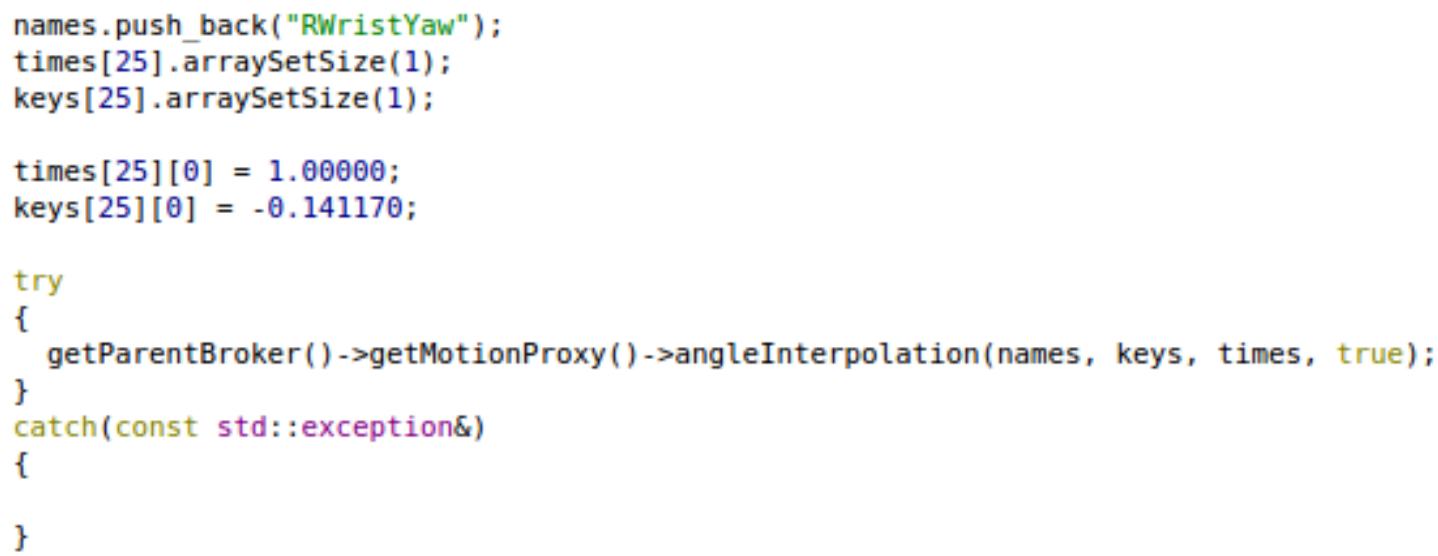

Die obige Abbildung enthält den simplifizierten exportierten Quellcode und ist vom Autor gekürzt. Choregraphe erstellt einen String-Vektor names und zwei ALValue-Werte times und keys. Die einzelnen Motorengelenke „HeadYaw“, „RWristYaw“ etc. werden in der richtigen Reihenfolge dem Vektor hinzugefügt. In der try-catch-Anweisung werden die Parameter names, keys und times der Methode angleInterpolation() übergeben. Der letzte Parameter gibt an, ob die Winkelwerte absolut oder relativ angegeben werden. Diese Methode, welche vom NAOqi-eigenen Modul ALMotion bereitgestellt wird, führt die Bewegung aus.

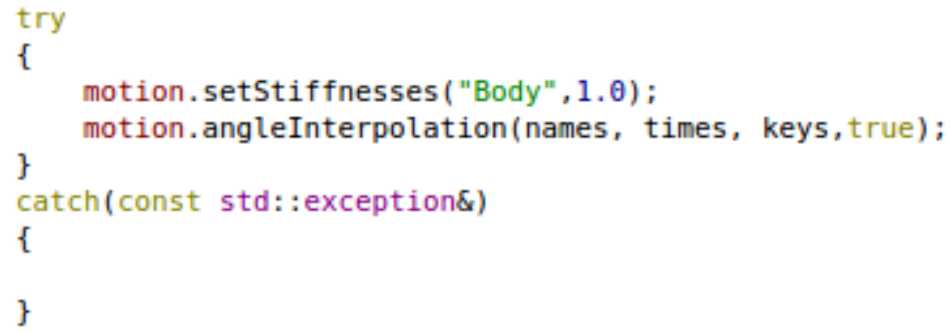

Der Aufruf der Methode sollte über das vorher angelegte Motion-Objekt erfolgen. $\mathrm{Zu}$ beachten ist, dass die stiffness vor Ausführen der angleInterpolation()-Methode eingeschaltet wird, da sich der Roboter sonst nicht bewegt. 


\section{A.2. Programmablaufplan Modul „Moderator“}

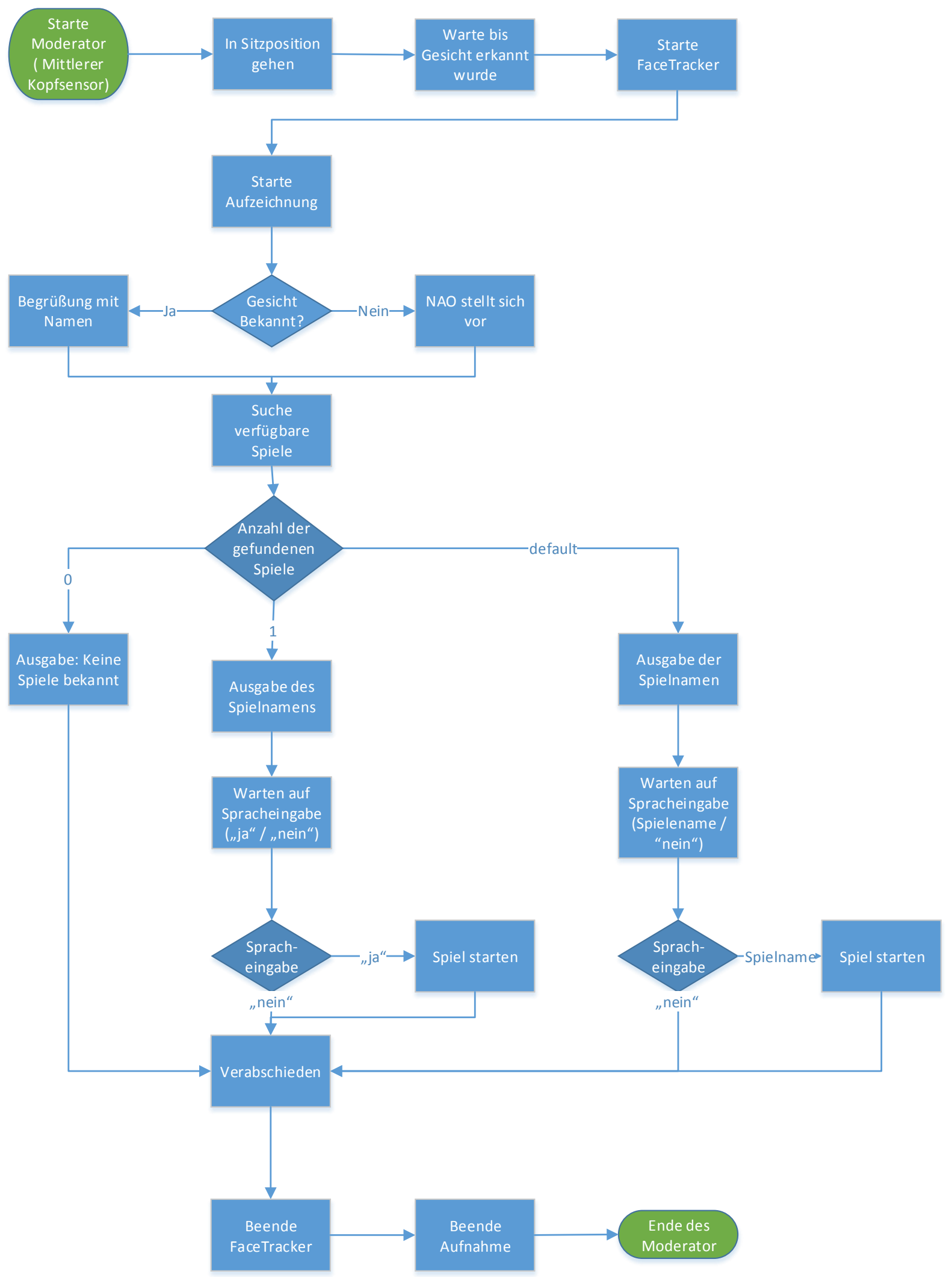




\begin{tabular}{|l|l|l|}
\hline Bewertungsbogen für Theo (9 Jahre) & Versuch: & 1 \\
\hline Eine erste Interaktion mit dem Roboter NAO & \\
\hline Erklärung der Bewertung & \\
\hline keine Reaktion & Das Kind steht herum und zeigt keine Reaktion. \\
\hline neutral & Das Kind reagiert erst nach mehrmaligem Auffordern des Therapeuten. \\
\hline wenig interessiert & Das Kind benötigt maximal 2-3 Aufforderungen um mit dem Roboter zu sprechen/agieren. \\
\hline interessiert & Das Kind spicht/agiert sofort nach Aufforderung des Therapeuten mit dem Roboter. \\
\hline sehr interessiert & Das reagiert sofort auf den Roboter ohne Aufforderung. \\
\hline
\end{tabular}

\begin{tabular}{|c|c|c|c|c|c|c|}
\hline $\begin{array}{c}\text { Wertung } \\
\text { Funktion des NAO }\end{array}$ & \begin{tabular}{|l|} 
keine \\
Reaktion - \\
\end{tabular} & neutral & $\begin{array}{l}\text { wenig } \\
\text { interessiert }=\end{array}$ & interessiert & $\begin{array}{l}\text { sehr } \\
\text { interessiert - }\end{array}$ & wiederholungen \\
\hline Präsens im Raum (NAO sitzt) & & & & & $x$ & - \\
\hline Bewegt Kopf nach rechts und links & & & $x$ & & & 2 \\
\hline steht aus der Sitzposition auf & & & & & $x$ & 3 \\
\hline spielt eine Musikdatei ab & $x$ & & & & & 1 \\
\hline winkt im Stehen & & & & $x$ & & 1 \\
\hline NAO fordert auf den Fuß zu berühren & & & & & $x$ & $>10$ \\
\hline
\end{tabular}

Für die Auswertung reicht es ein $\mathrm{X}$ in das jeweilige Feld zu setzen.

Die Anzahl der Wiederholungen werden als Zahl eingetragen. 


\section{Bewertungsbogen für Theo (9 Jahre)}

\begin{tabular}{|l|l|l|l|}
\hline & Versuch: & 2 \\
\hline & & & \\
\hline & & & \\
\hline
\end{tabular}

Eine zweite Interaktion mit dem Roboter NAO

Erklärung der Bewertung

\begin{tabular}{|c|c|}
\hline keine Reaktion & Das Kind steht herum und zeigt keine Reaktion. \\
\hline neutral & Das Kind reagiert erst nach mehrmaligem Auffordern des Therapeuten. \\
\hline wenig interessiert & Das Kind benötigt maximal 2-3 Aufforderungen um mit dem Roboter zu sprechen/agieren. \\
\hline interessiert & Das Kind spicht/agiert sofort nach Aufforderung des Therapeuten mit dem Roboter. \\
\hline sehr interessiert & Das reagiert sofort auf den Roboter ohne Aufforderung. \\
\hline
\end{tabular}

\begin{tabular}{|c|c|c|c|c|c|c|}
\hline $\begin{array}{c}\text { Wertung } \\
\text { Funktion des NAO } \\
\end{array}$ & \begin{tabular}{|l|} 
keine \\
Reaktion \\
\end{tabular} & neutral & $\begin{array}{l}\text { wenig } \\
\text { interessiert }\end{array}$ & interessiert & $\begin{array}{l}\text { sehr } \\
\text { interessiert }\end{array}$ & wiederholungen \\
\hline Präsens im Raum (NAO sitzt) & & & & & $\mathrm{x}$ & - \\
\hline Winken & & & & $x$ & & 2 \\
\hline Regeln erklären (deutsch) & & $x$ & & & & 3 \\
\hline Therapeut wiederholt Aufforderung & & & & $x$ & & $>10$ \\
\hline spielt eine Musikdatei ab & & & & $x$ & & 2 \\
\hline- & & & & & & - \\
\hline tanzt Tai Chi & & & & & $x$ & 2 \\
\hline
\end{tabular}

Für die Auswertung reicht es ein $\mathrm{X}$ in das jeweilige Feld zu setzen.

Die Anzahl der Wiederholungen werden als Zahl eingetragen.

* der Roboter hat die Aufforderung nur verbal geäußert 


\begin{tabular}{|c|c|c|c|c|}
\hline \multicolumn{2}{|c|}{ Bewertungsbogen für Theo (9 Jahre) } & Versuch: & 3 & 03.07 .2014 \\
\hline \multicolumn{5}{|l|}{ Eine dritte Interaktion mit dem Roboter NAO } \\
\hline \multicolumn{5}{|l|}{\begin{tabular}{|l|l|l|l|l} 
Erklärung der Bewertung \\
\end{tabular}} \\
\hline keine Reaktion & \multicolumn{4}{|c|}{ Das Kind steht herum und zeigt keine Reaktion. } \\
\hline neutral & \multicolumn{4}{|c|}{ Das Kind reagiert erst nach mehrmaligem Auffordern des Therapeuten. } \\
\hline wenig interessiert & \multicolumn{4}{|c|}{ Das Kind benötigt maximal 2-3 Aufforderungen um mit dem Roboter zu sprechen/agieren. } \\
\hline sehr interessiert & \multicolumn{4}{|c|}{ Das reagiert sofort auf den Roboter ohne Aufforderung. } \\
\hline
\end{tabular}

\begin{tabular}{|c|c|c|c|c|c|c|}
\hline $\begin{array}{c}\text { Wertung } \\
\text { Funktion des NAO }\end{array}$ & $\begin{array}{l}\text { keine } \\
\text { Reaktion - }\end{array}$ & neutral - & $\begin{array}{l}\text { wenig } \\
\text { interessiert }\end{array}$ & interessiert & $\begin{array}{l}\text { sehr } \\
\text { interessiert }\end{array}$ & wiederholungen \\
\hline Begrüßung & & & $x$ & & & 2 \\
\hline Winken & & $x$ & & & & 2 \\
\hline Regeln erklären (deutsch) & & $x$ & & & & 1 \\
\hline spielt eine Musikdatei ab & & & & $x$ & & 11 \\
\hline spricht englisch & & & $x$ & & & 10 \\
\hline tanzt Tai Chi & & & & $x$ & & 1 \\
\hline
\end{tabular}

Für die Auswertung reicht es ein $X$ in das jeweilige Feld zu setzen.

Die Anzahl der Wiederholungen werden als Zahl eingetragen.

* der Roboter hat die Aufforderung verbal geäußert und das zu berührende Körperteil bewegt

Die Sätze wurden gekürzt und vereinfacht. Gleichzeitig zur Aufforderung wird eine Bewegung ausgeführt Es wurde einmal des englische und einmal das deutsche Modul abgespielt 


\section{Bewertungsbogen für Theo (9 Jahre)}

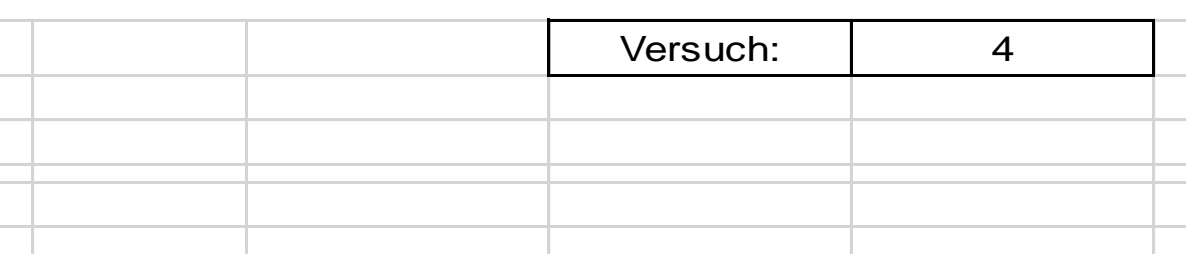

Eine dritte Interaktion mit dem Roboter NAO

Erklärung der Bewertung

\begin{tabular}{|l|l|}
\hline keine Reaktion & Das Kind steht herum und zeigt keine Reaktion. \\
\hline neutral & Das Kind reagiert erst nach mehrmaligem Auffordern des Therapeuten. \\
\hline wenig interessiert & Das Kind benötigt maximal 2-3 Aufforderungen um mit dem Roboter zu sprechen/agieren. \\
\hline interessiert & Das Kind spicht/agiert sofort nach Aufforderung des Therapeuten mit dem Roboter. \\
\hline sehr interessiert & Das reagiert sofort auf den Roboter ohne Aufforderung. \\
\hline
\end{tabular}

\begin{tabular}{|c|c|c|c|c|c|c|}
\hline $\begin{array}{c}\text { Wertung } \\
\text { Funktion des NAO } \\
\end{array}$ & \begin{tabular}{|l|} 
keine \\
Reaktion
\end{tabular} & neutral & $\begin{array}{l}\text { wenig } \\
\text { interessiert - }\end{array}$ & interessiert & $\begin{array}{l}\text { sehr } \\
\text { interessiert }\end{array}$ & wiederholungen \\
\hline Präsens im Raum (NAO sitzt) & & & & & $x$ & - \\
\hline Winken & & & & $x$ & & 2 \\
\hline Regeln erklären (deutsch) & & $x$ & & & & 1 \\
\hline Therapeut wiederholt Aufforderung & & & & & $x$ & 5 \\
\hline spielt eine Musikdatei ab & & & & $x$ & & 8 \\
\hline spricht englisch & & & $x$ & & & 10 \\
\hline tanzt Tai Chi & & & & & $x$ & 1 \\
\hline
\end{tabular}

Für die Auswertung reicht es ein $\mathrm{X}$ in das jeweilige Feld zu setzen.

Die Anzahl der Wiederholungen werden als Zahl eingetragen.

* der Roboter hat die Aufforderung verbal geäußert und das zu berührende Körperteil bewegt 


\section{A.7. Anwenderdokumentation}

Die folgenden Schritte erklären, wie die entwickelten Module auf dem NAO installiert und aktiviert werden können. Die folgende Vorgehensweise gilt für alle fünf Module, inklusive des Moderator Moduls.

1. Sie finden die jeweiligen fertigen Module als *.so- Dateien auf der DVD-Beilage Der Verzeichnispfad lautet:

$\backslash$ Quellcode \THW_Game_<Modulname>\build-cross-atom\sdk\lib\naoqi

2. Kopieren Sie die Datei in ein Verzeichnis ihrer Wahl.

3. Stellen Sie sicher, dass Sie mit Ihrem PC und dem NAO im selben WLAN sind.

4. Öffnen Sie unter Linux in der VM ein Terminal und navigieren Sie in das Verzeichnis, in dem Ihre *.so-Datei liegt, oder benutzen Sie ein FTP-Programm ${ }^{32}$ unter Windows.

5. Um nun eine .so-Datei auf den NAO zu senden, benutzen Sie folgenden Befehl: scp modulname.so nao@192.168.0.XXX:/

Geben Sie ein Passwort ein, wenn Sie dazu aufgefordert werden (default PW: nao).

6. Es wird empfohlen nun ein zweites Terminal zu öffnen.

7. Wechseln Sie in das neue Terminal und verbinden Sie sich mit dem NAO über folgenden Befehl:

ssh nao@192.168.0.XXX

Geben Sie wieder das eben verwendete Passwort ein, wenn Sie dazu aufgefordert werden.

8. Geben sie Is ein und überprüfen Sie, ob die <modulname>.so-Datei erfolgreich hochgeladen wurde.

9. Nun müssen Sie in der autoload. ini das Modul eintragen, damit es beim Starten der Naoqi geladen wird, öffnen Sie dazu mit dem Befehl:

vi /naoqi/preferences/autoload.ini die autoload.ini

\footnotetext{
${ }^{32}$ http://winscp.net/download/winscp554setup.exe Zugriff: 01.08.2014
} 
10. Tragen Sie unter [user] den Pfad zu allen <Modulname>.so-Dateien ein, wie in nachfolgender Abbildung zu sehen ist.

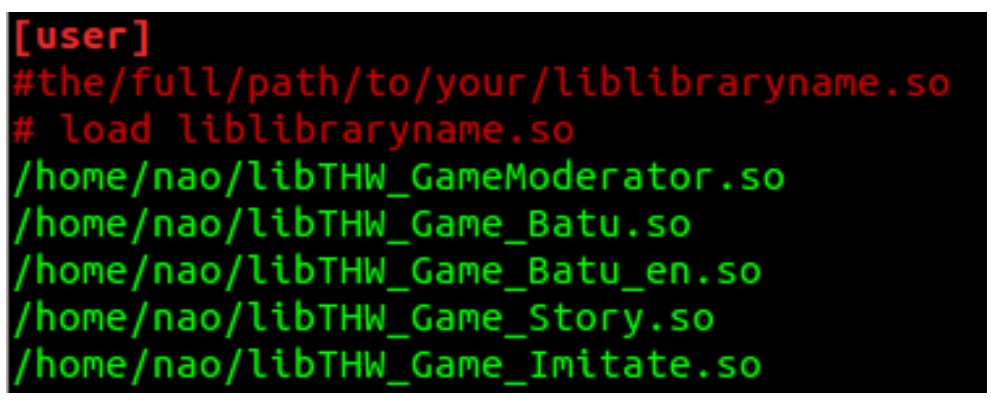

12. Um das Modul nun zu laden, muss abschließend die NAOqi neu gestartet werden.

Öffnen Sie dazu einen Browser und geben folgenden Pfad in die Adresszeile ein:

\subsubsection{XXX/\#naoqi}

und klicken Sie auf den Button Restart

\section{NaoQi is currently running.

Start Stop Restart

13. Die Module sollten nach dem Neustart und dem Drücken von F5 in der Liste zu sehen sein.

ALTextToSpeech
ALVideoDevice
ALVideoRecorder
ALVisionRecognition
ALVisionToolbox
ALVisualCompass
DCM
THWildGameModerator
THWild_Game__
THWild_Game__-_
THWild_Game_Imitate
THWild_Game_Story

14. Nun kann über den mittleren Kopfsensor das Moderator-Modul gestartet werden und auf alle implementierten Module zugegriffen werden.

Hinweis:

Nachdem Sie das Moderator-Modul über den Kopfsensor gestartet haben, müssen Sie dem NAO in die Augen schauen, damit er Ihr Gesicht erkennt und mit der Begrüßung beginnt. 


\section{A.8. Entwicklerdokumentation}

Die folgende Anleitung beschreibt die Integration des Quellcodes mittels qibuild in ein neues Projekt, für die Weiterentwicklung und Anpassung der Module im QTCreator.

1. Öffnen Sie ein Terminal in der VM und geben Sie folgende Befehle nach einander ein. $<$ Modulname $>$ ist hierbei immer durch den jeweiligen Modulnamen zu ersetzten.

2. Workspace anlegen (dieser Schritt muss nur angelegt werden, wenn noch kein Ordner mit einer cross-atom tool chain erstellt wurde)

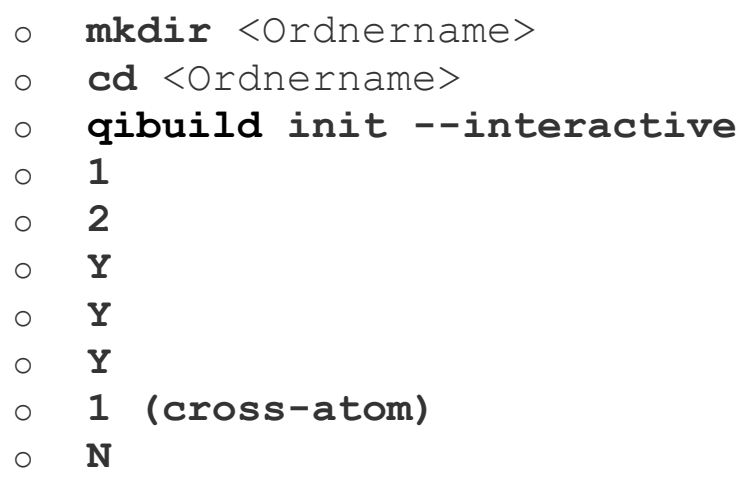

Folgende Schritte müssen für jedes Modul ausgeführt werden um es im QtCreator öffnen und editieren zu können:

3. $\quad$ cd $<$ Ordnername $>$ (workspace mit cross-atom toolchain)

4. erstellen Sie einen Ordner der genauso heißt wie der Projektordner auf der DVD mit folgendem Befehl:

qibuild create <THW_Game_<Modulname>

7. wechseln Sie in das angelegte Verzeichnis mit cd <THW_Game_<Modulname>

6. kopieren Sie in das eben angelegte Verzeichnis alle Dateien aus dem Projektordner auf der DVD mit $\mathrm{cp} / \mathrm{pfad} / \mathrm{zum} /$ Projektorder/auf/der/dvd/* . * .

7. rm -r CMakeText.user.txt build-cross-atom

8. qibuild configure

$\circ \mathrm{Y}$ 
9. Um das Projekt im QtCreator zu öffnen geben Sie folgende Zeile ein

\section{qibuild open}

\section{(1) CMake Wizard}

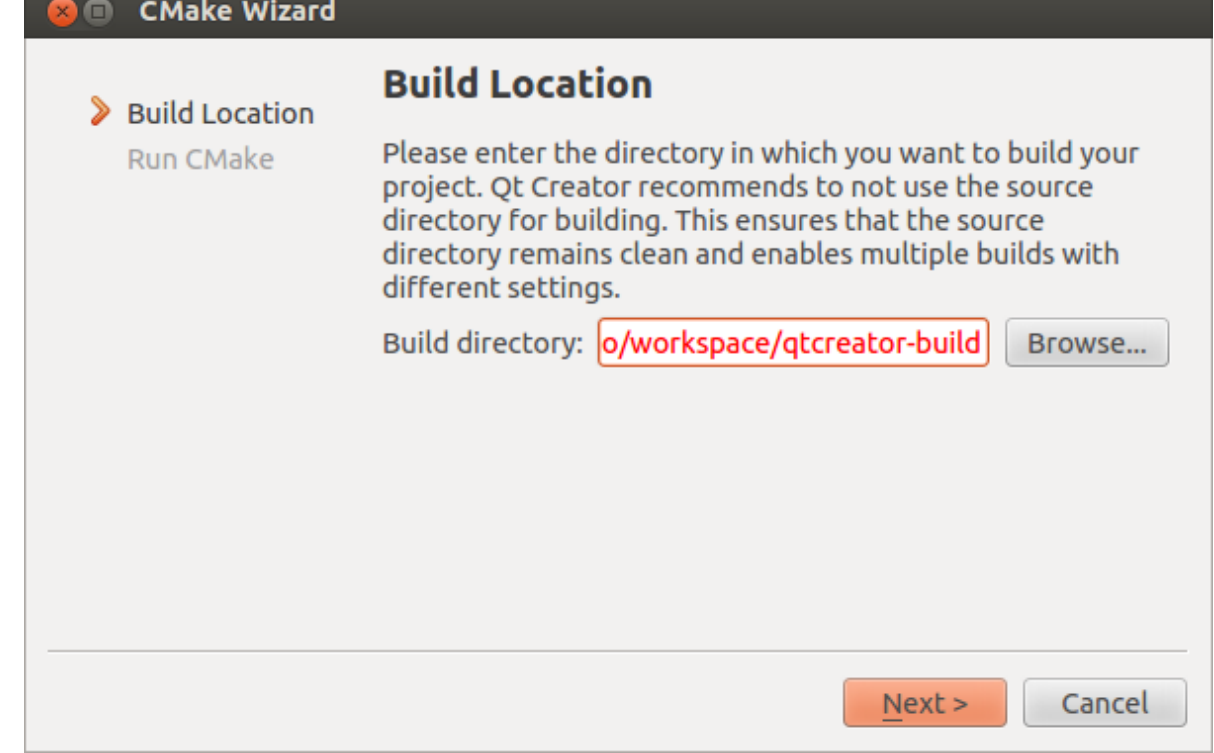

Abbildung 69 - CMake Wizard

10. Wählen Sie das Verzeichnis

/home/nao/workspace/THW_Game_<Modulname>/build-cross-atom als build directory aus und klicken sie auf open

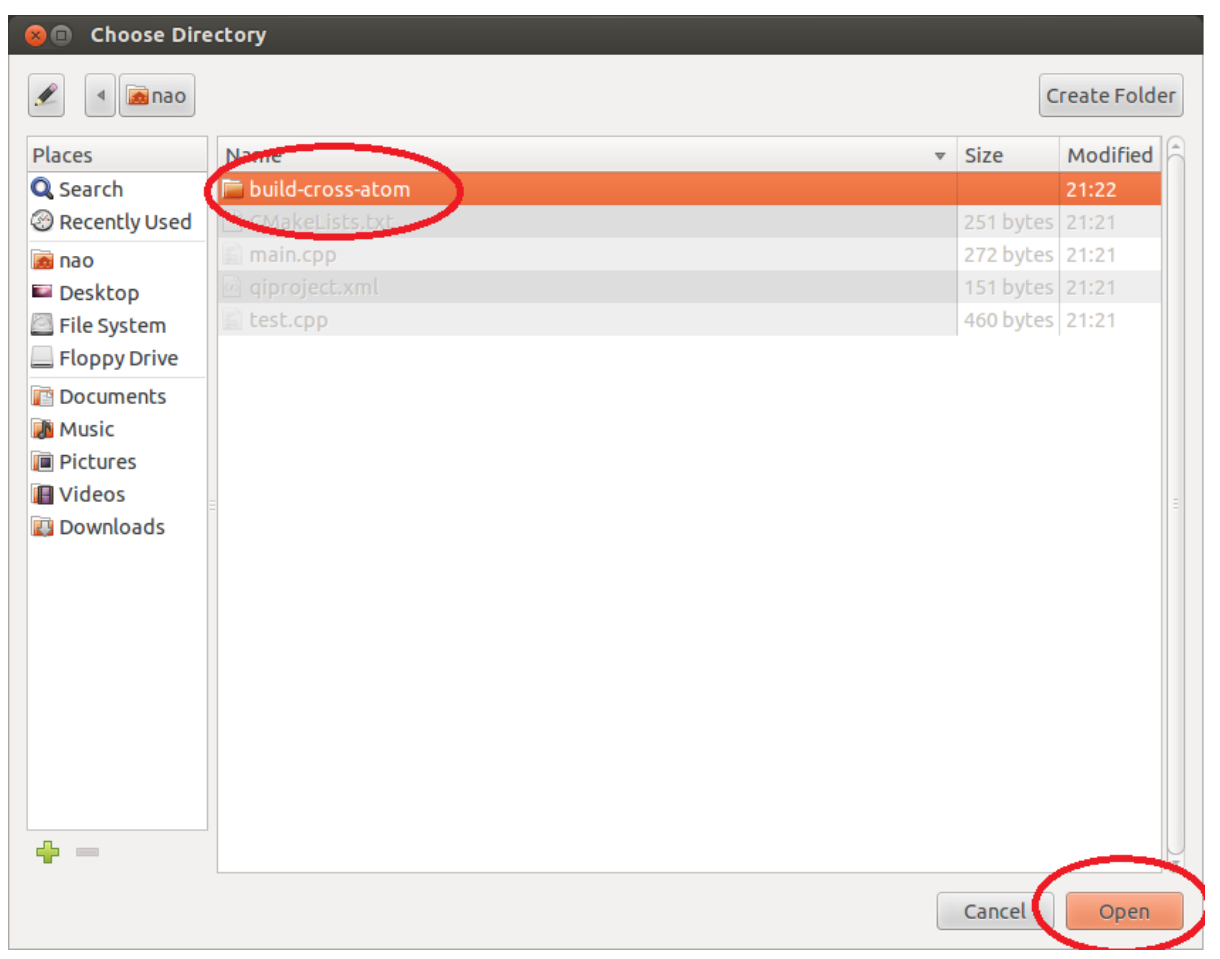

Abbildung 70 - Auswahl des build-cross-atom Ordners 
11. klicken Sie auf Next

12. klicken Sie run CMake

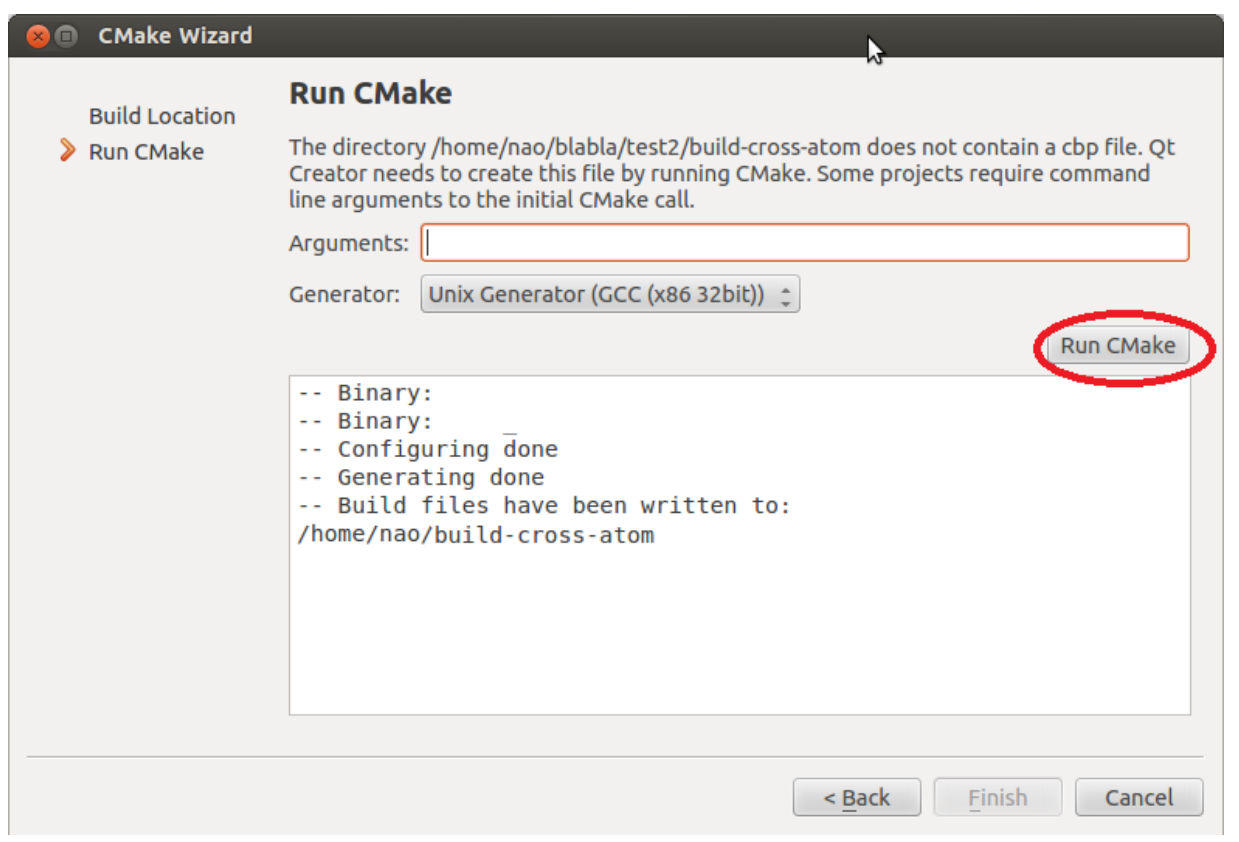

Abbildung 71 - CMake Wizard - Run CMake um das Projekt zu initialisieren

13. klicken Sie dann auf Finish

14. Um eine .so-Datei zu erstellen klicken Sie auf das Hammersymbol. Die erstellte *.so-Datei befindet sich dann im Verzeichnis

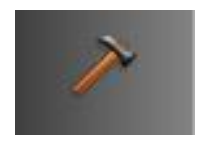

<THW_Game_<Modulname>/build-cross-atom/sdk/lib/naoqi/

Um die *.so Datei auf den NAO zu bringen, folgen Sie den Anweisungen in der Anwenderdokumentation.

Die jeweilige HTML-Dokumentation befindet sich auf der DVD unter folgendem Pfad:

\section{/THW_Game_<Modulname>/documentation/index.html}

An dieser Stelle wird auf die ausführliche Doxygen-Dokumentation, die auf der DVDBeigabe zu finden ist, verwiesen. Sie enthält für jedes entwickelte Modul eine übersichtliche HTML basierte Dokumentation inklusive visualisierter Methodenaufrufe und Klassendiagramme. Des Weiteren werden die Abhängigkeiten und der Gesamtüberblick der Module grafisch dargestellt. Um einen kurzen Überblick über alle Methoden zu verschaffen, werden nachfolgend die Abhängigkeiten zwischen den Methodenaufrufen jedes einzelnen Moduls unkommentiert dargestellt. In der Dokumentation werden die Grafiken ausführlich und nachvollziehbar beschrieben. 
Modul „Moderator“

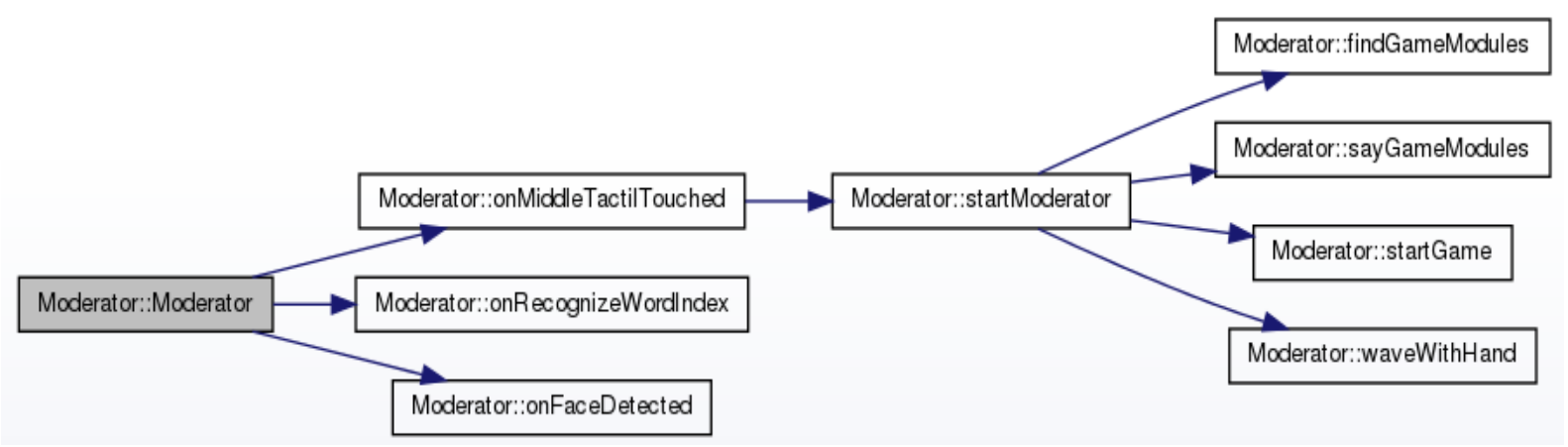

Abbildung 72 - Methodenübersicht und Aufrufabhängigkeiten des Moderator Moduls

Modul „Gefühle“

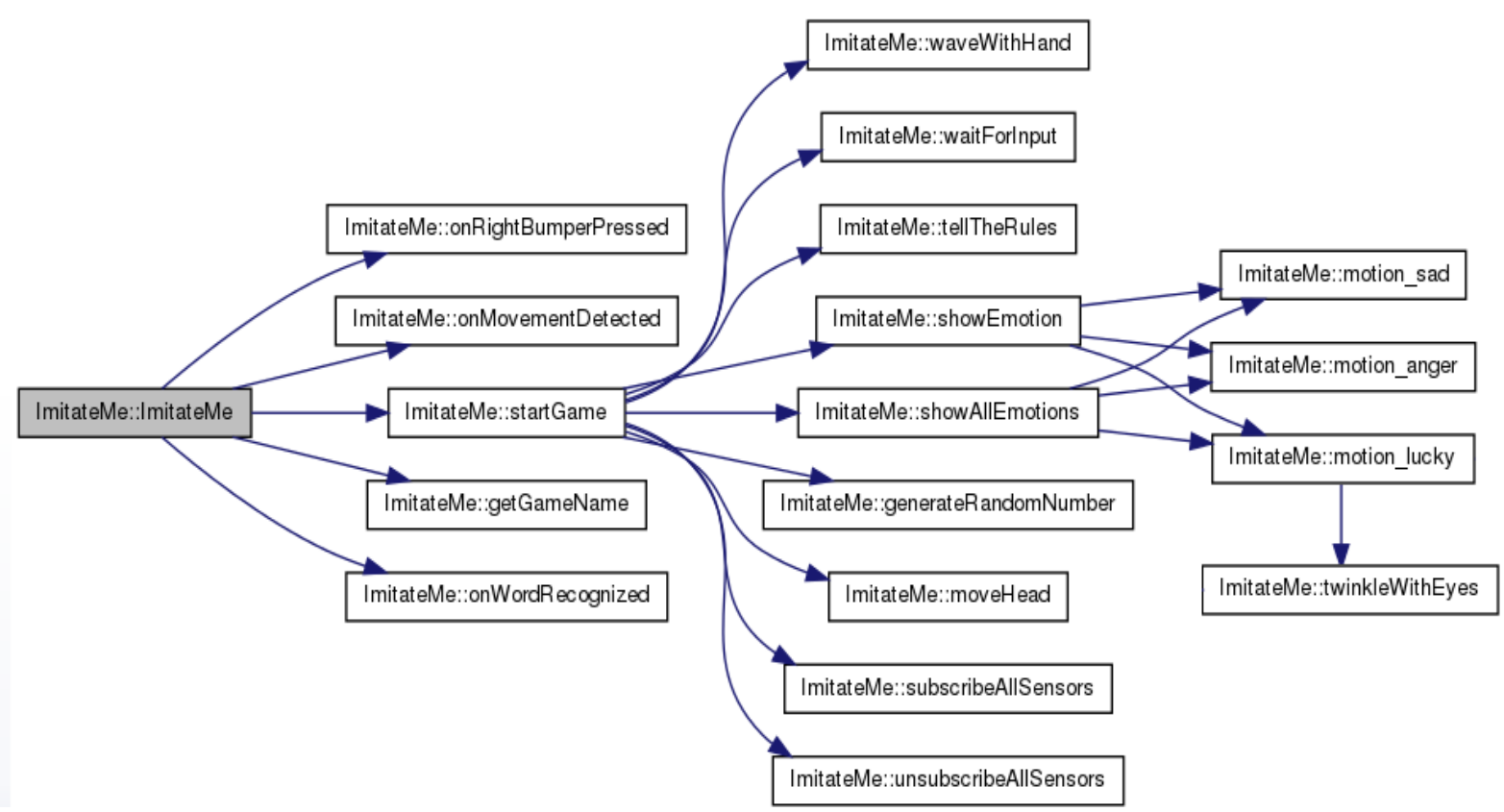

Abbildung 73 - Methodenübersicht und Aufrufabhängigkeiten des Moduls „Gefühle“ 
Modul „Berühre Mich“ (,Touch Me“)

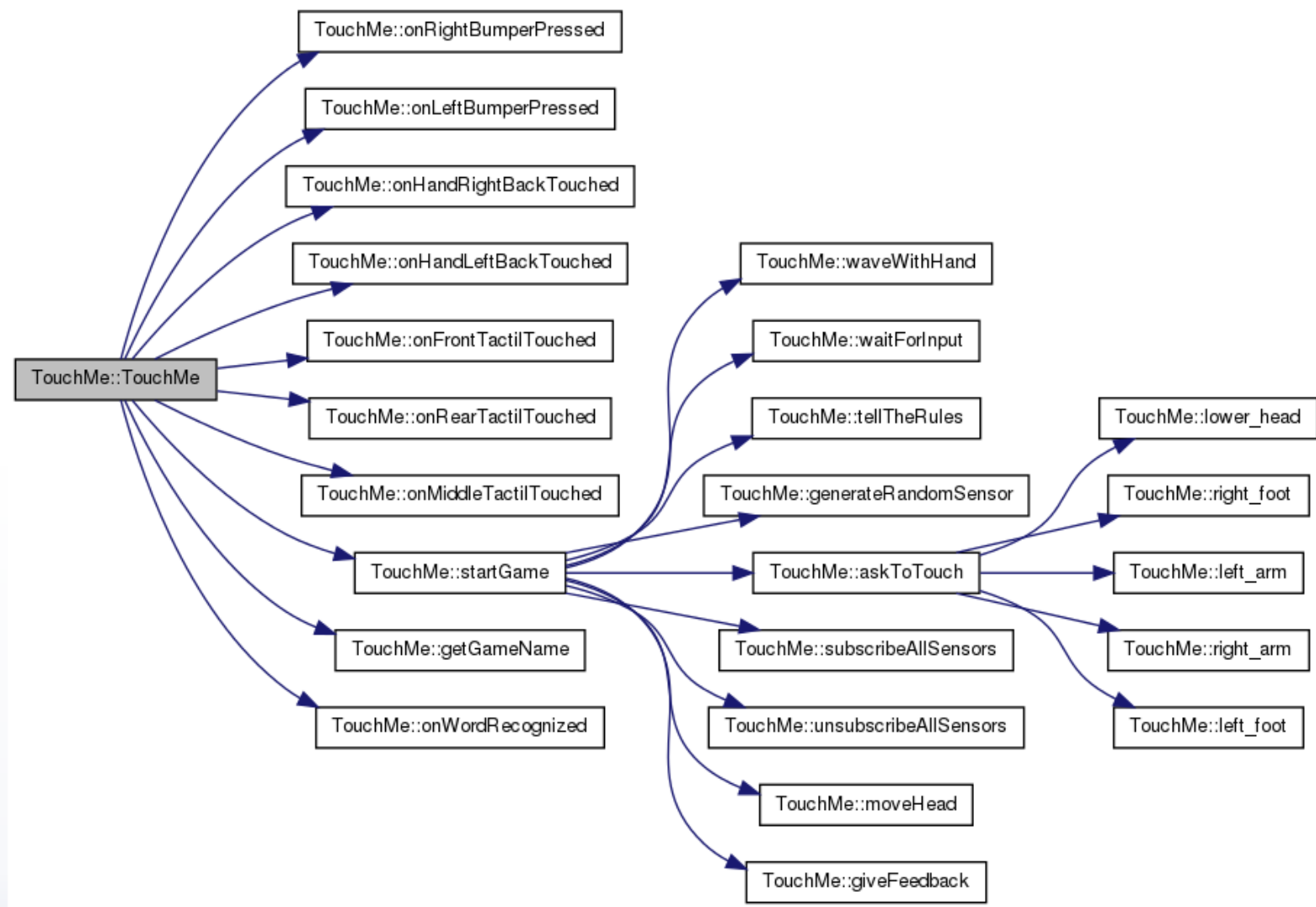

Abbildung 74 - Methodenübersicht und Aufrufabhängigkeiten der Module „Berühre Mich“ und „Touch Me“

Modul „Geschichte“

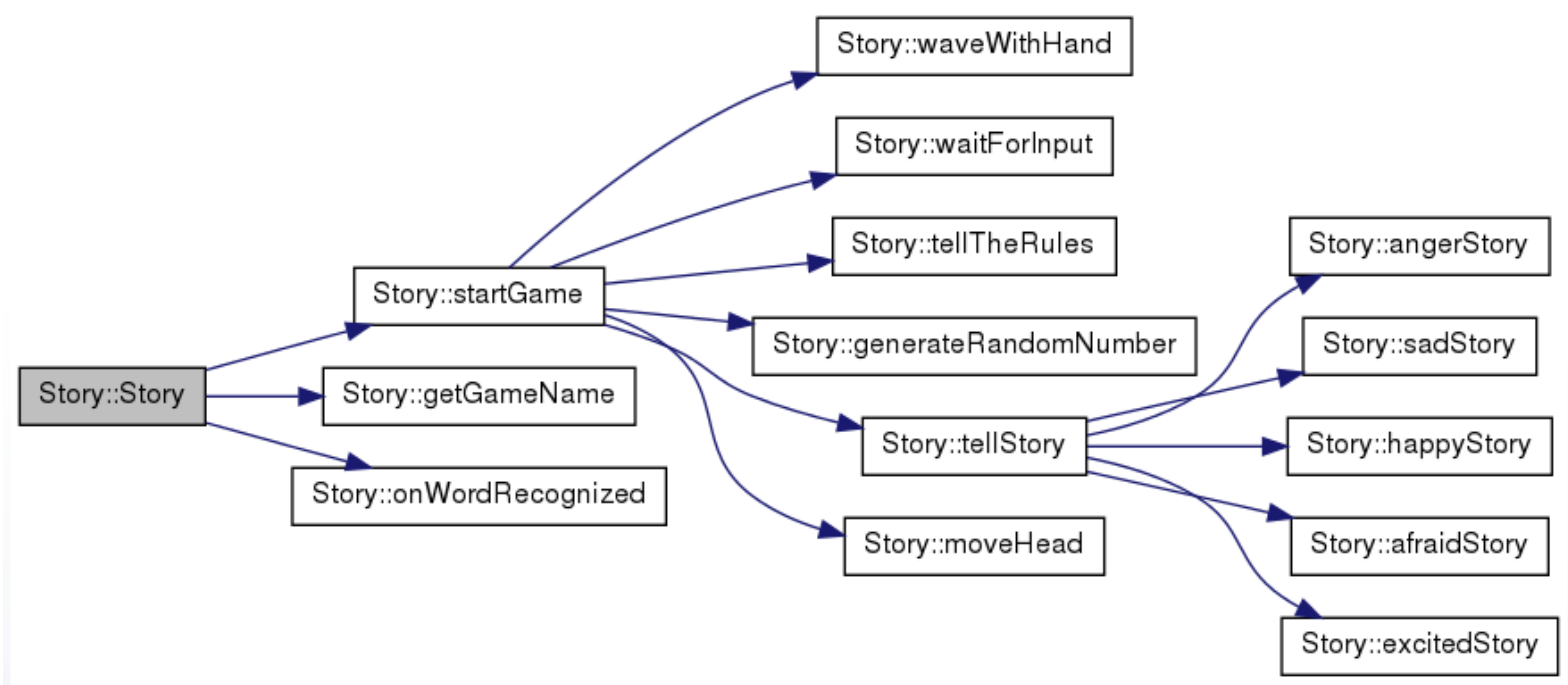

Abbildung 75 - Methodenübersicht und Aufrufabhängigkeiten des Moduls „Geschichte“ 


\section{B. Beilagen \\ B.1. DVD-Beigabe}

Die dieser Arbeit beigelegte DVD enthält folgende Daten:

\section{Projekte:}

Projektverzeichnisse aller fünf Module

- THW_Game_Batu

- THW_Game_Batu_en

- THW_Game_Imitate

- THW_Game_Story

- THW_Game_Moderator

inklusive Quellcode- und Doxygen-Dokumentation.

\section{Tutorial:}

$$
\begin{array}{ll}
\text { Robotic Animation - HowTo } & \text { Valentin Bertrand } \\
\text { Erstellung eigener Projekte } & \text { Georg Labbé }
\end{array}
$$

\section{Die Arbeit:}

Die Bachelorarbeit in digitaler Form im PDF-Format

\section{Anwenderdokumentation:}

Die Installations- und Benutzeranleitung für die Verwendung der Module auf einem neuen NAO. 